Supporting Information for:

\title{
Synthesis, Characterization, and Biological Activity of Water- Soluble, Dual Anionic and Cationic Ruthenium-Arene Complexes Bearing Imidazol(in)ium-2-dithiocarboxylate Ligands
}

Mohammed Zain Aldin, ${ }^{\dagger}$ Guillermo Zaragoza, ${ }^{*}$ William Deschamps,${ }^{\S}$ Jean-Claude Didelot Tomani,${ }^{\S}$ Jacob Souopgui, ${ }^{\S}$ and Lionel Delaude ${ }^{*}, \dagger$

${ }^{\dagger}$ Laboratory of Catalysis, MolSys Research Unit, Institut de Chimie Organique (B6a), Allée du six Août 13, Université de Liège, 4000 Liège, Belgium

Unidade de Difracción de Raios X, RIAIDT, Universidade de Santiago de Compostela, Campus Vida, 15782 Santiago de Compostela, Spain

$\S$ Department of Molecular Biology, Institute for Molecular Biology and Medicine, Université Libre de Bruxelles, 6041 Gosselies, Belgium

*E-mail:l.delaude@uliege.be 


\section{Table of Contents}

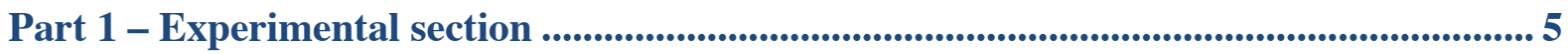

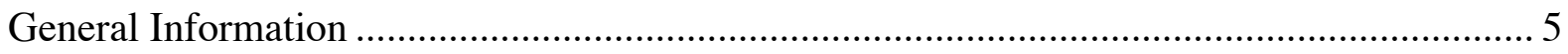

Synthesis of Monometallic Complexes 2a-e .............................................................. 5

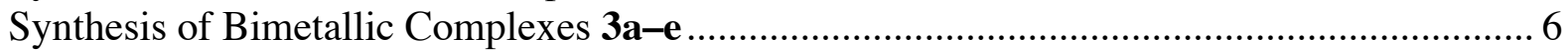

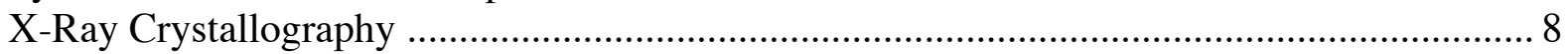

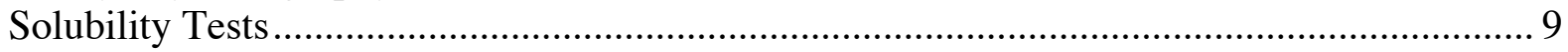

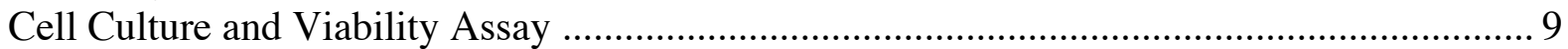

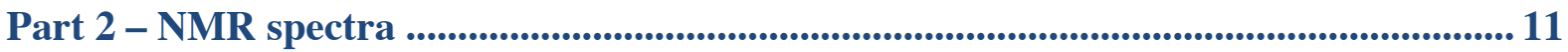

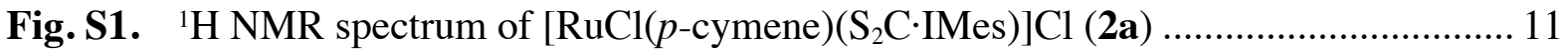

Fig. S2. ${ }^{13} \mathrm{C}\left\{{ }^{1} \mathrm{H}\right\}$ NMR spectrum of $\left[\mathrm{RuCl}(p\right.$-cymene $\left.)\left(\mathrm{S}_{2} \mathrm{C} \cdot \mathrm{IMes}\right)\right] \mathrm{Cl}(\mathbf{2 a}) \ldots \ldots \ldots \ldots \ldots \ldots \ldots . . . . . . . . . . . .12$

Fig. S3. ${ }^{13} \mathrm{C}\left\{{ }^{1} \mathrm{H}\right\}$ APT NMR spectrum of $\left[\mathrm{RuCl}(p\right.$-cymene $\left.)\left(\mathrm{S}_{2} \mathrm{C} \cdot \mathrm{IMes}\right)\right] \mathrm{Cl}(\mathbf{2 a}) \ldots \ldots \ldots \ldots . . . .12$

Fig. S4. ${ }^{13} \mathrm{C} C P D$ and APT NMR spectra of $\left[\mathrm{RuCl}(p\right.$-cymene $\left.)\left(\mathrm{S}_{2} \mathrm{C} \cdot \mathrm{IMes}\right)\right] \mathrm{Cl}(\mathbf{2 a}) \ldots \ldots \ldots . . .13$

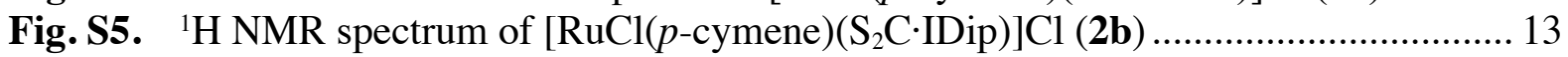

Fig. S6. ${ }^{13} \mathrm{C}\left\{{ }^{1} \mathrm{H}\right\}$ NMR spectrum of $\left[\mathrm{RuCl}(p\right.$-cymene $\left.)\left(\mathrm{S}_{2} \mathrm{C} \cdot \mathrm{IDip}\right)\right] \mathrm{Cl}(\mathbf{2 b}) \ldots \ldots \ldots \ldots \ldots \ldots \ldots \ldots . . . . . . . . . . . .14$

Fig. S7. ${ }^{13} \mathrm{C}\left\{{ }^{1} \mathrm{H}\right\}$ APT NMR spectrum of $\left[\mathrm{RuCl}(p\right.$-cymene $\left.)\left(\mathrm{S}_{2} \mathrm{C} \cdot \mathrm{IDip}\right)\right] \mathrm{Cl}(\mathbf{2 b}) \ldots \ldots \ldots \ldots \ldots . . .14$

Fig. S8. ${ }^{13} \mathrm{C} \mathrm{CPD}$ and APT NMR spectra of $\left[\mathrm{RuCl}(p\right.$-cymene $\left.)\left(\mathrm{S}_{2} \mathrm{C} \cdot \mathrm{IDip}\right)\right] \mathrm{Cl}(\mathbf{2 b}) \ldots \ldots \ldots . . . .15$

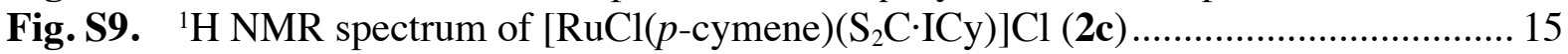

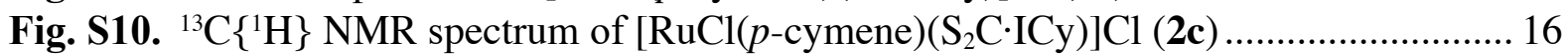

Fig. S11. ${ }^{13} \mathrm{C}\left\{{ }^{1} \mathrm{H}\right\}$ APT NMR spectrum of $\left[\mathrm{RuCl}(p\right.$-cymene $\left.)\left(\mathrm{S}_{2} \mathrm{C} \cdot \mathrm{ICy}\right)\right] \mathrm{Cl}(\mathbf{2 c}) \ldots \ldots \ldots \ldots \ldots \ldots . . . . . . . .16$

Fig. S12. ${ }^{13} \mathrm{C} \mathrm{CPD}$ and APT NMR spectra of $\left[\mathrm{RuCl}(p\right.$-cymene $\left.)\left(\mathrm{S}_{2} \mathrm{C} \cdot \mathrm{ICy}\right)\right] \mathrm{Cl}(\mathbf{2 c}) \ldots \ldots \ldots \ldots . . . . .17$

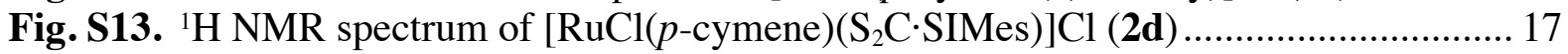

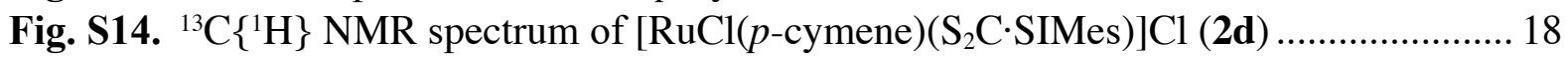

Fig. S15. ${ }^{13} \mathrm{C}\left\{{ }^{1} \mathrm{H}\right\}$ APT NMR spectrum of $\left[\mathrm{RuCl}(p\right.$-cymene $\left.)\left(\mathrm{S}_{2} \mathrm{C} \cdot \mathrm{SIMes}\right)\right] \mathrm{Cl}(\mathbf{2 d}) \ldots \ldots \ldots \ldots . . .18$

Fig. S16. ${ }^{13} \mathrm{C} \mathrm{CPD}$ and APT NMR spectra of $\left[\mathrm{RuCl}(p\right.$-cymene $\left.)\left(\mathrm{S}_{2} \mathrm{C} \cdot \mathrm{SIMes}\right)\right] \mathrm{Cl}(\mathbf{2 d}) \ldots \ldots . . . .19$

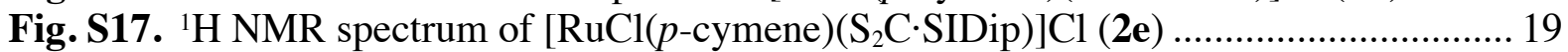

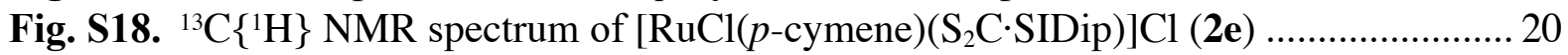

Fig. S19. ${ }^{13} \mathrm{C}\left\{{ }^{1} \mathrm{H}\right\}$ APT NMR spectrum of $\left[\mathrm{RuCl}(p\right.$-cymene $\left.)\left(\mathrm{S}_{2} \mathrm{C} \cdot \mathrm{SIDip}\right)\right] \mathrm{Cl}(\mathbf{2 e}) \ldots \ldots \ldots \ldots . . . . .20$

Fig. S20. ${ }^{13} \mathrm{C} C P D$ and APT NMR spectra of $\left[\operatorname{RuCl}(p\right.$-cymene $\left.)\left(\mathrm{S}_{2} \mathrm{C} \cdot \mathrm{SIDip}\right)\right] \mathrm{Cl}(\mathbf{2 e}) \ldots \ldots \ldots . .21$

Fig. S21. ${ }^{1} \mathrm{H}$ NMR spectrum of $\left[\mathrm{RuCl}(p\right.$-cymene $\left.)\left(\mathrm{S}_{2} \mathrm{C} \cdot \mathrm{IMes}\right)\right]\left[\mathrm{RuCl}_{3}(p\right.$-cymene $\left.)\right](\mathbf{3 a}) \ldots \ldots . .21$

Fig. S22. ${ }^{13} \mathrm{C}\left\{{ }^{1} \mathrm{H}\right\} \mathrm{NMR}$ spectrum of $\left[\mathrm{RuCl}(p\right.$-cymene $\left.)\left(\mathrm{S}_{2} \mathrm{C} \cdot \mathrm{IMes}\right)\right]\left[\mathrm{RuCl}_{3}(p\right.$-cymene $\left.)\right]$ (3a)22

Fig. S23. ${ }^{13} \mathrm{C}\left\{{ }^{1} \mathrm{H}\right\}$ APT NMR spectrum of $\left[\mathrm{RuCl}(p\right.$-cymene $\left.)\left(\mathrm{S}_{2} \mathrm{C} \cdot \mathrm{IMes}\right)\right]\left[\mathrm{RuCl}_{3}(p\right.$-cymene $\left.)\right]$ (3a)

Fig. S24. ${ }^{13} \mathrm{C} \mathrm{CPD}$ and APT NMR spectra of $\left[\mathrm{RuCl}(p\right.$-cymene $\left.)\left(\mathrm{S}_{2} \mathrm{C} \cdot \mathrm{IMes}\right)\right]\left[\mathrm{RuCl}_{3}(\mathrm{p}\right.$-cym-

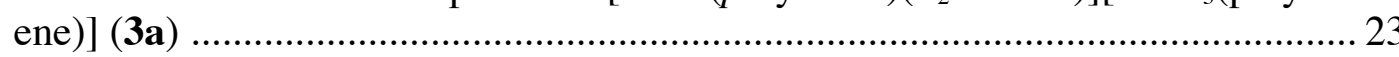

Fig. S25. ${ }^{1} \mathrm{H}$ NMR spectrum of $\left[\mathrm{RuCl}(p\right.$-cymene $\left.)\left(\mathrm{S}_{2} \mathrm{C} \cdot \mathrm{IDip}\right)\right]\left[\mathrm{RuCl}_{3}(p\right.$-cymene $\left.)\right](\mathbf{3 b}) \ldots \ldots . .23$

Fig. S26. ${ }^{13} \mathrm{C}\left\{{ }^{1} \mathrm{H}\right\} \mathrm{NMR}$ spectrum of $\left[\mathrm{RuCl}(p\right.$-cymene $\left.)\left(\mathrm{S}_{2} \mathrm{C} \cdot \mathrm{IDip}\right)\right]\left[\mathrm{RuCl}_{3}(p\right.$-cymene $\left.)\right](3 \mathbf{b}) 24$

Fig. S27. ${ }^{13} \mathrm{C}\left\{{ }^{1} \mathrm{H}\right\}$ APT NMR spectrum of $\left[\mathrm{RuCl}(p\right.$-cymene $\left.)\left(\mathrm{S}_{2} \mathrm{C} \cdot \mathrm{IDip}\right)\right]\left[\mathrm{RuCl}_{3}(p\right.$-cymene $\left.)\right]$ (3b)

Fig. S28. ${ }^{13} \mathrm{C} C P D$ and APT NMR spectra of $\left[\mathrm{RuCl}(p\right.$-cymene $\left.)\left(\mathrm{S}_{2} \mathrm{C} \cdot \mathrm{IDip}\right)\right]\left[\mathrm{RuCl}_{3}(p\right.$-cym-

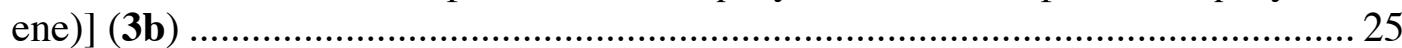

Fig. S29. ${ }^{1} \mathrm{H}$ NMR spectrum of $\left[\mathrm{RuCl}(p\right.$-cymene $\left.)\left(\mathrm{S}_{2} \mathrm{C} \cdot \mathrm{ICy}\right)\right]\left[\mathrm{RuCl}_{3}(p\right.$-cymene $\left.)\right](\mathbf{3 c}) \ldots \ldots . . .25$

Fig. S30. ${ }^{13} \mathrm{C}\left\{{ }^{1} \mathrm{H}\right\} \mathrm{NMR}$ spectrum of $\left[\mathrm{RuCl}(p\right.$-cymene $\left.)\left(\mathrm{S}_{2} \mathrm{C} \cdot \mathrm{ICy}\right)\right]\left[\mathrm{RuCl}_{3}(p\right.$-cymene $\left.)\right](3 \mathbf{c}) .26$

Fig. S31. ${ }^{13} \mathrm{C}\left\{{ }^{1} \mathrm{H}\right\}$ APT NMR spectrum of $\left[\mathrm{RuCl}(p\right.$-cymene $\left.)\left(\mathrm{S}_{2} \mathrm{C} \cdot \mathrm{ICy}\right)\right]\left[\mathrm{RuCl}_{3}(p\right.$-cymene $\left.)\right]$

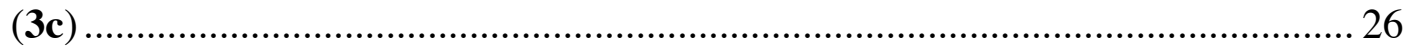

Fig. S32. ${ }^{13} \mathrm{C} \mathrm{CPD}$ and APT NMR spectra of $\left[\mathrm{RuCl}(p\right.$-cymene $\left.)\left(\mathrm{S}_{2} \mathrm{C} \cdot \mathrm{ICy}\right)\right]\left[\mathrm{RuCl}_{3}(p\right.$-cymene $\left.)\right]$ (3c)

Fig. S33. ${ }^{1} \mathrm{H}$ NMR spectrum of $\left[\mathrm{RuCl}(p\right.$-cymene $\left.)\left(\mathrm{S}_{2} \mathrm{C} \cdot \mathrm{SIMes}\right)\right]\left[\mathrm{RuCl}_{3}(p\right.$-cymene $\left.)\right](\mathbf{3 d}) \ldots . .27$ 
Fig. S34. ${ }^{13} \mathrm{C}\left\{{ }^{1} \mathrm{H}\right\}$ NMR spectrum of $\left[\operatorname{RuCl}(p\right.$-cymene $\left.)\left(\mathrm{S}_{2} \mathrm{C} \cdot \mathrm{SIMes}\right)\right]\left[\mathrm{RuCl}_{3}(p\right.$-cymene $\left.)\right](\mathbf{3 d})$

Fig. S35. ${ }^{13} \mathrm{C}\left\{{ }^{1} \mathrm{H}\right\}$ APT NMR spectrum of $\left[\operatorname{RuCl}(p\right.$-cymene $\left.)\left(\mathrm{S}_{2} \mathrm{C} \cdot \mathrm{SIMes}\right)\right]\left[\mathrm{RuCl}_{3}(p\right.$-cymene $\left.)\right]$ (3d) 28

Fig. S36. ${ }^{13} \mathrm{C} \mathrm{CPD}$ and APT NMR spectra of $\left[\mathrm{RuCl}(p\right.$-cymene $\left.)\left(\mathrm{S}_{2} \mathrm{C} \cdot \mathrm{SIMes}\right)\right]\left[\mathrm{RuCl}_{3}(p\right.$-cym-

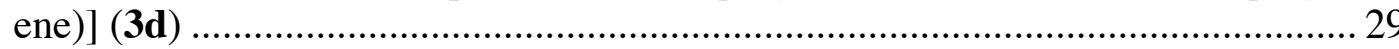

Fig. S37. ${ }^{1} \mathrm{H}$ NMR spectrum of $\left[\mathrm{RuCl}(p\right.$-cymene $\left.)\left(\mathrm{S}_{2} \mathrm{C} \cdot \mathrm{SIDip}\right)\right]\left[\mathrm{RuCl}_{3}(p\right.$-cymene $\left.)\right](\mathbf{3 e}) \ldots \ldots .29$

Fig. S38. ${ }^{13} \mathrm{C}\left\{{ }^{1} \mathrm{H}\right\}$ NMR spectrum of $\left[\mathrm{RuCl}(p\right.$-cymene $\left.)\left(\mathrm{S}_{2} \mathrm{C} \cdot \mathrm{SIDip}\right)\right]\left[\mathrm{RuCl}_{3}(p\right.$-cymene $\left.)\right](\mathbf{3 e})$

Fig. S39. ${ }^{13} \mathrm{C}\left\{{ }^{1} \mathrm{H}\right\}$ APT NMR spectrum of $\left[\operatorname{RuCl}(p\right.$-cymene $\left.)\left(\mathrm{S}_{2} \mathrm{C} \cdot \mathrm{SIDip}\right)\right]\left[\mathrm{RuCl}_{3}(p\right.$-cymene $\left.)\right]$ $(3 \mathrm{e})$

Fig. S40. ${ }^{13} \mathrm{C} C P D$ and APT NMR spectra of $\left[\mathrm{RuCl}(p\right.$-cymene $\left.)\left(\mathrm{S}_{2} \mathrm{C} \cdot \mathrm{SIDip}\right)\right]\left[\mathrm{RuCl}_{3}(p\right.$-cymene)] (3e)

Part 3 - IR spectra

Fig. S41. FT-IR spectrum of $\left[\mathrm{RuCl}(p\right.$-cymene $\left.)\left(\mathrm{S}_{2} \mathrm{C} \cdot \mathrm{IMes}\right)\right]\left[\mathrm{RuCl}_{3}(p\right.$-cymene $\left.)\right](\mathbf{3 a}) \ldots \ldots \ldots . . . .32$

Fig. S42. FT-IR spectrum of $\left[\mathrm{RuCl}(p\right.$-cymene $\left.)\left(\mathrm{S}_{2} \mathrm{C} \cdot \mathrm{IDip}\right)\right]\left[\mathrm{RuCl}_{3}(p\right.$-cymene $\left.)\right](\mathbf{3 b}) \ldots \ldots \ldots . . . .32$

Fig. S43. FT-IR spectrum of $\left[\mathrm{RuCl}(p\right.$-cymene $\left.)\left(\mathrm{S}_{2} \mathrm{C} \cdot \mathrm{ICy}\right)\right]\left[\mathrm{RuCl}_{3}(p\right.$-cymene $\left.)\right](\mathbf{3 c}) \ldots \ldots \ldots . . . . .33$

Fig. S44. FT-IR spectrum of $\left[\mathrm{RuCl}(p\right.$-cymene $\left.)\left(\mathrm{S}_{2} \mathrm{C} \cdot \mathrm{SIMes}\right)\right]\left[\mathrm{RuCl}_{3}(p\right.$-cymene $\left.)\right](\mathbf{3 d}) \ldots \ldots . . .33$

Fig. S45. FT-IR spectrum of $\left[\mathrm{RuCl}(p\right.$-cymene $\left.)\left(\mathrm{S}_{2} \mathrm{C} \cdot \mathrm{SIDip}\right)\right]\left[\mathrm{RuCl}_{3}(p\right.$-cymene $\left.)\right](\mathbf{3 e}) \ldots . . . . . .34$

Part 4 - Mass spectra 35

Fig. S46. HR-MS spectrum $\left(\mathrm{CH}_{3} \mathrm{CN}\right.$, positive mode) of $\left[\mathrm{RuCl}(p\right.$-cymene $\left.)\left(\mathrm{S}_{2} \mathrm{C} \cdot \mathrm{IMes}\right)\right] \mathrm{Cl}(\mathbf{2 a})$

Fig. S47. HR-MS spectrum $\left(\mathrm{CH}_{3} \mathrm{CN}\right.$, positive mode $)$ of $\left[\mathrm{RuCl}(p\right.$-cymene $\left.)\left(\mathrm{S}_{2} \mathrm{C} \cdot \mathrm{IDip}\right)\right] \mathrm{Cl}(\mathbf{2} \mathbf{b})$

Fig. S48. HR-MS spectrum $\left(\mathrm{CH}_{3} \mathrm{CN}\right.$, positive mode $)$ of $\left[\mathrm{RuCl}(p\right.$-cymene $\left.)\left(\mathrm{S}_{2} \mathrm{C} \cdot \mathrm{ICy}\right)\right] \mathrm{Cl}(\mathbf{2} \mathrm{c})$

Fig. S49. HR-MS spectrum $\left(\mathrm{CH}_{3} \mathrm{CN}\right.$, positive mode) of $\left[\mathrm{RuCl}(p\right.$-cymene $\left.)\left(\mathrm{S}_{2} \mathrm{C} \cdot \mathrm{SIMes}\right)\right] \mathrm{Cl}$

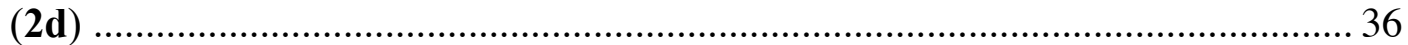

Fig. S50. HR-MS spectrum $\left(\mathrm{CH}_{3} \mathrm{CN}\right.$, positive mode $)$ of $\left[\mathrm{RuCl}(p\right.$-cymene $\left.)\left(\mathrm{S}_{2} \mathrm{C} \cdot \mathrm{SIDip}\right)\right] \mathrm{Cl}$ (2e)

Fig. S51. HR-MS spectrum $\left(\mathrm{CH}_{3} \mathrm{CN}\right.$, positive mode $)$ of $\left[\mathrm{RuCl}(p\right.$-cymene $\left.)\left(\mathrm{S}_{2} \mathrm{C} \cdot \mathrm{IMes}\right)\right]$ -

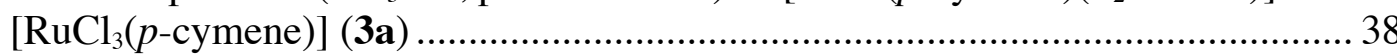

Fig. S52. HR-MS spectrum $\left(\mathrm{CH}_{3} \mathrm{CN}\right.$, negative mode) of $\left[\mathrm{RuCl}(p\right.$-cymene $\left.)\left(\mathrm{S}_{2} \mathrm{C} \cdot \mathrm{IMes}\right)\right]$ -

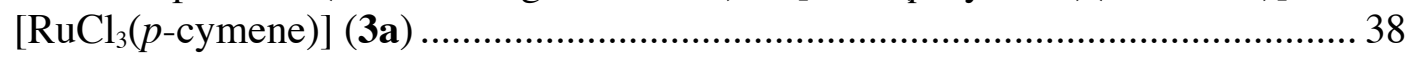

Fig. S53. HR-MS spectrum $\left(\mathrm{CH}_{3} \mathrm{CN}\right.$, positive mode) of $\left[\mathrm{RuCl}(p\right.$-cymene $\left.)\left(\mathrm{S}_{2} \mathrm{C} \cdot \mathrm{IDip}\right)\right]-$

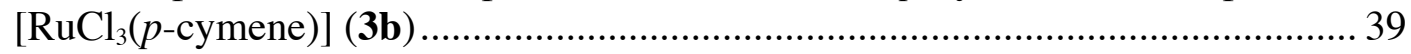

Fig. S54. HR-MS spectrum $\left(\mathrm{CH}_{3} \mathrm{CN}\right.$, negative mode $)$ of $\left[\mathrm{RuCl}(p\right.$-cymene $\left.)\left(\mathrm{S}_{2} \mathrm{C} \cdot \mathrm{IDip}\right)\right]$ -

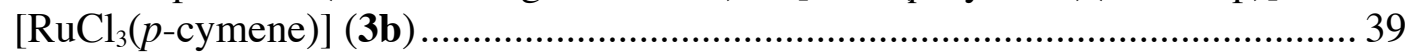

Fig. S55. HR-MS spectrum $\left(\mathrm{CH}_{3} \mathrm{CN}\right.$, positive mode $)$ of $\left[\mathrm{RuCl}(p\right.$-cymene $\left.)\left(\mathrm{S}_{2} \mathrm{C} \cdot \mathrm{ICy}\right)\right]$ $\left[\mathrm{RuCl}_{3}(p\right.$-cymene $\left.)\right](\mathbf{3 c})$. 40

Fig. S56. HR-MS spectrum $\left(\mathrm{CH}_{3} \mathrm{CN}\right.$, negative mode) of $\left[\mathrm{RuCl}(p\right.$-cymene $\left.)\left(\mathrm{S}_{2} \mathrm{C} \cdot \mathrm{ICy}\right)\right]$ $\left[\mathrm{RuCl}_{3}(p\right.$-cymene $\left.)\right](\mathbf{3 c})$. 40

Fig. S57. HR-MS spectrum $\left(\mathrm{CH}_{3} \mathrm{CN}\right.$, positive mode $)$ of $\left[\mathrm{RuCl}(p\right.$-cymene $\left.)\left(\mathrm{S}_{2} \mathrm{C} \cdot \mathrm{SIMes}\right)\right]$ $\left[\mathrm{RuCl}_{3}(p\right.$-cymene $\left.)\right](\mathbf{3 d})$...

Fig. S58. HR-MS spectrum $\left(\mathrm{CH}_{3} \mathrm{CN}\right.$, negative mode $)$ of $\left[\mathrm{RuCl}(p\right.$-cymene $\left.)\left(\mathrm{S}_{2} \mathrm{C} \cdot \mathrm{SIMes}\right)\right]-$

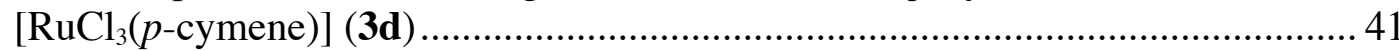

Fig. S59. HR-MS spectrum $\left(\mathrm{CH}_{3} \mathrm{CN}\right.$, positive mode) of $\left[\mathrm{RuCl}(p\right.$-cymene $\left.)\left(\mathrm{S}_{2} \mathrm{C} \cdot \mathrm{SIDip}\right)\right]-$ $\left[\mathrm{RuCl}_{3}(p\right.$-cymene $\left.)\right](\mathbf{3 e})$ 
Fig. S60. HR-MS spectrum $\left(\mathrm{CH}_{3} \mathrm{CN}\right.$, negative mode $)$ of $\left[\mathrm{RuCl}(p\right.$-cymene $\left.)\left(\mathrm{S}_{2} \mathrm{C} \cdot \mathrm{SIDip}\right)\right]-$ $\left[\mathrm{RuCl}_{3}(p\right.$-cymene $\left.)\right](\mathbf{3 e})$

Part 5 - Crystallography

Fig. S61. Powder diffraction pattern recorded for the bulk sample obtained by crystallization of $\left[\mathrm{RuCl}(p\right.$-cymene $\left.)\left(\mathrm{S}_{2} \mathrm{C} \cdot \mathrm{ICy}\right)\right]\left[\mathrm{RuCl}_{3}(p\right.$-cymene $\left.)\right](\mathbf{3 c})$

Fig. S62. Molecular structure of $\left[\operatorname{RuCl}(p\right.$-cymene $\left.)\left(\mathrm{S}_{2} \mathrm{C} \cdot \mathrm{IMes}\right)\right] \mathrm{PF}_{6}(\mathbf{1 a})$ showing the H-bonds of the imidazolium protons

Fig. S63. ORTEP representation of $\left[\mathrm{RuCl}(p\right.$-cymene $\left.)\left(\mathrm{S}_{2} \mathrm{C} \cdot \mathrm{IMes}\right)\right]\left[\mathrm{RuCl}_{3}(p\right.$-cymene $\left.)\right]$ cocrystallized with water $\left(\mathbf{3 a} \cdot \mathrm{H}_{2} \mathrm{O}\right)$

Fig. S64. ORTEP representation of $\left[\mathrm{RuCl}(p\right.$-cymene $\left.)\left(\mathrm{S}_{2} \mathrm{C} \cdot \mathrm{SIMes}\right)\right]\left[\mathrm{RuCl}_{3}(p\right.$-cymene $\left.)\right]$ cocrystallized with water $\left(2(\mathbf{3 d}) \cdot 3\left(\mathrm{H}_{2} \mathrm{O}\right)\right)$

Fig. S65. ORTEP representation of $\left[\mathrm{RuCl}(p\right.$-cymene $\left.)\left(\mathrm{S}_{2} \mathrm{C} \cdot \mathrm{IMes}\right)\right] \mathrm{Cl}$ co-crystallized with water $\left(\mathbf{2} \mathbf{a} \cdot \mathrm{H}_{2} \mathrm{O}\right)$

Fig. S66. ORTEP representation of $\left[\mathrm{RuCl}(p\right.$-cymene $\left.)\left(\mathrm{S}_{2} \mathrm{C} \cdot \mathrm{ICy}\right)\right] \mathrm{Cl}$ co-crystallized with water $\left(2(2 \mathrm{c}) \cdot 9.75\left(\mathrm{H}_{2} \mathrm{O}\right)\right)$

Table S1. Analysis of Short Ring-Interactions with Cg-Cg Distances $(\AA)$, and Angles (deg) in $\left[\mathrm{RuCl}(p\right.$-cymene $\left.)\left(\mathrm{S}_{2} \mathrm{C} \cdot \mathrm{IMes}\right)\right] \mathrm{Cl}(\mathbf{2 a}),\left[\mathrm{RuCl}(p\right.$-cymene $\left.)\left(\mathrm{S}_{2} \mathrm{C} \cdot \mathrm{IMes}\right)\right]\left[\mathrm{RuCl}_{3-}\right.$ (p-cymene)] (3a), and $\left[\mathrm{RuCl}(p\right.$-cymene $\left.)\left(\mathrm{S}_{2} \mathrm{C} \cdot \mathrm{SIMes}\right)\right]\left[\mathrm{RuCl}_{3}(p\right.$-cymene $\left.)\right](\mathbf{3 d}) \ldots . .48$

Table S2. Selected Bond Lengths ( $\AA$ ), and Angles (deg) for the Hydrogen Bonds Between the Counteranions and Solvent molecules Derived from the Crystal Structures of $\left[\mathrm{RuCl}(p\right.$-cymene $\left.)\left(\mathrm{S}_{2} \mathrm{C} \cdot \mathrm{IMes}\right)\right] \mathrm{Cl}(\mathbf{2 a}),\left[\mathrm{RuCl}(p\right.$-cymene $\left.)\left(\mathrm{S}_{2} \mathrm{C} \cdot \mathrm{IMes}\right)\right]\left[\mathrm{RuCl}_{3}(p\right.$-cymene)] (3a), $\left[\mathrm{RuCl}\left(p\right.\right.$-cymene) $\left.\left(\mathrm{S}_{2} \mathrm{C} \cdot \mathrm{ICy}\right)\right] \mathrm{Cl}(\mathbf{2 c})$, and $[\mathrm{RuCl}(p$-cymene)$\left.\left(\mathrm{S}_{2} \mathrm{C} \cdot \mathrm{SIMes}\right)\right]\left[\mathrm{RuCl}_{3}(p\right.$-cymene $\left.)\right](\mathbf{3 d})$.

Part 6 - Cytotoxicity

Fig. S67. In vitro cytotoxicity of $\left[\mathrm{RuCl}(p\right.$-cymene $\left.)\left(\mathrm{S}_{2} \mathrm{C} \cdot \mathrm{IMes}\right)\right] \mathrm{Cl}(\mathbf{2 a})$ against $\mathrm{K} 562$ cells measured after $72 \mathrm{~h}$

Fig. S68. In vitro cytotoxicity of $\left[\mathrm{RuCl}(p\right.$-cymene $\left.)\left(\mathrm{S}_{2} \mathrm{C} \cdot \mathrm{IDip}\right)\right] \mathrm{Cl}(\mathbf{2 b})$ against $\mathrm{K} 562$ cells measured after $72 \mathrm{~h}$

Fig. S69. In vitro cytotoxicity of $\left[\mathrm{RuCl}(p\right.$-cymene $\left.)\left(\mathrm{S}_{2} \mathrm{C} \cdot \mathrm{ICy}\right)\right] \mathrm{Cl}(\mathbf{2 c})$ against $\mathrm{K} 562$ cells measured after $72 \mathrm{~h}$

Fig. S70. In vitro cytotoxicity of $\left[\mathrm{RuCl}(p\right.$-cymene $\left.)\left(\mathrm{S}_{2} \mathrm{C} \cdot \mathrm{SIMes}\right)\right] \mathrm{Cl}(\mathbf{2 d})$ against $\mathrm{K} 562$ cells measured after $72 \mathrm{~h}$

Fig. S71. In vitro cytotoxicity of $\left[\mathrm{RuCl}(p\right.$-cymene $\left.)\left(\mathrm{S}_{2} \mathrm{C} \cdot \mathrm{SIDip}\right)\right] \mathrm{Cl}(\mathbf{2 e})$ against $\mathrm{K} 562$ cells measured after $72 \mathrm{~h}$

Fig. S72. In vitro cytotoxicity of $\left[\mathrm{RuCl}(p\right.$-cymene $\left.)\left(\mathrm{S}_{2} \mathrm{C} \cdot \mathrm{IMes}\right)\right]\left[\mathrm{RuCl}_{3}(\mathrm{p}\right.$-cymene $\left.)\right](\mathbf{3 a})$ against K562 cells measured after $72 \mathrm{~h}$.

Fig. S73. In vitro cytotoxicity of $\left[\mathrm{RuCl}(p\right.$-cymene $\left.)\left(\mathrm{S}_{2} \mathrm{C} \cdot \mathrm{IDip}\right)\right]\left[\mathrm{RuCl}_{3}(\mathrm{p}\right.$-cymene $\left.)\right](\mathbf{3 b})$ against K562 cells measured after $72 \mathrm{~h}$.

Fig. S74. In vitro cytotoxicity of $\left[\mathrm{RuCl}(p\right.$-cymene $\left.)\left(\mathrm{S}_{2} \mathrm{C} \cdot \mathrm{ICy}\right)\right]\left[\mathrm{RuCl}_{3}(\mathrm{p}\right.$-cymene $\left.)\right](3 \mathrm{c})$ against K562 cells measured after $72 \mathrm{~h}$.

Fig. S75. In vitro cytotoxicity of $\left[\mathrm{RuCl}(p\right.$-cymene $\left.)\left(\mathrm{S}_{2} \mathrm{C} \cdot \mathrm{SIMes}\right)\right]\left[\mathrm{RuCl}_{3}(\mathrm{p}\right.$-cymene $\left.)\right](3 \mathrm{~d})$ against K562 cells measured after $72 \mathrm{~h}$.

Fig. S76. In vitro cytotoxicity of $\left[\mathrm{RuCl}(p\right.$-cymene $\left.)\left(\mathrm{S}_{2} \mathrm{C} \cdot \mathrm{SIDip}\right)\right]\left[\mathrm{RuCl}_{3}(\mathrm{p}\right.$-cymene $\left.)\right](3 \mathbf{e})$ against K562 cells measured after $72 \mathrm{~h}$.

References 


\section{Part 1 - Experimental section}

\section{General Information}

Unless otherwise specified, all the syntheses were carried out under a dry nitrogen atmosphere using standard Schlenk techniques. Solvents were distilled from appropriate drying agents and deoxygenated prior to use. Imidazol(in)ium-2-dithiocarboxylates were synthesized according to published procedures. ${ }^{1}$ The $\left[\mathrm{RuCl}_{2}(p \text {-cymene })\right]_{2}$ dimer was purchased from Strem. Petroleum ether refers to the fraction of boiling point $40-60{ }^{\circ} \mathrm{C}$ and was purchased from VWR. All the other chemicals were obtained from Aldrich or TCI. ${ }^{1} \mathrm{H}$ and ${ }^{13} \mathrm{C}$ NMR spectra were recorded at $298 \mathrm{~K}$ with a Bruker DRX 400 spectrometer operating at 400.13 and $100.62 \mathrm{MHz}$, respectively. Chemical shifts are listed in parts per million downfield from TMS and are referenced from the solvent peaks or TMS. COSY, HSQC, and HMBC sequences were used for peak assignments. Infrared spectra were recorded with a Bruker Equinox 55 FT-IR spectrometer. Electrospray mass spectra were obtained using a Micromass LCT Premier instrument.

\section{Synthesis of Monometallic Complexes 2a-e}

A 10-mL glass vial equipped with a magnetic stirring bar and a rubber stopcock was charged with $\left[\mathrm{RuCl}_{2}(p \text {-cymene })\right]_{2}(0.0613 \mathrm{~g}, 0.1 \mathrm{mmol})$ and an imidazol(in)ium-2-dithiocarboxylate zwitterion $(0.2 \mathrm{mmol})$. Reagent grade dichloromethane $(2-3 \mathrm{~mL})$ taken straight from the bottle was added and the reaction mixture was stirred for $10 \mathrm{~min}$ at room temperature under a normal atmosphere. The solvent was evaporated under vacuum. The remaining solid was finely powdered, washed with petroleum ether $(5 \mathrm{~mL})$, and dried under high vacuum.

[RuCl( $\eta^{6}$-p-cymene $\left.)\left(\kappa^{2}-S, S^{\prime}-S_{2} C \cdot I M e s\right)\right] C l$ (2a). Dark red-purple microcrystalline powder $\left(0.137 \mathrm{~g}, 99.7 \%\right.$ yield). ${ }^{1} \mathrm{H}$ NMR $\left(400 \mathrm{MHz}, \mathrm{CDCl}_{3}\right): \delta 1.07\left(\mathrm{~d},{ }^{3} J_{\mathrm{HH}}=6.9 \mathrm{~Hz}, 6 \mathrm{H}, p\right.$-cym $\left.\mathrm{CH}\left(\mathrm{CH}_{3}\right)_{2}\right), 2.04\left(\mathrm{~s}, 12 \mathrm{H}\right.$, Mes $\left.o-\mathrm{CH}_{3}\right), 2.15\left(\mathrm{~s}, 3 \mathrm{H}, p\right.$-cym $\left.\mathrm{CH}_{3}\right), 2.38\left(\mathrm{~s}, 6 \mathrm{H}\right.$, Mes $p$ - $\left.\mathrm{CH}_{3}\right)$, $2.53\left(\mathrm{sept},{ }^{3} J_{\mathrm{HH}}=6.8 \mathrm{~Hz}, 1 \mathrm{H}, p\right.$-cym CH$\left.\left(\mathrm{CH}_{3}\right)_{2}\right), 5.52\left(\mathrm{~d},{ }^{3} J_{\mathrm{HH}}=6.1 \mathrm{~Hz}, 2 \mathrm{H}, p\right.$-cym $\left.\mathrm{CH}_{\mathrm{ar}}\right)$, $5.68\left(\mathrm{~d},{ }^{3} J_{\mathrm{HH}}=6.1 \mathrm{~Hz}, 2 \mathrm{H}, p\right.$-cym $\left.\mathrm{CH}_{\mathrm{ar}}\right), 7.02\left(\mathrm{~s}, 4 \mathrm{H}, \mathrm{Mes} m-\mathrm{CH}_{\mathrm{ar}}\right), 8.25 \mathrm{ppm}(\mathrm{s}, 2 \mathrm{H}, \mathrm{Im}$ $=\mathrm{CHN}) .{ }^{13} \mathrm{C} \mathrm{NMR}\left(101 \mathrm{MHz}, \mathrm{CDCl}_{3}\right): \delta 17.3,19.0,21.1,22.3,31.9,85.9,86.3,106.0,106.5$, 127.0, 129.7, 129.9, 134.1, 136.2, 141.6, $212.2 \mathrm{ppm}\left(\mathrm{CS}_{2}\right)$. ESI-MS $\left(\mathrm{CH}_{3} \mathrm{CN}\right.$, positive mode $)$ $\mathrm{m} / z$ calcd for $\mathrm{C}_{32} \mathrm{H}_{38} \mathrm{ClN}_{2} \mathrm{RuS}_{2}\left([\mathrm{M}]^{+}\right), 651.12071$; found, 651.12106 .

$\left[R u C l\left(\eta^{6}\right.\right.$-p-cymene $\left.)\left(\kappa^{2}-S, S^{\prime}-S_{2} C \cdot I D i p\right)\right] C l$ (2b). Dark red-purple microcrystalline powder (0.151 g, 98\% yield). ${ }^{1} \mathrm{H}$ NMR $\left(400 \mathrm{MHz}, \mathrm{CDCl}_{3}\right): \delta 1.06\left(\mathrm{~d},{ }^{3} J_{\mathrm{HH}}=6.9 \mathrm{~Hz}, 6 \mathrm{H}, p\right.$-cym $\left.\mathrm{CH}\left(\mathrm{CH}_{3}\right)_{2}\right), 1.21\left(\mathrm{~d},{ }^{3} J_{\mathrm{HH}}=6.5 \mathrm{~Hz}, 24 \mathrm{H}, \mathrm{Dip} \mathrm{CH}\left(\mathrm{CH}_{3}\right)_{2}\right), 2.09\left(\mathrm{~s}, 2 \mathrm{H}, p-\mathrm{cym} \mathrm{CH}_{3}\right), 2.33$ (sept, ${ }^{3} J_{\mathrm{HH}}=6.5 \mathrm{~Hz} 4 \mathrm{H}$, Dip CH$\left.\left(\mathrm{CH}_{3}\right)_{2}\right), 2.47$ (sept, ${ }^{3} J_{\mathrm{HH}}=7.0 \mathrm{~Hz}, 1 \mathrm{H}, p$-cym CH$\left.\left(\mathrm{CH}_{3}\right)_{2}\right)$, $5.44\left(\mathrm{~d},{ }^{3} J_{\mathrm{HH}}=6.1 \mathrm{~Hz}, 2 \mathrm{H}, p\right.$-cym $\left.\mathrm{CH}_{\mathrm{ar}}\right), 5.59\left(\mathrm{~d},{ }^{3} J_{\mathrm{HH}}=6.1 \mathrm{~Hz}, 2 \mathrm{H}, p-\mathrm{cym} \mathrm{CH}_{\mathrm{ar}}\right), 7.34(\mathrm{~d}$, ${ }^{3} J_{\mathrm{HH}}=7.8 \mathrm{~Hz}, 4 \mathrm{H}$, Dip $\left.m-\mathrm{CH}_{\mathrm{ar}}\right), 7.61\left(\mathrm{t},{ }^{3} J_{\mathrm{HH}}=7.8 \mathrm{~Hz}, 2 \mathrm{H}\right.$, Dip $\left.p-\mathrm{CH}_{\mathrm{ar}}\right), 8.39 \mathrm{ppm}(\mathrm{s}, 2 \mathrm{H}$, $\mathrm{Im}=\mathrm{CHN}) .{ }^{13} \mathrm{C} \mathrm{NMR}\left(101 \mathrm{MHz}, \mathrm{CDCl}_{3}\right): \delta 19.3,22.5,22.9,24.9,29.3,32.0,86.8,87.0$, $106.2,106.9,125.2,128.6,129.9,132.4,136.3,144.8,211.8 \mathrm{ppm}\left(\mathrm{CS}_{2}\right)$. ESI-MS $\left(\mathrm{CH}_{3} \mathrm{CN}\right.$, positive mode) $\mathrm{m} / z$ calcd for $\mathrm{C}_{38} \mathrm{H}_{50} \mathrm{ClN}_{2} \mathrm{RuS}_{2}\left([\mathrm{M}]^{+}\right)$, 735.21478; found, 735.22701 .

[RuCl( $\eta^{6}$-p-cymene $\left.)\left(\kappa^{2}-S, S^{\prime}-S_{2} C \cdot I C y\right)\right] C l(2 c)$. Dark yellow-green microcrystalline powder $(0.119 \mathrm{~g}, 97 \%$ yield $) .{ }^{1} \mathrm{H}$ NMR $\left(400 \mathrm{MHz}, \mathrm{CDCl}_{3}\right): \delta 1.19-1.45(\mathrm{~m}, 6 \mathrm{H}, \mathrm{Cy}), 1.33\left(\mathrm{~d},{ }^{3} J_{\mathrm{HH}}=\right.$ $6.9 \mathrm{~Hz}, 6 \mathrm{H}, p$-cym CH$\left.\left(\mathrm{CH}_{3}\right)_{2}\right), 1.60-1.75$ (m, $\left.2 \mathrm{H}, \mathrm{Cy}\right), 1.75-1.92$ (m, 8 H, Cy), $1.92-$ $2.06(\mathrm{~m}, 4 \mathrm{H}, \mathrm{Cy}), 2.38\left(\mathrm{~s}, 3 \mathrm{H}, p\right.$-cym $\left.\mathrm{CH}_{3}\right), 2.89$ (sept, ${ }^{3} J_{\mathrm{HH}}=6.7 \mathrm{~Hz}, 1 \mathrm{H}, p$-cym $\left.\mathrm{CH}\left(\mathrm{CH}_{3}\right)_{2}\right), 4.47\left(\mathrm{tt},{ }^{3} J_{\mathrm{HH}}=11.8,3.5 \mathrm{~Hz}, 2 \mathrm{H}, \mathrm{Cy} \mathrm{CHN}\right), 5.80\left(\mathrm{~d},{ }^{3} J_{\mathrm{HH}}=6.1 \mathrm{~Hz}, 2 \mathrm{H}, p\right.$-cym $\left.\mathrm{CH}_{\mathrm{ar}}\right), 6.03\left(\mathrm{~d},{ }^{3} \mathrm{~J}_{\mathrm{HH}}=6.1 \mathrm{~Hz}, 2 \mathrm{H}, p\right.$-cym $\left.\mathrm{CH}_{\mathrm{ar}}\right), 8.13 \mathrm{ppm}(\mathrm{s}, 2 \mathrm{H}, \mathrm{Im}=\mathrm{CHN}) .{ }^{13} \mathrm{C} \mathrm{NMR}(101$ 
$\left.\mathrm{MHz}, \mathrm{CDCl}_{3}\right): \delta 19.3,22.2,22.8,24.2,24.8,32.3,33.3,58.8,85.5,85.5,105.6,106.2,121.4$, $140.4,218.8 \mathrm{ppm}\left(\mathrm{CS}_{2}\right)$. ESI-MS $\left(\mathrm{CH}_{3} \mathrm{CN}\right.$, positive mode) $\mathrm{m} / z$ calcd for $\mathrm{C}_{26} \mathrm{H}_{38} \mathrm{ClN}_{2} \mathrm{RuS}$ $\left([\mathrm{M}]^{+}\right), 579.12055$; found, 579.12080.

[RuCl( $\eta^{6}$-p-cymene $\left.)\left(\kappa^{2}-S, S^{\prime}-S_{2} C \cdot S I M e s\right)\right] C l(2 d)$. Dark red-purple microcrystalline powder ( $0.136 \mathrm{~g}, 99 \%$ yield). ${ }^{1} \mathrm{H}$ NMR $\left(400 \mathrm{MHz}, \mathrm{CDCl}_{3}\right): \delta 0.96\left(\mathrm{~d},{ }^{3} J_{\mathrm{HH}}=6.9 \mathrm{~Hz}, 6 \mathrm{H}, p\right.$-cym $\left.\mathrm{CH}\left(\mathrm{CH}_{3}\right)_{2}\right), 2.11\left(\mathrm{~s}, 3 \mathrm{H}, p\right.$-cym $\left.\mathrm{CH}_{3}\right), 2.29$ (s, $6 \mathrm{H}$, Mes $\left.p-\mathrm{CH}_{3}\right), 2.33$ (s, $\left.12 \mathrm{H}, \mathrm{Mes} o-\mathrm{CH}_{3}\right)$, $2.42\left(\mathrm{sept},{ }^{3} J_{\mathrm{HH}}=6.9,1 \mathrm{H}, p\right.$-cym CH$\left.\left(\mathrm{CH}_{3}\right)_{2}\right), 4.64\left(\mathrm{~s}, 4 \mathrm{H}, \mathrm{Im} \mathrm{CH}_{2} \mathrm{~N}\right), 5.50\left(\mathrm{~d},{ }^{3} J_{\mathrm{HH}}=6.1 \mathrm{~Hz}\right.$, $2 \mathrm{H}, p$-cym $\left.\mathrm{CH}_{\mathrm{ar}}\right), 5.66\left(\mathrm{~d},{ }^{3} J_{\mathrm{HH}}=6.1 \mathrm{~Hz}, 2 \mathrm{H}, p\right.$-cym $\left.\mathrm{CH}_{\mathrm{ar}}\right), 6.90 \mathrm{ppm}\left(\mathrm{s}, 4 \mathrm{H}, \mathrm{Mes} m-\mathrm{CH}_{\mathrm{ar}}\right)$. ${ }^{13} \mathrm{C}$ NMR $\left(101 \mathrm{MHz}, \mathrm{CDCl}_{3}\right): \delta 18.0,19.0,21.0,22.1,22.3,31.9,51.8,85.7,86.3,106.5$, 107.2, 129.9, 129.9, 135.2, 140.4, 155.4, $213.9 \mathrm{ppm}\left(\mathrm{CS}_{2}\right)$. ESI-MS $\left(\mathrm{CH}_{3} \mathrm{CN}\right.$, positive mode) $\mathrm{m} / z$ calcd for $\mathrm{C}_{32} \mathrm{H}_{40} \mathrm{ClN}_{2} \mathrm{RuS}_{2}\left([\mathrm{M}]^{+}\right), 653.13636$; found, 653.14533 .

[RuCl( $\eta^{6}$-p-cymene $\left.)\left(\kappa^{2}-S, S^{\prime}-S_{2} C \cdot S I D i p\right)\right] C l$ (2e). Dark red-purple microcrystalline powder $(0.153 \mathrm{~g}, 99 \%$ yield $) .{ }^{1} \mathrm{H}$ NMR $\left(400 \mathrm{MHz}, \mathrm{CDCl}_{3}\right): \delta 1.02\left(\mathrm{~d},{ }^{3} J_{\mathrm{HH}}=6.9 \mathrm{~Hz}, 6 \mathrm{H}, p\right.$-cym $\left.\mathrm{CH}\left(\mathrm{CH}_{3}\right)_{2}\right), 1.28\left(\mathrm{~d},{ }^{3} J_{\mathrm{HH}}=6.8 \mathrm{~Hz}, 12 \mathrm{H}\right.$, Dip CH$\left.\left(\mathrm{CH}_{3}\right)_{2}\right), 1.34\left(\mathrm{~d},{ }^{3} J_{\mathrm{HH}}=6.8 \mathrm{~Hz}, 12 \mathrm{H}\right.$, Dip $\left.\mathrm{CH}\left(\mathrm{CH}_{3}\right)_{2}\right), 2.05\left(\mathrm{~s}, 3 \mathrm{H}, p\right.$-cym CH $\left.\mathrm{CH}_{3}\right), 2.42\left(\mathrm{sept},{ }^{3} J_{\mathrm{HH}}=6.9 \mathrm{~Hz}, 1 \mathrm{H}, p\right.$-cym $\left.\mathrm{CH}\left(\mathrm{CH}_{3}\right)_{2}\right), 2.95$ (sept, ${ }^{3} J_{\mathrm{HH}}=6.3 \mathrm{~Hz}, 4 \mathrm{H}$, Dip CH( $\left.\left(\mathrm{CH}_{3}\right)_{2}\right), 4.68\left(\mathrm{br} \mathrm{s}, 4 \mathrm{H}, \mathrm{Im} \mathrm{CH}_{2} \mathrm{~N}\right), 5.44\left(\mathrm{~d},{ }^{3} J_{\mathrm{HH}}=6.1 \mathrm{~Hz}, 2\right.$ $\mathrm{H}, p$-cym $\left.\mathrm{CH}_{\mathrm{ar}}\right), 5.59\left(\mathrm{~d},{ }^{3} J_{\mathrm{HH}}=6.1 \mathrm{~Hz}, 2 \mathrm{H}, p\right.$-cym $\left.\mathrm{CH}_{\mathrm{ar}}\right), 7.24\left(\mathrm{~d},{ }^{3} J_{\mathrm{HH}}=7.8 \mathrm{~Hz}, 4 \mathrm{H}, \mathrm{Dip} m-\right.$ $\left.\mathrm{CH}_{\mathrm{ar}}\right), 7.48 \mathrm{ppm}\left(\mathrm{t},{ }^{3} \mathrm{~J}_{\mathrm{HH}}=7.8 \mathrm{~Hz}, 2 \mathrm{H}\right.$, Dip $\left.p-\mathrm{CH}_{\mathrm{ar}}\right) .{ }^{13} \mathrm{C} \mathrm{NMR}\left(101 \mathrm{MHz}, \mathrm{CDCl}_{3}\right): \delta 19.4$, 22.5, 23.7, 25.6, 29.3, 31.9, 54.6, 87.6, 87.7, 105.8, 108.7, 125.3, 129.9, 131.4, 146.0, 152.5, $211.7 \mathrm{ppm}\left(\mathrm{CS}_{2}\right)$. ESI-MS $\left(\mathrm{CH}_{3} \mathrm{CN}\right.$, positive mode) $\mathrm{m} / z$ calcd for $\mathrm{C}_{38} \mathrm{H}_{52} \mathrm{ClN}_{2} \mathrm{RuS}_{2}\left([\mathrm{M}]^{+}\right)$, 737.23043; found, 737.24248.

\section{Synthesis of Bimetallic Complexes 3a-e}

A 10-mL glass vial equipped with a magnetic stirring bar and a rubber stopcock was charged with $\left[\mathrm{RuCl}_{2}(p \text {-cymene })\right]_{2}(0.0613 \mathrm{~g}, 0.1 \mathrm{mmol})$ and an imidazol(in)ium-2-dithiocarboxylate zwitterion $(0.1 \mathrm{mmol})$. Reagent grade dichloromethane $(3 \mathrm{~mL})$ taken straight from the bottle was added and the reaction mixture was stirred for $10 \mathrm{~min}$ at room temperature under a normal atmosphere. The solvent was evaporated under vacuum. The remaining solid was washed with petroleum ether $(3 \times 10 \mathrm{~mL})$ and dried under high vacuum.

[RuCl( $\eta^{6}$-p-cymene $\left.)\left(\kappa^{2}-S, S^{\prime}-S_{2} C \cdot I M e s\right)\right]\left[R u C l_{3}\left(\eta^{6}\right.\right.$-p-cymene $\left.)\right]$ (3a). Dark red-purple microcrystalline powder $(0.099 \mathrm{~g}, 99.8 \%$ yield $) .{ }^{1} \mathrm{H} \mathrm{NMR}\left(400 \mathrm{MHz}, \mathrm{CDCl}_{3}\right): \delta 1.02\left(\mathrm{~d},{ }^{3} J_{\mathrm{HH}}=6.9\right.$ $\mathrm{Hz}, 6 \mathrm{H}, p$-cym $\left.\mathrm{CH}\left(\mathrm{CH}_{3}\right)_{2}\right), 1.25\left(\mathrm{t},{ }^{3} J_{\mathrm{HH}}=6.6 \mathrm{~Hz}, 6 \mathrm{H}, p\right.$-cym $\left.\mathrm{CH}\left(\mathrm{CH}_{3}\right)_{2}\right), 1.99(\mathrm{~s}, 12 \mathrm{H}$, Mes $o$ - $\left.\mathrm{CH}_{3}\right), 2.11$ (s, $3 \mathrm{H}, p$-cym $\left.\mathrm{CH}_{3}\right), 2.12$ (s, $1 \mathrm{H}, p$-cym $\left.\mathrm{CH}_{3}\right), 2.18$ (s, $2 \mathrm{H}, p$-cym $\left.\mathrm{CH}_{3}\right), 2.31$ $\left(\mathrm{s}, 6 \mathrm{H}\right.$, Mes $\left.p-\mathrm{CH}_{3}\right), 2.47\left(\mathrm{sept},{ }^{3} J_{\mathrm{HH}}=6.9 \mathrm{~Hz}, 1 \mathrm{H}, p\right.$-cym $\left.\mathrm{CH}\left(\mathrm{CH}_{3}\right)_{2}\right), 3.07\left(\mathrm{sept},{ }^{3} J_{\mathrm{HH}}=7.0\right.$ $\mathrm{Hz}, 1 \mathrm{H}, p$-cym $\left.\mathrm{CH}\left(\mathrm{CH}_{3}\right)_{2}\right), 5.12\left(\mathrm{~d},{ }^{3} \mathrm{~J}_{\mathrm{HH}}=5.8 \mathrm{~Hz}, 1 \mathrm{H}, p\right.$-cym $\left.\mathrm{CH}_{\mathrm{ar}}\right), 5.30\left(\mathrm{~d},{ }^{3} J_{\mathrm{HH}}=5.9 \mathrm{~Hz}, 1\right.$ $\mathrm{H}, p$-cym $\left.\mathrm{CH}_{\mathrm{ar}}\right), 5.36\left(\mathrm{~d},{ }^{3} J_{\mathrm{HH}}=5.8 \mathrm{~Hz}, 1 \mathrm{H}, p\right.$-cym $\left.\mathrm{CH}_{\mathrm{ar}}\right), 5.39\left(\mathrm{~d},{ }^{3} J_{\mathrm{HH}}=6.1 \mathrm{~Hz}, 2 \mathrm{H}, p\right.$-cym $\left.\mathrm{CH}_{\mathrm{ar}}\right), 5.43\left(\mathrm{~d},{ }^{3} J_{\mathrm{HH}}=5.9 \mathrm{~Hz}, 1 \mathrm{H}, p-\mathrm{cym} \mathrm{CH} \mathrm{CH}_{\mathrm{ar}}\right), 5.56\left(\mathrm{~d},{ }^{3} J_{\mathrm{HH}}=6.1 \mathrm{~Hz}, 2 \mathrm{H}, p-\mathrm{cym} \mathrm{CH} \mathrm{CH}_{\mathrm{ar}}\right)$, $6.93(\mathrm{~s}, 4 \mathrm{H}$, Mes $m$-CHar $), 8.30 \mathrm{ppm}(\mathrm{s}, 2 \mathrm{H}, \mathrm{Im}=\mathrm{CHN}) .{ }^{13} \mathrm{C}$ NMR $\left(101 \mathrm{MHz}, \mathrm{CDCl}_{3}\right): \delta$ $17.8,18.8,19.0,19.3,21.4,22.2$, 22.4 , 22.6, 30.6, 30.7, 32.3, 79.7, 80.6, 81.3, 81.7, 85.8, 86.3, 96.2, 96.8, 100.7, 101.3, 105.8, 106.7, 129.0, 130.0, 130.3, 134.7, 135.7, 141.6, 214.2 ppm $\left(\mathrm{CS}_{2}\right)$. IR (KBr): v $3063(\mathrm{~s}), 2956(\mathrm{~s}), 2916(\mathrm{~s}), 2867(\mathrm{~m}), 1606(\mathrm{~m}), 1544(\mathrm{~s}), 1484(\mathrm{~s})$, 1465 (s), 1424 (s), 1376 (m), 1230 (m), 1009 (m), $854(\mathrm{~m}), 722(\mathrm{~m}) \mathrm{cm}^{-1}$. ESI-MS $\left(\mathrm{CH}_{3} \mathrm{CN}\right.$, positive mode) $\mathrm{m} / z$ calcd for $\left[\mathrm{C}_{32} \mathrm{H}_{38} \mathrm{ClN}_{2} \mathrm{RuS}_{2}\right]^{+}\left(\left[\mathrm{M}_{\text {cation }}\right]^{+}\right), 651.12071$; found, 651.11968 . ESI-MS $\left(\mathrm{CH}_{3} \mathrm{CN}\right.$, negative mode) $\mathrm{m} / z$ calcd for $\left[\mathrm{C}_{10} \mathrm{H}_{14} \mathrm{Cl}_{3} \mathrm{Ru}\right]^{-}\left(\left[\mathrm{M}_{\text {anion }}\right]^{-}\right), 340.92101$; found, 340.92111 . 
[RuCl( $\eta^{6}$-p-cymene $\left.)\left(\kappa^{2}-S, S^{\prime}-S_{2} C \cdot I D i p\right)\right]\left[R u C l_{3}\left(\eta^{6}\right.\right.$-p-cymene $\left.)\right]$ (3b). Dark red-purple microcrystalline powder $\left(0.1018 \mathrm{~g}, 95 \%\right.$ yield). ${ }^{1} \mathrm{H}$ NMR $\left(400 \mathrm{MHz}, \mathrm{CDCl}_{3}\right): \delta 1.06\left(\mathrm{~d},{ }^{3} J_{\mathrm{HH}}=6.9\right.$ $\mathrm{Hz}, 6 \mathrm{H}, p$-cym $\left.\mathrm{CH}\left(\mathrm{CH}_{3}\right)_{2}\right), 1.11-1.38\left(\mathrm{~m}, 30 \mathrm{H}\right.$, $\mathrm{Dip} \mathrm{CH}\left(\mathrm{CH}_{3}\right)_{2}$ and $p$-cym $\left.\mathrm{CH}\left(\mathrm{CH}_{3}\right)_{2}\right), 2.09$ (s, $2 \mathrm{H}, p$-cym $\mathrm{CH}_{3}$ ), 2.16 (s, $3 \mathrm{H}, p$-cym $\mathrm{CH}_{3}$ ), 2.21 (s, $2 \mathrm{H}, p$-cym $\mathrm{CH}_{3}$ ), $2.26-2.41$ (m, 4 $\mathrm{H}$, Dip $\left.\mathrm{CH}\left(\mathrm{CH}_{3}\right)_{2}\right), 2.41-2.59\left(\mathrm{~m}, 1 \mathrm{H}, p\right.$-cym $\left.\mathrm{CH}\left(\mathrm{CH}_{3}\right)_{2}\right), 2.92$ (sept, ${ }^{3} J_{\mathrm{HH}}=6.8 \mathrm{~Hz}, 1 \mathrm{H}, p$ cym $\left.\mathrm{CH}\left(\mathrm{CH}_{3}\right)_{2}\right), 3.01-3.28\left(\mathrm{~m}, 1 \mathrm{H}, p\right.$-cym $\left.\mathrm{CH}\left(\mathrm{CH}_{3}\right)_{2}\right), 5.15\left(\mathrm{~d},{ }^{3} J_{\mathrm{HH}}=5.2 \mathrm{~Hz}, 1 \mathrm{H}, p\right.$-cym $\left.\mathrm{CH}_{\mathrm{ar}}\right), 5.34\left(\mathrm{~d},{ }^{3} J_{\mathrm{HH}}=5.8 \mathrm{~Hz}, 2 \mathrm{H}, p\right.$-cym $\left.\mathrm{CH}_{\mathrm{ar}}\right), 5.38\left(\mathrm{~d},{ }^{3} \mathrm{~J}_{\mathrm{HH}}=5.9 \mathrm{~Hz}, 2 \mathrm{H}, p\right.$-cym $\left.\mathrm{CH}_{\mathrm{ar}}\right)$, $5.48\left(\mathrm{~d},{ }^{3} J_{\mathrm{HH}}=5.8 \mathrm{~Hz}, 2 \mathrm{H}, p\right.$-cym $\left.\mathrm{CH}_{\mathrm{ar}}\right), 5.54\left(\mathrm{~d},{ }^{3} J_{\mathrm{HH}}=6.0 \mathrm{~Hz}, 2 \mathrm{H}, p\right.$-cym $\left.\mathrm{CH}_{\mathrm{ar}}\right), 7.31(\mathrm{~d}$, ${ }^{3} J_{\mathrm{HH}}=7.8 \mathrm{~Hz}, 4 \mathrm{H}$, Dip $\left.m-\mathrm{CH}_{\mathrm{ar}}\right), 7.57\left(\mathrm{t},{ }^{3} \mathrm{~J}_{\mathrm{HH}}=7.8 \mathrm{~Hz}, 2 \mathrm{H}\right.$, Dip $\left.p-\mathrm{CH}_{\mathrm{ar}}\right), 8.49 \mathrm{ppm}(\mathrm{s}, 2 \mathrm{H}, \mathrm{Im}$ $=\mathrm{CHN}) .{ }^{13} \mathrm{C}$ NMR $\left(101 \mathrm{MHz}, \mathrm{CDCl}_{3}\right): \delta 18.8,19.0,19.4,22.2,22.4,22.8,23.1,23.9,24.9$, 25.1, 29.0, 29.4, 30.7, 32.2, 79.6, 80.6, 81.3, 81.7, 86.7, 86.9, 96.8, 100.7, 101.2, 106.1, 106.8, 125.3 , 130.3, 130.6, 132.3, 136.0, 145.2, $213.2 \mathrm{ppm}\left(\mathrm{CS}_{2}\right)$. IR (KBr): v3054 (m), 2962 (s), 2926 (m), 2868 (m), 1624 (w), 1543 (s), 1468 (s), 1388 (m), 1001 (m), $802(\mathrm{~m}) \mathrm{cm}^{-1}$. ESI-MS $\left(\mathrm{CH}_{3} \mathrm{CN}\right.$, positive mode): $\mathrm{m} / \mathrm{z}$ calcd for $\left[\mathrm{C}_{32} \mathrm{H}_{38} \mathrm{ClN}_{2} \mathrm{RuS}_{2}\right]^{+}\left(\left[\mathrm{M}_{\text {cation }}\right]^{+}\right), 735.21478$; found, 735.21374. ESI-MS $\left(\mathrm{CH}_{3} \mathrm{CN}\right.$, negative mode): $\mathrm{m} / z$ calcd for $\left[\mathrm{C}_{10} \mathrm{H}_{14} \mathrm{Cl}_{3} \mathrm{Ru}\right]^{-}\left(\left[\mathrm{M}_{\text {anion }}\right]^{-}\right)$, 340.92101 ; found, 340.92113 .

[RuCl( $\eta^{6}$-p-cymene $\left.)\left(\kappa^{2}-S, S^{\prime}-S_{2} C \cdot I C y\right)\right]\left[R u C l_{3}\left(\eta^{6}\right.\right.$-p-cymene $\left.)\right](\mathbf{3 c})$. Dark yellow-green microcrystalline powder $(0.0910 \mathrm{~g}, 99 \%$ yield $) .{ }^{1} \mathrm{H}$ NMR $\left(400 \mathrm{MHz}, \mathrm{CDCl}_{3}\right): \delta 1.10-1.41(\mathrm{~m}, 21$ $\mathrm{H}, p$-cym $\mathrm{CH}\left(\mathrm{CH}_{3}\right)_{2}$ and $\left.\mathrm{Cy}\right), 1.53-2.02(\mathrm{~m}, 14 \mathrm{H}, \mathrm{Cy}), 2.11\left(\mathrm{~s}, 3 \mathrm{H}, p\right.$-cym $\left.\mathrm{CH}_{3}\right), 2.32$ (s, 3 $\mathrm{H}, p$-cym $\left.\mathrm{CH}_{3}\right), 2.67-3.01\left(\mathrm{~m}, 2 \mathrm{H}, p\right.$-cym $\left.\mathrm{CH}\left(\mathrm{CH}_{3}\right)_{2}\right), 5.30(\mathrm{~d}, J=5.9 \mathrm{~Hz}, 2 \mathrm{H}, p$-cym $\left.\mathrm{CH}_{\mathrm{ar}}\right), 5.43\left(\mathrm{~d}, J=5.8 \mathrm{~Hz}, 3 \mathrm{H}, p\right.$-cym $\left.\mathrm{CH}_{\mathrm{ar}}\right), 5.69\left(\mathrm{~d}, J=5.7 \mathrm{~Hz}, 1 \mathrm{H}, p\right.$-cym $\left.\mathrm{CH}_{\mathrm{ar}}\right), 5.91(\mathrm{~d}, J$ $=5.8 \mathrm{~Hz}, 1 \mathrm{H}, p$-cym $\left.\mathrm{CH}_{\mathrm{ar}}\right), 8.06 \mathrm{ppm}(\mathrm{s}, 2 \mathrm{H}, \mathrm{Im}=\mathrm{CHN}) \cdot{ }^{13} \mathrm{C} \mathrm{NMR}\left(101 \mathrm{MHz}, \mathrm{CDCl}_{3}\right): \delta$ $18.9,19.0,19.5,22.2,22.4,23.0,23.1,24.4,25.1,30.7,32.6,33.4,59.0,80.6,81.3,85.55$, 85.60, 96.8, 101.2, 105.8, 106.2, 122.6, 140.0, $220.2 \mathrm{ppm}\left(\mathrm{CS}_{2}\right)$. IR (KBr): $v 3054(\mathrm{~m}), 2931$ (s), 2858 (s), 1631 (w), 1562 (w), 1464 (s), 1449 (s), 1388 (m), 1375 (m), 1248 (m), 1201 (s), $1026(\mathrm{~s}) \mathrm{cm}^{-1}$. ESI-MS $\left(\mathrm{CH}_{3} \mathrm{CN}\right.$, positive mode): $\mathrm{m} / \mathrm{z}$ calcd for $\left[\mathrm{C}_{32} \mathrm{H}_{38} \mathrm{ClN}_{2} \mathrm{RuS}_{2}\right]^{+}\left(\left[\mathrm{M}_{\text {cation }}\right]^{+}\right)$, 579.12029; found, 579.12005. ESI-MS $\left(\mathrm{CH}_{3} \mathrm{CN}\right.$, negative mode $): \mathrm{m} / z$ calcd for $\left[\mathrm{C}_{10} \mathrm{H}_{14} \mathrm{Cl}_{3} \mathrm{Ru}\right]^{-}\left(\left[\mathrm{M}_{\text {anion }}\right]^{-}\right), 340.92101$; found, 340.92119 .

[RuCl( $\eta^{6}$-p-cymene $\left.)\left(\kappa^{2}-S, S^{\prime}-S_{2} C \cdot S I M e s\right)\right]\left[R_{u} C_{3}\left(\eta^{6}\right.\right.$-p-cymene $\left.)\right]$ (3d). Dark red-purple microcrystalline powder $(0.0965 \mathrm{~g}, 97 \%$ yield $) .{ }^{1} \mathrm{H} \mathrm{NMR}\left(400 \mathrm{MHz}, \mathrm{CDCl}_{3}\right): \delta 0.96\left(\mathrm{~d},{ }^{3} J_{\mathrm{HH}}=6.9\right.$ $\mathrm{Hz}, 6 \mathrm{H}, p$-cym $\left.\mathrm{CH}\left(\mathrm{CH}_{3}\right)_{2}\right), 1.28\left(\mathrm{t},{ }^{3} J_{\mathrm{HH}}=6.7 \mathrm{~Hz}, 6 \mathrm{H}, p\right.$-cym $\left.\mathrm{CH}\left(\mathrm{CH}_{3}\right)_{2}\right), 2.11(\mathrm{~s}, 3 \mathrm{H}, p$ cym $\left.\mathrm{CH}_{3}\right), 2.15$ (s, $2 \mathrm{H}, p$-cym $\left.\mathrm{CH}_{3}\right), 2.23\left(\mathrm{~s}, 1 \mathrm{H}, p\right.$-cym $\left.\mathrm{CH}_{3}\right), 2.27$ (s, $6 \mathrm{H}, \mathrm{Mes} p$ - $\mathrm{CH}_{3}$ ), $2.34\left(\mathrm{~s}, 12 \mathrm{H}\right.$, Mes $o$ - $\left.\mathrm{CH}_{3}\right), 2.37-2.49\left(\mathrm{~m}, 1 \mathrm{H}, p\right.$-cym $\left.\mathrm{CH}\left(\mathrm{CH}_{3}\right)_{2}\right), 2.91(\mathrm{~m}, 1 \mathrm{H}, p$-cym $\left.\mathrm{CH}\left(\mathrm{CH}_{3}\right)_{2}\right), 3.11\left(\mathrm{~m}, 1 \mathrm{H}, p\right.$-cym CH$\left.\left(\mathrm{CH}_{3}\right)_{2}\right), 4.64\left(\right.$ br s, $\left.4 \mathrm{H}, \mathrm{Im} \mathrm{CH}_{2} \mathrm{~N}\right), 5.17\left(\mathrm{~d},{ }^{3} J_{\mathrm{HH}}=5.6\right.$ $\mathrm{Hz}, 1 \mathrm{H}, p$-cym $\left.\mathrm{CH}_{\mathrm{ar}}\right), 5.33\left(\mathrm{~d},{ }^{3} J_{\mathrm{HH}}=5.8 \mathrm{~Hz}, 1 \mathrm{H}, p\right.$-cym $\left.\mathrm{CH}_{\mathrm{ar}}\right), 5.39\left(\mathrm{~d},{ }^{3} J_{\mathrm{HH}}=5.6 \mathrm{~Hz}, 3 \mathrm{H}\right.$, $p$-cym $\left.\mathrm{CH}_{\mathrm{ar}}\right), 5.47\left(\mathrm{~d},{ }^{3} J_{\mathrm{HH}}=5.8 \mathrm{~Hz}, 1 \mathrm{H}, p\right.$-cym $\left.\mathrm{CH}_{\mathrm{ar}}\right), 5.56\left(\mathrm{~d},{ }^{3} J_{\mathrm{HH}}=5.8 \mathrm{~Hz}, 2 \mathrm{H}, p\right.$-cym $\left.\mathrm{CH}_{\mathrm{ar}}\right), 6.87 \mathrm{ppm}\left(\mathrm{s}, 4 \mathrm{H}\right.$, Mes $\left.m-\mathrm{CH}_{\mathrm{ar}}\right) .{ }^{13} \mathrm{C} \mathrm{NMR}\left(101 \mathrm{MHz}, \mathrm{CDCl}_{3}\right): \delta 18.3,18.8,19.2,21.2$, 22.2, 22.4, 22.6, 30.6, 32.1, 53.1, 79.7, 80.6, 81.3, 81.5, 85.6, 86.2, 106.4, 107.2, 129.9, 130.4, 135.7, 140.3, 155.8, 215.8 ppm (CS $)$. IR (KBr): v $3045(\mathrm{w}), 2967(\mathrm{~m}), 2915(\mathrm{~m})$, 2865 (w), 1628 (w), 1607 (m), 1553 (s), 1510 (m), 1465 (s), 1378 (m), 1352 (w), 1284 (s), 1190 (w), 1031 (m), $851(\mathrm{~m}) \mathrm{cm}^{-1}$. ESI-MS $\left(\mathrm{CH}_{3} \mathrm{CN}\right.$, positive mode): $\mathrm{m} / \mathrm{z}$ calcd for $\left[\mathrm{C}_{32} \mathrm{H}_{38} \mathrm{ClN}_{2} \mathrm{RuS}_{2}\right]^{+}\left(\left[\mathrm{M}_{\text {cation }}\right]^{+}\right), 653.13636$; found, 653.13590. ESI-MS $\left(\mathrm{CH}_{3} \mathrm{CN}\right.$, negative mode): $\mathrm{m} / z$ calcd for $\left[\mathrm{C}_{10} \mathrm{H}_{14} \mathrm{Cl}_{3} \mathrm{Ru}\right]^{-}\left(\left[\mathrm{M}_{\text {anion }}\right]^{-}\right), 340.92101$; found, 340.92110 .

[RuCl( $\eta^{6}$-p-cymene $\left.)\left(\kappa^{2}-S, S^{\prime}-S_{2} C \cdot S I D i p\right)\right]\left[R u C l_{3}\left(\eta^{6}\right.\right.$-p-cymene $\left.)\right]$ (3e). Dark red-purple microcrystalline powder $(0.1060 \mathrm{~g}, 98 \%$ yield $) .{ }^{1} \mathrm{H} \mathrm{NMR}\left(400 \mathrm{MHz}, \mathrm{CDCl}_{3}\right): \delta 0.99\left(\mathrm{~d},{ }^{3} J_{\mathrm{HH}}=6.9\right.$ $\mathrm{Hz}, 6 \mathrm{H}, p$-cym CH$\left.\left(\mathrm{CH}_{3}\right)_{2}\right), 1.12-1.29$ (m, $20 \mathrm{H}$, Dip $\left.\mathrm{CH}\left(\mathrm{CH}_{3}\right)_{2}\right), 1.28-1.43(\mathrm{~m}, 12 \mathrm{H}, p$ cym $\mathrm{CH}\left(\mathrm{CH}_{3}\right)_{2}$ and $\left.\operatorname{Dip~} \mathrm{CH}\left(\mathrm{CH}_{3}\right)_{2}\right), 2.01\left(\mathrm{~s}, 3 \mathrm{H}, p\right.$-cym $\left.\mathrm{CH}_{3}\right), 2.12\left(\mathrm{~s}, 2 \mathrm{H}, p\right.$-cym $\left.\mathrm{CH}_{3}\right), 2.18$ 
$\left(\mathrm{d},{ }^{3} J_{\mathrm{HH}}=7.7 \mathrm{~Hz}, 2 \mathrm{H}, p\right.$-cym $\left.\mathrm{CH}_{3}\right), 2.39\left(\mathrm{sept},{ }^{3} J_{\mathrm{HH}}=6.9 \mathrm{~Hz}, 1 \mathrm{H}, p\right.$-cym CH$\left.\left(\mathrm{CH}_{3}\right)_{2}\right), 2.76-$ $3.15\left(\mathrm{~m}, 5 \mathrm{H}, p\right.$-cym $\mathrm{CH}\left(\mathrm{CH}_{3}\right)_{2}$ and Dip $\left.\mathrm{CH}\left(\mathrm{CH}_{3}\right)_{2}\right), 4.65$ (br s, $\left.4 \mathrm{H}, \operatorname{Im~} \mathrm{CH}_{2} \mathrm{~N}\right), 5.11(\mathrm{~s}, 1 \mathrm{H}$, $p$-cym $\left.\mathrm{CH}_{\mathrm{ar}}\right), 5.30\left(\mathrm{~d},{ }^{3} J_{\mathrm{HH}}=5.9 \mathrm{~Hz}, 1 \mathrm{H}, p\right.$-cym $\left.\mathrm{CH}_{\mathrm{ar}}\right), 5.34\left(\mathrm{~d},{ }^{3} J_{\mathrm{HH}}=5.8 \mathrm{~Hz}, 3 \mathrm{H}, p\right.$-cym $\left.\mathrm{CH}_{\mathrm{ar}}\right), 5.44\left(\mathrm{~d},{ }^{3} J_{\mathrm{HH}}=5.9 \mathrm{~Hz}, 1 \mathrm{H}, p\right.$-cym $\left.\mathrm{CH}_{\mathrm{ar}}\right), 5.50\left(\mathrm{~d},{ }^{3} J_{\mathrm{HH}}=5.8 \mathrm{~Hz}, 2 \mathrm{H}, p-\mathrm{cym} \mathrm{CH}_{\mathrm{ar}}\right)$, $7.18\left(\mathrm{~d},{ }^{3} J_{\mathrm{HH}}=7.7 \mathrm{~Hz}, 4 \mathrm{H}\right.$, Dip $\left.m-\mathrm{CH}_{\mathrm{ar}}\right), 7.41 \mathrm{ppm}\left(\mathrm{t},{ }^{3} J_{\mathrm{HH}}=7.7 \mathrm{~Hz}, 2 \mathrm{H}\right.$, Dip $\left.p-\mathrm{CH}_{\mathrm{ar}}\right) .{ }^{13} \mathrm{C}$ NMR $\left(101 \mathrm{MHz} \mathrm{CDCl}_{3}\right): \delta 18.8,19.0,19.5,22.2,22.4,22.8,23.9,25.9,29.4,30.7,32.0$, 79.7, 80.6, 81.3, 81.5, 87.5, 87.6, 96.8, 101.2, 105.6, 108.7, 125.3, 130.3, 131.3, 146.4, 152.9, 213.3 ppm $\left(\mathrm{CS}_{2}\right)$. IR (KBr): v $3060(\mathrm{w}), 2961(\mathrm{~s}), 2925(\mathrm{~m}), 2867(\mathrm{~m}), 1589(\mathrm{~m}), 1541(\mathrm{~s})$, $1494(\mathrm{~m}), 1461(\mathrm{~s}), 1442(\mathrm{~s}), 1360(\mathrm{~m}), 1279(\mathrm{~m}), 1055(\mathrm{~m}), 803(\mathrm{~m}) \mathrm{cm}^{-1}$. ESI-MS $\left(\mathrm{CH}_{3} \mathrm{CN}\right.$, positive mode): $\mathrm{m} / z$ calcd for $\left[\mathrm{C}_{32} \mathrm{H}_{38} \mathrm{ClN}_{2} \mathrm{RuS}_{2}\right]^{+}\left(\left[\mathrm{M}_{\text {cation }}\right]^{+}\right)$, 737.23043; found, 737.22976. ESI-MS $\left(\mathrm{CH}_{3} \mathrm{CN}\right.$, negative mode): $\mathrm{m} / z$ calcd for $\left[\mathrm{C}_{10} \mathrm{H}_{14} \mathrm{Cl}_{3} \mathrm{Ru}\right]^{-}\left(\left[\mathrm{M}_{\text {anion }}\right]^{-}\right), 340.92101$; found, 340.92115.

\section{X-Ray Crystallography}

Data for complexes 3a and 3c were collected on a Bruker APEX II diffractometer using the Mo-K $\alpha$ radiation $(\lambda=0.71073 \AA)$ from a fine focus sealed tube source at $100 \mathrm{~K}$. Data for complexes 2a and 2c were collected on a Bruker D8 VENTURE PHOTON III-14 diffractometer using an Incoatec multilayer mirror monochromated with the $\mathrm{Cu}-\mathrm{K} \alpha$ radiation ( $\lambda=1.54178 \AA$ ) from a microfocus sealed tube source at $100 \mathrm{~K}$ and with a detector resolution of 7.3910 pixels $\mathrm{mm}^{-1}$. Computing data and reduction was made with the APEX II software for all the samples. ${ }^{2}$ Absorption corrections based on the multiscan method were applied. ${ }^{3}$ All the structures were solved using SIR2004. ${ }^{4}$ They were refined by full-matrix, least-squares based on $F^{2}$ using SHELXL. ${ }^{5}$ An empirical absorption correction was applied using SADABS. ${ }^{6}$ All non-hydrogen atoms were anisotropically refined and the hydrogen atom positions were calculated and refined using a riding model. Soft geometrical restraints were necessary in some cases to refine the water geometry. The occupation factor for disordered parts was refined as an independent free variable and was aggregated in function of the obtained values.

Crystal data for $\left[R u C l\left(\eta^{6}-p\right.\right.$-cymene $\left.)\left(\kappa^{2}-S, S^{\prime}-S_{2} C \cdot I M e s\right)\right] C l$ (2a) co-crystallized with water. Dark green crystal with dimensions $0.09 \times 0.06 \times 0.02 \mathrm{~mm}$ obtained by slow diffusion of petroleum ether in a $\mathrm{CH}_{2} \mathrm{Cl}_{2}$ solution saturated with water at $6{ }^{\circ} \mathrm{C}, \mathrm{C}_{32} \mathrm{H}_{38} \mathrm{ClN}_{2} \mathrm{RuS}_{2} \cdot \mathrm{Cl} \cdot \mathrm{H}_{2} \mathrm{O}$, $\mathrm{MW}=704.75 \mathrm{~g} \mathrm{~mol}^{-1}$, triclinic, $P-1, a=10.2923(5) \AA, b=11.7054(5) \AA, c=13.1163(6) \AA$, $\alpha=84.218(4)^{\circ}, \beta=89.441(3), \gamma=87.349(3), V=1570.45(12) \AA^{3}, Z=2, D_{\mathrm{x}}=1.49 \mathrm{~g} \mathrm{~cm}^{-3}$, $\mu(\mathrm{Cu} \mathrm{K \alpha})=7.065 \mathrm{~mm}^{-1}, 40392$ reflns collected, 6391 independent reflns $\left(R_{\text {int }}=0.09\right), 5728$ reflns with $I>2 \sigma(I), R_{1}$ (all data) $=0.032, w R_{2}$ (all data) $=0.054$, GOF on $F_{2}=1.06, \varrho_{\max }=$ $0.38 \mathrm{e} \AA^{-3}, \varrho_{\min }=-0.42 \mathrm{e} \AA^{-3}$.

Crystal data for $\left[R u C l\left(\eta^{6}-p\right.\right.$-cymene $\left.)\left(\kappa^{2}-S, S^{\prime}-S_{2} C \cdot I C y\right)\right] C l$ (2c) co-crystallized with water. Orange crystal with dimensions $0.09 \times 0.03 \times 0.02 \mathrm{~mm}$ obtained by slow diffusion of petroleum ether in a $\mathrm{CH}_{2} \mathrm{Cl}_{2}$ solution saturated with water at $6{ }^{\circ} \mathrm{C}$, $2\left(\mathrm{C}_{26} \mathrm{H}_{38} \mathrm{ClN}_{2} \mathrm{RuS}_{2}\right) \cdot 2 \mathrm{Cl} \cdot 9.75\left(\mathrm{H}_{2} \mathrm{O}\right), \quad \mathrm{MW}=1404.84 \mathrm{~g} \mathrm{~mol}{ }^{-1}$, monoclinic, $C 2 / c, a=$ 24.5061(9)) $\AA, b=9.7644(4) \AA, c=27.0470(9) \AA, \beta=94.544(2), V=6451.7(4) \AA^{3}, Z=4, D_{\text {x }}$ $=1.46 \mathrm{~g} \mathrm{~cm}^{-3}, \mu(\mathrm{Cu} \mathrm{K \alpha})=6.967 \mathrm{~mm}^{-1}, 89737$ reflns collected, 6600 independent reflns $\left(R_{\text {int }}\right.$ $=0.093), 5275$ reflns with $I>2 \sigma(I), R_{1}$ (all data) $=0.055, w R_{2}($ all data $)=0.111$, GOF on $F_{2}$ $=1.04, \varrho_{\max }=0.96 \mathrm{e} \AA^{-3}, \varrho_{\min }=-1.16 \mathrm{e} \AA^{-3}$.

Crystal data for $\left[R u C l\left(\eta^{6}-p\right.\right.$-cymene $\left.)\left(\kappa^{2}-S, S^{\prime}-S_{2} C \cdot I M e s\right)\right]\left[R u C l_{3}\left(\eta^{6}-p\right.\right.$-cymene $\left.)\right]$ (3a) cocrystallized with water. Orange crystal with dimensions $0.4 \times 0.06 \times 0.04 \mathrm{~mm}$ obtained by slow evaporation of an acetone solution containing a few drops of water at $6{ }^{\circ} \mathrm{C}$, 
$\mathrm{C}_{32} \mathrm{H}_{38} \mathrm{ClN}_{2} \mathrm{RuS}_{2} \cdot \mathrm{C}_{10} \mathrm{H}_{14} \mathrm{Cl}_{3} \mathrm{Ru} \cdot \mathrm{H}_{2} \mathrm{O}, \mathrm{MW}=1010.93 \mathrm{~g} \mathrm{~mol}^{-1}$, monoclinic, $P 2_{1} / n, a=11.822(3)$ $\AA, b=28.056(5) \AA, c=14.239(2) \AA, \beta=110.133(8)^{\circ}, V=4434.3(14) \AA^{3}, Z=4, D_{\mathrm{x}}=1.514 \mathrm{~g}$ $\mathrm{cm}^{-3}, \mu($ Mo K $\alpha)=1.05 \mathrm{~mm}^{-1}, 33992$ reflns collected, 8417 independent reflns $\left(R_{\text {int }}=0.098\right)$, 5528 reflns with $I>2 \sigma(I), R_{1}$ (all data) $=0.101, w R_{2}$ (all data) $=0.129$, GOF on $F_{2}=1.01$, $\varrho_{\max }=0.80$ e $\AA^{-3}, \varrho_{\min }=-0.82 \mathrm{e} \AA^{-3}$.

Crystal data for [RuCl( $\eta^{6}$-p-cymene $\left.)\left(\kappa^{2}-S, S^{\prime}-S_{2} C \cdot S I M e s\right)\right]\left[R u C l_{3}\left(\eta^{6}-p\right.\right.$-cymene $\left.)\right]$ (3d) cocrystallized with water. Orange crystal with dimensions $0.28 \times 0.08 \times 0.04 \mathrm{~mm}$ obtained by slow evaporation of a $\mathrm{CH}_{2} \mathrm{Cl}_{2}$ solution containing a few drops of water at $6{ }^{\circ} \mathrm{C}$, $2\left(\mathrm{C}_{32} \mathrm{H}_{40} \mathrm{ClN}_{2} \mathrm{RuS}_{2}\right) \cdot 2\left(\mathrm{C}_{10} \mathrm{H}_{14} \mathrm{Cl}_{3} \mathrm{Ru}\right) \cdot 3\left(\mathrm{H}_{2} \mathrm{O}\right), \mathrm{MW}=2043.91 \mathrm{~g} \mathrm{~mol}^{-1}$, orthorhombic, Pbca,$a=$ 20.292(2) $\AA, b=15.6905(17) \AA, c=27.772(3) \AA, V=8842.5(16) \AA^{3}, Z=4, D_{\mathrm{x}}=1.535 \mathrm{~g} \mathrm{~cm}^{-}$ ${ }^{3}, \mu($ Mo K $\alpha)=1.06 \mathrm{~mm}^{-1}, 112907$ reflns collected, 8088 independent reflns $\left(R_{\text {int }}=0.054\right)$, 4928 reflns with $I>2 \sigma(I), R_{1}$ (all data) $=0.054, w R_{2}$ (all data) $=0.129, \mathrm{GOF}$ on $F_{2}=1.01$, $\varrho_{\max }=0.87 \mathrm{e} \AA^{-3}, \varrho_{\min }=-0.87 \mathrm{e} \AA^{-3}$.

CCDC 2037518 (2a), 2037519 (2c), 2037521 (3a), and 2037520 (3d) contain the supplementary crystallographic data for this paper. These data can be obtained free of charge via www.ccdc.cam.ac.uk/data_request/cif, or by emailing data_request@ccdc.cam.ac.uk, or by contacting The Cambridge Crystallographic Data Centre, 12 Union Road, Cambridge CB2 1EZ, UK; fax: +44 1223336033.

\section{Solubility Tests}

NMR tubes were loaded with powdered complexes $\mathbf{2 a - e}$ or $\mathbf{3 a}-\mathbf{e}$ (5 $\mu \mathrm{mol}$ each). Distilled water was added in small portions $(0.05 \mathrm{~mL})$ at room temperature with a glass microsyringe. The tubes were vigorously shaken after every addition. The process was repeated until complete dissolution of the solids.

\section{Cell Culture and Viability Assay}

Human leukemia K562 cells and mice splenocytes were cultured in RPMI 1640 and DMEM (Life Technologies, Waltham, MA, USA), respectively. Both culture media were supplemented with $10 \%$ heat-inactivated Fetal Bovine Serum (FBS; Life technologies), $1 \%$ glutamine, and $1 \%$ of penicillin-streptomycin (Life technologies) and maintained at $37{ }^{\circ} \mathrm{C}$ under $5 \% \mathrm{CO}_{2}$ in a humidified atmosphere. K562 Cells were passaged at least 5 times before any experiment. For the determination of cell densities, the cell counting was done using a Neubauer counting chamber with trypan blue staining $(0.4 \%$ in PBS; $\mathrm{pH}=7.4$; Life technologies). Stock solutions of the complexes were prepared in dimethyl sulfoxide (DMSO) or water at a concentration of $20 \mathrm{mg} / \mathrm{mL}$ and kept in a cold room at $4{ }^{\circ} \mathrm{C}$ before use. Due to the cytotoxicity of DMSO, its final concentration was limited to $1 \%$. To study the effect of complexes on the cell viability, a dose-dependent assay for all complexes was performed for three days using the CellTiter-Glo Luminescent Cell Viability Assay (Promega) as previously reported. ${ }^{7,8}$ Briefly, both K562 and splenocyte cells were incubated in the presence or absence of complexes for 3 days at $37{ }^{\circ} \mathrm{C}$ in a humidified $5 \% \mathrm{CO}_{2}$ atmosphere in 96-well plates. After 3 days of incubation, the cells were allowed to equilibrate at room temperature for approximately $30 \mathrm{~min}$ before adding $100 \mu \mathrm{L}$ of the CellTiter-Glo reagent to each well. The culture plate was then covered with an aluminum sheet to protect it from light and shaken for $2 \mathrm{~min}$ on an orbital shaker to induce cell lysis. It was further incubated for $10 \mathrm{~min}$ at room temperature for the stabilization of the luminescent signal before reading the luminescence on a Berthold Technologies luminometer (XS3LB 960). 
The cell viability (\%) was calculated by using the following formula:

Viability $(\%)=[$ RLU (treated cells) $/$ RLU (vehicle-treated cells) $] \times 100$

The selectivity index (SI) was calculated by using the following formula:

$\mathrm{SI}=\left(\mathrm{CC}_{50}\right.$ Splenocytes $) /\left(\mathrm{IC}_{50} \mathrm{~K} 562\right)$ 


\section{Part 2 - NMR spectra}

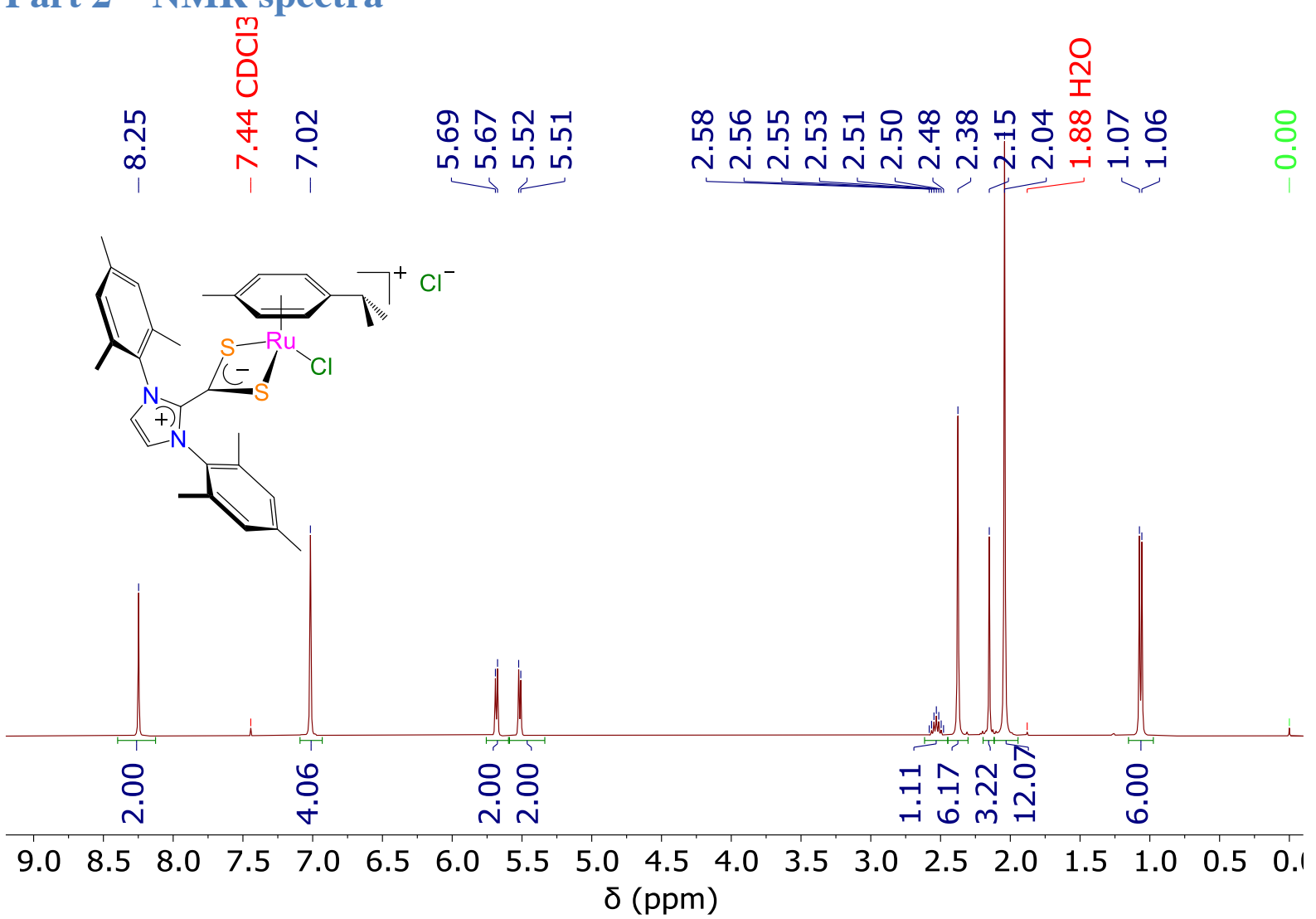

Fig. S1. ${ }^{1} \mathrm{H}$ NMR spectrum $\left(400 \mathrm{MHz}, \mathrm{CDCl}_{3}, 298 \mathrm{~K}\right)$ of $\left[\mathrm{RuCl}(p\right.$-cymene $\left.)\left(\mathrm{S}_{2} \mathrm{C} \cdot \mathrm{IMes}\right)\right] \mathrm{Cl}$ (2a)

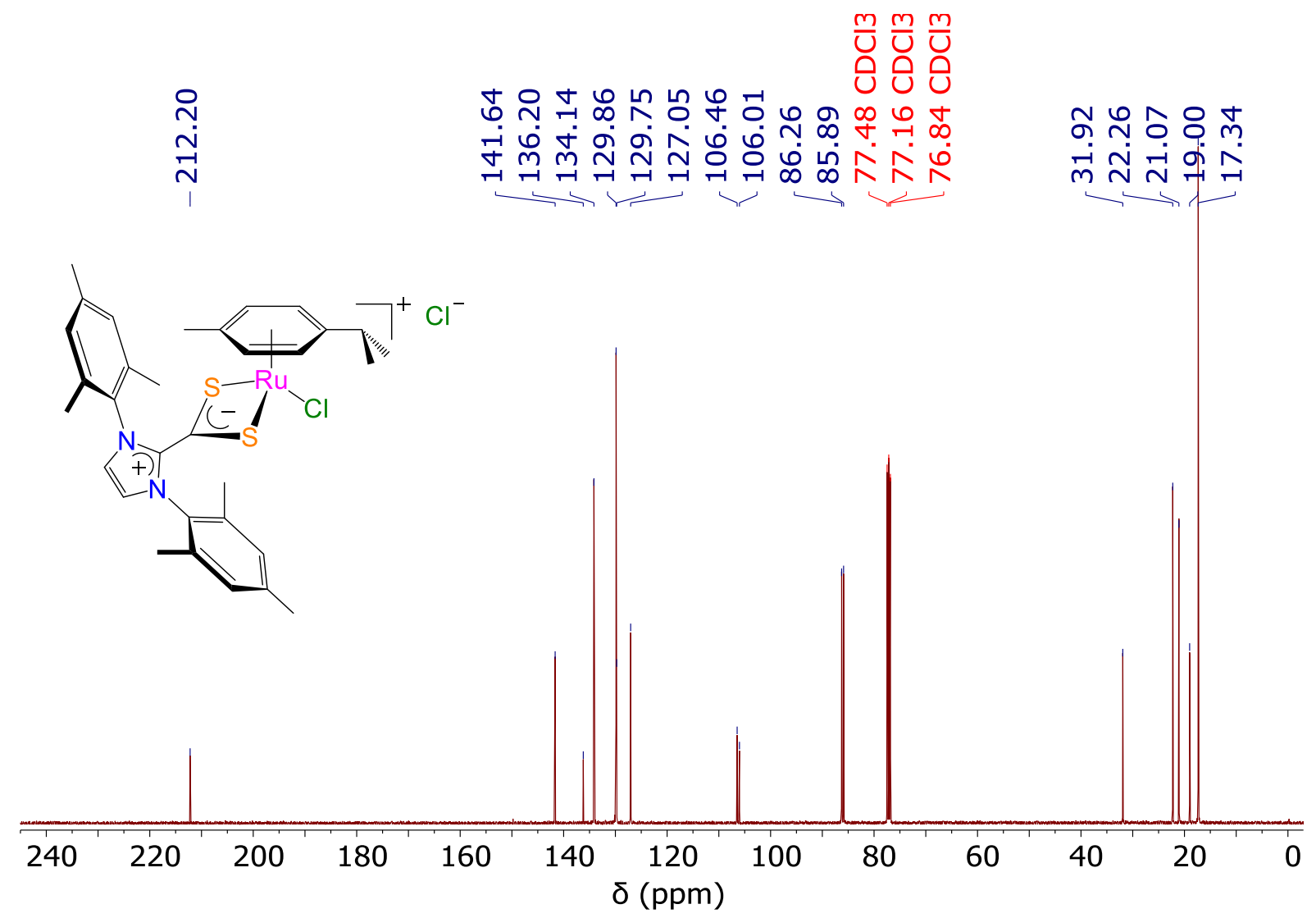


Fig. S2. ${ }^{13} \mathrm{C}\left\{{ }^{1} \mathrm{H}\right\} \mathrm{NMR}$ spectrum $\left(101 \mathrm{MHz}, \mathrm{CDCl}_{3}, 298 \mathrm{~K}\right)$ of $[\mathrm{RuCl}(p$-cymene)$\left.\left(\mathrm{S}_{2} \mathrm{C} \cdot \mathrm{IMes}\right)\right] \mathrm{Cl}$ (2a)

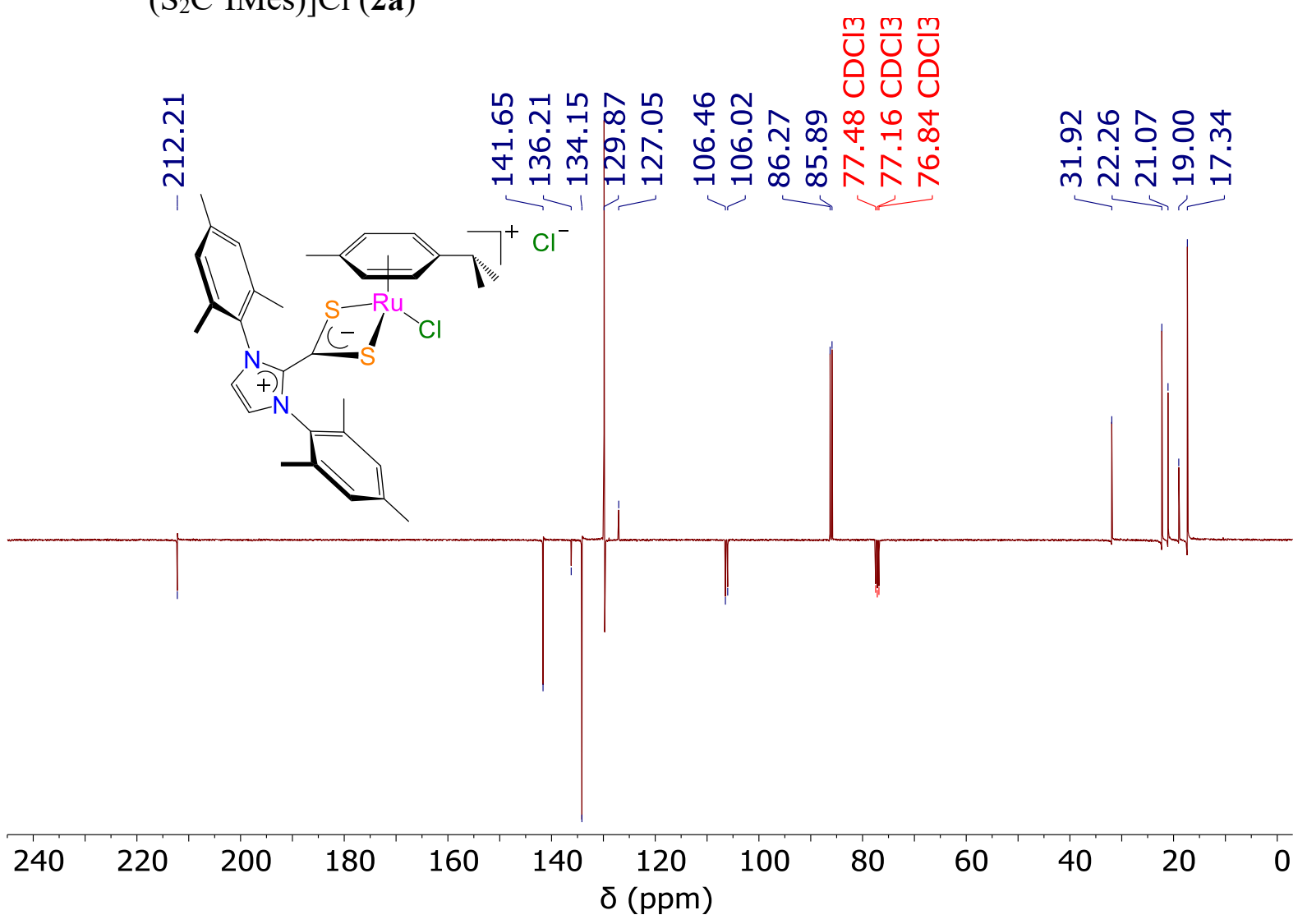

Fig. S3. ${ }^{13} \mathrm{C}\left\{{ }^{1} \mathrm{H}\right\}$ APT NMR spectrum (101 MHz, $\left.\mathrm{CDCl}_{3}, 298 \mathrm{~K}\right)$ of $[\mathrm{RuCl}(p$-cymene)$\left.\left(\mathrm{S}_{2} \mathrm{C} \cdot \mathrm{IMes}\right)\right] \mathrm{Cl}$ (2a)

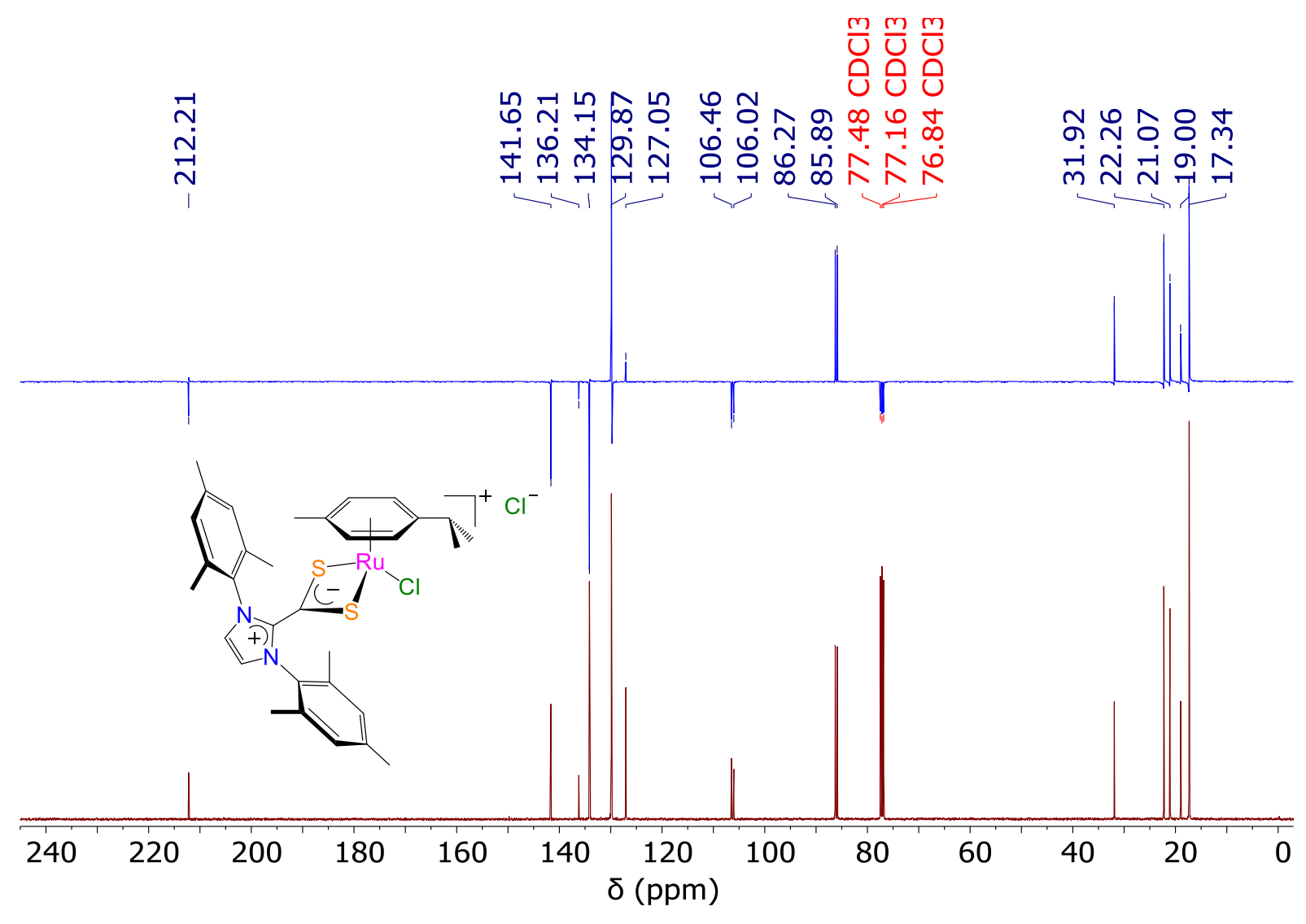


Fig. S4. ${ }^{13} \mathrm{C} \mathrm{CPD}$ and APT NMR spectra (101 $\left.\mathrm{MHz}, \mathrm{CDCl}_{3}, 298 \mathrm{~K}\right)$ of $[\mathrm{RuCl}(p$-cymene)$\left.\left(\mathrm{S}_{2} \mathrm{C} \cdot \mathrm{IMes}\right)\right] \mathrm{Cl}(\mathbf{2 a})$<smiles>[O]</smiles>

곤

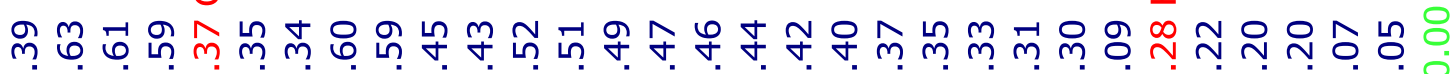

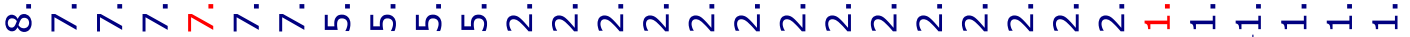
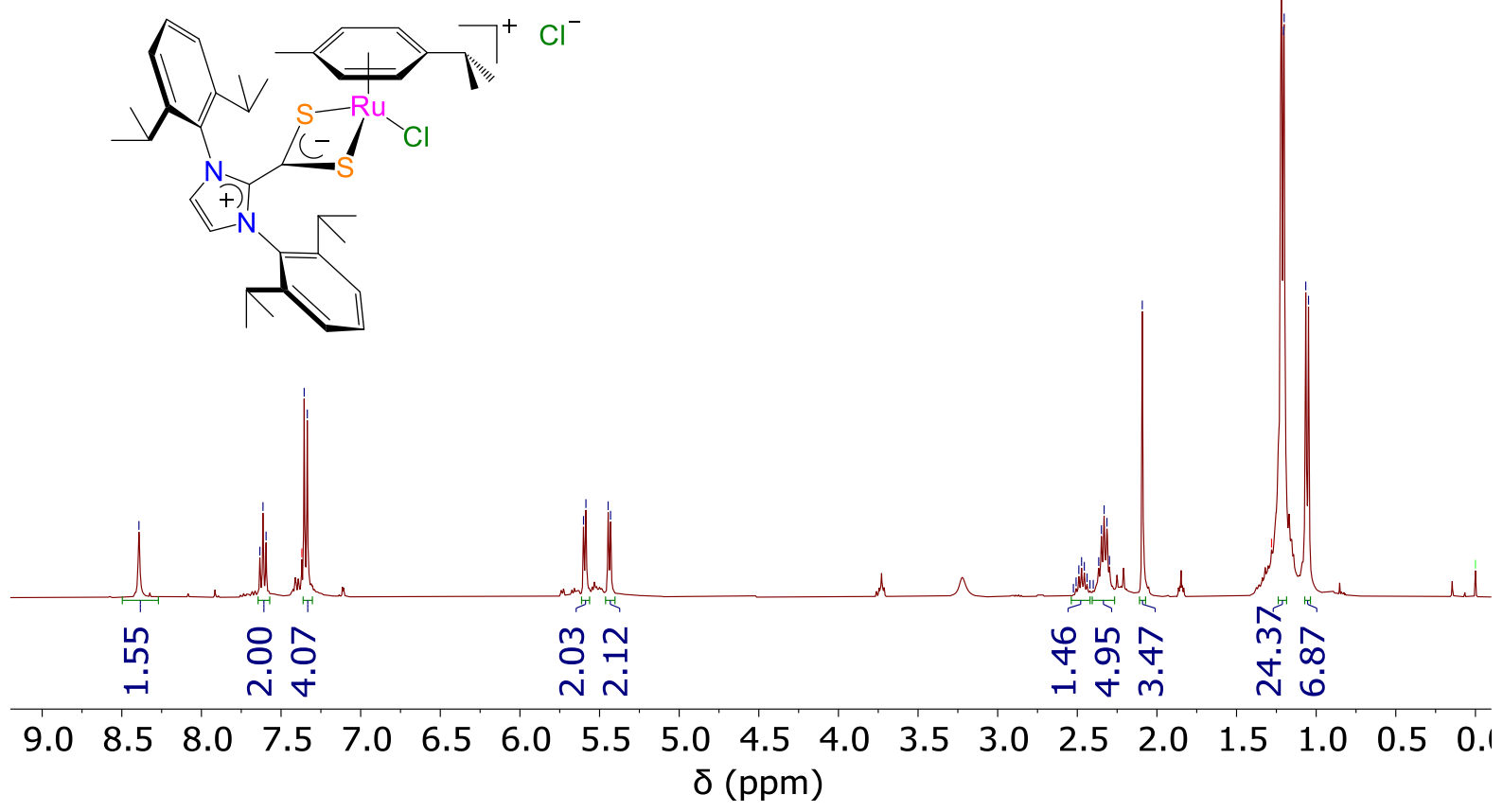

Fig. S5. ${ }^{1} \mathrm{H}$ NMR spectrum $\left(400 \mathrm{MHz}, \mathrm{CDCl}_{3}, 298 \mathrm{~K}\right)$ of $\left[\mathrm{RuCl}(p\right.$-cymene $\left.)\left(\mathrm{S}_{2} \mathrm{C} \cdot \mathrm{IDip}\right)\right] \mathrm{Cl}$ (2b)

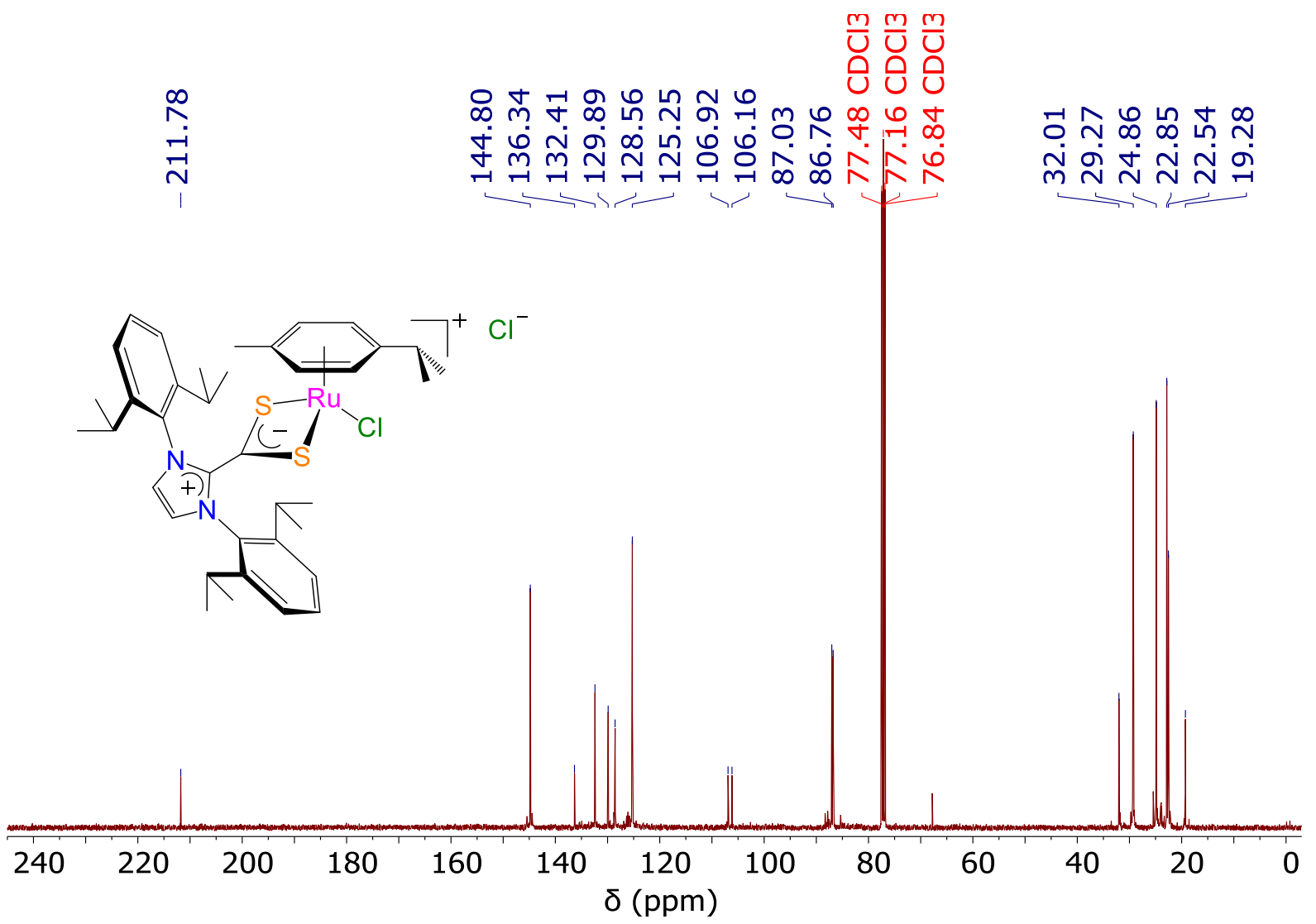


Fig. S6. ${ }^{13} \mathrm{C}\left\{{ }^{1} \mathrm{H}\right\} \mathrm{NMR}$ spectrum $\left(101 \mathrm{MHz}, \mathrm{CDCl}_{3}, 298 \mathrm{~K}\right)$ of $[\mathrm{RuCl}(p$-cymene)$\left.\left(\mathrm{S}_{2} \mathrm{C} \cdot \mathrm{IDip}\right)\right] \mathrm{Cl}(\mathbf{2 b})$

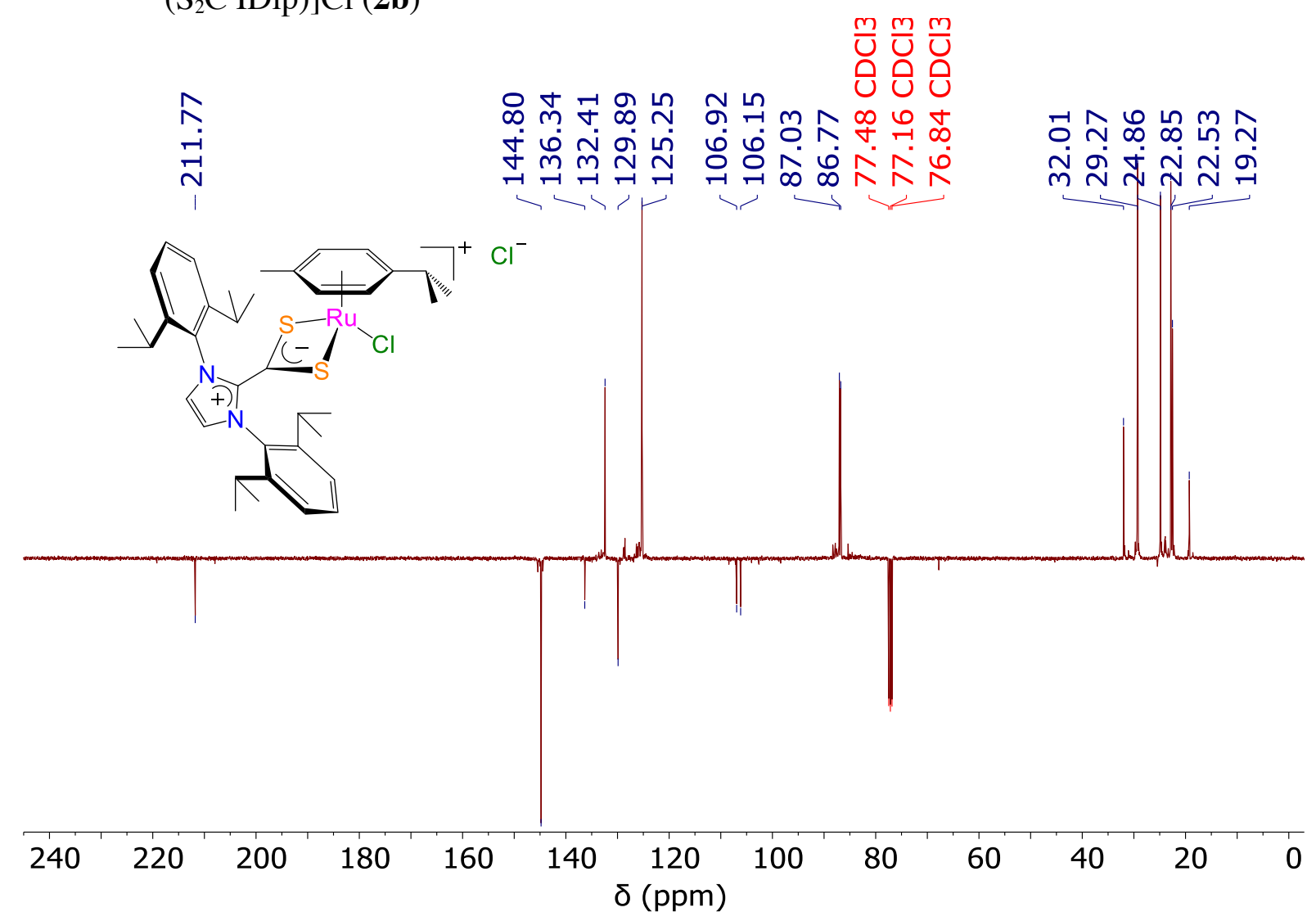

Fig. S7. ${ }^{13} \mathrm{C}\left\{{ }^{1} \mathrm{H}\right\}$ APT NMR spectrum (101 MHz, $\left.\mathrm{CDCl}_{3}, 298 \mathrm{~K}\right)$ of $[\mathrm{RuCl}(p$-cymene)$\left.\left(\mathrm{S}_{2} \mathrm{C} \cdot \mathrm{IDip}\right)\right] \mathrm{Cl}(\mathbf{2 b})$

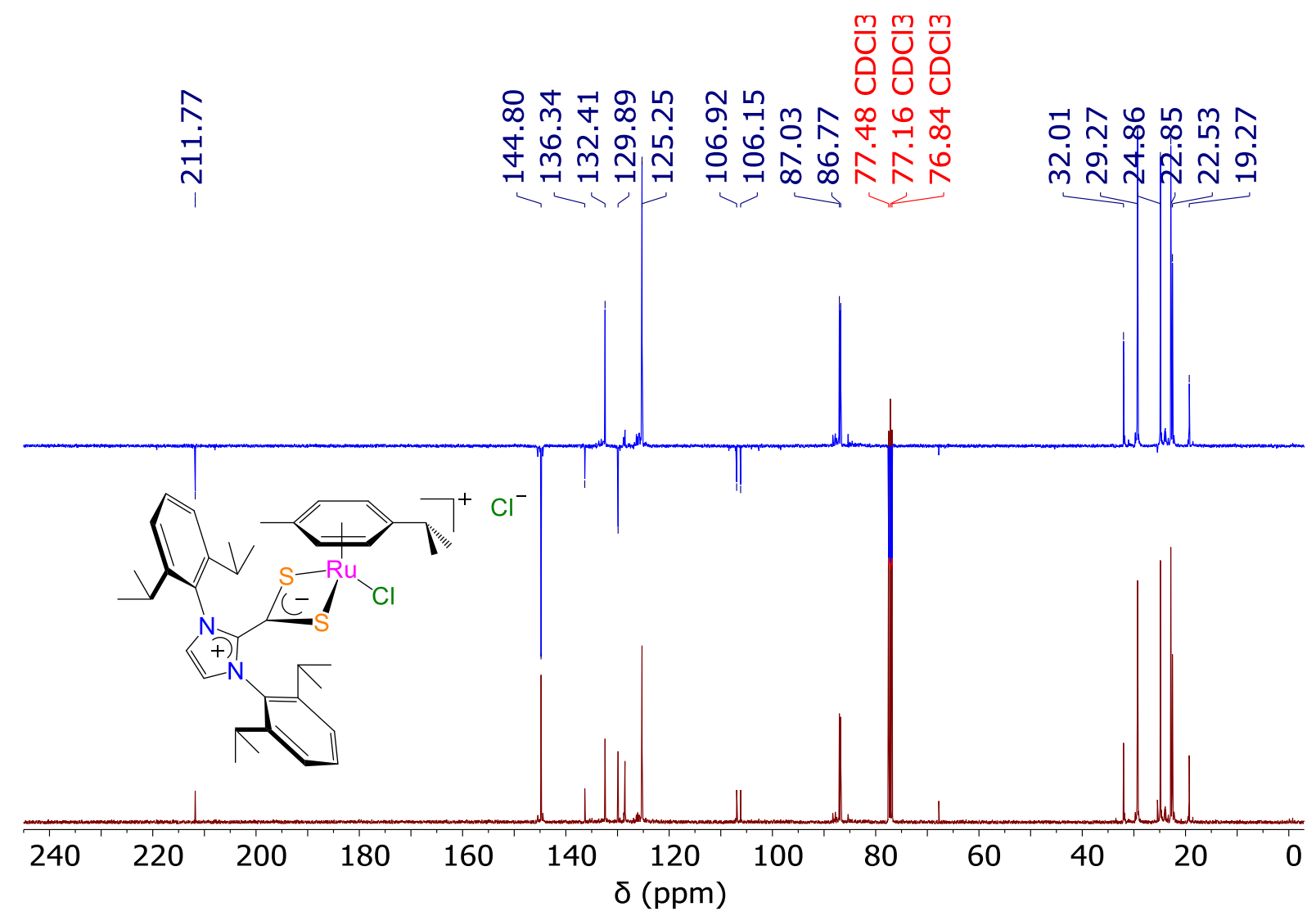


Fig. S8. ${ }^{13} \mathrm{C} \mathrm{CPD}$ and APT NMR spectra $\left(101 \mathrm{MHz}, \mathrm{CDCl}_{3}, 298 \mathrm{~K}\right)$ of $[\mathrm{RuCl}(p$-cymene $)$ $\left.\left(\mathrm{S}_{2} \mathrm{C} \cdot \mathrm{IDip}\right)\right] \mathrm{Cl}(\mathbf{2 b})$
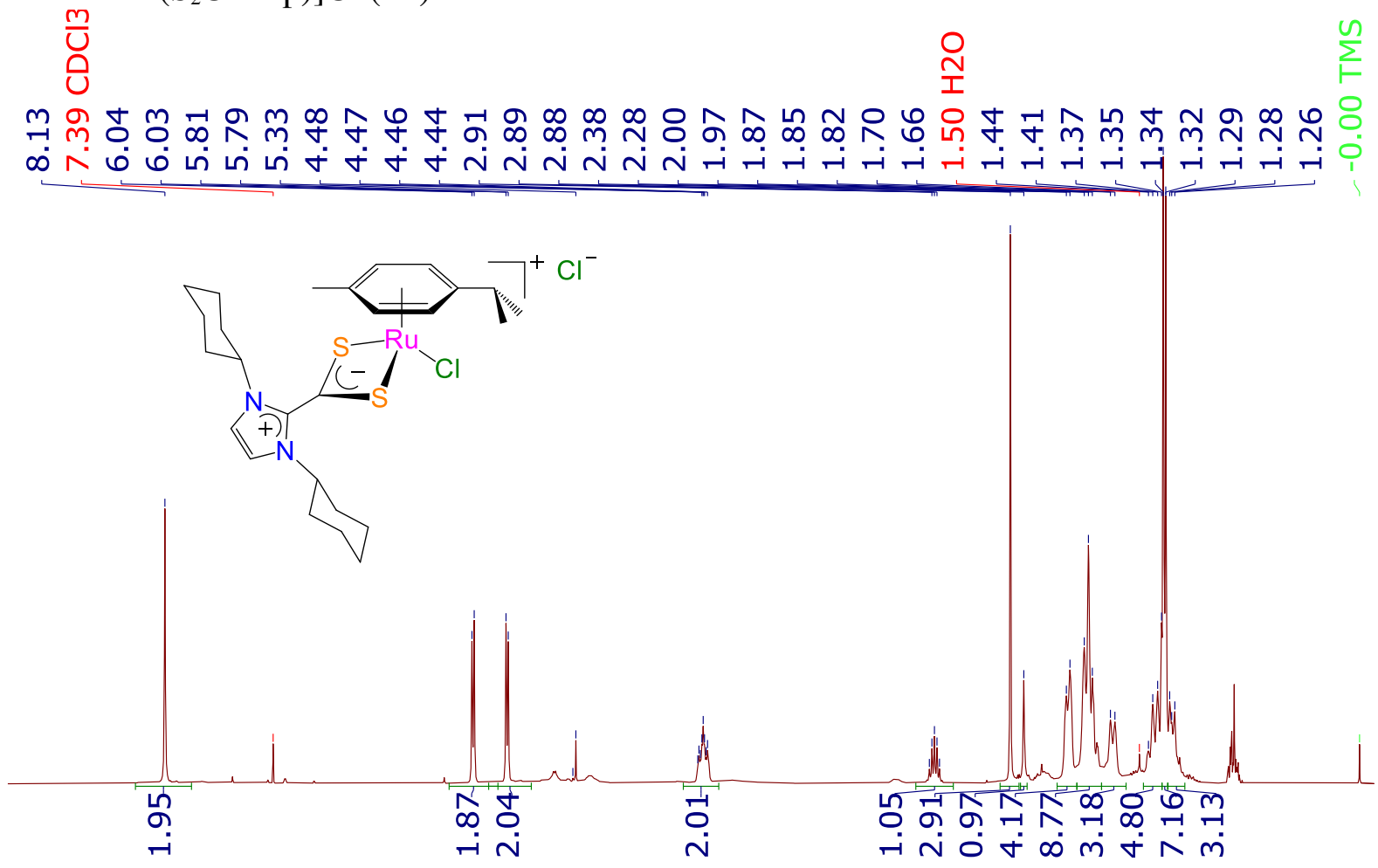

$\begin{array}{llllllllllllllllllllll}9.0 & 8.5 & 8.0 & 7.5 & 7.0 & 6.5 & 6.0 & 5.5 & 5.0 & 4.5 & 4.0 & 3.5 & 3.0 & 2.5 & 2.0 & 1.5 & 1.0 & 0.5 & 0.1\end{array}$ $\delta(\mathrm{ppm})$

Fig. S9. ${ }^{1} \mathrm{H}$ NMR spectrum $\left(400 \mathrm{MHz}, \mathrm{CDCl}_{3}, 298 \mathrm{~K}\right)$ of $\left[\mathrm{RuCl}(p\right.$-cymene $\left.)\left(\mathrm{S}_{2} \mathrm{C} \cdot \mathrm{ICy}\right)\right] \mathrm{Cl}$ $(\mathbf{2 c})$
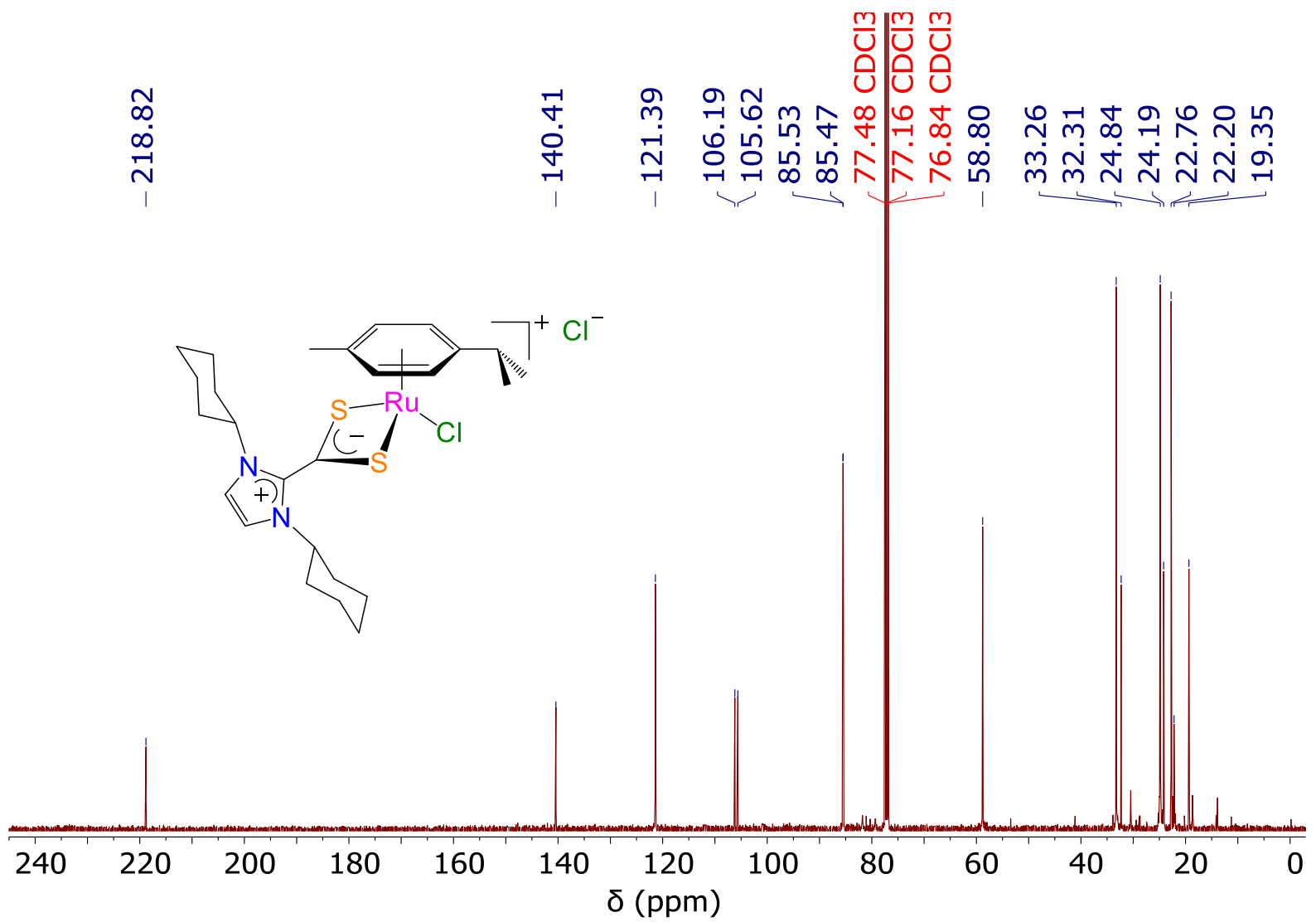
Fig. S10. ${ }^{13} \mathrm{C}\left\{{ }^{1} \mathrm{H}\right\} \mathrm{NMR}$ spectrum $\left(101 \mathrm{MHz}, \mathrm{CDCl}_{3}, 298 \mathrm{~K}\right)$ of $[\mathrm{RuCl}(p$-cymene)$\left.\left(\mathrm{S}_{2} \mathrm{C} \cdot \mathrm{ICy}\right)\right] \mathrm{Cl}(\mathbf{2 c})$

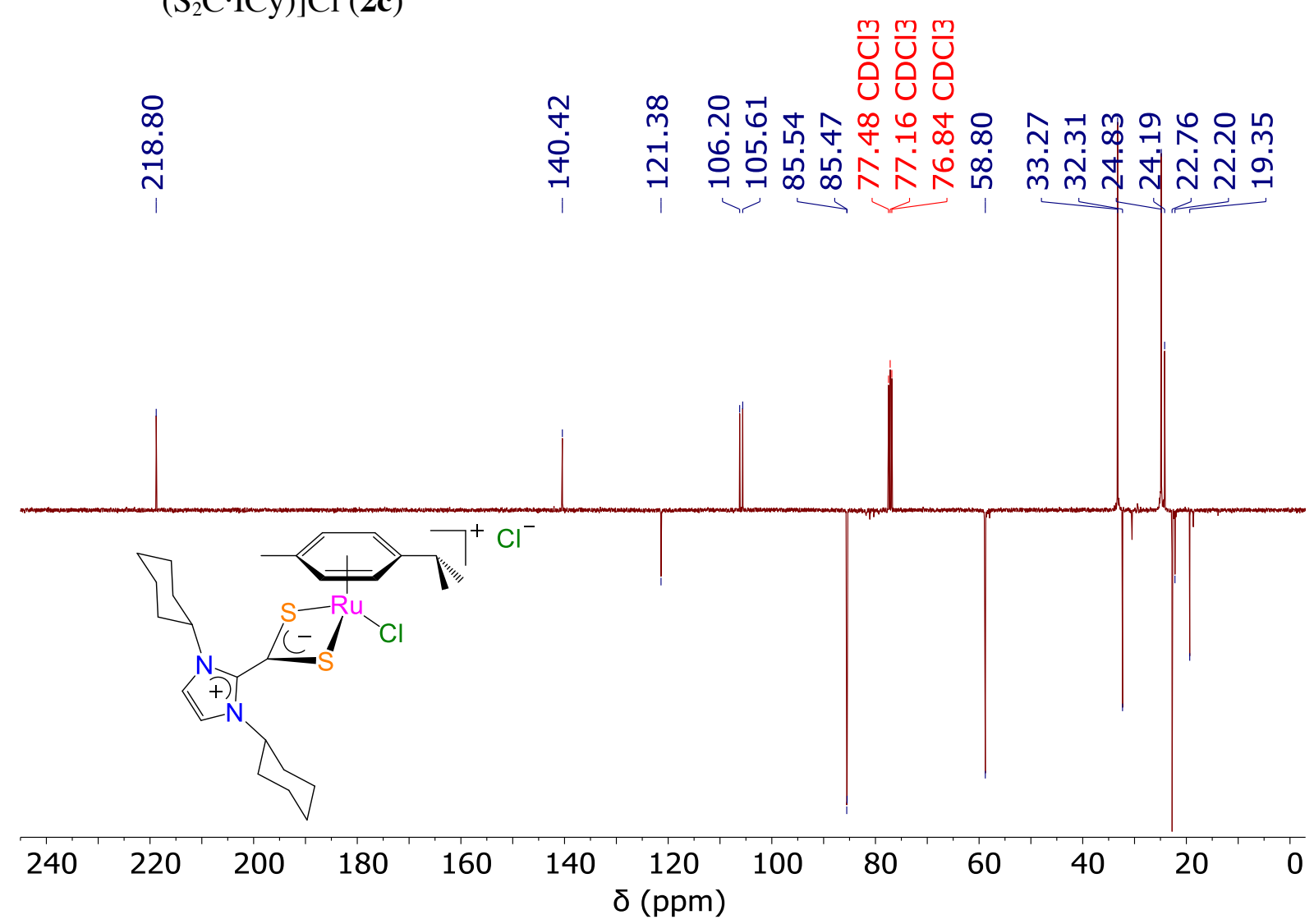

Fig. S11. ${ }^{13} \mathrm{C}\left\{{ }^{1} \mathrm{H}\right\}$ APT NMR spectrum $\left(101 \mathrm{MHz}, \mathrm{CDCl}_{3}, 298 \mathrm{~K}\right)$ of $[\mathrm{RuCl}(p$-cymene $)$ $\left.\left(\mathrm{S}_{2} \mathrm{C} \cdot \mathrm{ICy}\right)\right] \mathrm{Cl}(\mathbf{2 c})$

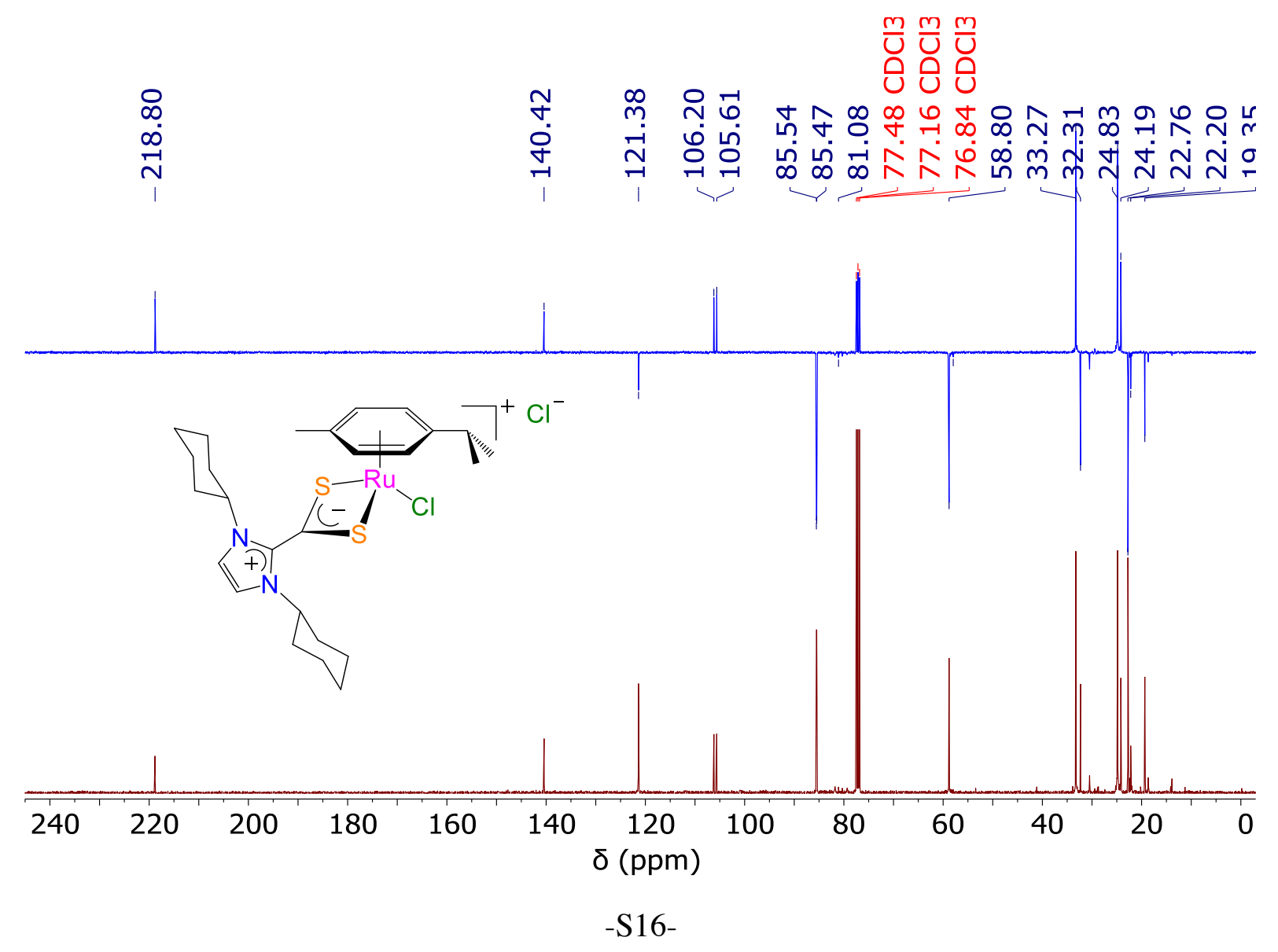


Fig. S12. ${ }^{13} \mathrm{C} \mathrm{CPD}$ and APT NMR spectra (101 $\left.\mathrm{MHz}, \mathrm{CDCl}_{3}, 298 \mathrm{~K}\right)$ of $[\mathrm{RuCl}(p$-cymene)-
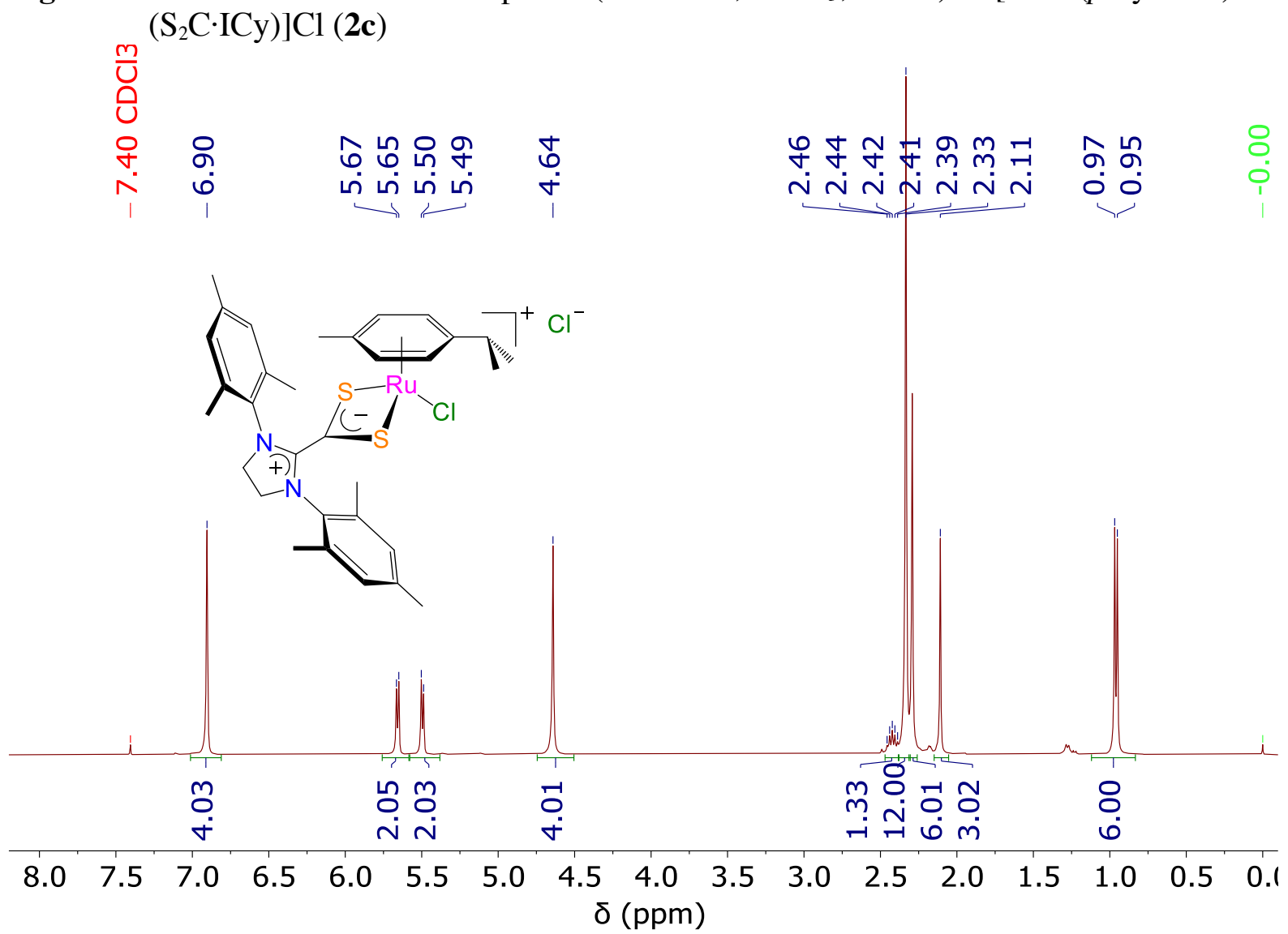

Fig. S13. ${ }^{1} \mathrm{H}$ NMR spectrum $\left(400 \mathrm{MHz}, \mathrm{CDCl}_{3}, 298 \mathrm{~K}\right)$ of $\left[\mathrm{RuCl}(p\right.$-cymene $\left.)\left(\mathrm{S}_{2} \mathrm{C} \cdot \mathrm{SIMes}\right)\right] \mathrm{Cl}$ (2d)
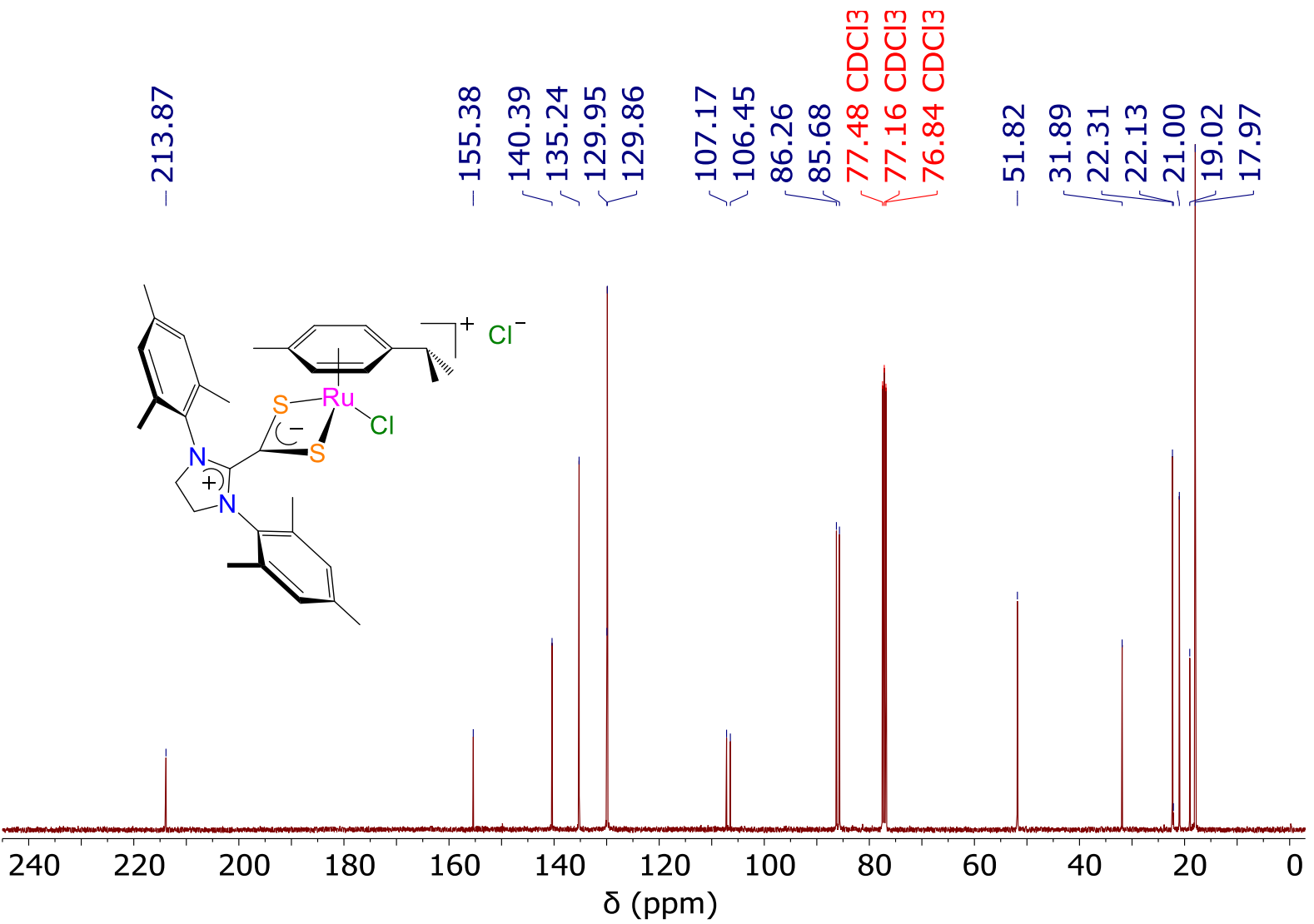
Fig. S14. ${ }^{13} \mathrm{C}\left\{{ }^{1} \mathrm{H}\right\}$ NMR spectrum $\left(101 \mathrm{MHz}, \mathrm{CDCl}_{3}, 298 \mathrm{~K}\right)$ of $[\mathrm{RuCl}(p$-cymene)$\left.\left(\mathrm{S}_{2} \mathrm{C} \cdot \mathrm{SIMes}\right)\right] \mathrm{Cl}(\mathbf{2 d})$

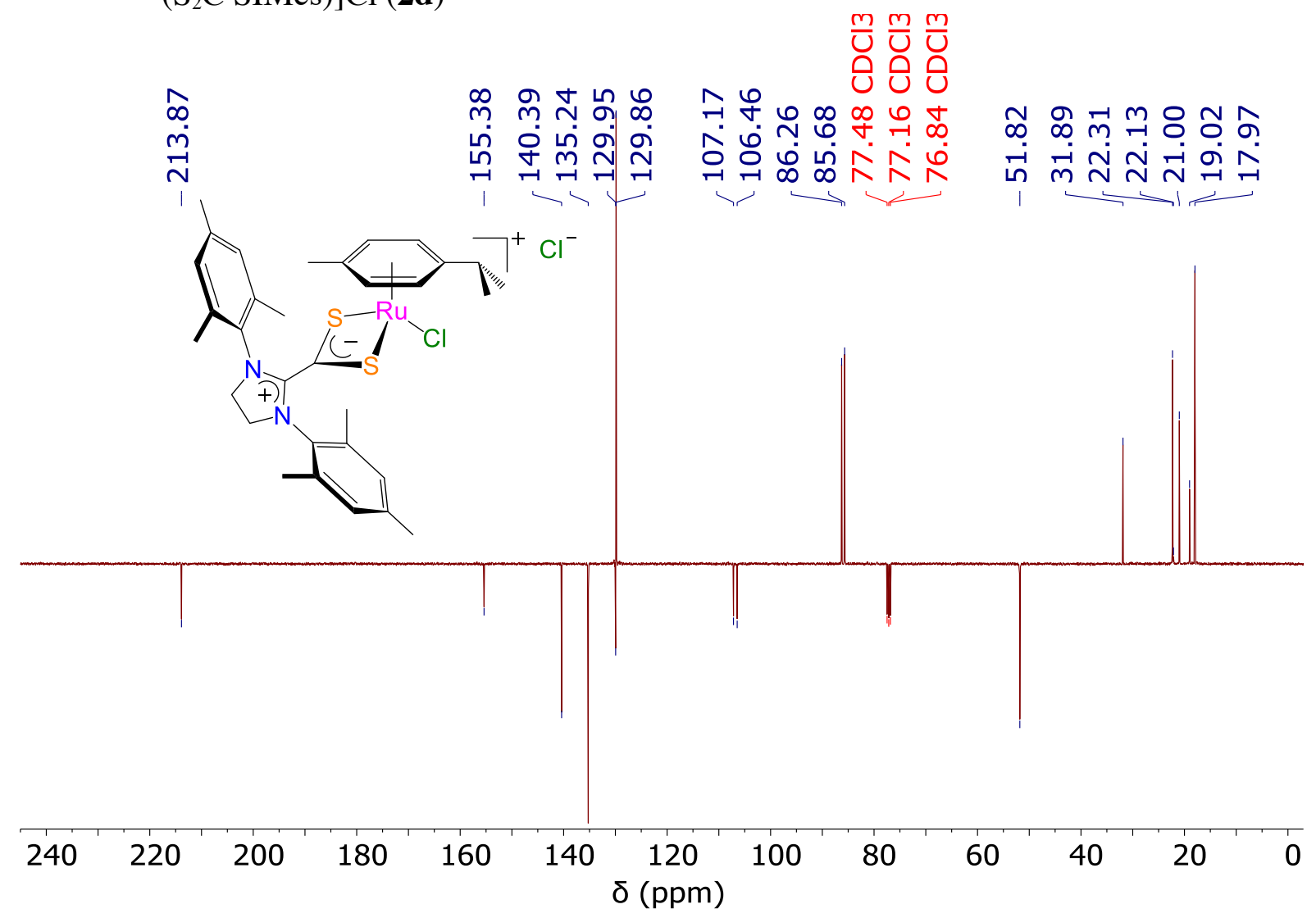

Fig. S15. ${ }^{13} \mathrm{C}\left\{{ }^{1} \mathrm{H}\right\}$ APT NMR spectrum $\left(101 \mathrm{MHz}, \mathrm{CDCl}_{3}, 298 \mathrm{~K}\right)$ of $[\mathrm{RuCl}(p$-cymene $)$ $\left.\left(\mathrm{S}_{2} \mathrm{C} \cdot \mathrm{SIMes}\right)\right] \mathrm{Cl}(\mathbf{2 d})$

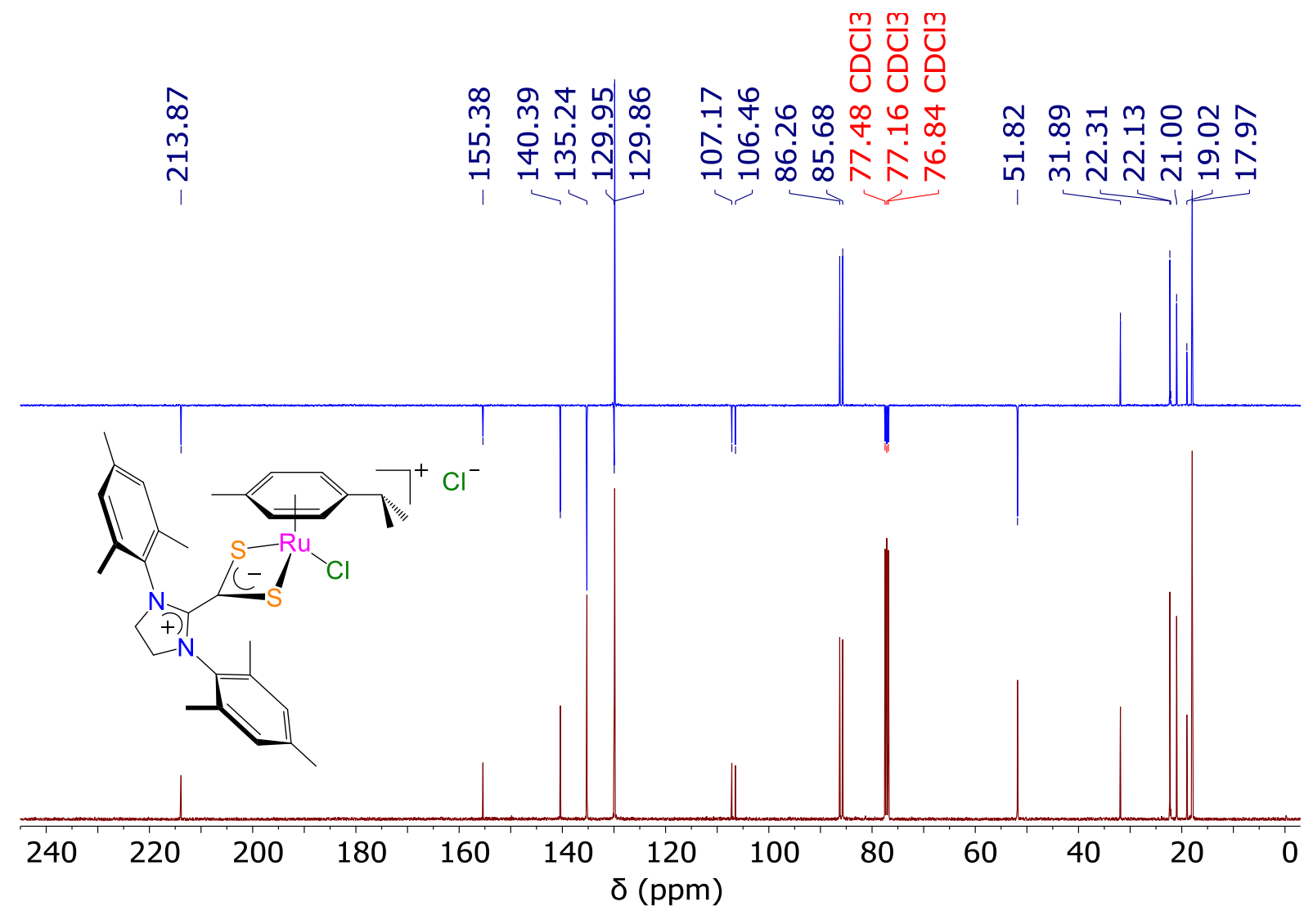


Fig. S16. ${ }^{13} \mathrm{C} \mathrm{CPD}$ and APT NMR spectra (101 $\left.\mathrm{MHz}, \mathrm{CDCl}_{3}, 298 \mathrm{~K}\right)$ of $[\mathrm{RuCl}(p$-cymene)$\left.\left(\mathrm{S}_{2} \mathrm{C} \cdot \mathrm{SIMes}\right)\right] \mathrm{Cl}$ (2d)<smiles>O[GeH2]</smiles>

윰 守 রূñ

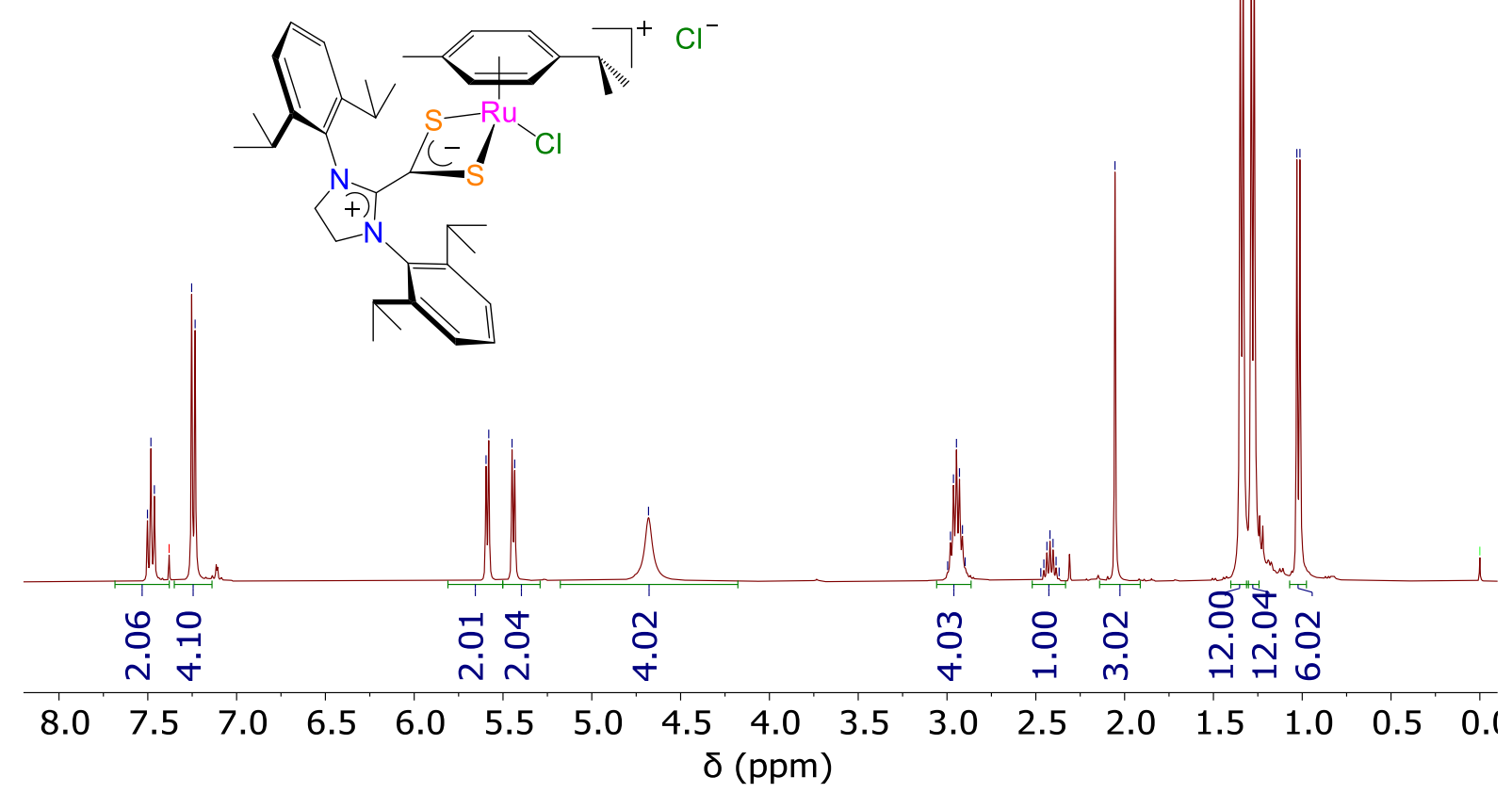

Fig. S17. ${ }^{1} \mathrm{H} \mathrm{NMR}$ spectrum $\left(400 \mathrm{MHz}, \mathrm{CDCl}_{3}, 298 \mathrm{~K}\right)$ of $\left[\mathrm{RuCl}(p\right.$-cymene $\left.)\left(\mathrm{S}_{2} \mathrm{C} \cdot \mathrm{SIDip}\right)\right] \mathrm{Cl}$ (2e)

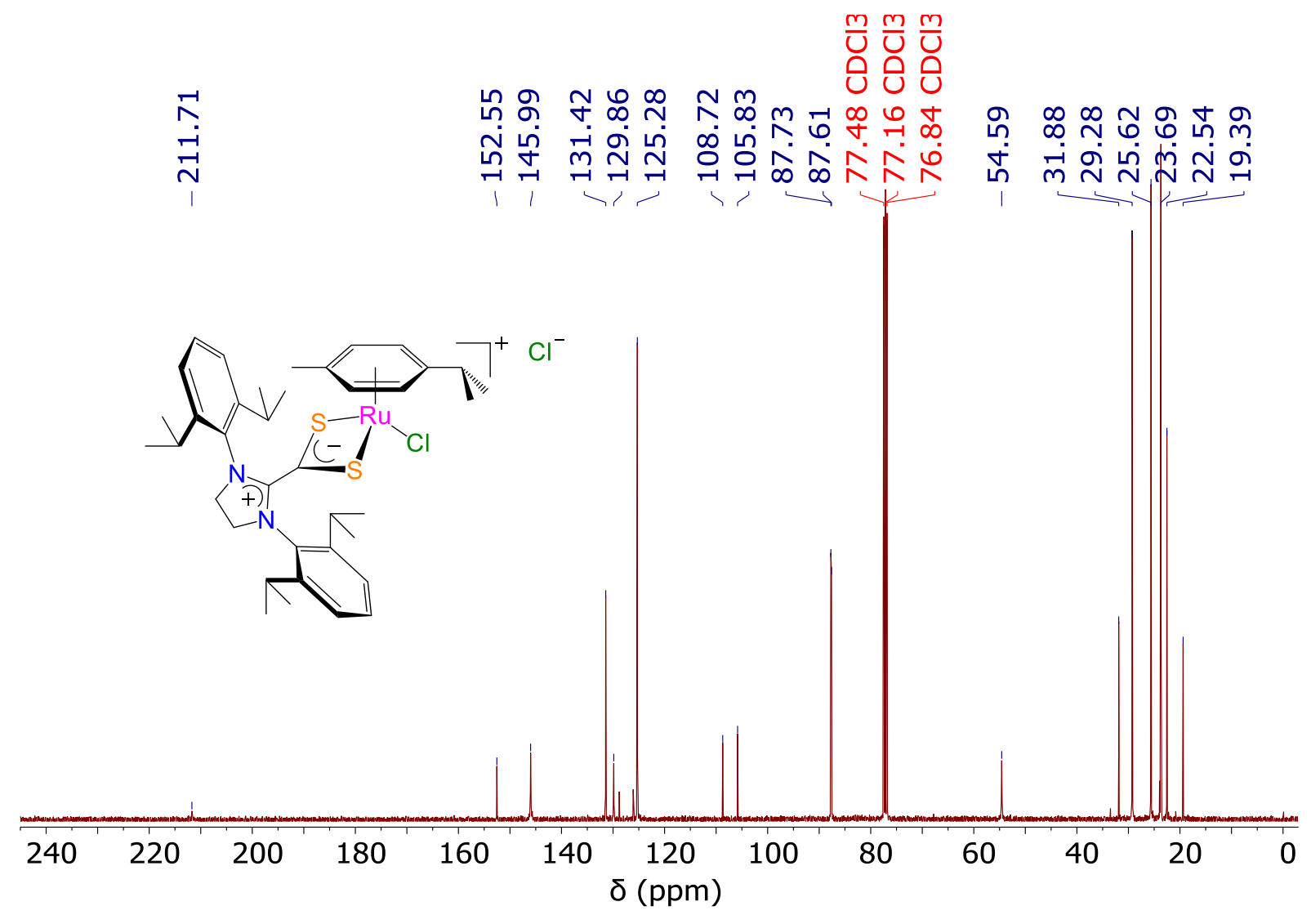


Fig. S18. ${ }^{13} \mathrm{C}\left\{{ }^{1} \mathrm{H}\right\}$ NMR spectrum $\left(101 \mathrm{MHz}, \mathrm{CDCl}_{3}, 298 \mathrm{~K}\right)$ of $[\mathrm{RuCl}(p$-cymene)$\left.\left(\mathrm{S}_{2} \mathrm{C} \cdot \mathrm{SIDip}\right)\right] \mathrm{Cl}(\mathbf{2 e})$

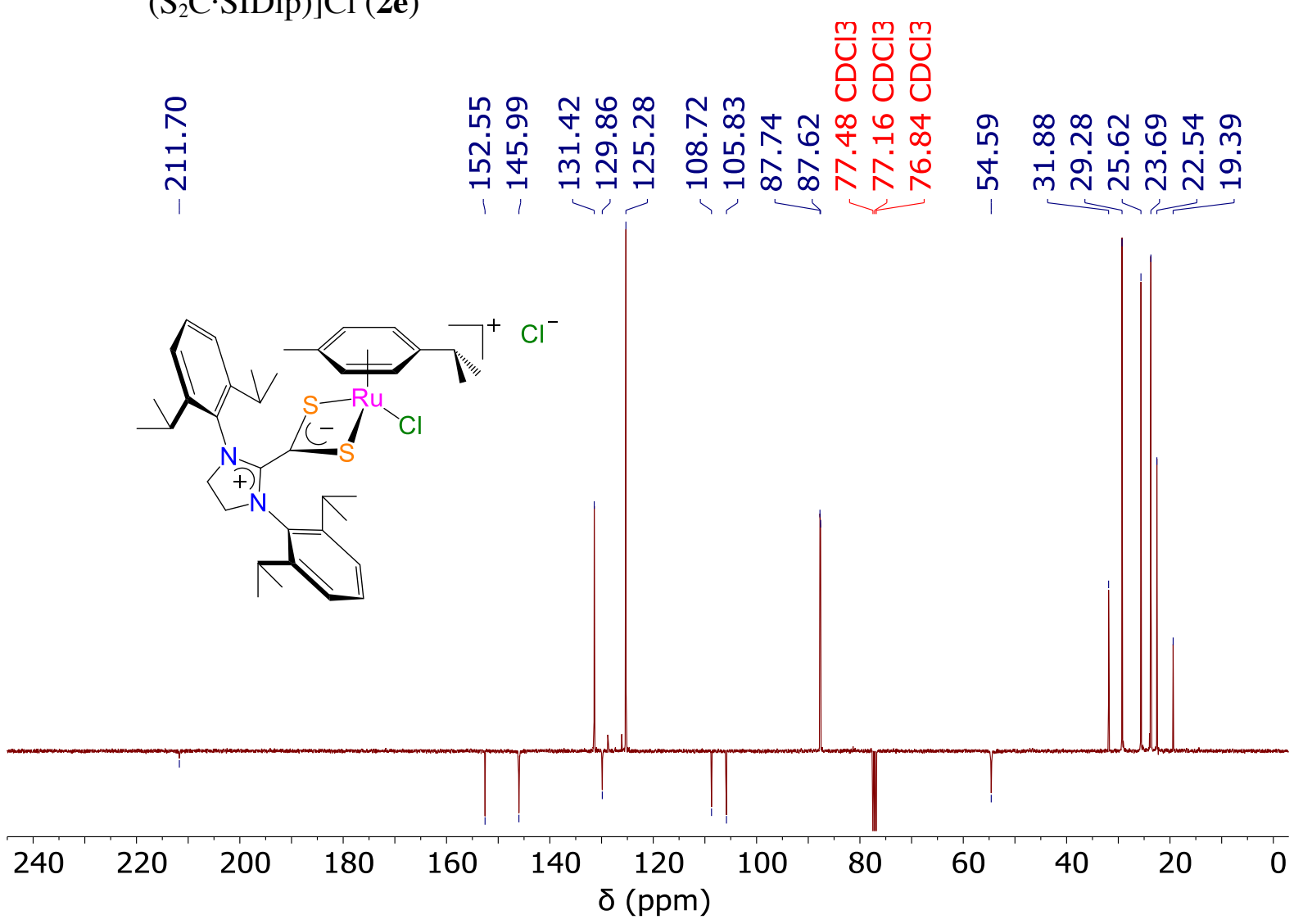

Fig. S19. ${ }^{13} \mathrm{C}\left\{{ }^{1} \mathrm{H}\right\}$ APT NMR spectrum $\left(101 \mathrm{MHz}, \mathrm{CDCl}_{3}, 298 \mathrm{~K}\right)$ of $[\mathrm{RuCl}(p$-cymene $)$ $\left.\left(\mathrm{S}_{2} \mathrm{C} \cdot \mathrm{SIDip}\right)\right] \mathrm{Cl}(\mathbf{2 e})$

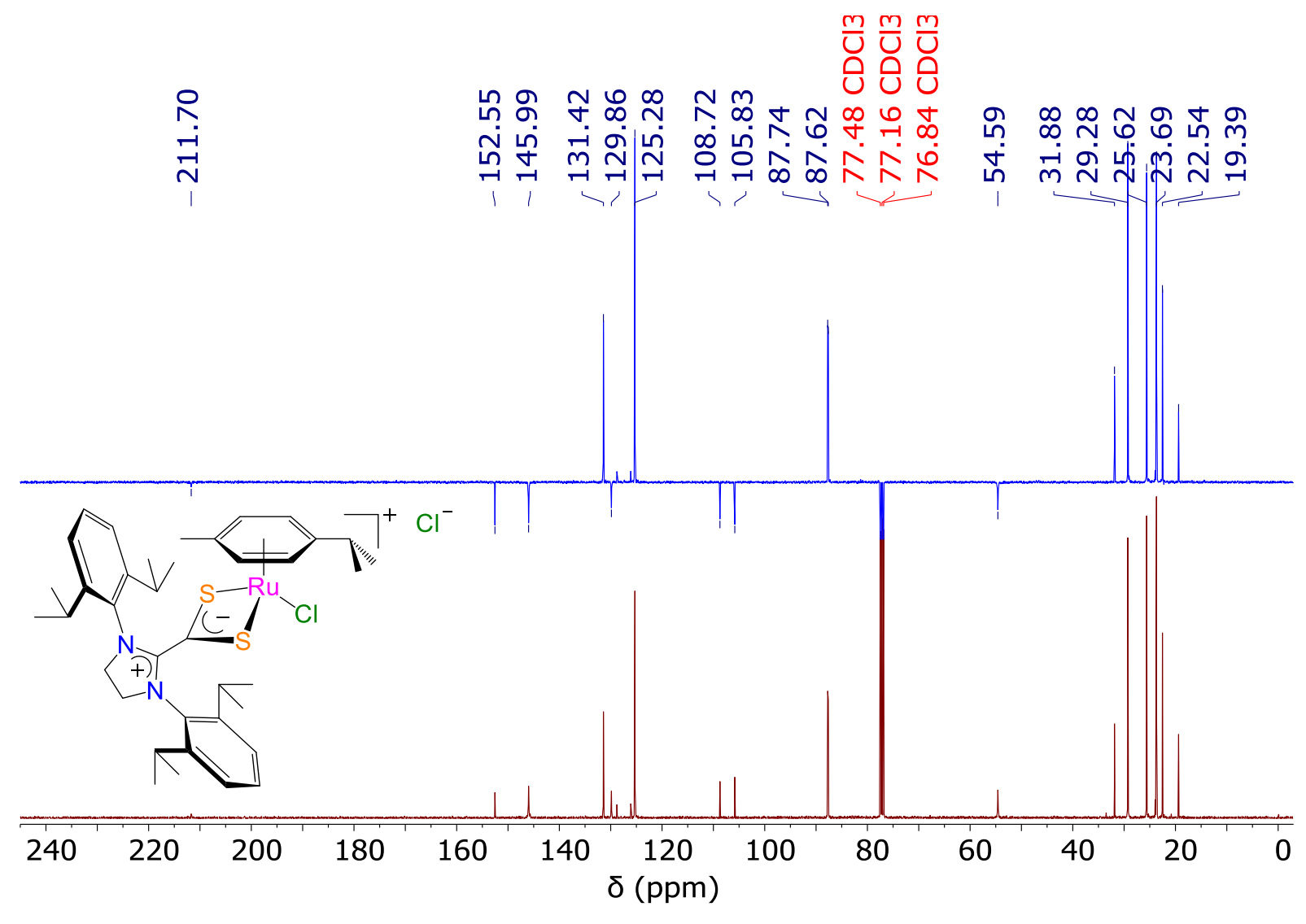


Fig. S20. ${ }^{13} \mathrm{C} \mathrm{CPD}$ and APT NMR spectra $\left(101 \mathrm{MHz}, \mathrm{CDCl}_{3}, 298 \mathrm{~K}\right)$ of $[\mathrm{RuCl}(p$-cymene $)-$ $\left.\left(\mathrm{S}_{2} \mathrm{C} \cdot \mathrm{SIDip}\right)\right] \mathrm{Cl}(\mathbf{2 e})$<smiles></smiles>

夰 $\infty$ ¿ $^{\circ}$

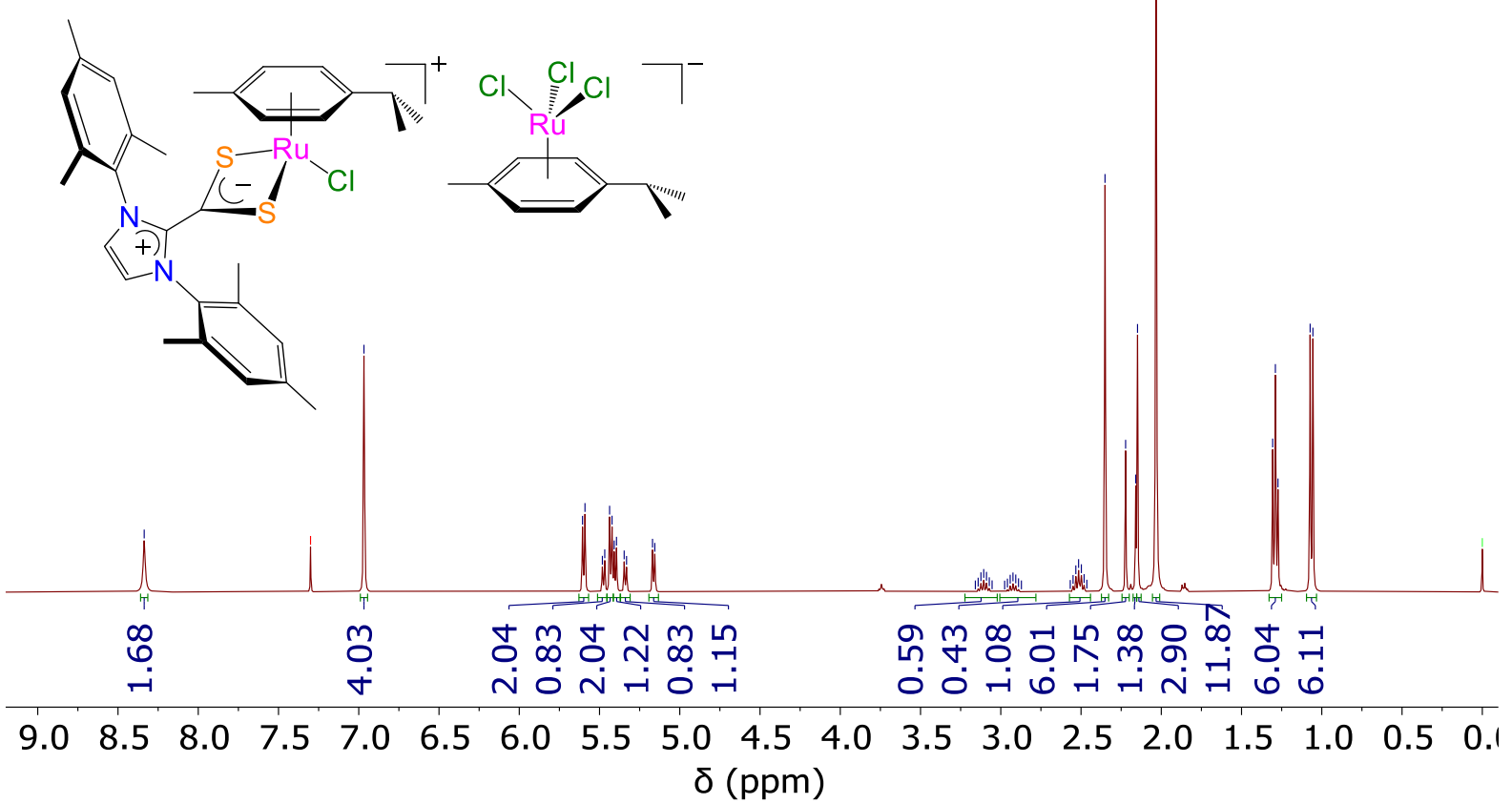

Fig. S21. ${ }^{1} \mathrm{H}$ NMR spectrum $\left(400 \mathrm{MHz}, \mathrm{CDCl}_{3}, 298 \mathrm{~K}\right)$ of $\left[\mathrm{RuCl}(p\right.$-cymene $\left.)\left(\mathrm{S}_{2} \mathrm{C} \cdot \mathrm{IMes}\right)\right]-$ $\left[\mathrm{RuCl}_{3}(p\right.$-cymene) $)(\mathbf{3 a})$

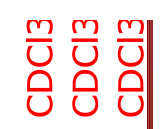

근 뉴ํ

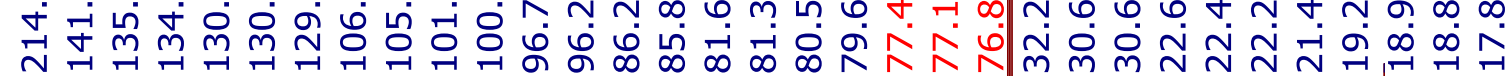

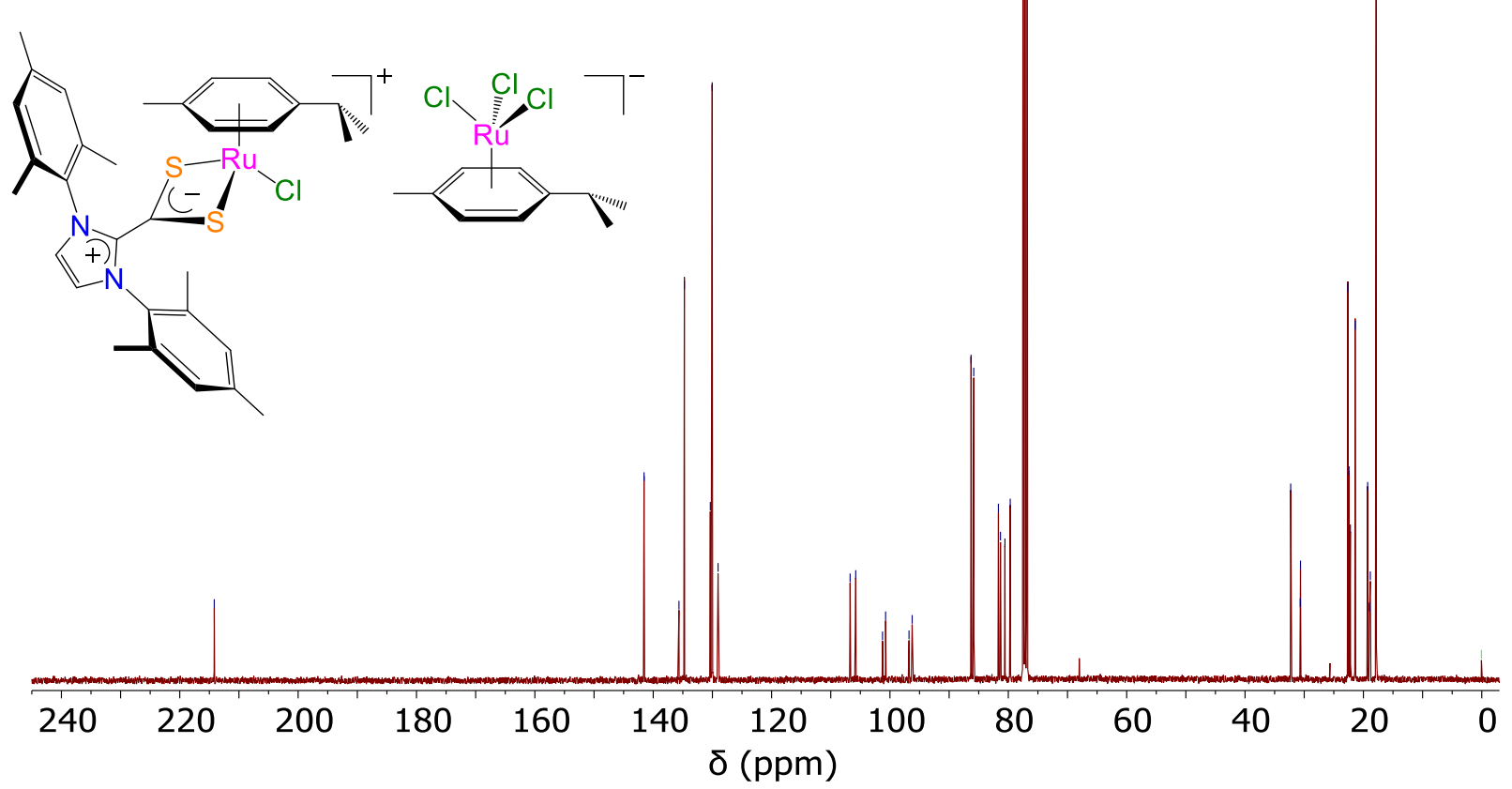


Fig. S22. ${ }^{13} \mathrm{C}\left\{{ }^{1} \mathrm{H}\right\}$ NMR spectrum $\left(101 \mathrm{MHz}, \mathrm{CDCl}_{3}, 298 \mathrm{~K}\right)$ of $[\mathrm{RuCl}(p$-cymene $)$ $\left.\left(\mathrm{S}_{2} \mathrm{C} \cdot \mathrm{IMes}\right)\right]\left[\mathrm{RuCl}_{3}(p\right.$-cymene $\left.)\right](\mathbf{3 a})$

근

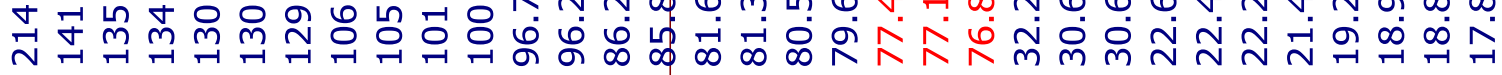

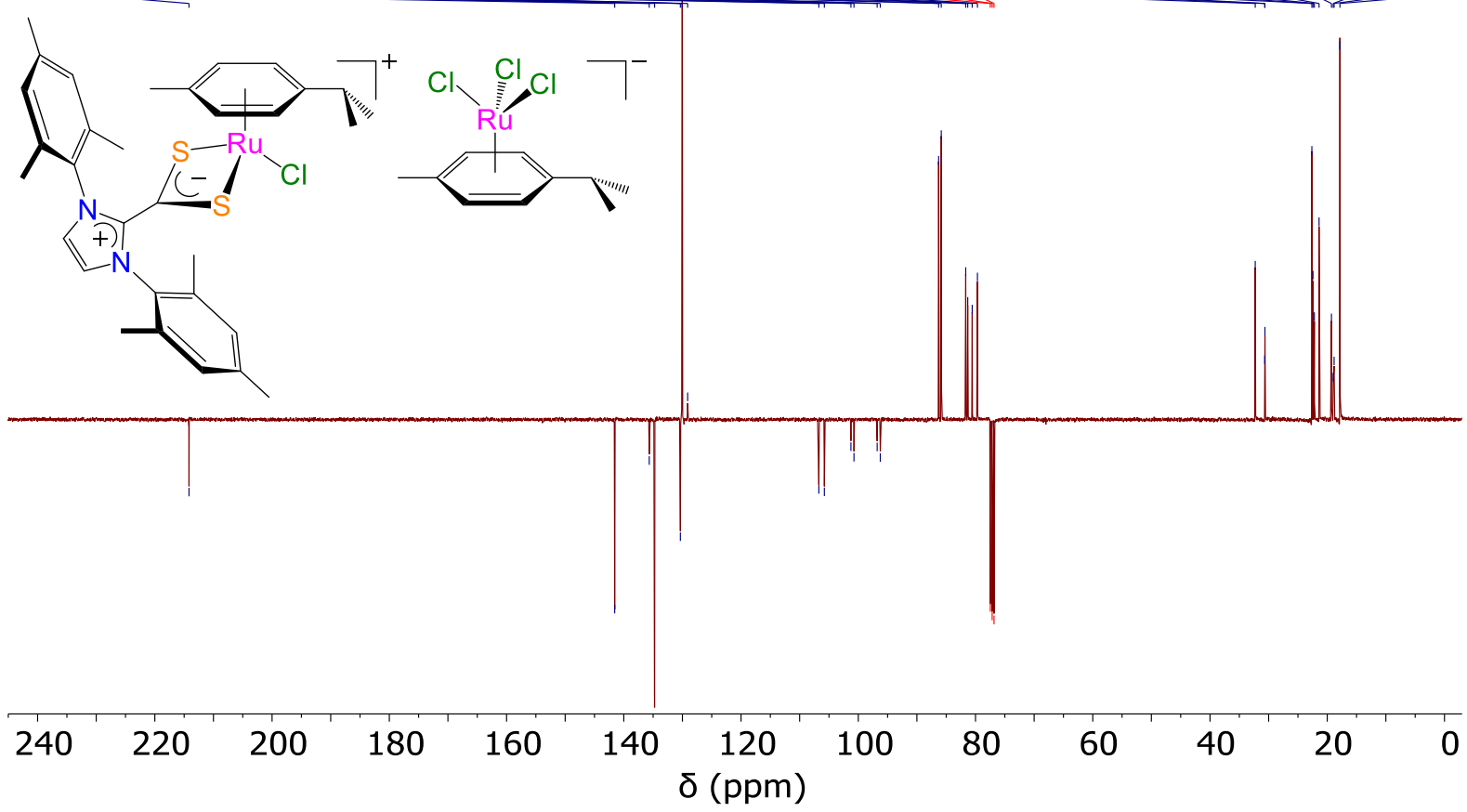

Fig. S23. ${ }^{13} \mathrm{C}\left\{{ }^{1} \mathrm{H}\right\}$ APT NMR spectrum $\left(101 \mathrm{MHz}, \mathrm{CDCl}_{3}, 298 \mathrm{~K}\right)$ of $[\mathrm{RuCl}(p$-cymene $)$ $\left.\left(\mathrm{S}_{2} \mathrm{C} \cdot \mathrm{IMes}\right)\right]\left[\mathrm{RuCl}_{3}(p\right.$-cymene $\left.)\right](\mathbf{3 a})$

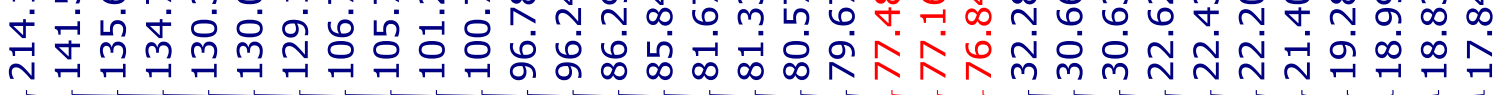

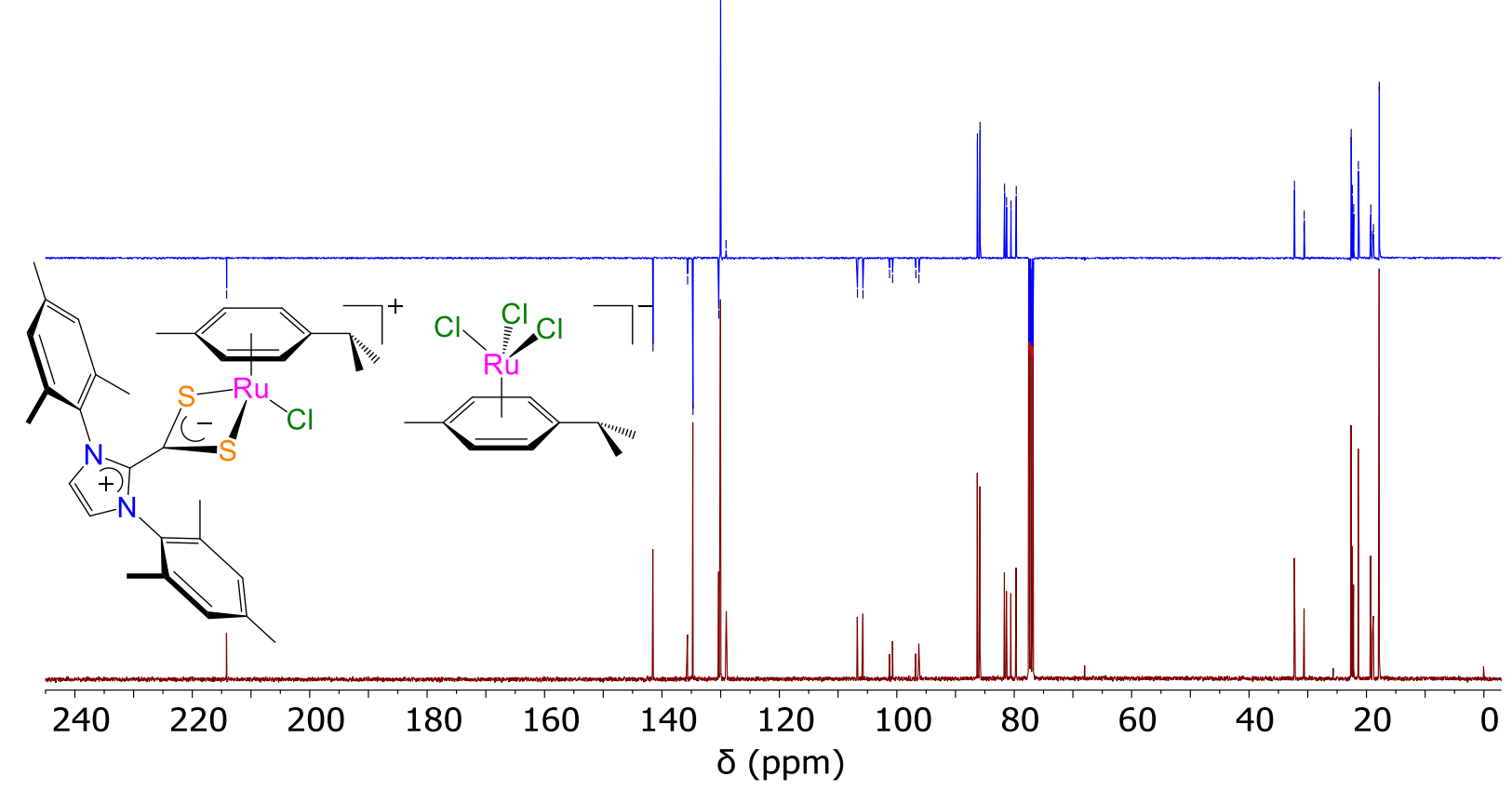


Fig. S24. ${ }^{13} \mathrm{C} \mathrm{CPD}$ and APT NMR spectra (101 $\left.\mathrm{MHz}, \mathrm{CDCl}_{3}, 298 \mathrm{~K}\right)$ of $[\mathrm{RuCl}(p$-cymene)$\left.\left(\mathrm{S}_{2} \mathrm{C} \cdot \mathrm{IMes}\right)\right]\left[\mathrm{RuCl}_{3}(\mathrm{p}\right.$-cymene $\left.)\right](\mathbf{3 a})$

q

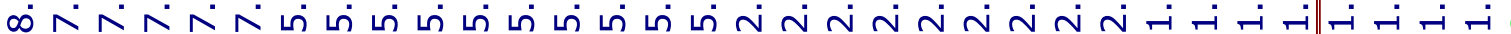

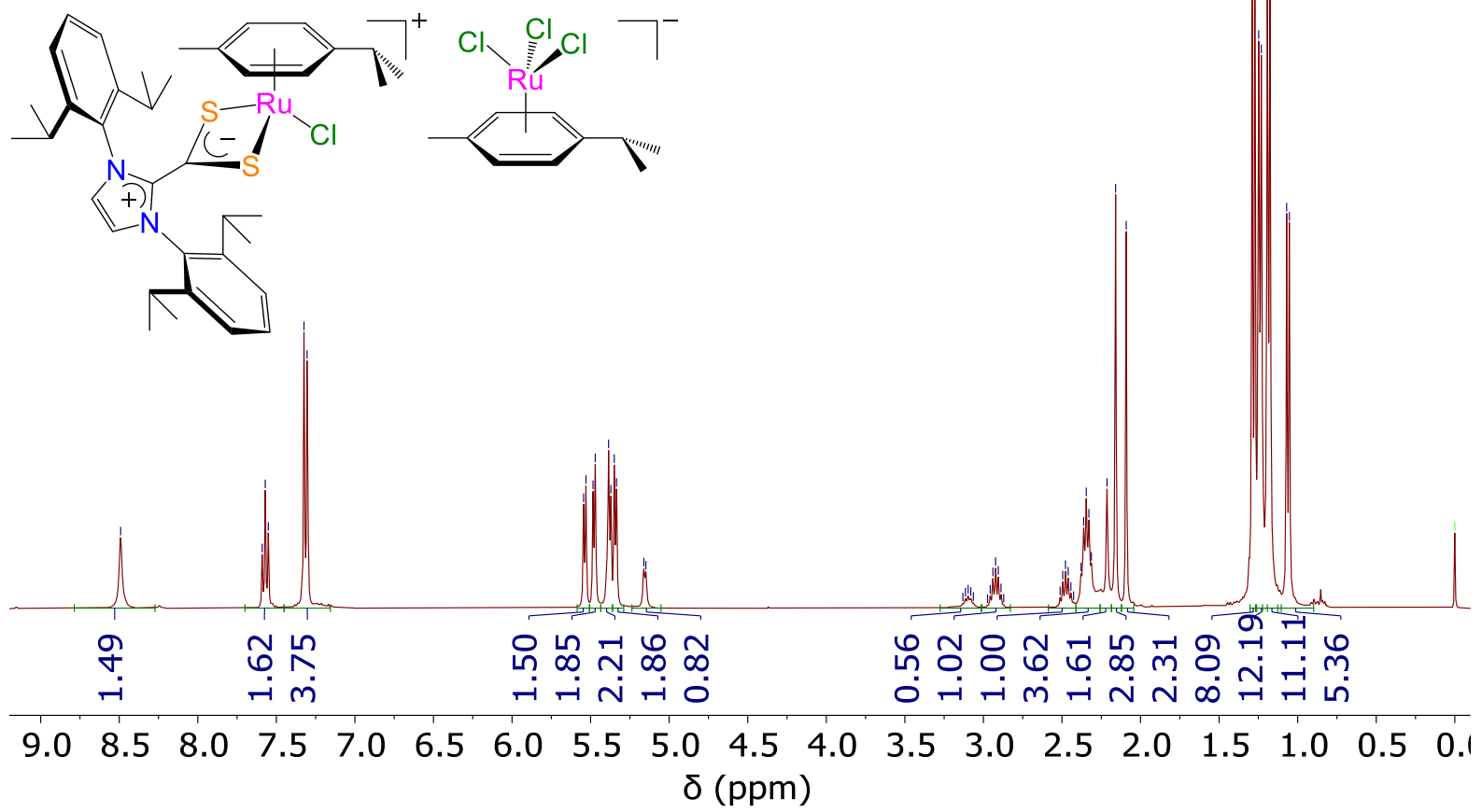

Fig. S25. ${ }^{1} \mathrm{H}$ NMR spectrum $\left(400 \mathrm{MHz}, \mathrm{CDCl}_{3}, 298 \mathrm{~K}\right)$ of $\left[\mathrm{RuCl}(p\right.$-cymene $\left.)\left(\mathrm{S}_{2} \mathrm{C} \cdot \mathrm{IDip}\right)\right]$ $\left[\mathrm{RuCl}_{3}(p\right.$-cymene $\left.)\right](\mathbf{3 b})$

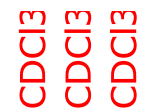

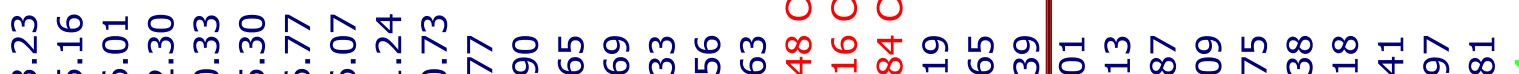
光
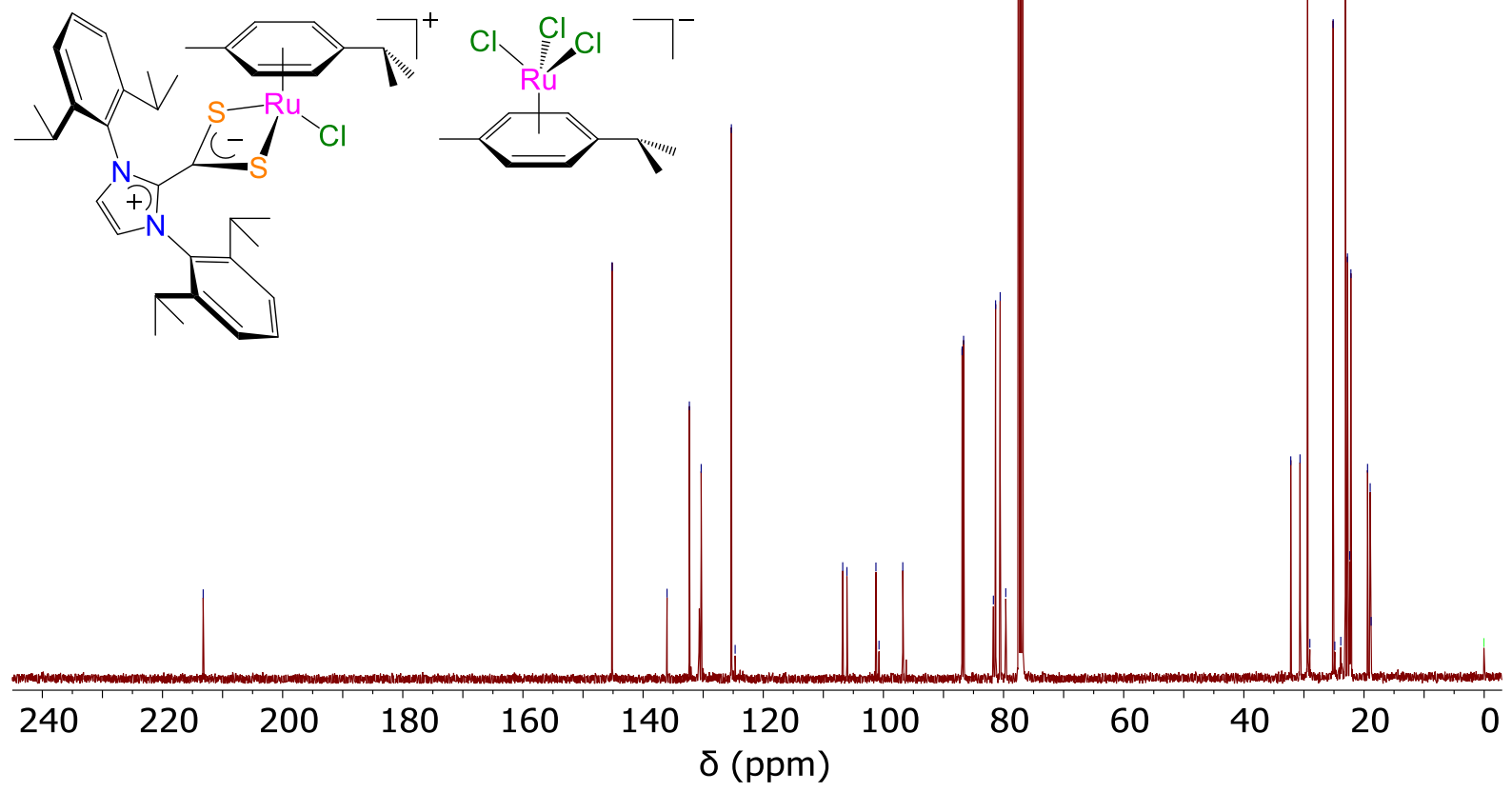
Fig. S26. ${ }^{13} \mathrm{C}\left\{{ }^{1} \mathrm{H}\right\} \mathrm{NMR}$ spectrum $\left(101 \mathrm{MHz}, \mathrm{CDCl}_{3}, 298 \mathrm{~K}\right)$ of $[\mathrm{RuCl}(p$-cymene)$\left.\left(\mathrm{S}_{2} \mathrm{C} \cdot \mathrm{IDip}\right)\right]\left[\mathrm{RuCl}_{3}(p\right.$-cymene $\left.)\right](\mathbf{3 b})$

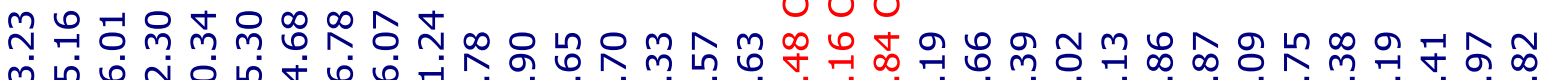
국

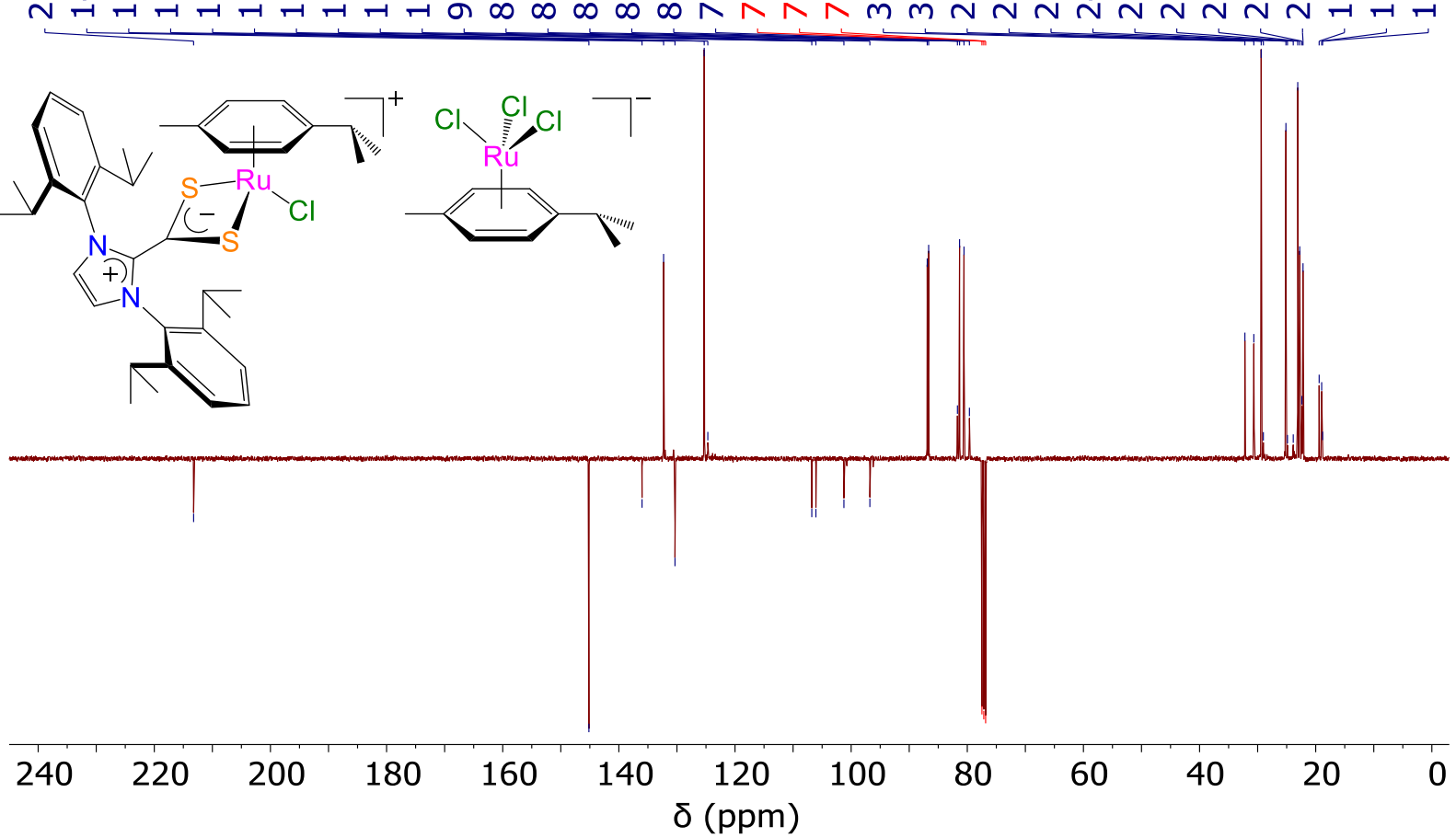

Fig. S27. ${ }^{13} \mathrm{C}\left\{{ }^{1} \mathrm{H}\right\}$ APT NMR spectrum $\left(101 \mathrm{MHz}, \mathrm{CDCl}_{3}, 298 \mathrm{~K}\right)$ of $[\mathrm{RuCl}(p$-cymene $)$ $\left.\left(\mathrm{S}_{2} \mathrm{C} \cdot \mathrm{IDip}\right)\right]\left[\mathrm{RuCl}_{3}(p\right.$-cymene $\left.)\right](3 \mathrm{~b})$

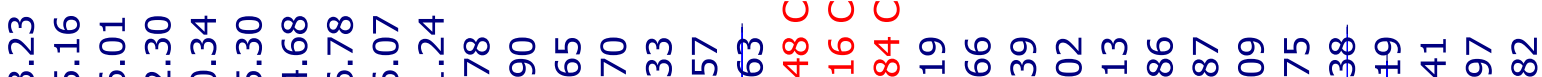
ஸे ம்

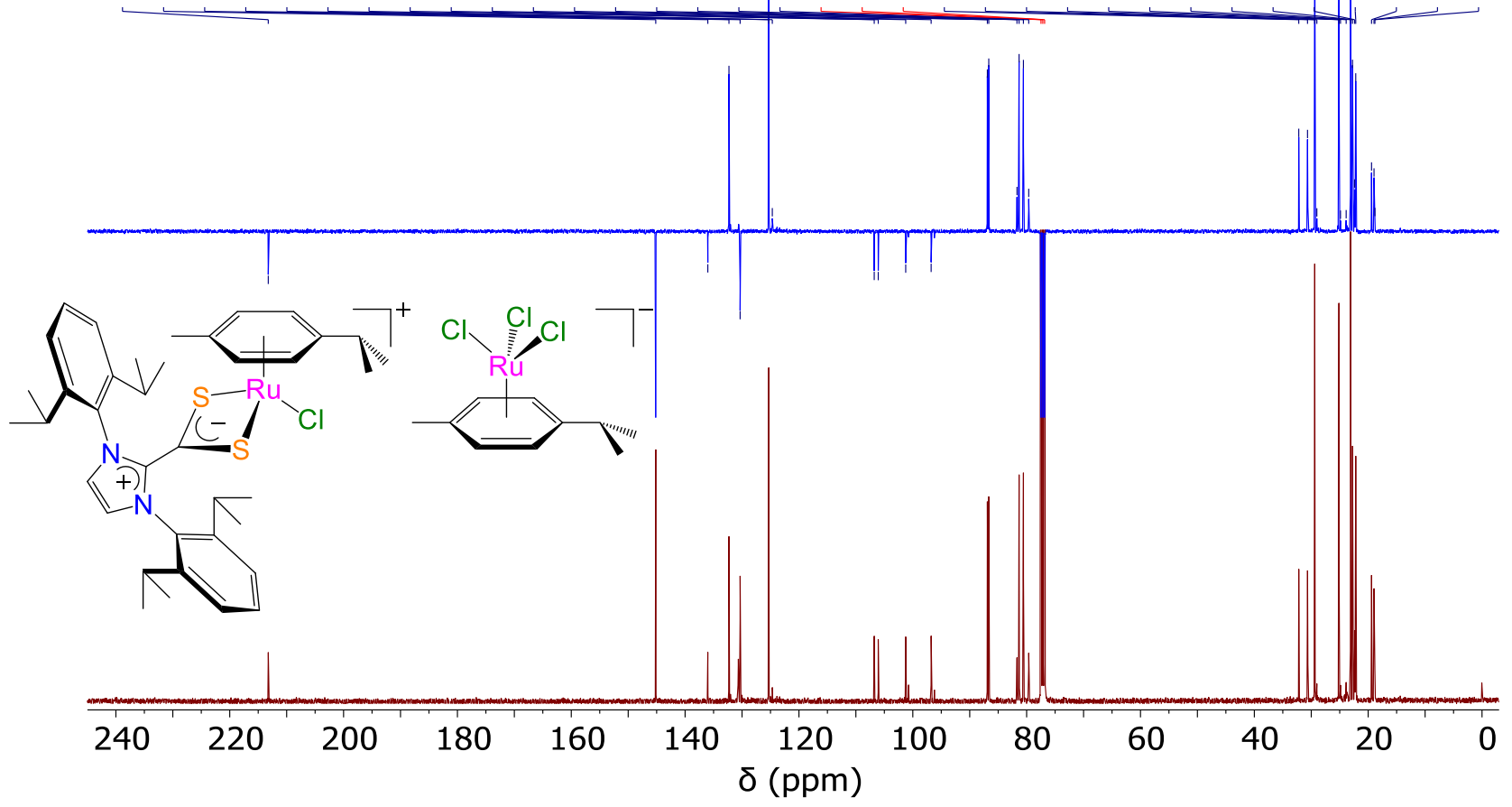


Fig. S28. ${ }^{13} \mathrm{C} \mathrm{CPD}$ and APT NMR spectra $\left(101 \mathrm{MHz}, \mathrm{CDCl}_{3}, 298 \mathrm{~K}\right)$ of $[\mathrm{RuCl}(p$-cymene $)-$ $\left.\left(\mathrm{S}_{2} \mathrm{C} \cdot \mathrm{IDip}\right)\right]\left[\mathrm{RuCl}_{3}(p\right.$-cymene $\left.)\right](\mathbf{3 b})$

บั

ન

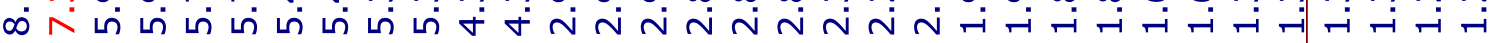

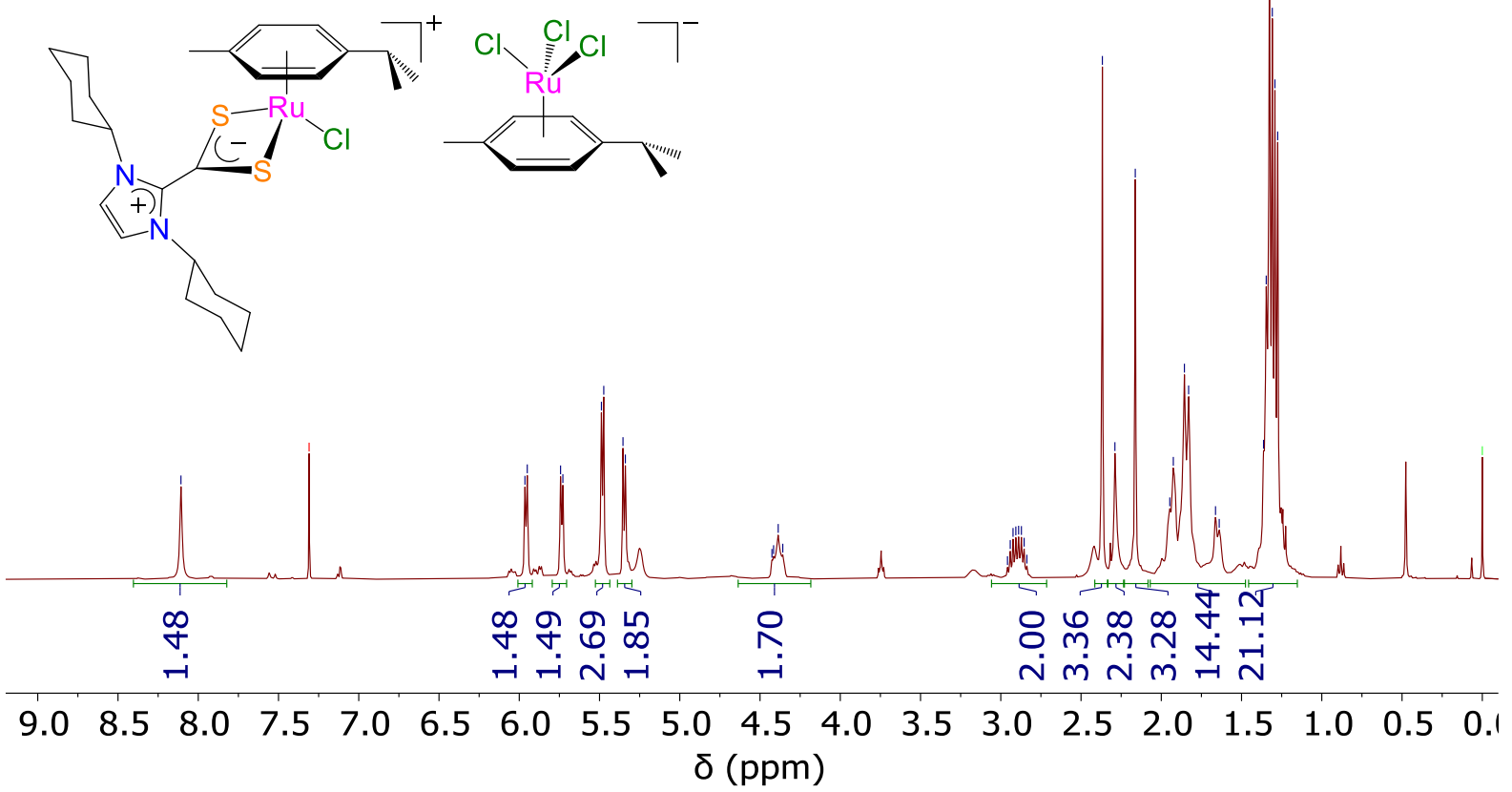

Fig. S29. ${ }^{1} \mathrm{H} \mathrm{NMR}$ spectrum $\left(400 \mathrm{MHz}, \mathrm{CDCl}_{3}, 298 \mathrm{~K}\right)$ of $\left[\mathrm{RuCl}(p\right.$-cymene $\left.)\left(\mathrm{S}_{2} \mathrm{C} \cdot \mathrm{ICy}\right)\right]-$ $\left[\mathrm{RuCl}_{3}(p\right.$-cymene $\left.)\right](\mathbf{3 c})$

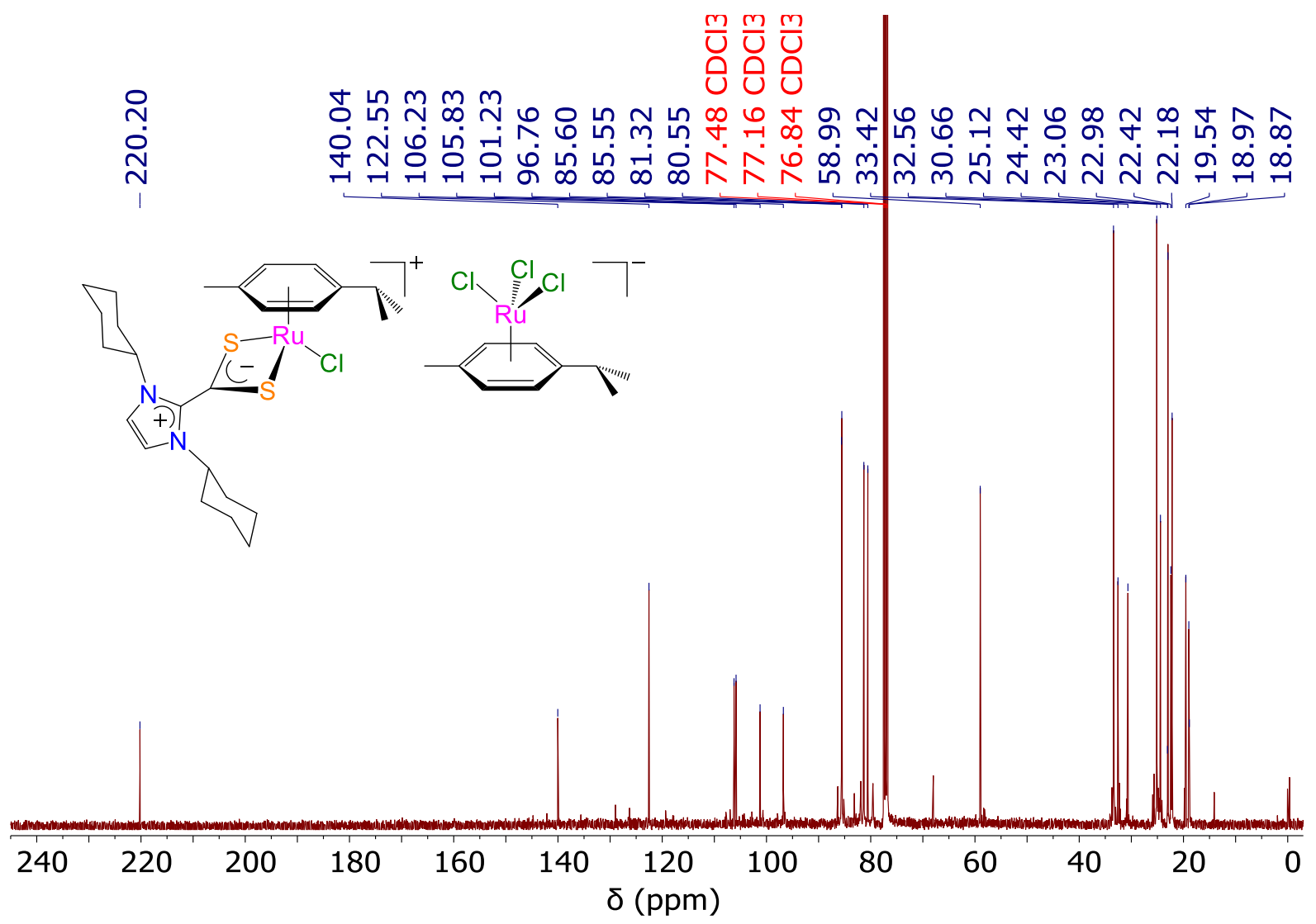


Fig. S30. ${ }^{13} \mathrm{C}\left\{{ }^{1} \mathrm{H}\right\}$ NMR spectrum $\left(101 \mathrm{MHz}, \mathrm{CDCl}_{3}, 298 \mathrm{~K}\right)$ of $\left[\mathrm{RuCl}(p\right.$-cymene $\left.)\left(\mathrm{S}_{2} \mathrm{C} \cdot \mathrm{ICy}\right)\right]-$ $\left[\mathrm{RuCl}_{3}(p\right.$-cymene $\left.)\right](\mathbf{3 c})$

굼 ํํำ

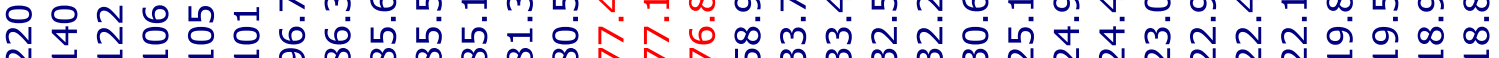

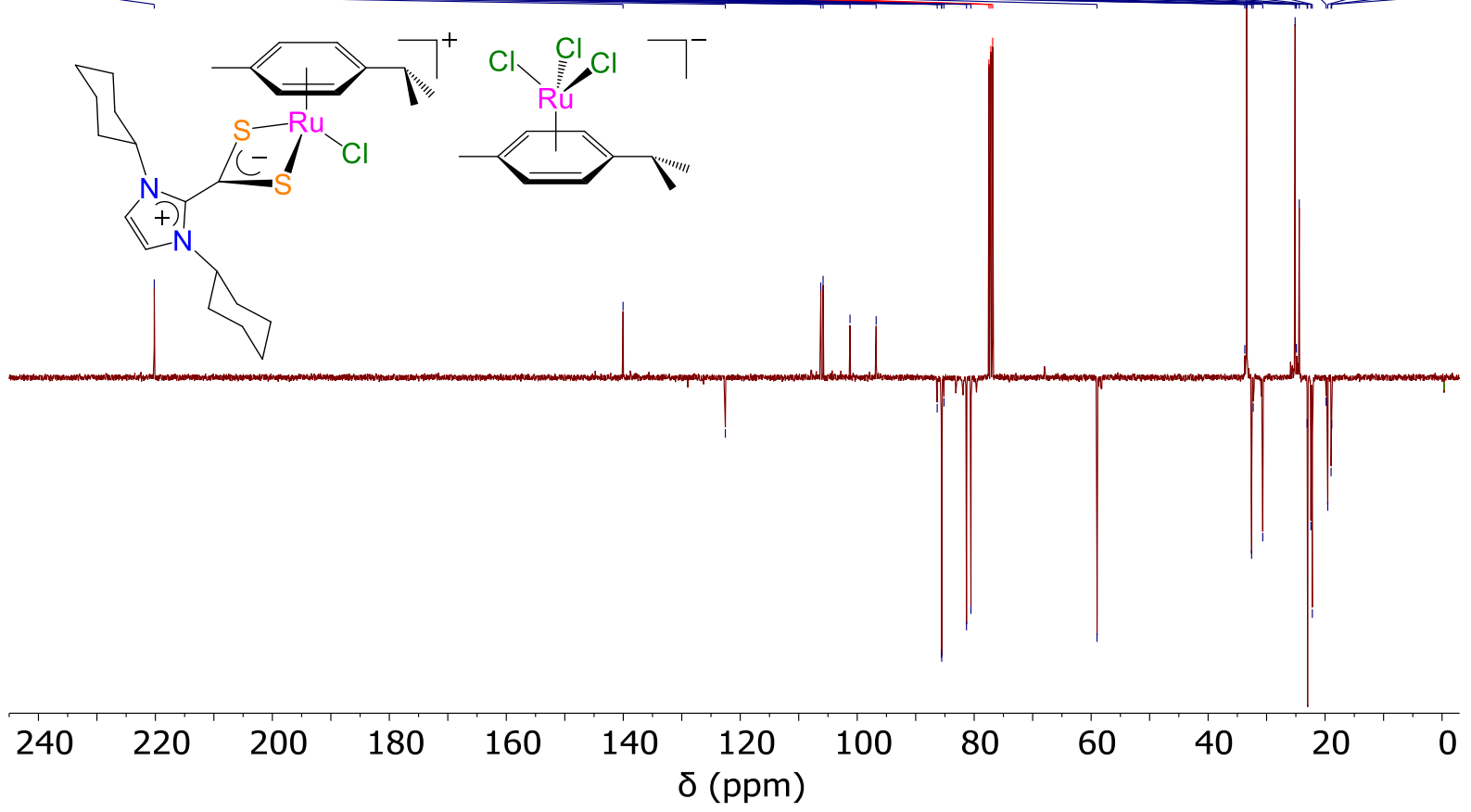

Fig. S31. ${ }^{13} \mathrm{C}\left\{{ }^{1} \mathrm{H}\right\}$ APT NMR spectrum $\left(101 \mathrm{MHz}, \mathrm{CDCl}_{3}, 298 \mathrm{~K}\right)$ of $[\mathrm{RuCl}(p$-cymene $)$ $\left.\left(\mathrm{S}_{2} \mathrm{C} \cdot \mathrm{ICy}\right)\right]\left[\mathrm{RuCl}_{3}(p\right.$-cymene $\left.)\right](3 \mathrm{c})$

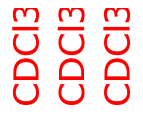

궁 ㄴํํ กิ

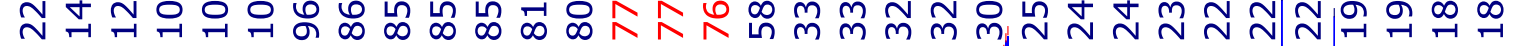

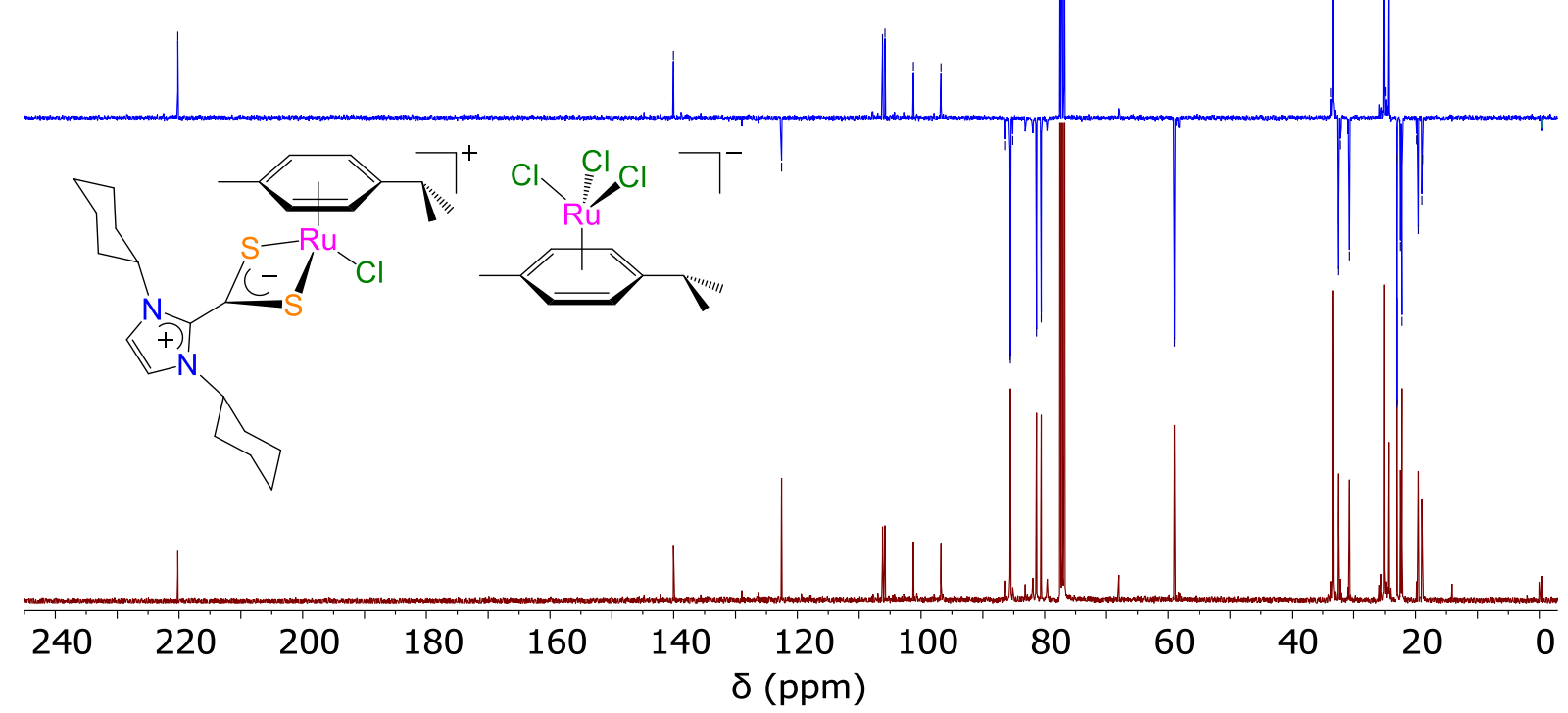


Fig. S32. ${ }^{13} \mathrm{C} \mathrm{CPD}$ and APT NMR spectra $\left(101 \mathrm{MHz}, \mathrm{CDCl}_{3}, 298 \mathrm{~K}\right)$ of $[\mathrm{RuCl}(p$-cymene $)-$ $\left.\left(\mathrm{S}_{2} \mathrm{C} \cdot \mathrm{ICy}\right)\right]\left[\mathrm{RuCl}_{3}(p\right.$-cymene) $](\mathbf{3 c})$

ํㅡㅁ

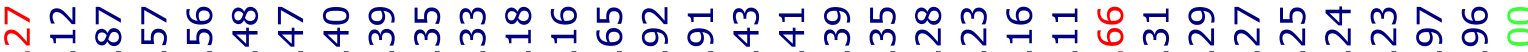
กล่

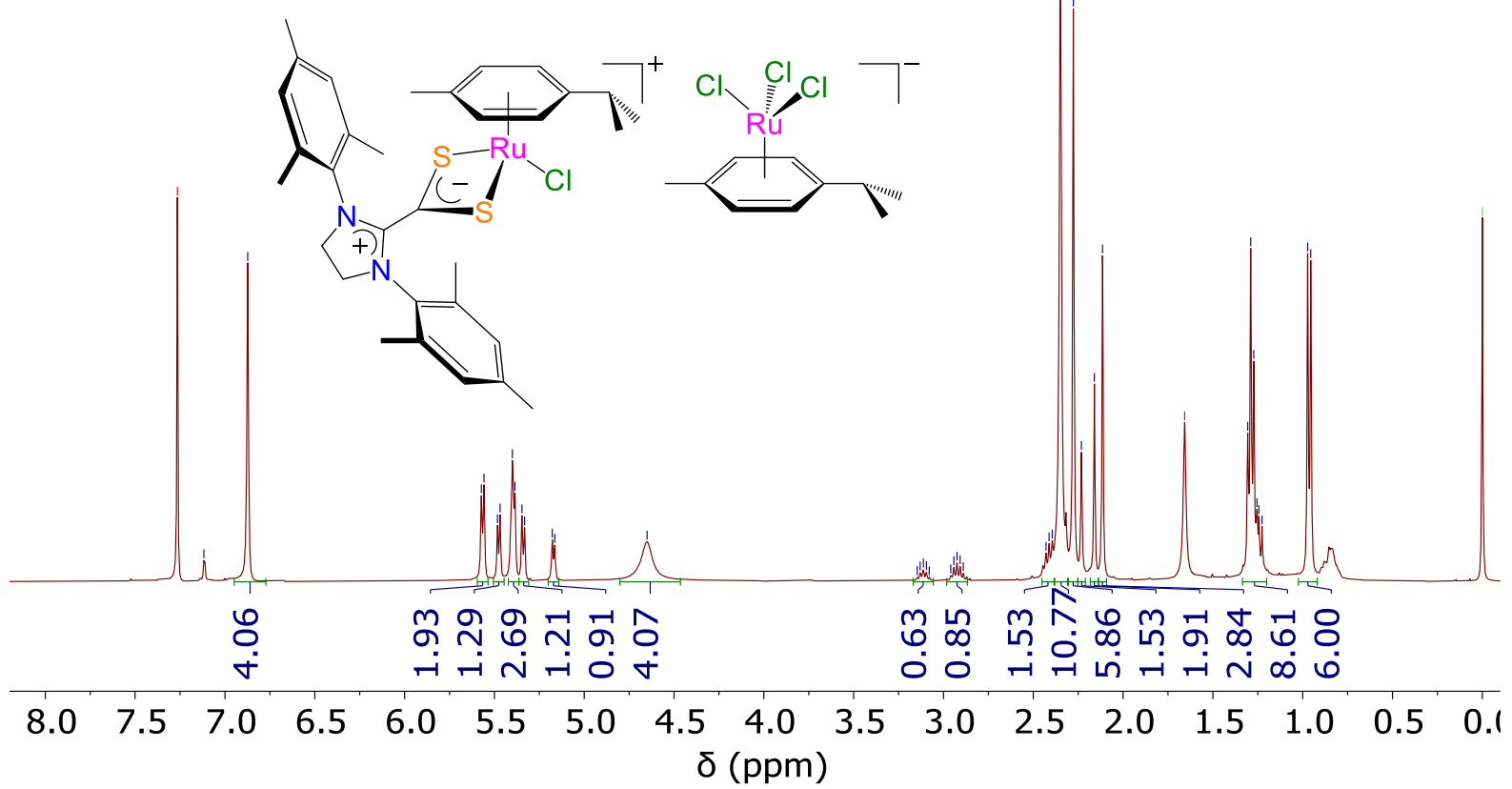

Fig. S33. ${ }^{1} \mathrm{H}$ NMR spectrum $\left(400 \mathrm{MHz}, \mathrm{CDCl}_{3}, 298 \mathrm{~K}\right)$ of $\left[\mathrm{RuCl}(p\right.$-cymene $\left.)\left(\mathrm{S}_{2} \mathrm{C} \cdot \mathrm{SIMes}\right)\right]-$ $\left[\mathrm{RuCl}_{3}(p\right.$-cymene $\left.)\right](\mathbf{3 d})$

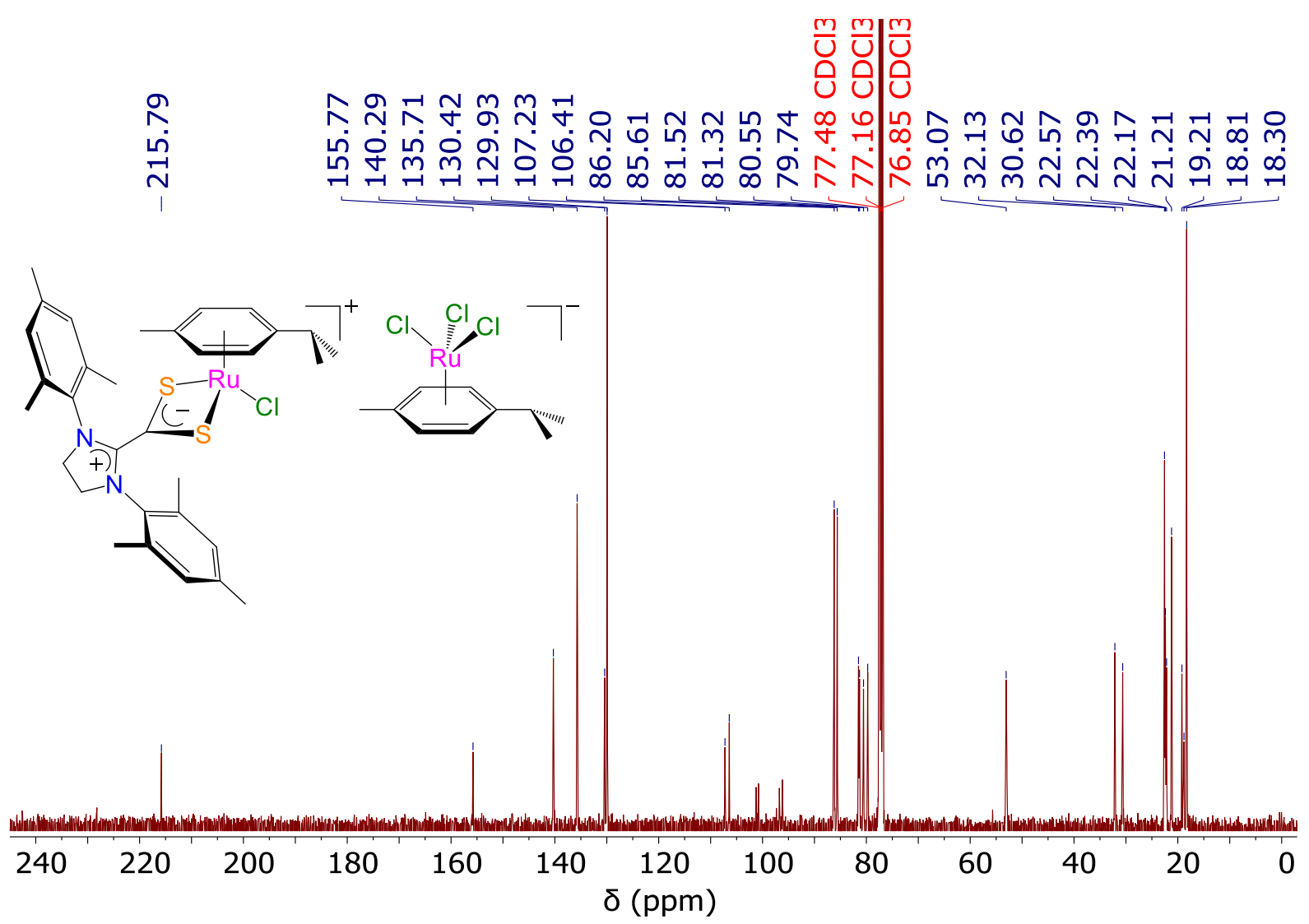


Fig. S34. ${ }^{13} \mathrm{C}\left\{{ }^{1} \mathrm{H}\right\}$ NMR spectrum $\left(101 \mathrm{MHz}, \mathrm{CDCl}_{3}, 298 \mathrm{~K}\right)$ of $[\mathrm{RuCl}(p$-cymene $)$ $\left.\left(\mathrm{S}_{2} \mathrm{C} \cdot \mathrm{SIMes}\right)\right]\left[\mathrm{RuCl}_{3}(p\right.$-cymene $\left.)\right](\mathbf{3 d})$

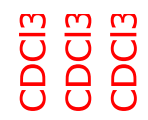

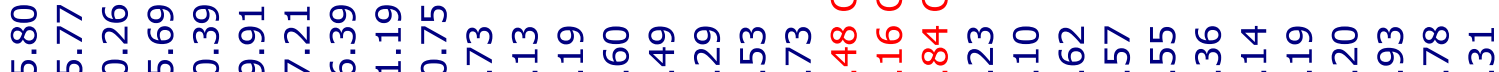

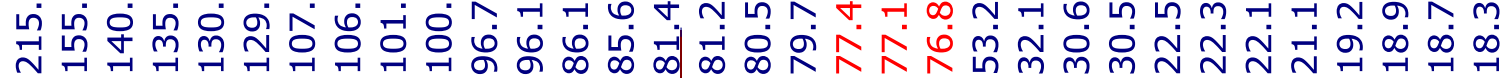

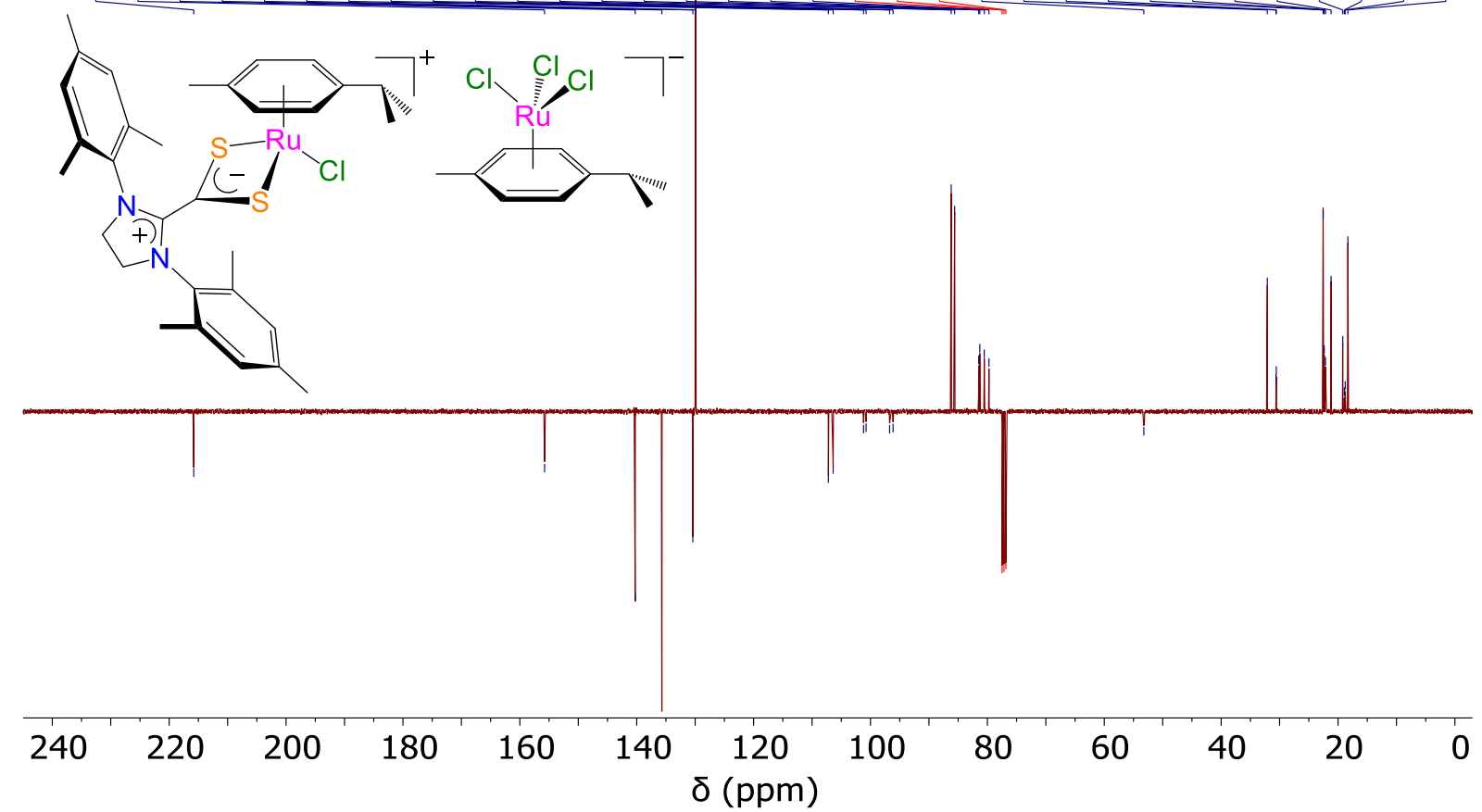

Fig. S35. ${ }^{13} \mathrm{C}\left\{{ }^{1} \mathrm{H}\right\}$ APT NMR spectrum $\left(101 \mathrm{MHz}, \mathrm{CDCl}_{3}, 298 \mathrm{~K}\right)$ of $[\mathrm{RuCl}(p$-cymene $)$ $\left.\left(\mathrm{S}_{2} \mathrm{C} \cdot \mathrm{SIMes}\right)\right]\left[\mathrm{RuCl}_{3}(p\right.$-cymene $\left.)\right](\mathbf{3 d})$

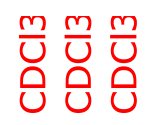

চ ㅎํㅇำ

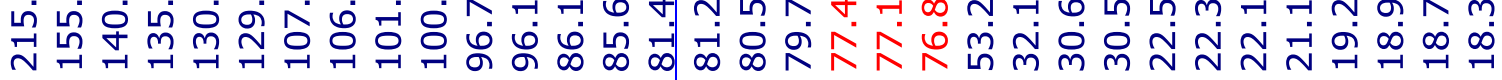

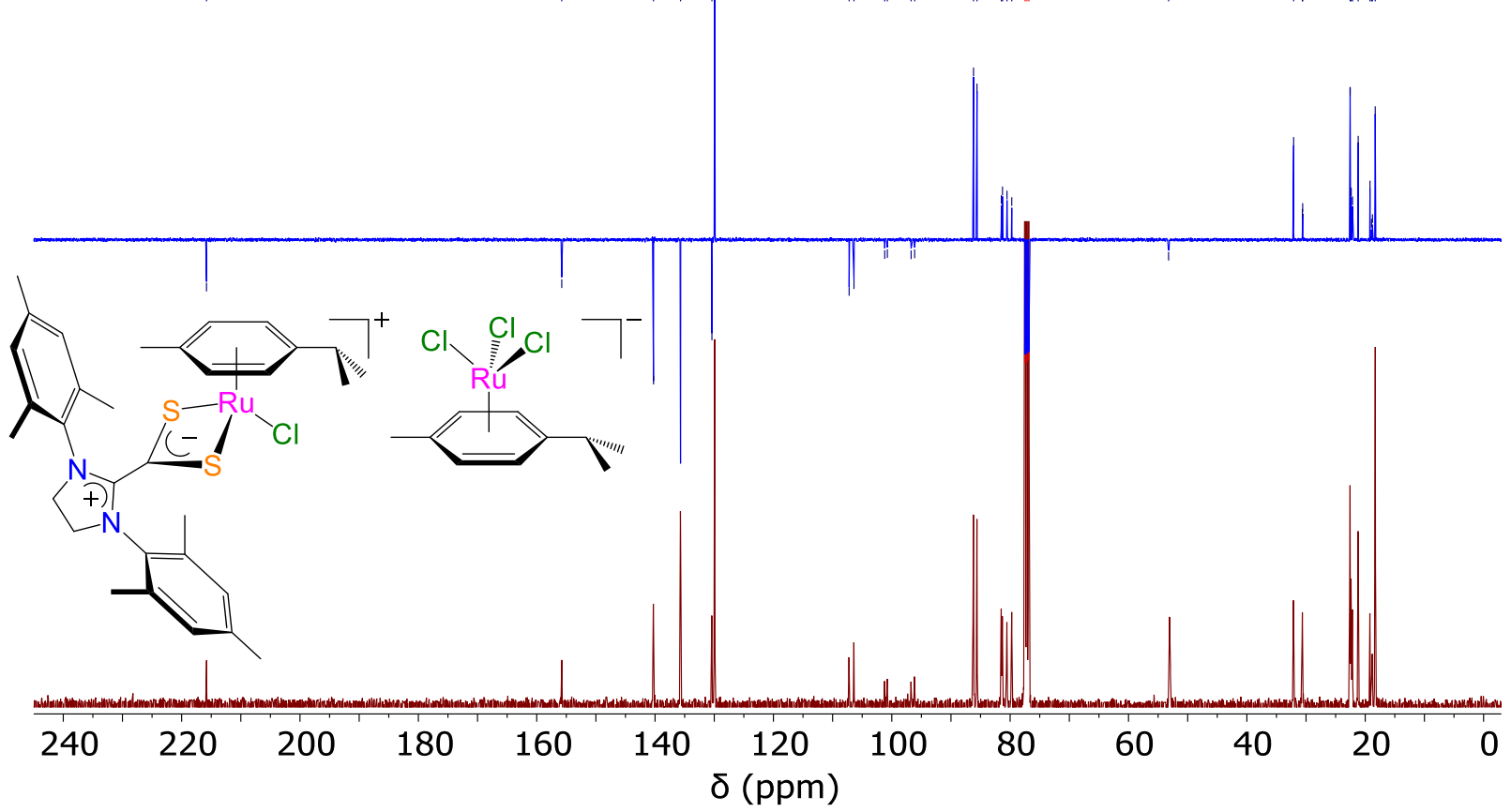


Fig. S36. ${ }^{13} \mathrm{C} \mathrm{CPD}$ and APT NMR spectra (101 $\left.\mathrm{MHz}, \mathrm{CDCl}_{3}, 298 \mathrm{~K}\right)$ of $[\mathrm{RuCl}(p$-cymene)$\left.\left(\mathrm{S}_{2} \mathrm{C} \cdot \mathrm{SIMes}\right)\right]\left[\mathrm{RuCl}_{3}(p\right.$-cymene $\left.)\right](\mathbf{3 d})$<smiles>[O]</smiles>

ซ $\forall$ ๆ

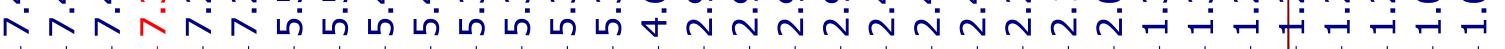

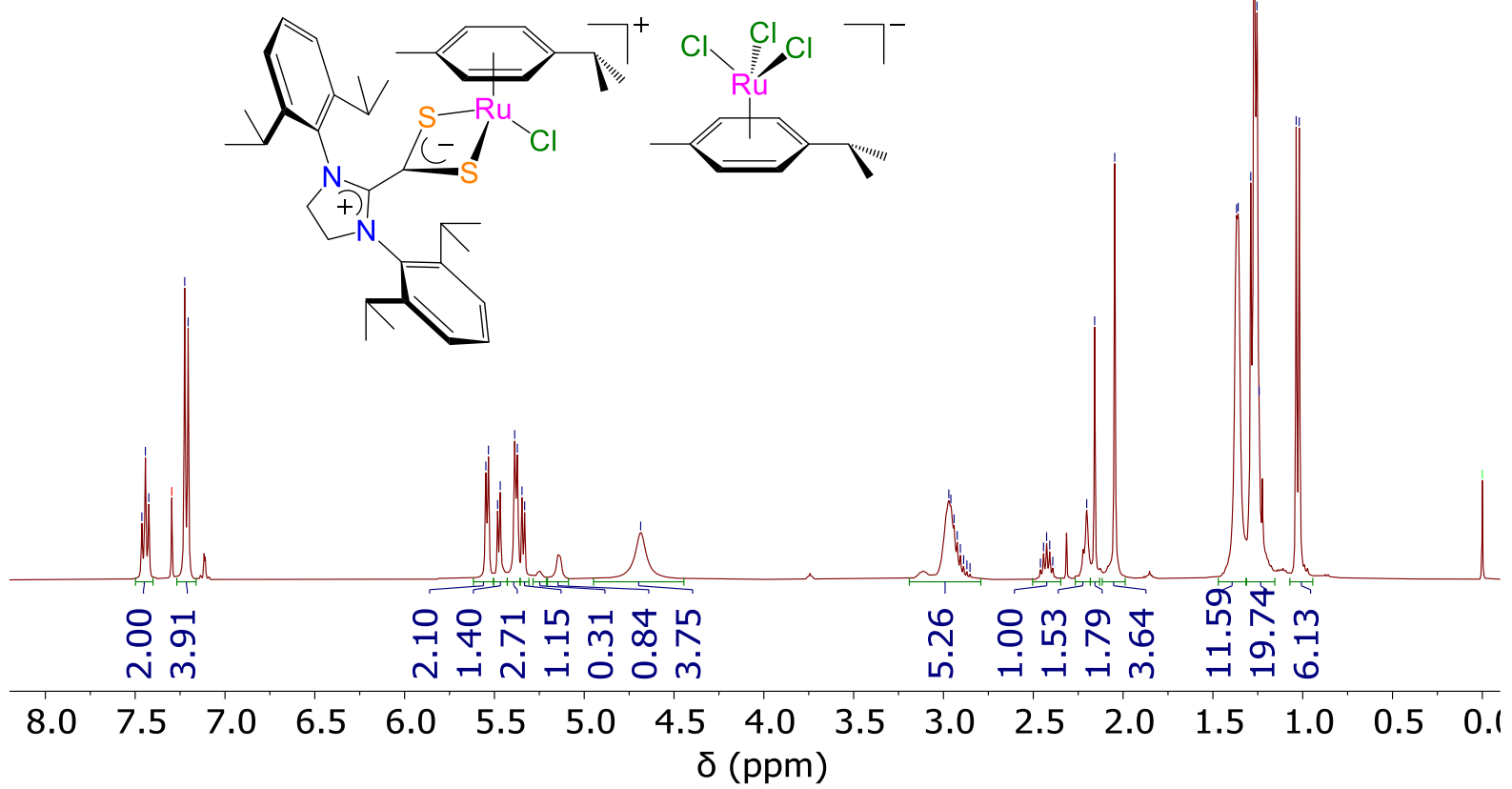

Fig. S37. ${ }^{1} \mathrm{H}$ NMR spectrum (400 $\left.\mathrm{MHz}, \mathrm{CDCl}_{3}, 298 \mathrm{~K}\right)$ of $[\mathrm{RuCl}(p$-cymene)$\left.\left(\mathrm{S}_{2} \mathrm{C} \cdot \mathrm{SIDip}\right)\right]\left[\mathrm{RuCl}_{3}(p\right.$-cymene $\left.)\right](\mathbf{3 e})$

\section{ํㅡㄴ ํㅡㄴ}

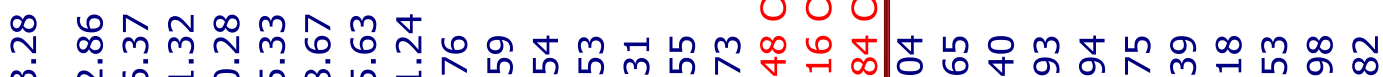
m N
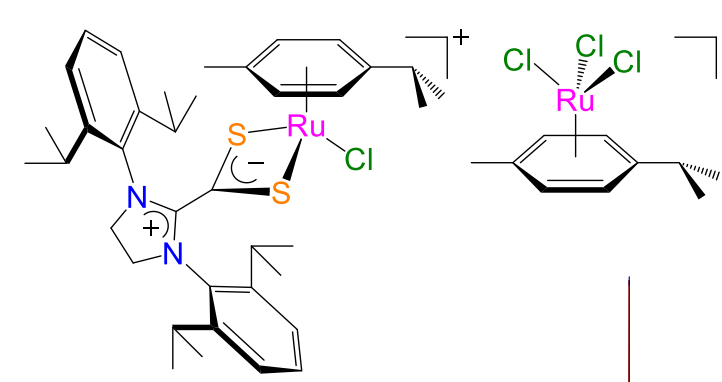
Fig. S38. ${ }^{13} \mathrm{C}\left\{{ }^{1} \mathrm{H}\right\}$ NMR spectrum $\left(101 \mathrm{MHz}, \mathrm{CDCl}_{3}, 298 \mathrm{~K}\right)$ of $[\mathrm{RuCl}(p$-cymene)$\left.\left(\mathrm{S}_{2} \mathrm{C} \cdot \mathrm{SIDip}\right)\right]\left[\mathrm{RuCl}_{3}(p\right.$-cymene $\left.)\right](\mathbf{3 e})$

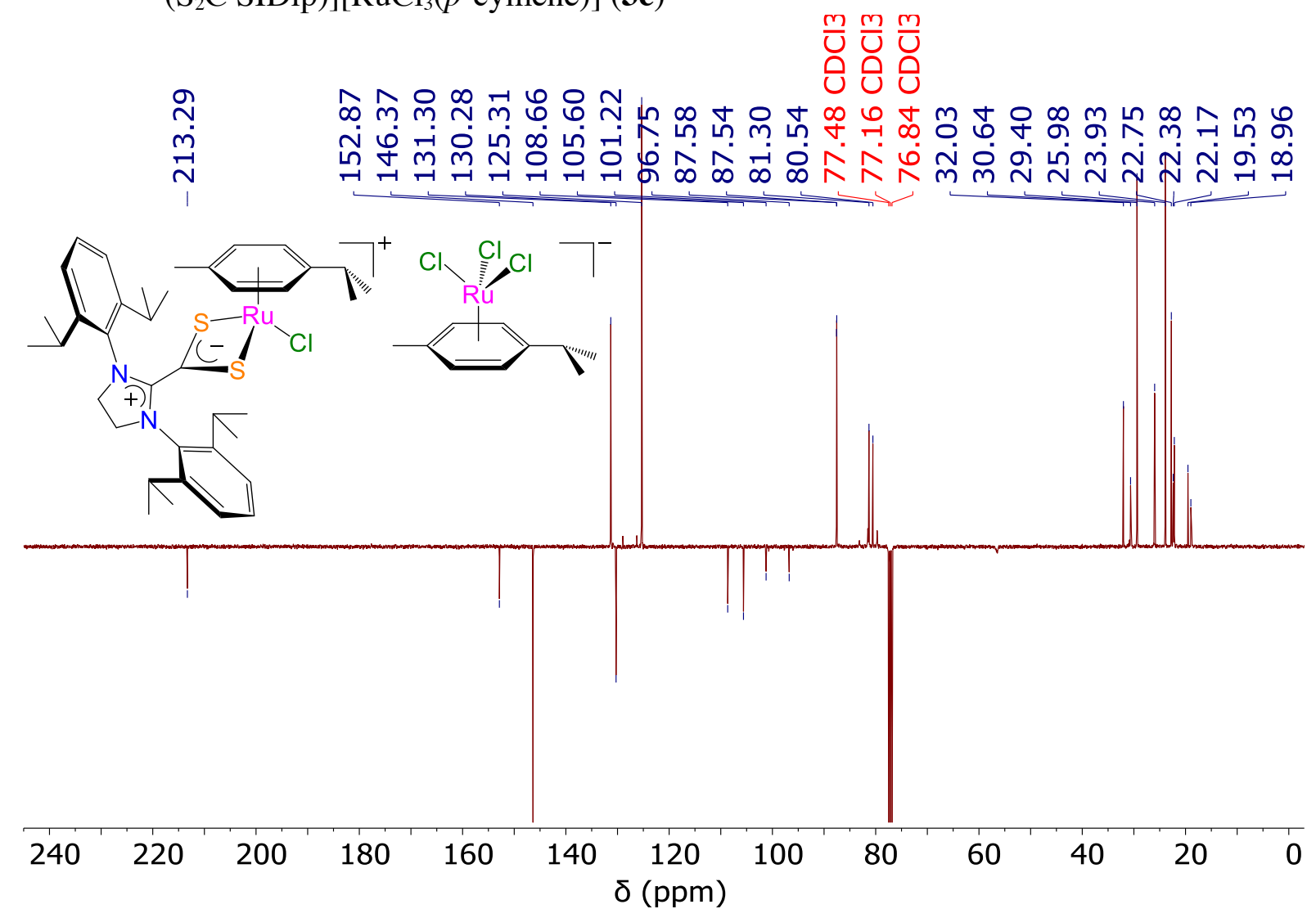

Fig. S39. ${ }^{13} \mathrm{C}\left\{{ }^{1} \mathrm{H}\right\}$ APT NMR spectrum $\left(101 \mathrm{MHz}, \mathrm{CDCl}_{3}, 298 \mathrm{~K}\right)$ of $[\mathrm{RuCl}(p$-cymene $)-$ $\left.\left(\mathrm{S}_{2} \mathrm{C} \cdot \mathrm{SIDip}\right)\right]\left[\mathrm{RuCl}_{3}(p\right.$-cymene $\left.)\right](\mathbf{3 e})$ 


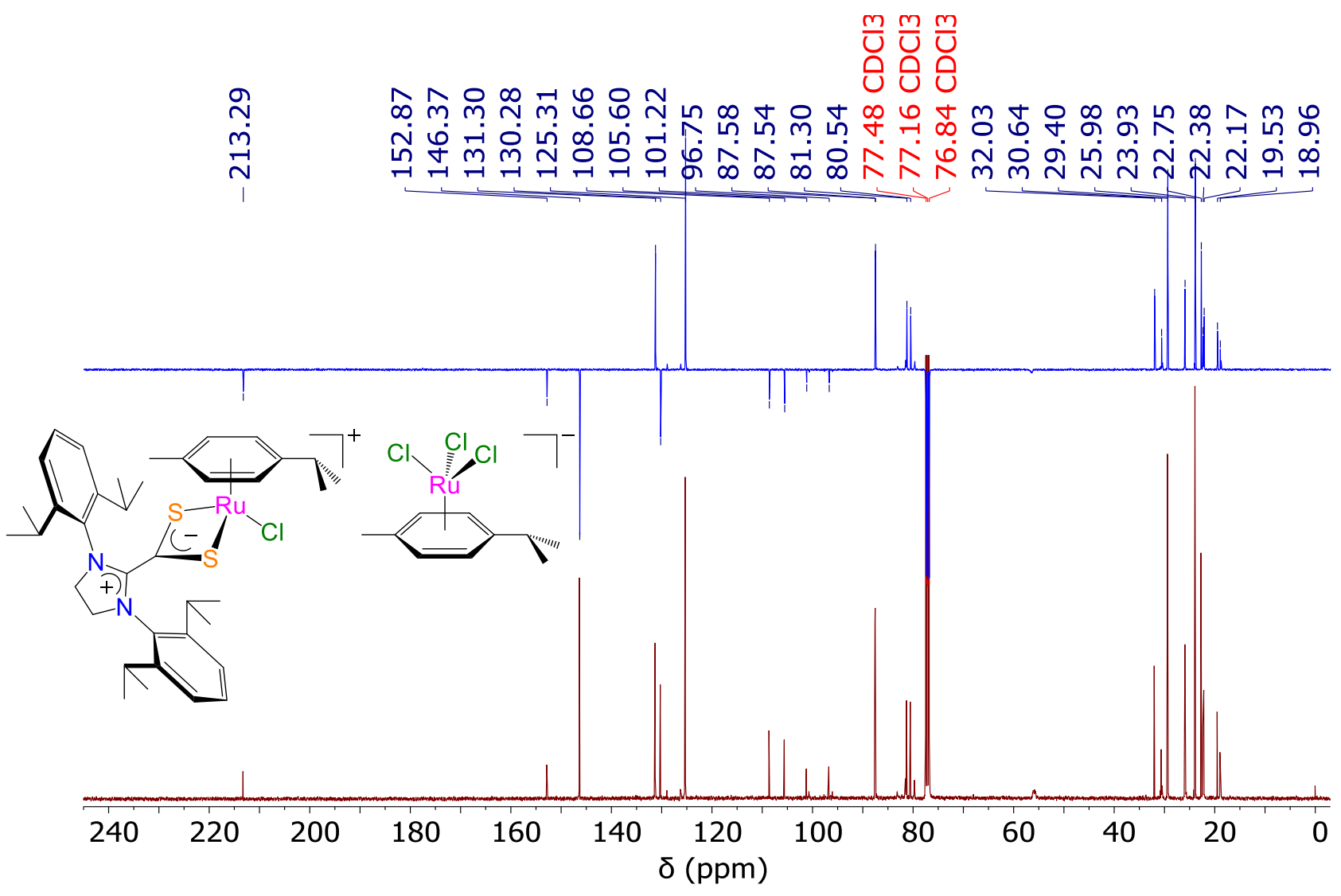

Fig. S40. ${ }^{13} \mathrm{C} \mathrm{CPD}$ and APT NMR spectra (101 MHz, $\left.\mathrm{CDCl}_{3}, 298 \mathrm{~K}\right)$ of $[\mathrm{RuCl}(p$-cymene)$\left.\left(\mathrm{S}_{2} \mathrm{C} \cdot \mathrm{SIDip}\right)\right]\left[\mathrm{RuCl}_{3}(p\right.$-cymene $\left.)\right](\mathbf{3 e})$ 


\section{Part 3 - IR spectra}

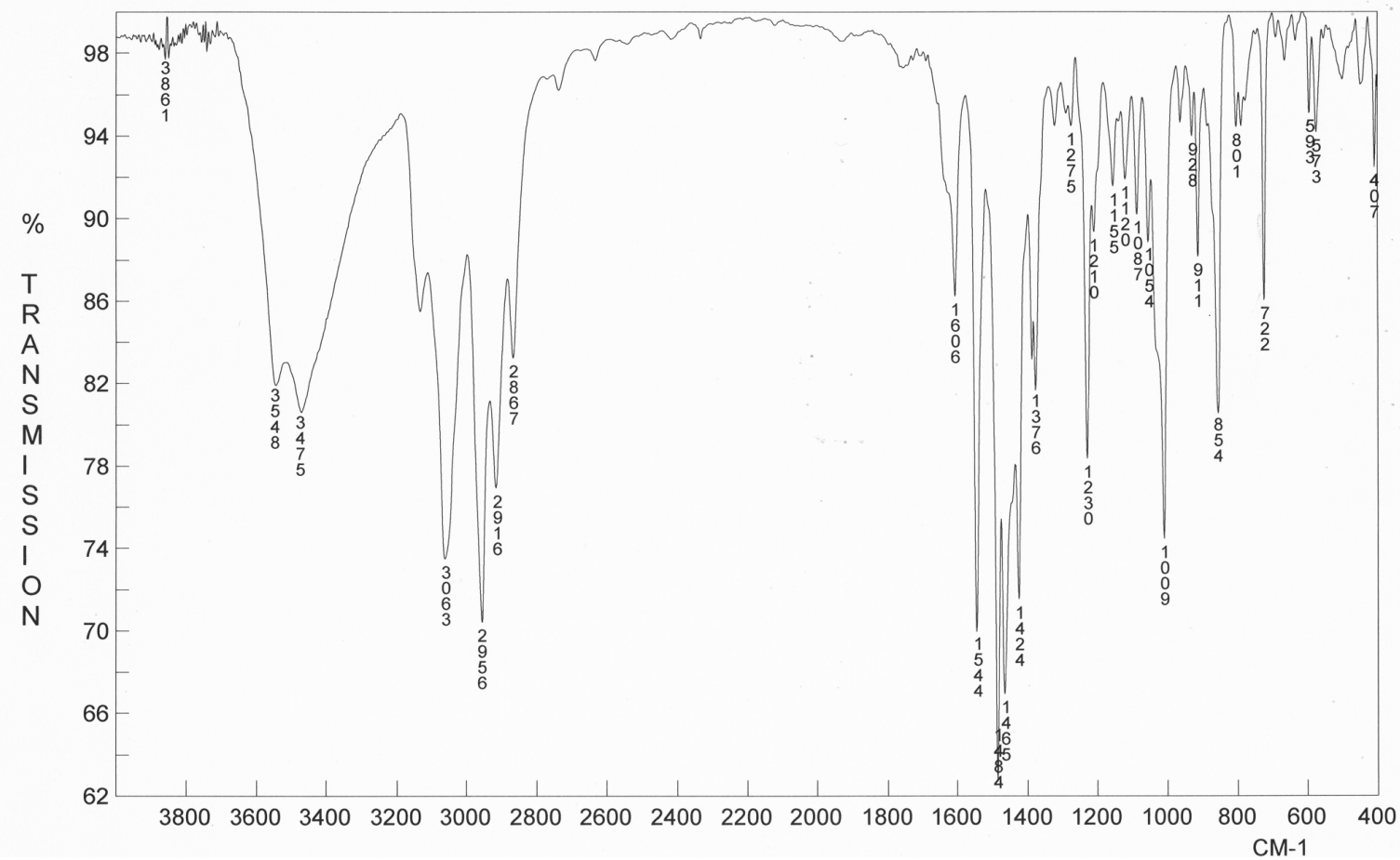

Fig. S41. FT-IR spectrum (KBr) of $\left[\mathrm{RuCl}(p\right.$-cymene $\left.)\left(\mathrm{S}_{2} \mathrm{C} \cdot \mathrm{IMes}\right)\right]\left[\mathrm{RuCl}_{3}(p\right.$-cymene $\left.)\right](3 \mathbf{a})$

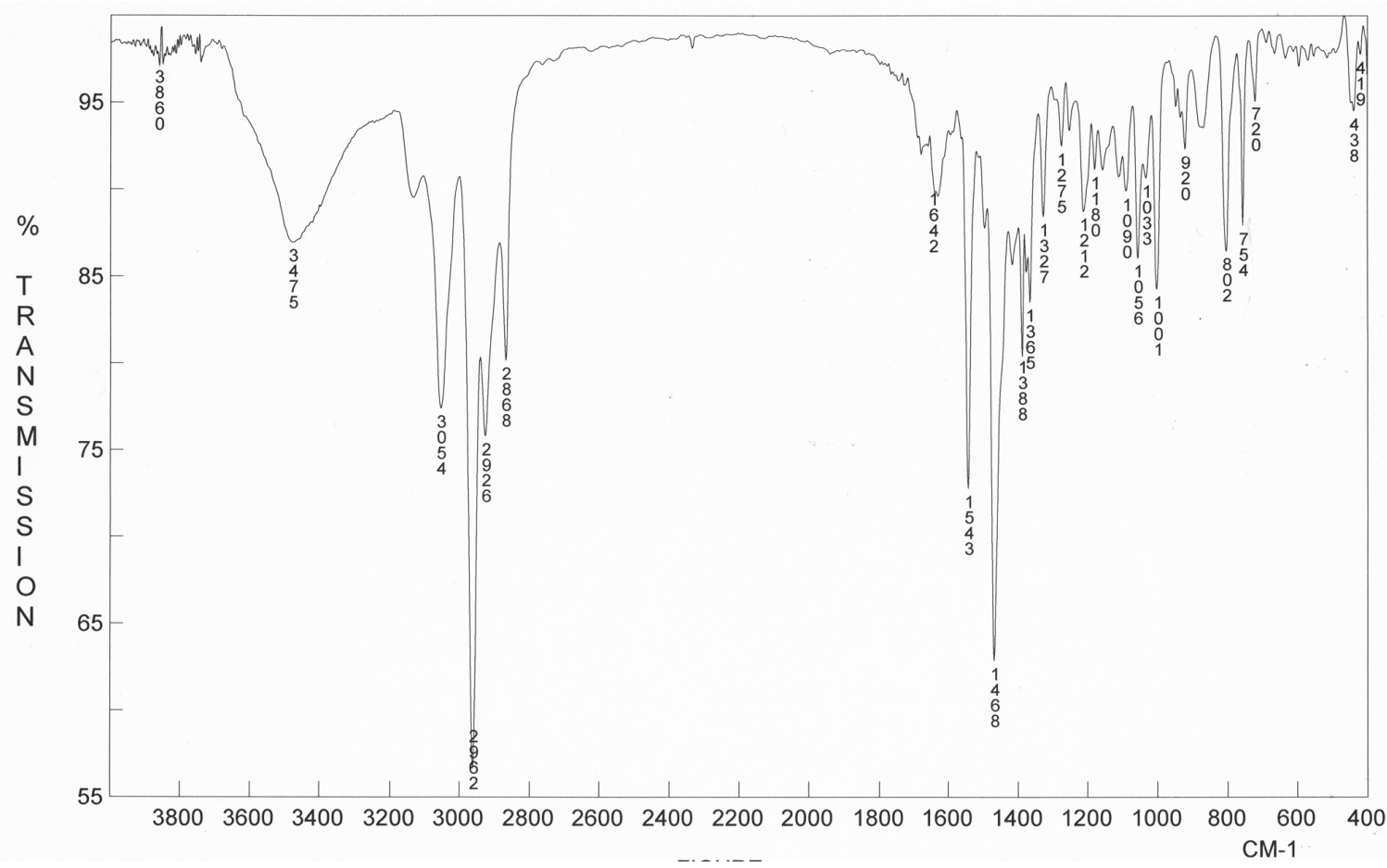

Fig. S42. FT-IR spectrum (KBr) of $\left[\mathrm{RuCl}(p\right.$-cymene $\left.)\left(\mathrm{S}_{2} \mathrm{C} \cdot \mathrm{IDip}\right)\right]\left[\mathrm{RuCl}_{3}(p\right.$-cymene $\left.)\right](\mathbf{3 b})$ 


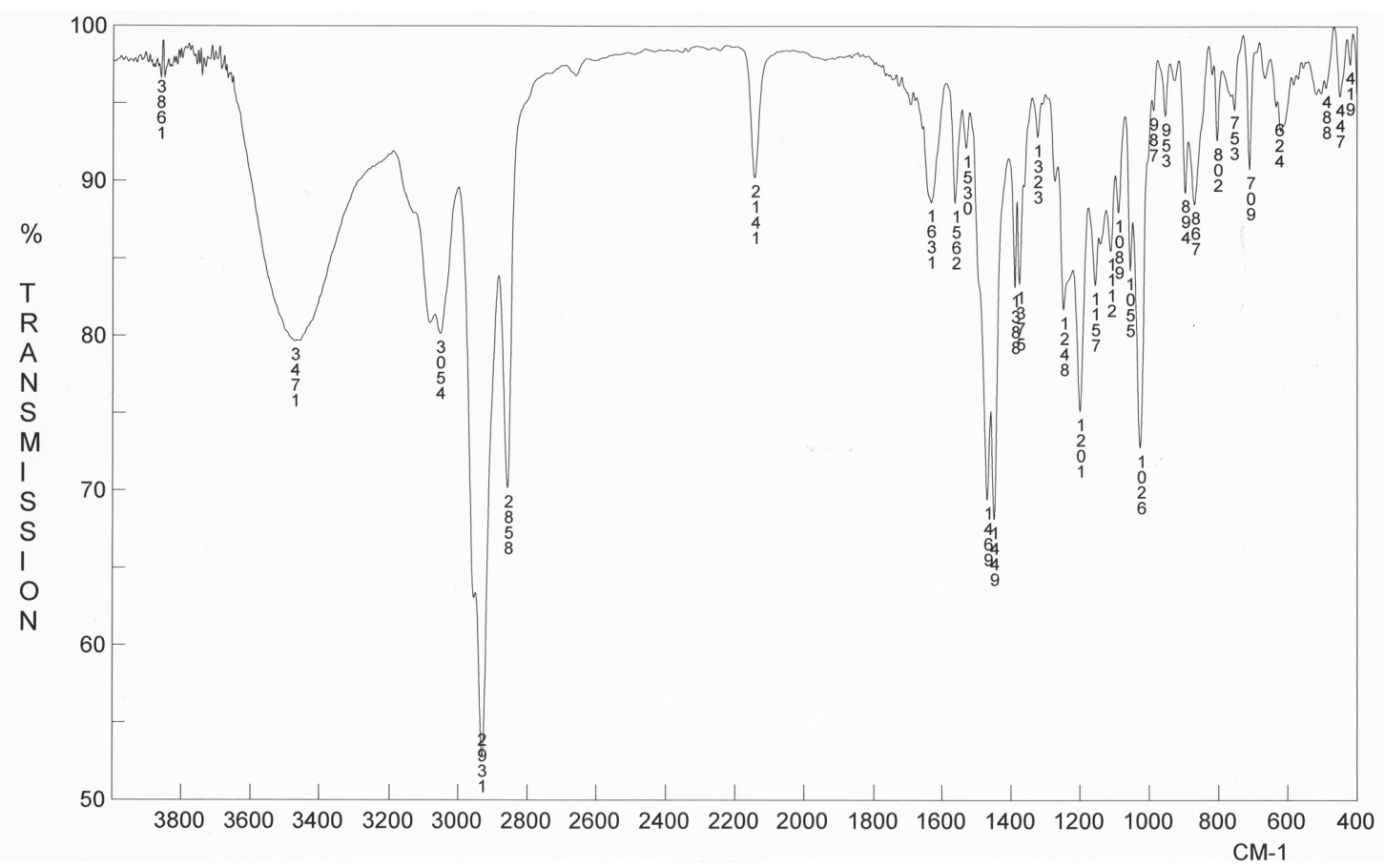

Fig. S43. FT-IR spectrum $(\mathrm{KBr})$ of $\left[\mathrm{RuCl}(p\right.$-cymene $\left.)\left(\mathrm{S}_{2} \mathrm{C} \cdot \mathrm{ICy}\right)\right]\left[\mathrm{RuCl}_{3}(p\right.$-cymene $\left.)\right](3 \mathrm{c})$

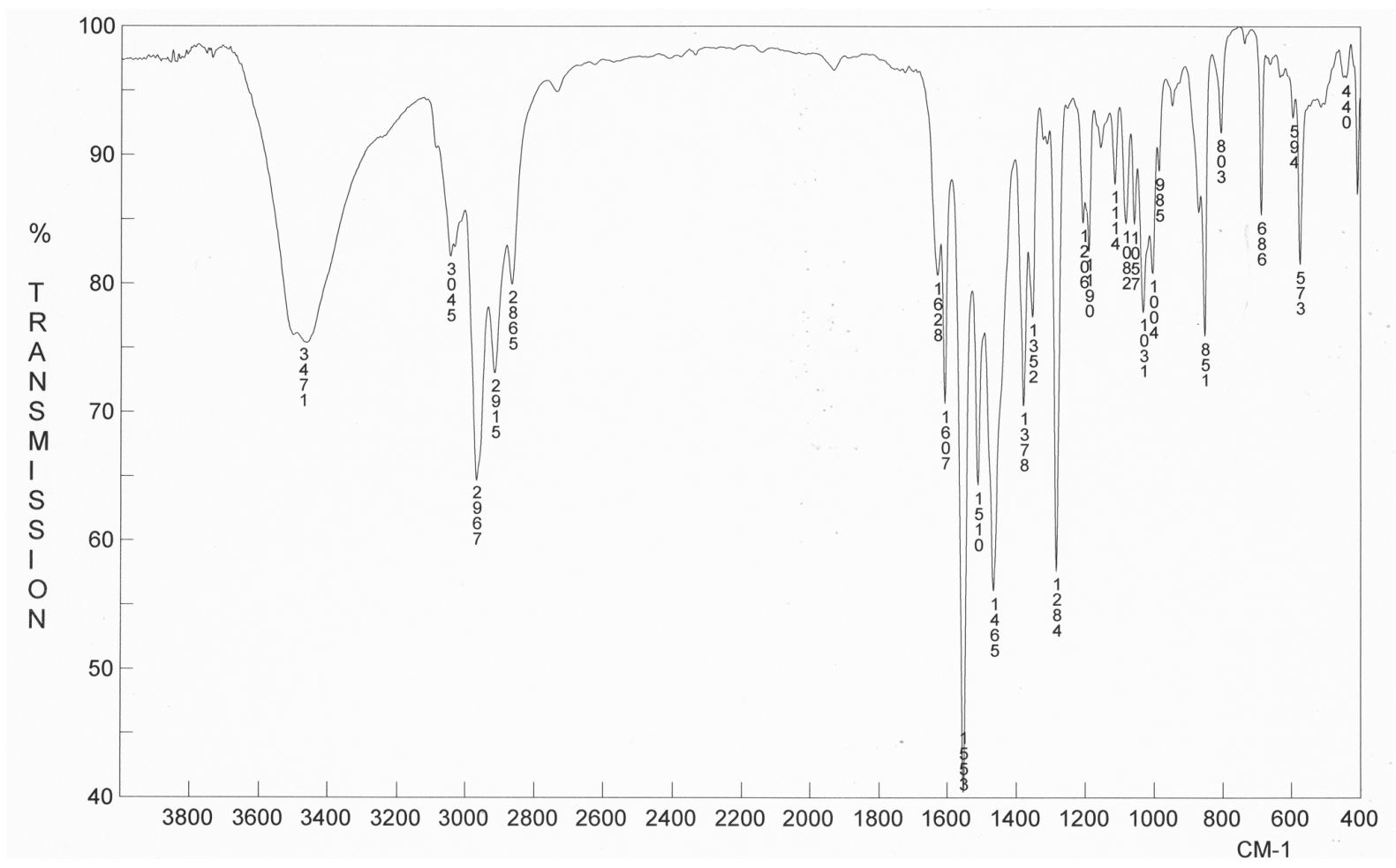

Fig. S44. FT-IR spectrum $(\mathrm{KBr})$ of $\left[\mathrm{RuCl}(p\right.$-cymene $\left.)\left(\mathrm{S}_{2} \mathrm{C} \cdot \mathrm{SIMes}\right)\right]\left[\mathrm{RuCl}_{3}(p\right.$-cymene $\left.)\right](3 \mathrm{~d})$ 


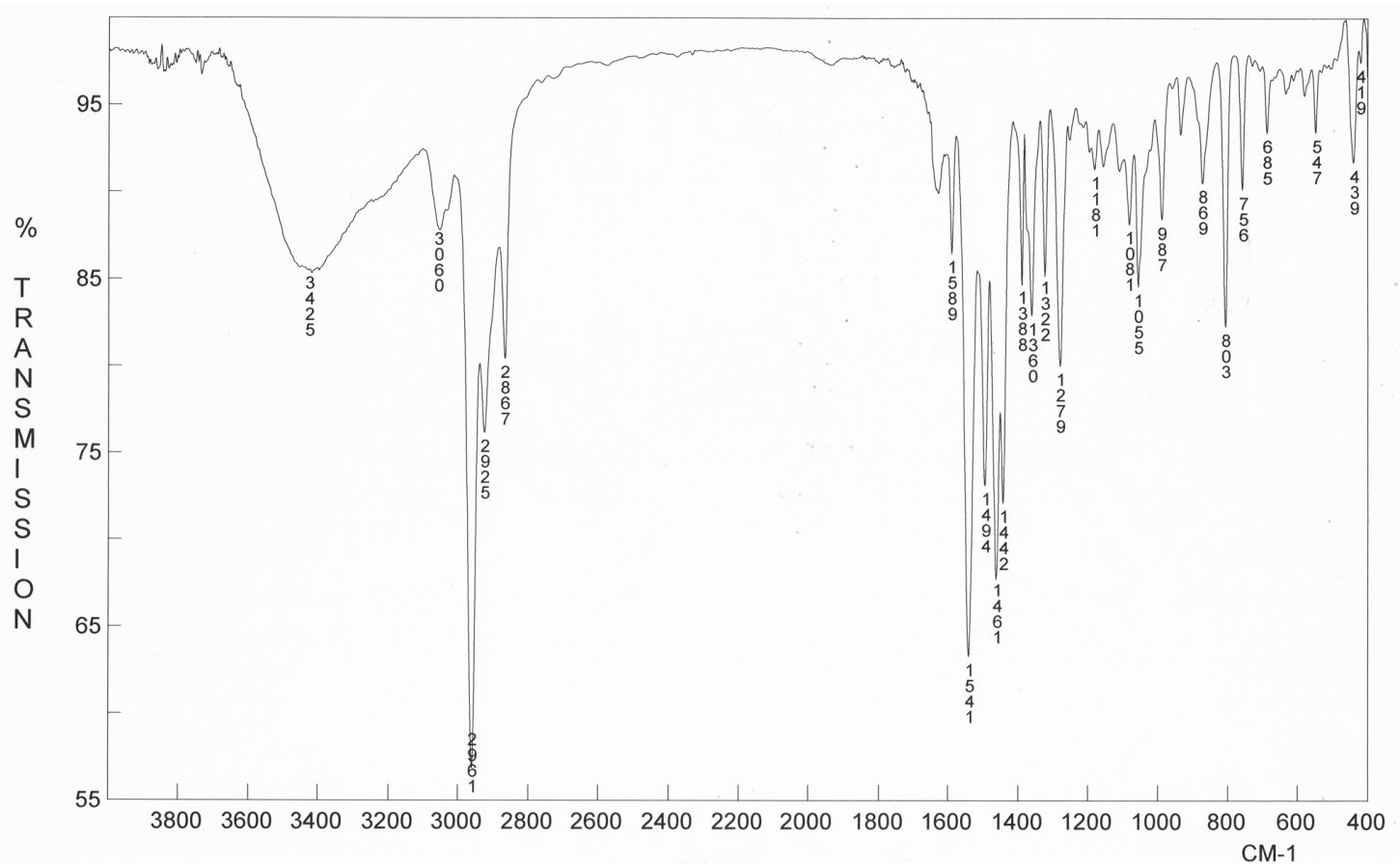

Fig. S45. FT-IR spectrum $(\mathrm{KBr})$ of $\left[\mathrm{RuCl}(p\right.$-cymene $\left.)\left(\mathrm{S}_{2} \mathrm{C} \cdot \mathrm{SIDip}\right)\right]\left[\mathrm{RuCl}_{3}(p\right.$-cymene $\left.)\right](\mathbf{3 e})$ 


\section{Part 4 - Mass spectra}

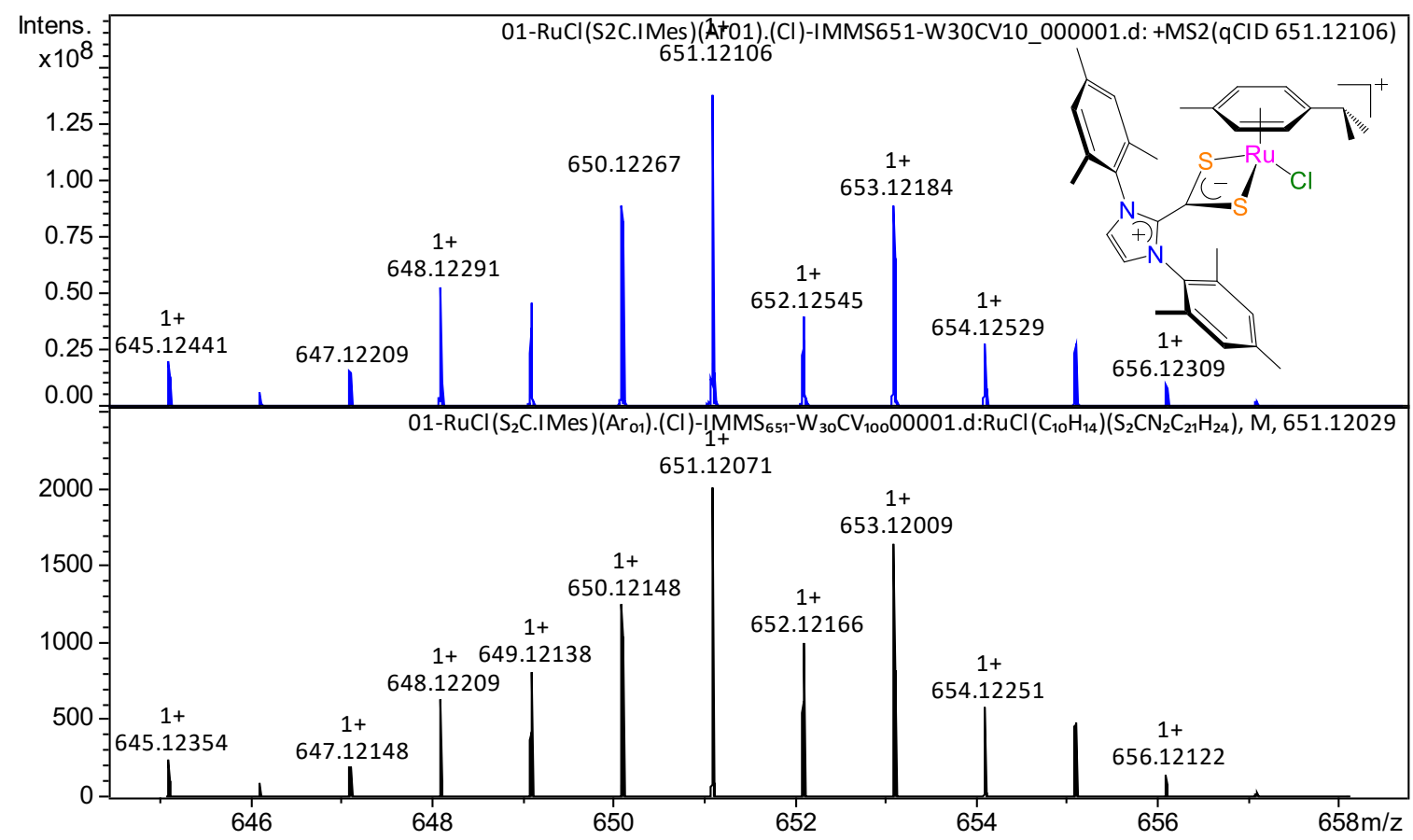

Fig. S46. HR-MS spectrum $\left(\mathrm{CH}_{3} \mathrm{CN}\right.$, positive mode $)$ of $\left[\mathrm{RuCl}(p\right.$-cymene $\left.)\left(\mathrm{S}_{2} \mathrm{C} \cdot \mathrm{IMes}\right)\right] \mathrm{Cl}$ (2a): isotope profile observed by ESI-MS (top) and simulated isotope pattern for the $\left[\mathrm{RuCl}(p \text {-cymene })\left(\mathrm{S}_{2} \mathrm{C} \cdot \mathrm{IMes}\right)\right]^{+}$cation (bottom)

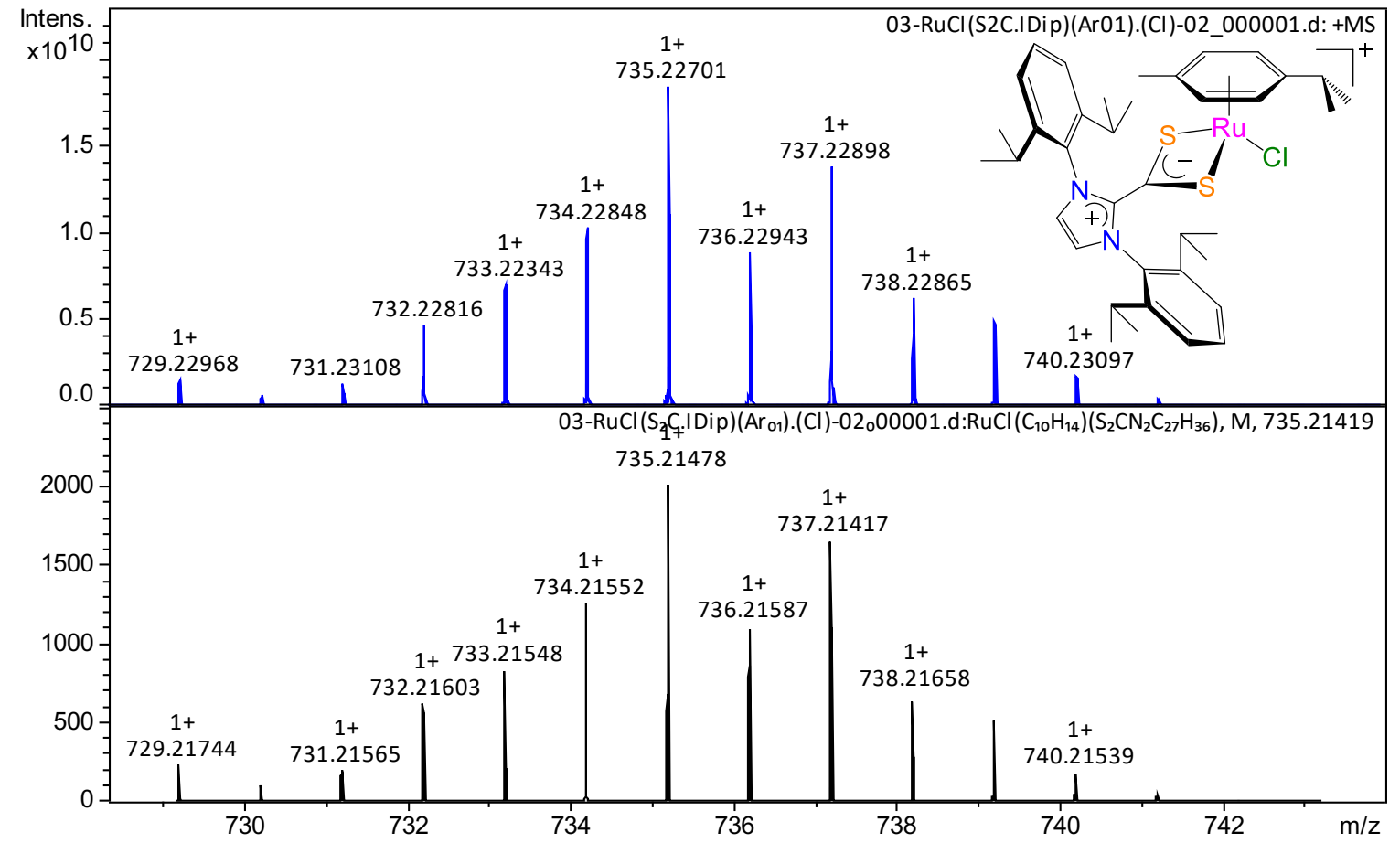

Fig. S47. HR-MS spectrum $\left(\mathrm{CH}_{3} \mathrm{CN}\right.$, positive mode) of $\left[\mathrm{RuCl}(p\right.$-cymene $\left.)\left(\mathrm{S}_{2} \mathrm{C} \cdot \mathrm{IDip}\right)\right] \mathrm{Cl}$ (2b): isotope profile observed by ESI-MS (top) and simulated isotope pattern for the $\left[\mathrm{RuCl}(p \text {-cymene })\left(\mathrm{S}_{2} \mathrm{C} \cdot \mathrm{IDip}\right)\right]^{+}$cation (bottom) 


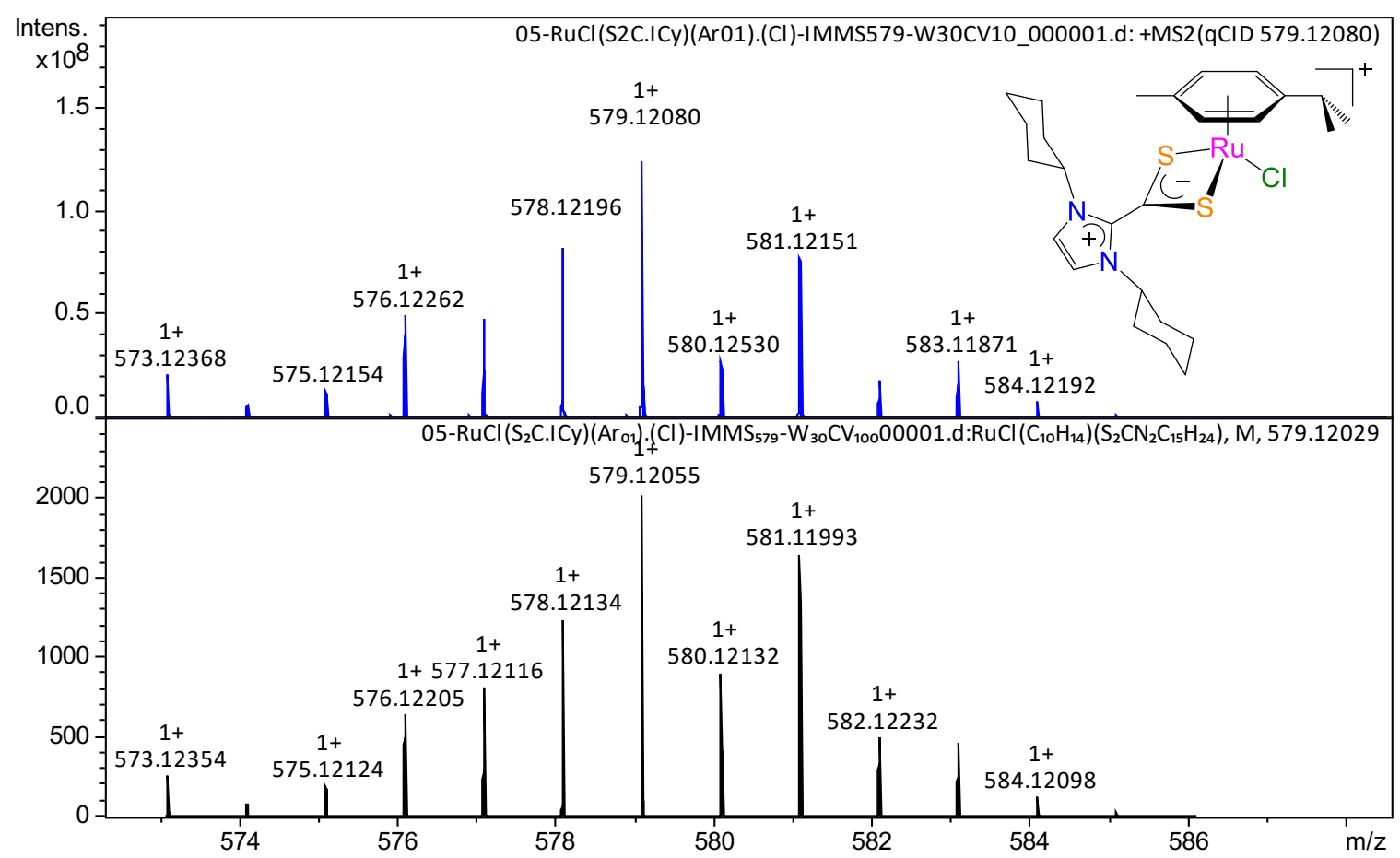

Fig. S48. HR-MS spectrum $\left(\mathrm{CH}_{3} \mathrm{CN}\right.$, positive mode $)$ of $\left[\mathrm{RuCl}(p\right.$-cymene $\left.)\left(\mathrm{S}_{2} \mathrm{C} \cdot \mathrm{ICy}\right)\right] \mathrm{Cl}(\mathbf{2 c})$ : isotope profile observed by ESI-MS (top) and simulated isotope pattern for the $\left[\mathrm{RuCl}(p \text {-cymene })\left(\mathrm{S}_{2} \mathrm{C} \cdot \mathrm{ICy}\right)\right]^{+}$cation (bottom)

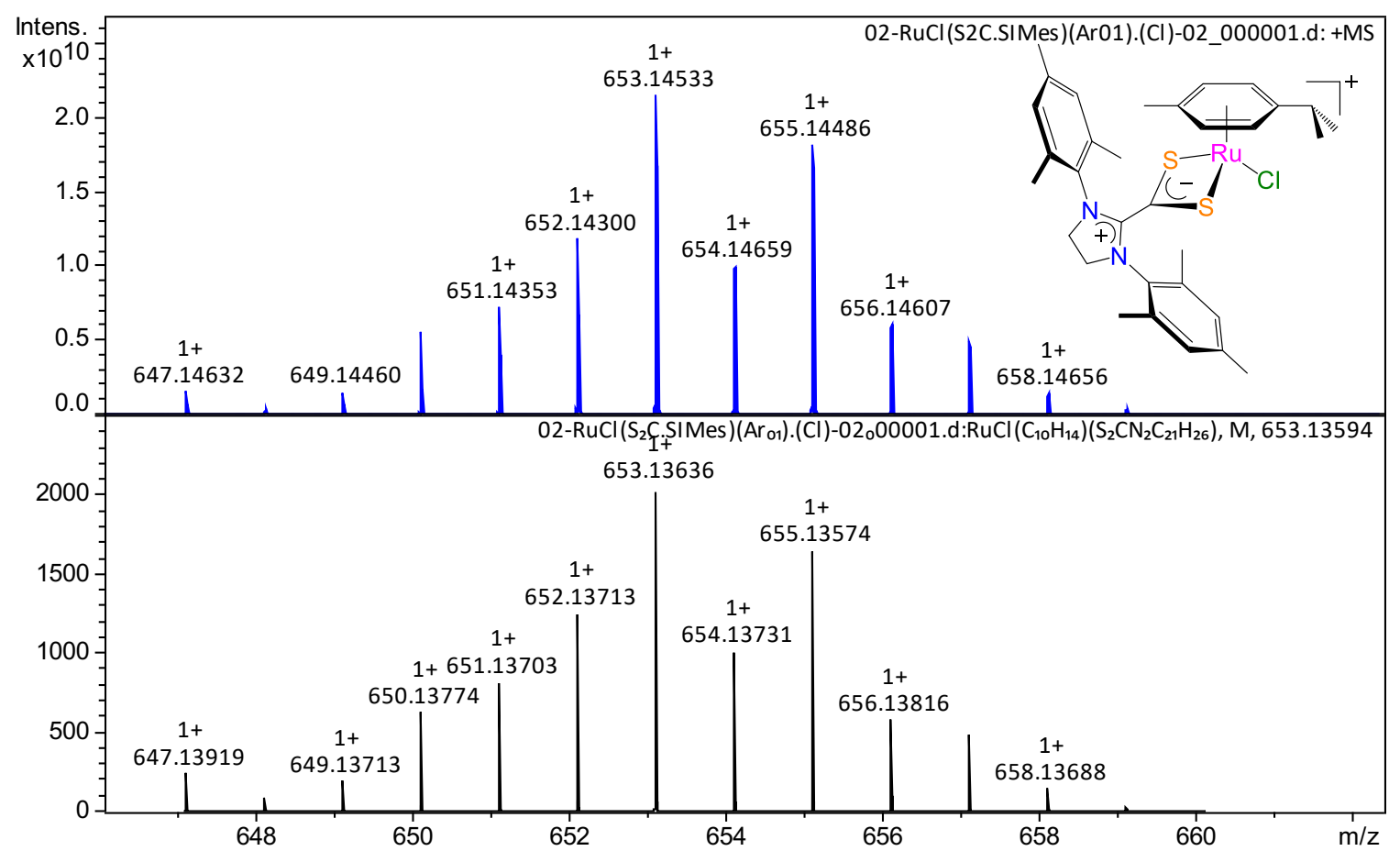

Fig. S49. HR-MS spectrum $\left(\mathrm{CH}_{3} \mathrm{CN}\right.$, positive mode $)$ of $\left[\mathrm{RuCl}(p\right.$-cymene $\left.)\left(\mathrm{S}_{2} \mathrm{C} \cdot \mathrm{SIMes}\right)\right] \mathrm{Cl}$ (2d): isotope profile observed by ESI-MS (top) and simulated isotope pattern for the $\left[\mathrm{RuCl}(p \text {-cymene })\left(\mathrm{S}_{2} \mathrm{C} \cdot \mathrm{SIMes}\right)\right]^{+}$cation (bottom) 


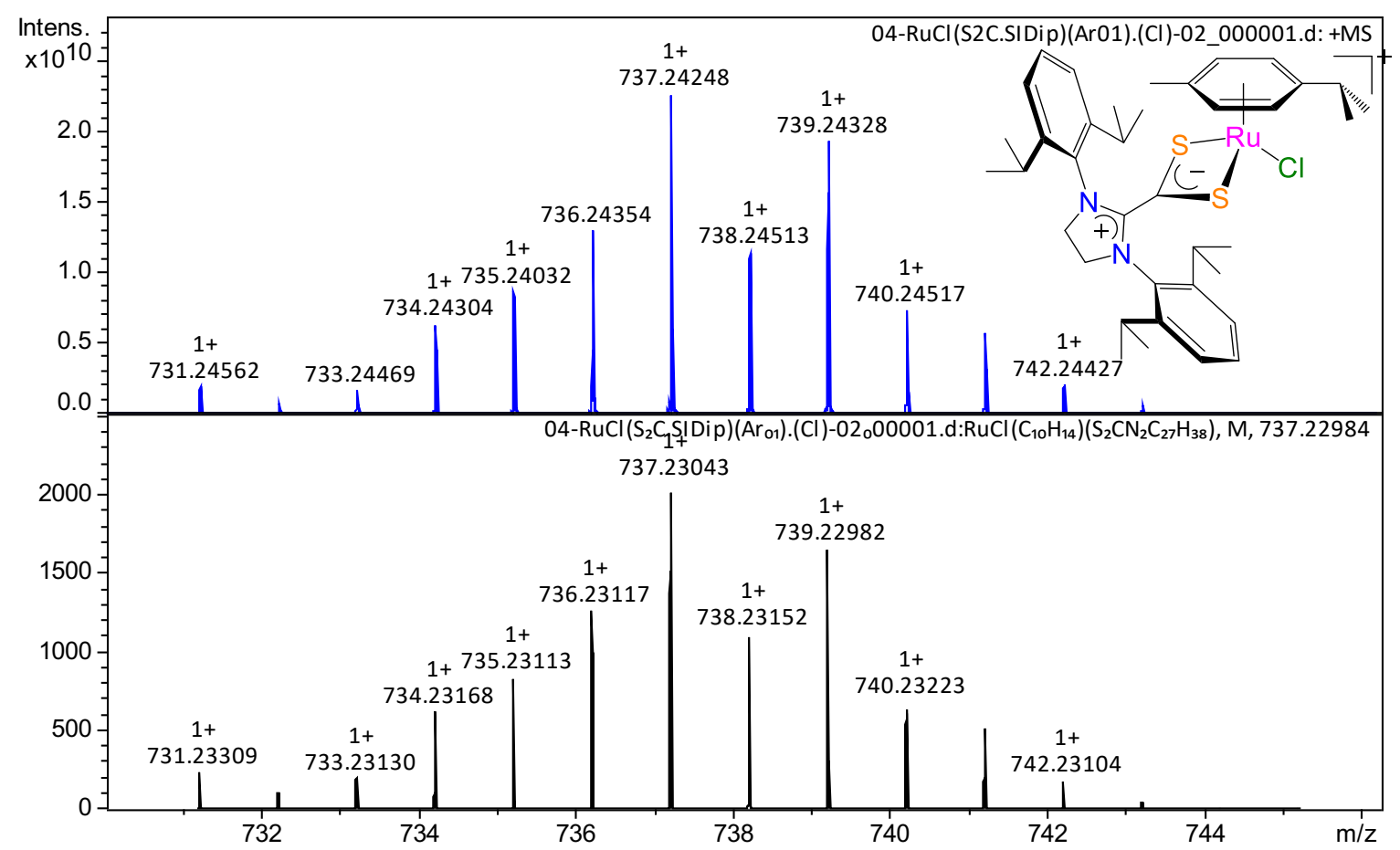

Fig. S50. HR-MS spectrum $\left(\mathrm{CH}_{3} \mathrm{CN}\right.$, positive mode $)$ of $\left[\mathrm{RuCl}(p\right.$-cymene $\left.)\left(\mathrm{S}_{2} \mathrm{C} \cdot \mathrm{SIDip}\right)\right] \mathrm{Cl}$ (2e): isotope profile observed by ESI-MS (top) and simulated isotope pattern for the $\left[\mathrm{RuCl}(p \text {-cymene })\left(\mathrm{S}_{2} \mathrm{C} \cdot \mathrm{SIDip}\right)\right]^{+}$cation (bottom) 


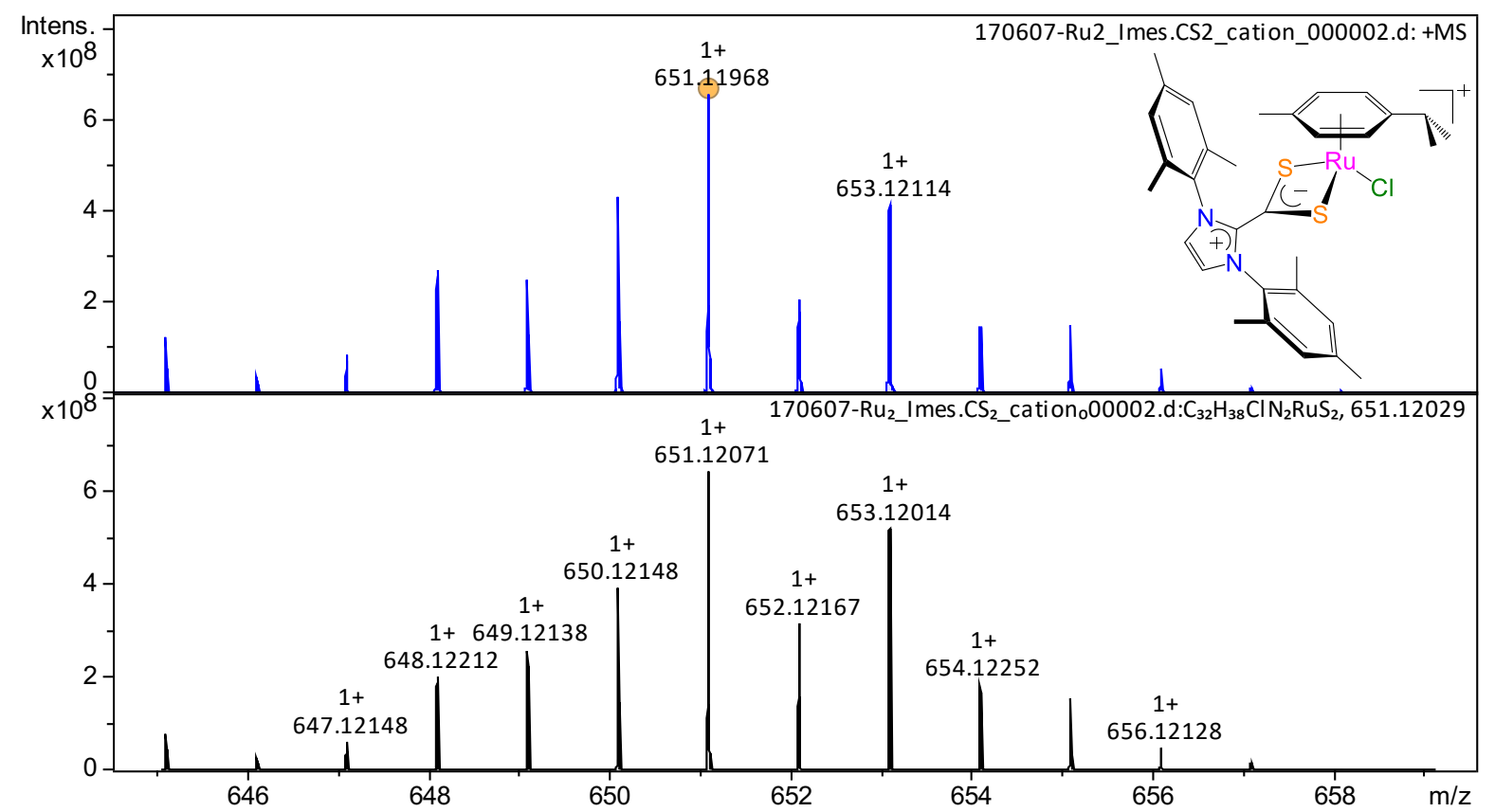

Fig. S51. HR-MS spectrum $\left(\mathrm{CH}_{3} \mathrm{CN}\right.$, positive mode $)$ of $\left[\mathrm{RuCl}(p\right.$-cymene $\left.)\left(\mathrm{S}_{2} \mathrm{C} \cdot \mathrm{IMes}\right)\right]-$ $\left[\mathrm{RuCl}_{3}(p\right.$-cymene)] (3a): isotope profile observed by ESI-MS (top) and simulated isotope pattern for the $\left[\mathrm{RuCl}(p \text {-cymene })\left(\mathrm{S}_{2} \mathrm{C} \cdot \mathrm{IMes}\right)\right]^{+}$cation (bottom)

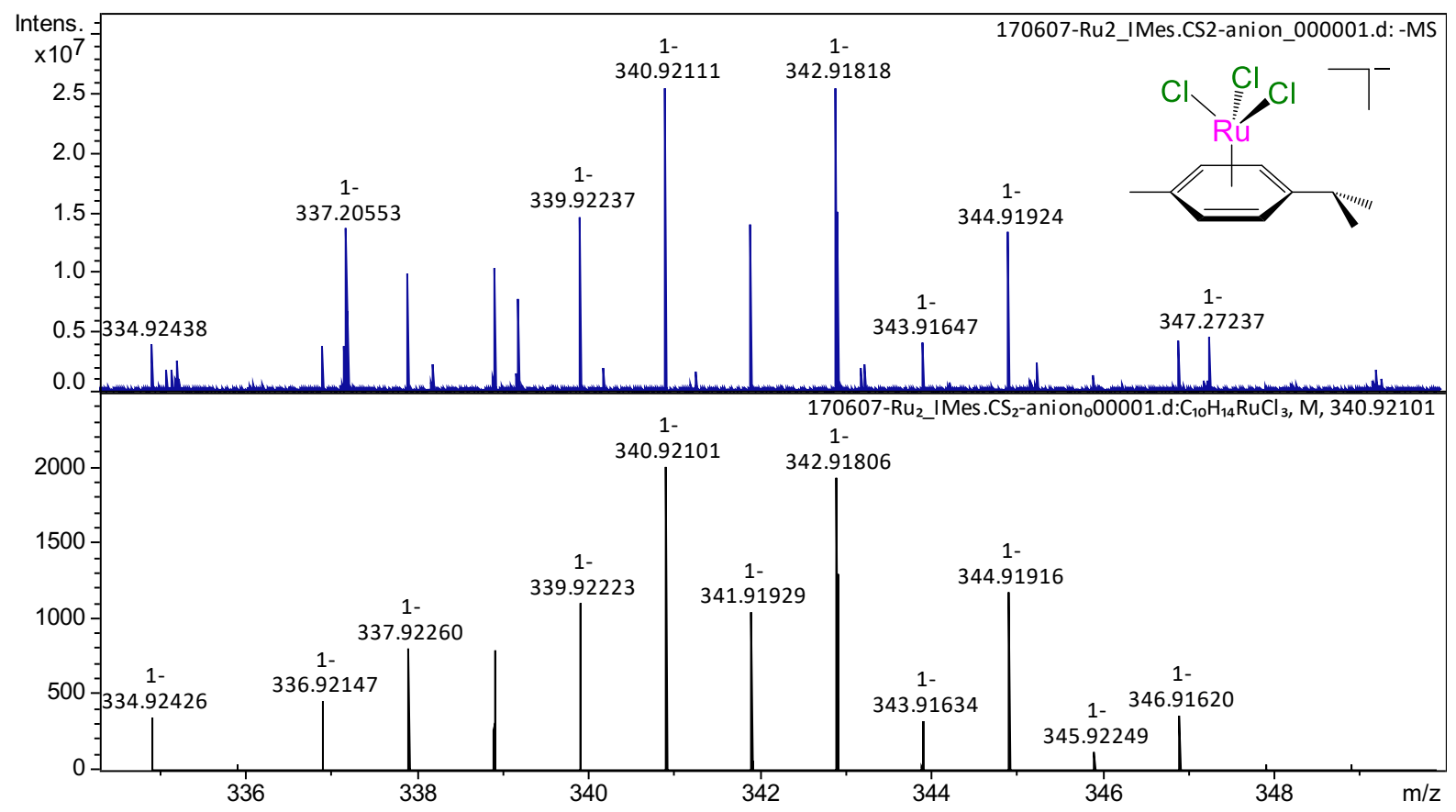

Fig. S52. HR-MS spectrum $\left(\mathrm{CH}_{3} \mathrm{CN}\right.$, negative mode $)$ of $\left[\mathrm{RuCl}(p\right.$-cymene $\left.)\left(\mathrm{S}_{2} \mathrm{C} \cdot \mathrm{IMes}\right)\right]$ $\left[\mathrm{RuCl}_{3}(p\right.$-cymene)] (3a): isotope profile observed by ESI-MS (top) and simulated isotope pattern for the $\left[\mathrm{RuCl}_{3}(p \text {-cymene) }]^{-}\right.$anion (bottom) 


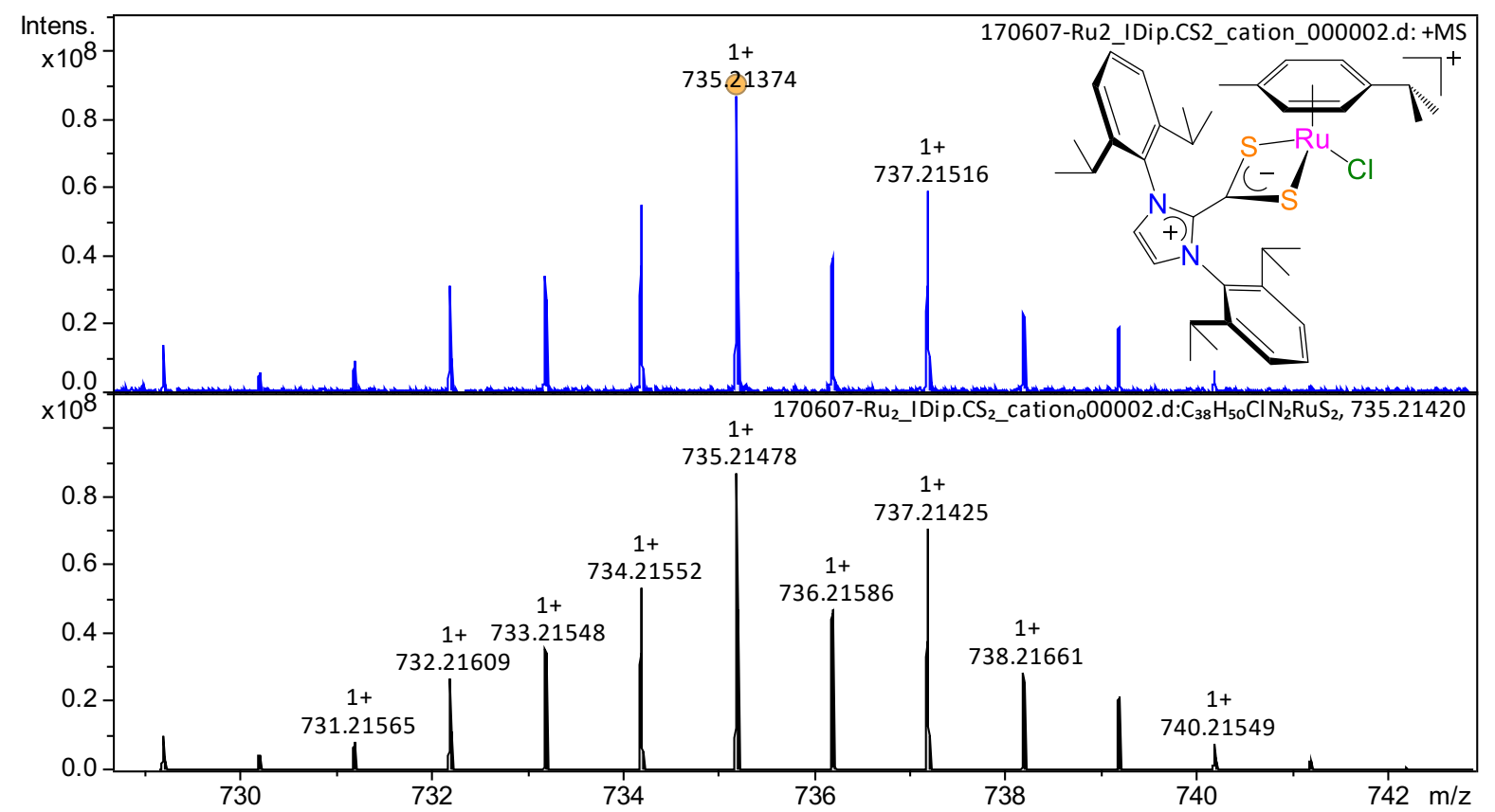

Fig. S53. HR-MS spectrum $\left(\mathrm{CH}_{3} \mathrm{CN}\right.$, positive mode) of $\left[\mathrm{RuCl}(p\right.$-cymene $\left.)\left(\mathrm{S}_{2} \mathrm{C} \cdot \mathrm{IDip}\right)\right]-$ $\left[\mathrm{RuCl}_{3}(p\right.$-cymene)] (3b): isotope profile observed by ESI-MS (top) and simulated isotope pattern for the $\left[\mathrm{RuCl}(p \text {-cymene })\left(\mathrm{S}_{2} \mathrm{C} \cdot \mathrm{IDip}\right)\right]^{+}$cation (bottom)

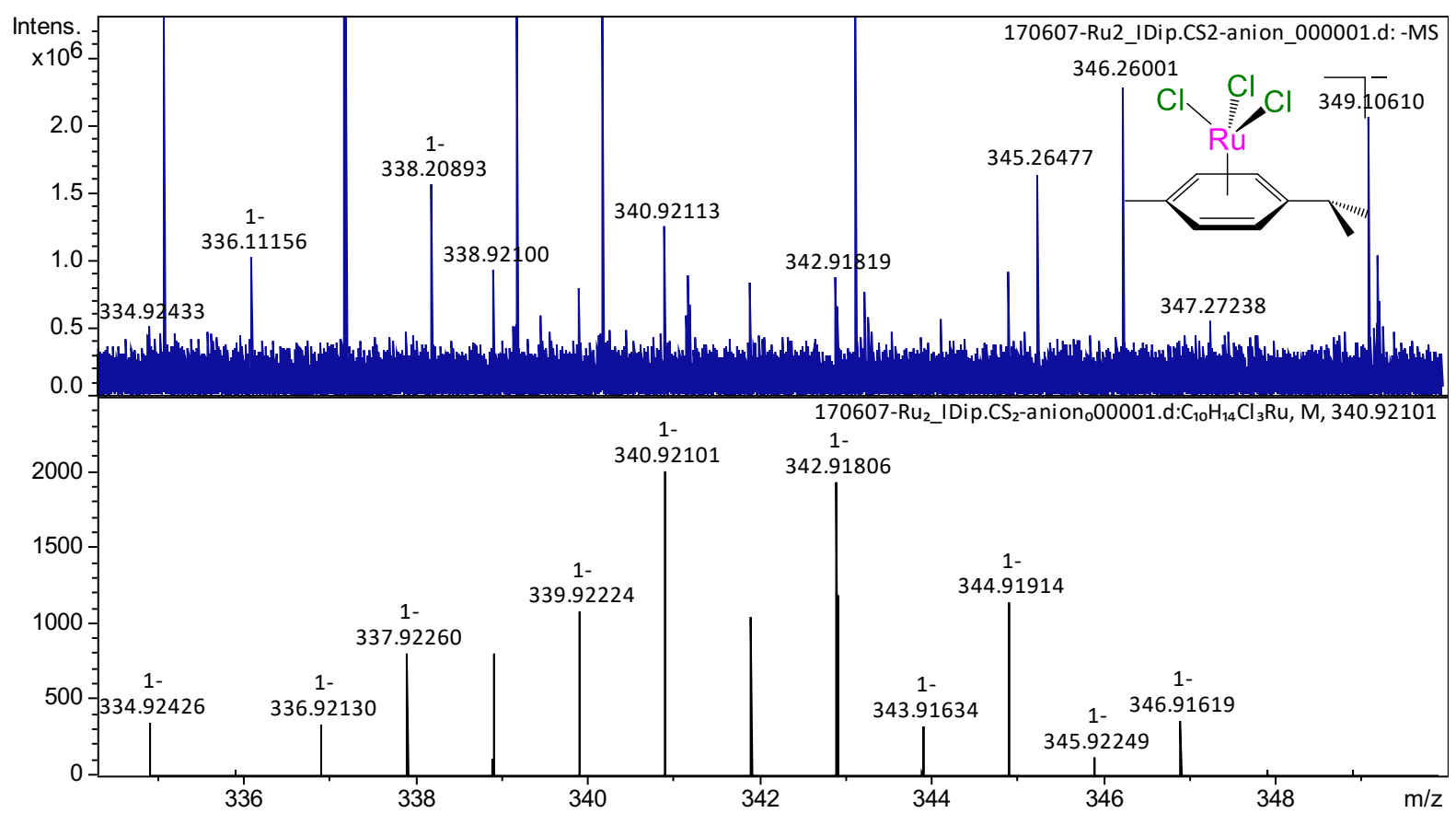

Fig. S54. HR-MS spectrum $\left(\mathrm{CH}_{3} \mathrm{CN}\right.$, negative mode $)$ of $\left[\mathrm{RuCl}(p\right.$-cymene $\left.)\left(\mathrm{S}_{2} \mathrm{C} \cdot \mathrm{IDip}\right)\right]-$ $\left[\mathrm{RuCl}_{3}(p\right.$-cymene)] (3b): isotope profile observed by ESI-MS (top) and simulated isotope pattern for the $\left[\mathrm{RuCl}_{3}(p \text {-cymene })\right]^{-}$anion (bottom) 


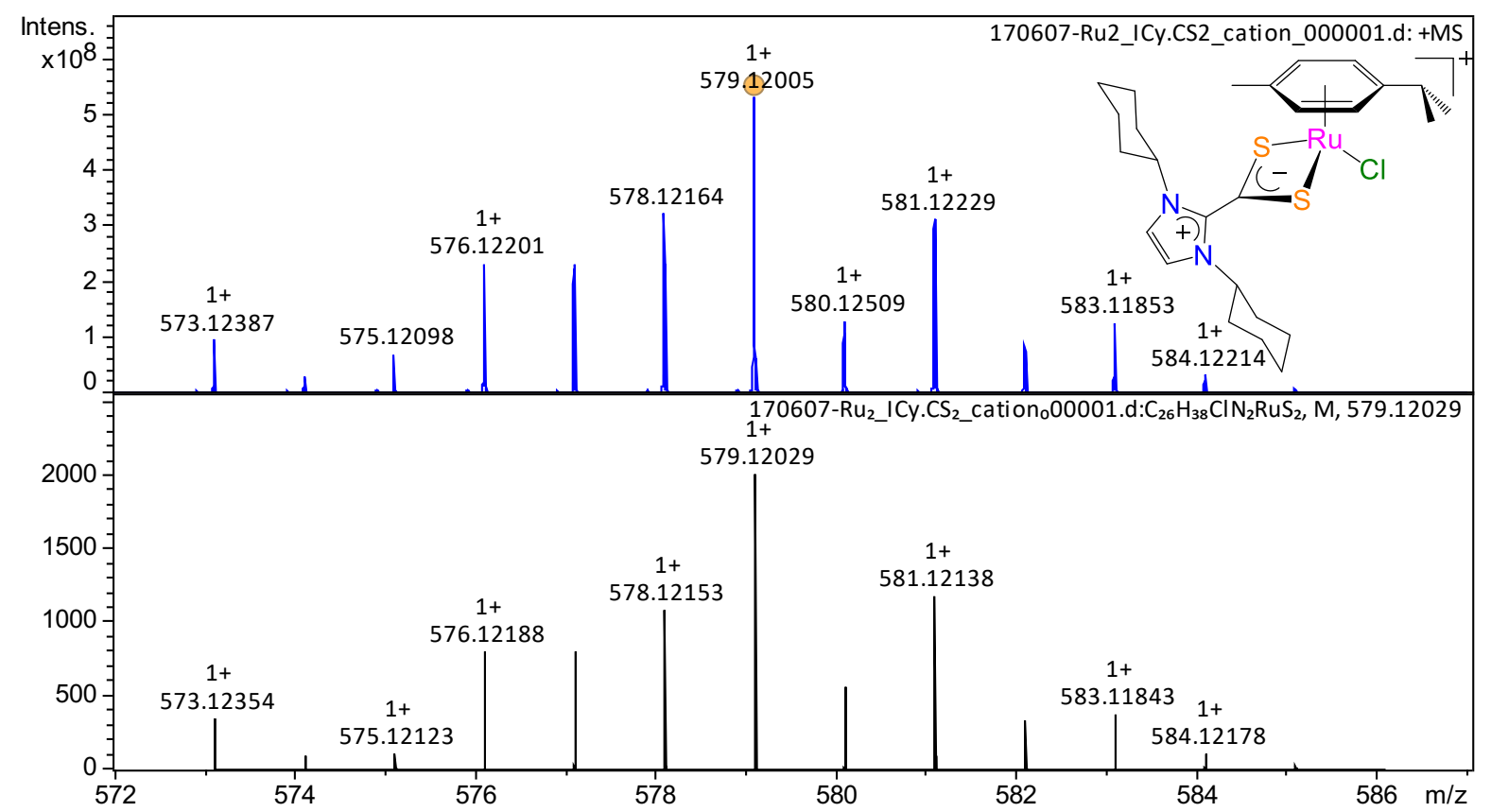

Fig. S55. HR-MS spectrum $\left(\mathrm{CH}_{3} \mathrm{CN}\right.$, positive mode $)$ of $\left[\mathrm{RuCl}(p\right.$-cymene $\left.)\left(\mathrm{S}_{2} \mathrm{C} \cdot \mathrm{ICy}\right)\right]-$ $\left[\mathrm{RuCl}_{3}(p\right.$-cymene)] (3c): isotope profile observed by ESI-MS (top) and simulated isotope pattern for the $\left[\mathrm{RuCl}(p \text {-cymene })\left(\mathrm{S}_{2} \mathrm{C} \cdot \mathrm{ICy}\right)\right]^{+}$cation (bottom)

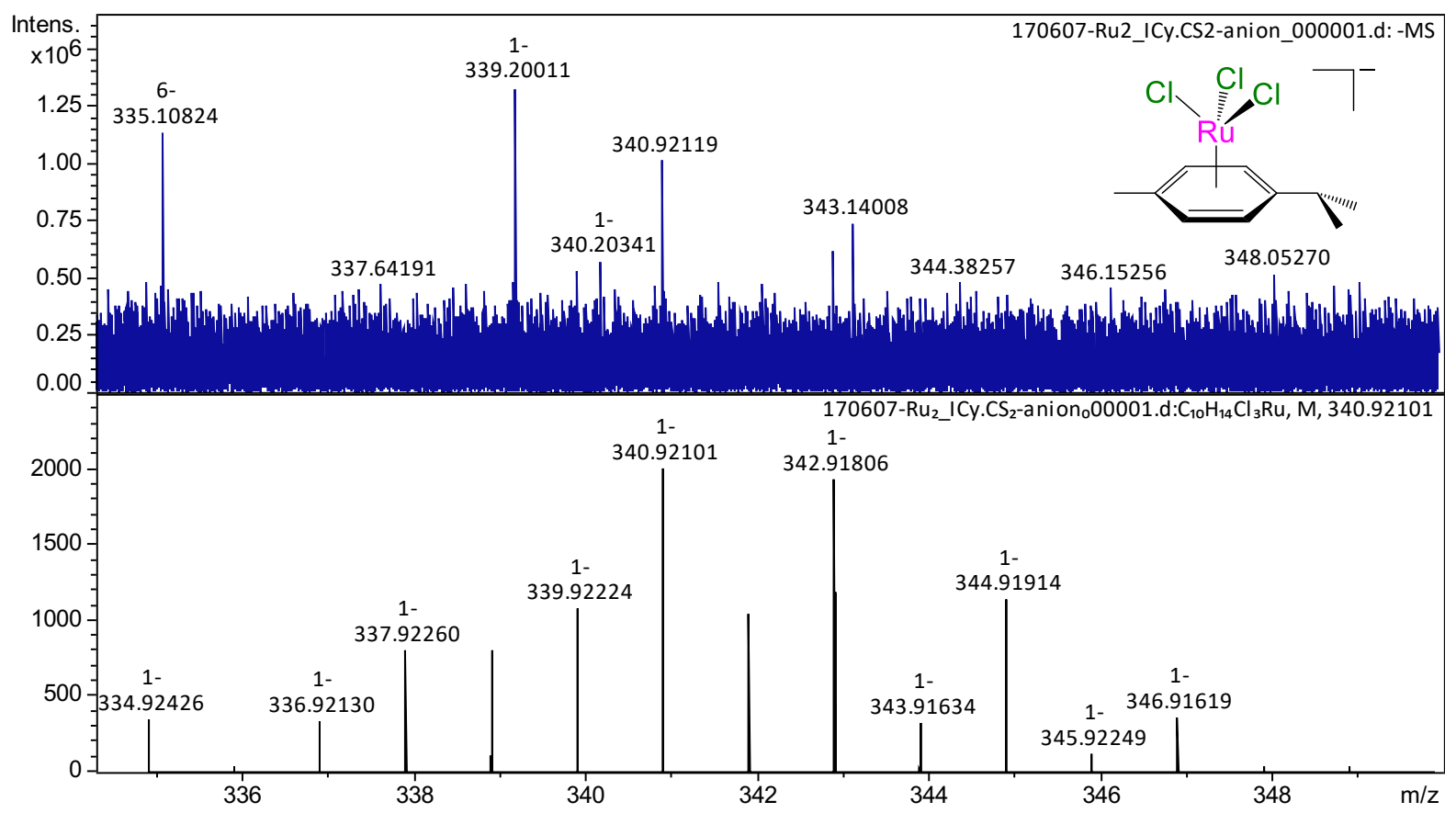

Fig. S56. HR-MS spectrum $\left(\mathrm{CH}_{3} \mathrm{CN}\right.$, negative mode $)$ of $\left[\mathrm{RuCl}(p\right.$-cymene $\left.)\left(\mathrm{S}{ }_{2} \mathrm{C} \cdot \mathrm{ICy}\right)\right]-$ $\left[\mathrm{RuCl}_{3}\right.$ (p-cymene)] (3c): isotope profile observed by ESI-MS (top) and simulated isotope pattern for the $\left[\mathrm{RuCl}_{3}(p \text {-cymene) }]^{-}\right.$anion (bottom) 


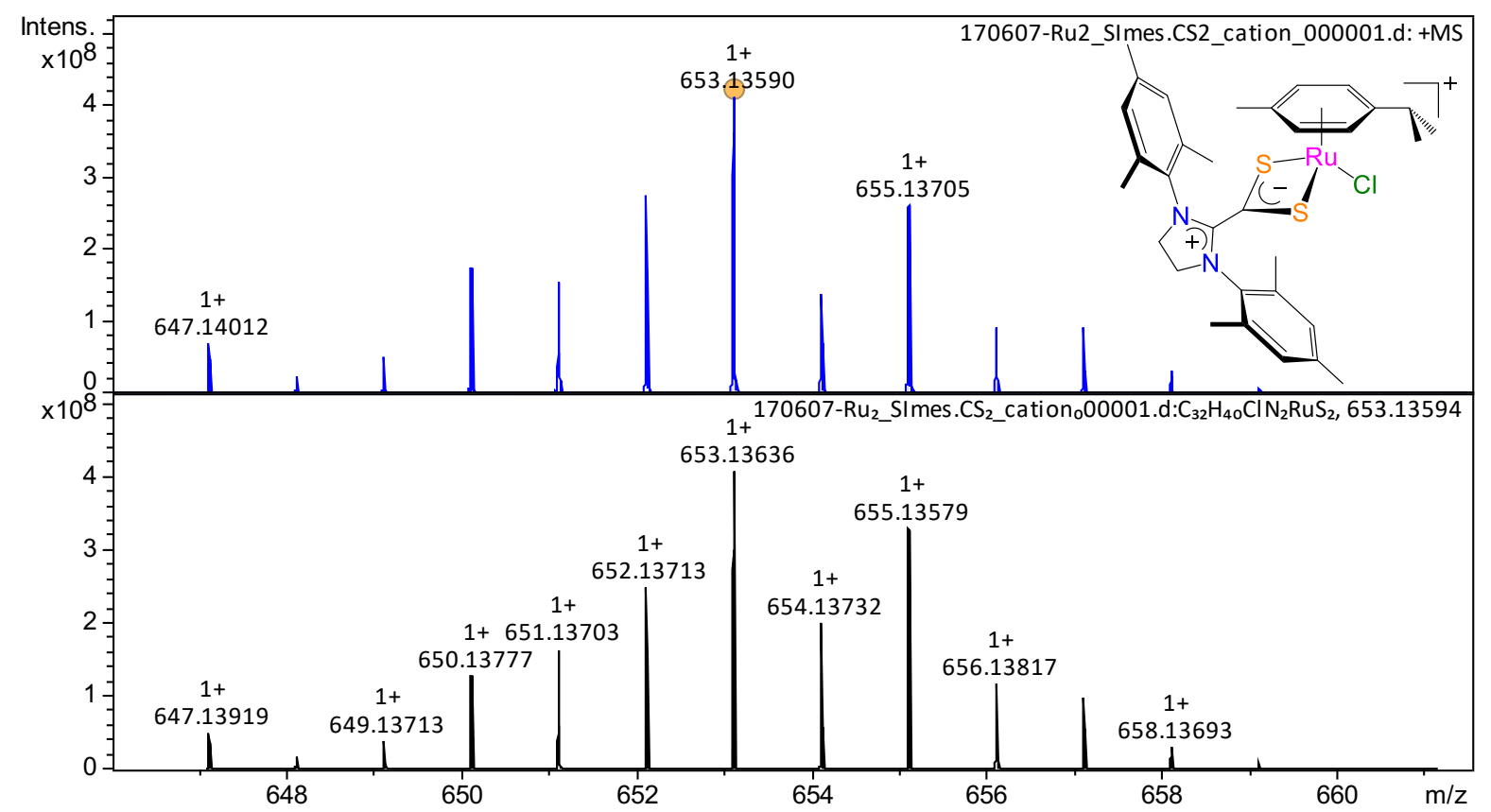

Fig. S57. HR-MS spectrum $\left(\mathrm{CH}_{3} \mathrm{CN}\right.$, positive mode $)$ of $\left[\mathrm{RuCl}(p\right.$-cymene $\left.)\left(\mathrm{S}_{2} \mathrm{C} \cdot \mathrm{SIMes}\right)\right]$ $\left[\mathrm{RuCl}_{3}(p\right.$-cymene)] (3d): isotope profile observed by ESI-MS (top) and simulated isotope pattern for the $\left[\mathrm{RuCl}(p \text {-cymene })\left(\mathrm{S}_{2} \mathrm{C} \cdot \mathrm{SIMes}\right)\right]^{+}$cation (bottom)

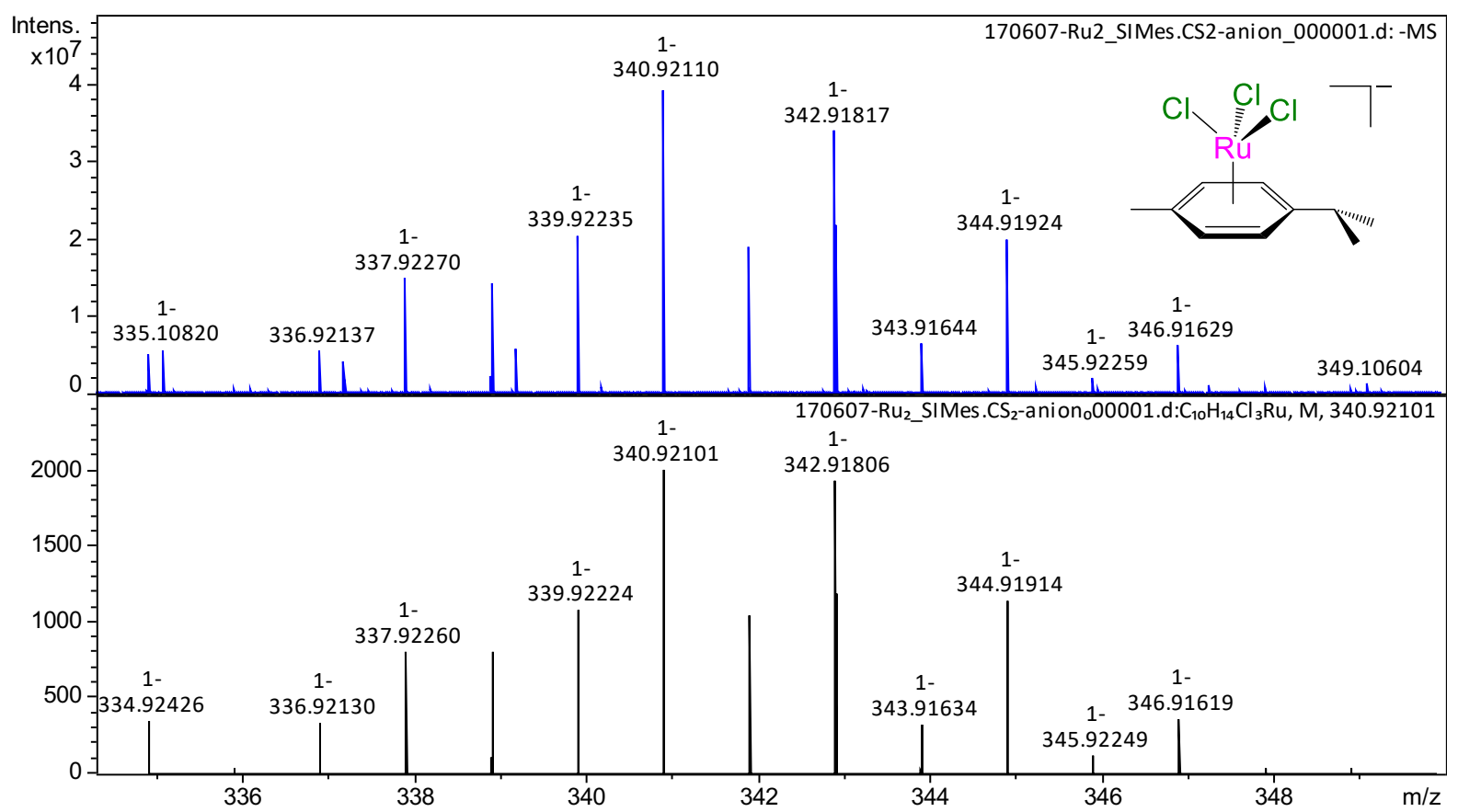

Fig. S58. HR-MS spectrum $\left(\mathrm{CH}_{3} \mathrm{CN}\right.$, negative mode $)$ of $\left[\mathrm{RuCl}(p\right.$-cymene $\left.)\left(\mathrm{S}_{2} \mathrm{C} \cdot \mathrm{SIMes}\right)\right]-$ $\left[\mathrm{RuCl}_{3}(p\right.$-cymene)] (3d): isotope profile observed by ESI-MS (top) and simulated isotope pattern for the $\left[\mathrm{RuCl}_{3}(p \text {-cymene })\right]^{-}$anion (bottom) 


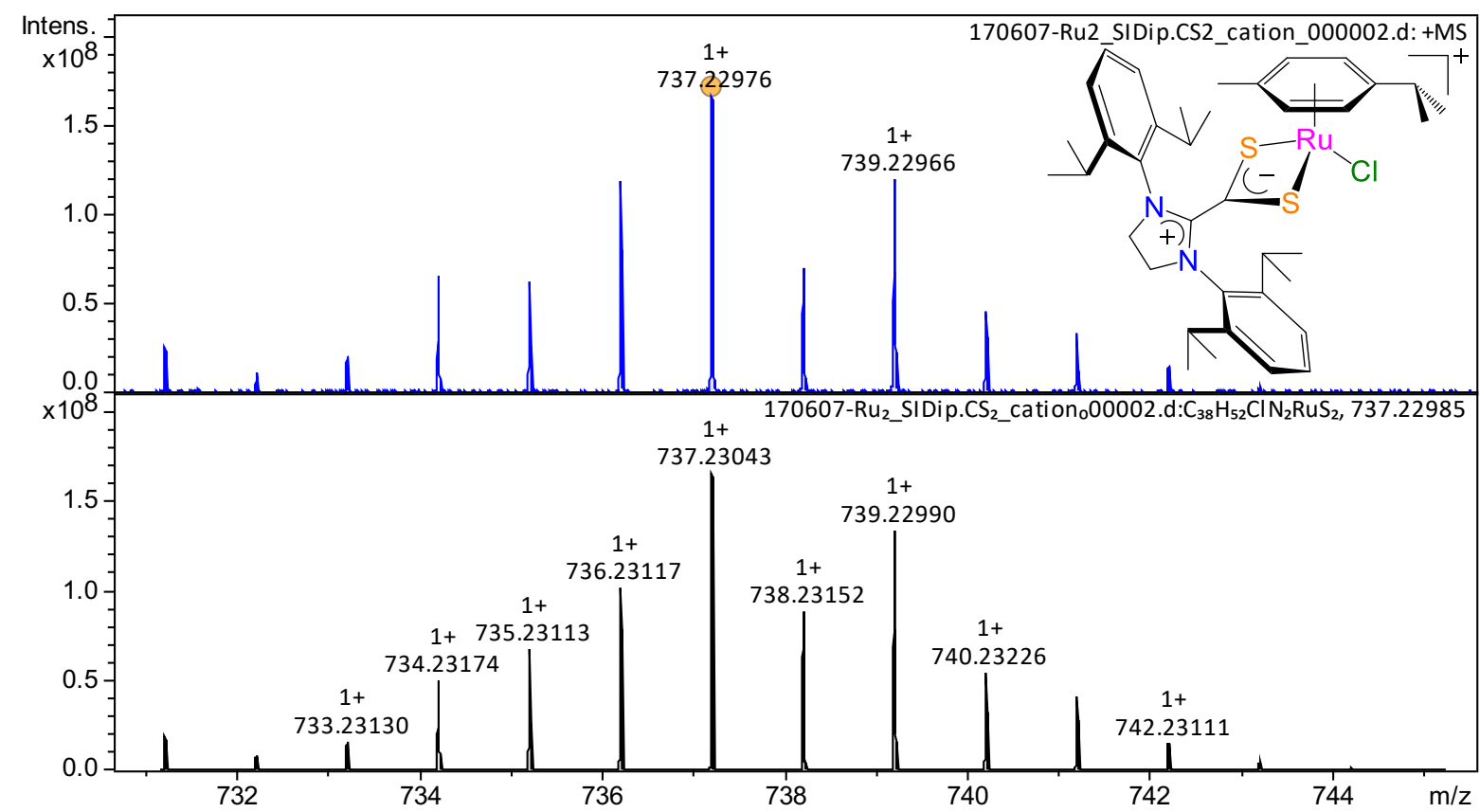

Fig. S59. HR-MS spectrum $\left(\mathrm{CH}_{3} \mathrm{CN}\right.$, positive mode $)$ of $\left[\mathrm{RuCl}(p\right.$-cymene $\left.)\left(\mathrm{S}_{2} \mathrm{C} \cdot \mathrm{SIDip}\right)\right]-$ $\left[\mathrm{RuCl}_{3}(p\right.$-cymene)] (3e): isotope profile observed by ESI-MS (top) and simulated isotope pattern for the $\left[\mathrm{RuCl}(p \text {-cymene })\left(\mathrm{S}_{2} \mathrm{C} \cdot \mathrm{SIDip}\right)\right]^{+}$cation (bottom)

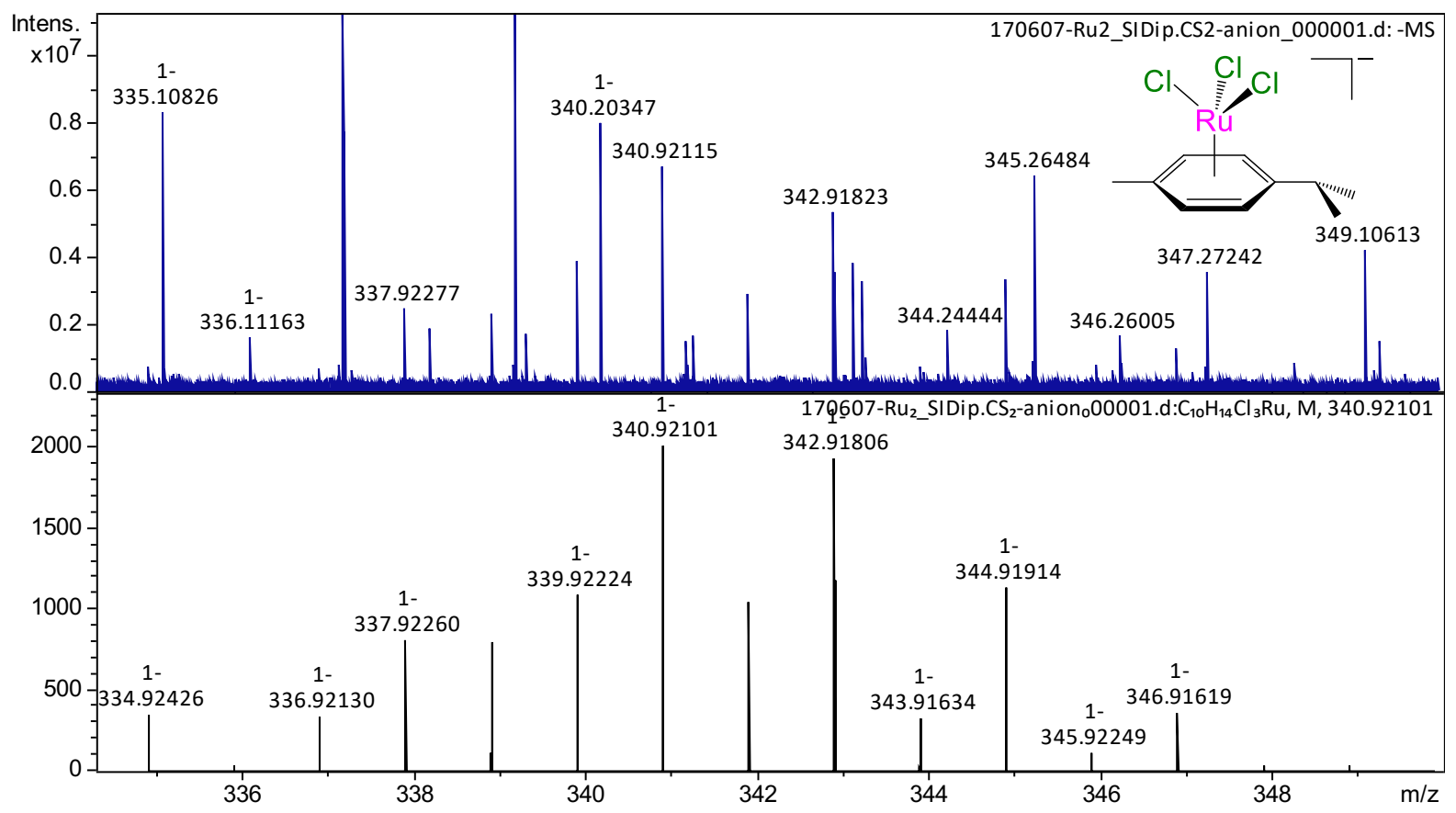

Fig. S60. HR-MS spectrum $\left(\mathrm{CH}_{3} \mathrm{CN}\right.$, negative mode $)$ of $\left[\mathrm{RuCl}(p\right.$-cymene $\left.)\left(\mathrm{S}_{2} \mathrm{C} \cdot \mathrm{SIDip}\right)\right]-$ $\left[\mathrm{RuCl}_{3}(p\right.$-cymene)] (3e): isotope profile observed by ESI-MS (top) and simulated isotope pattern for the $\left[\mathrm{RuCl}_{3}(p \text {-cymene) }]^{-}\right.$anion (bottom) 


\section{Part 5 - Crystallography}

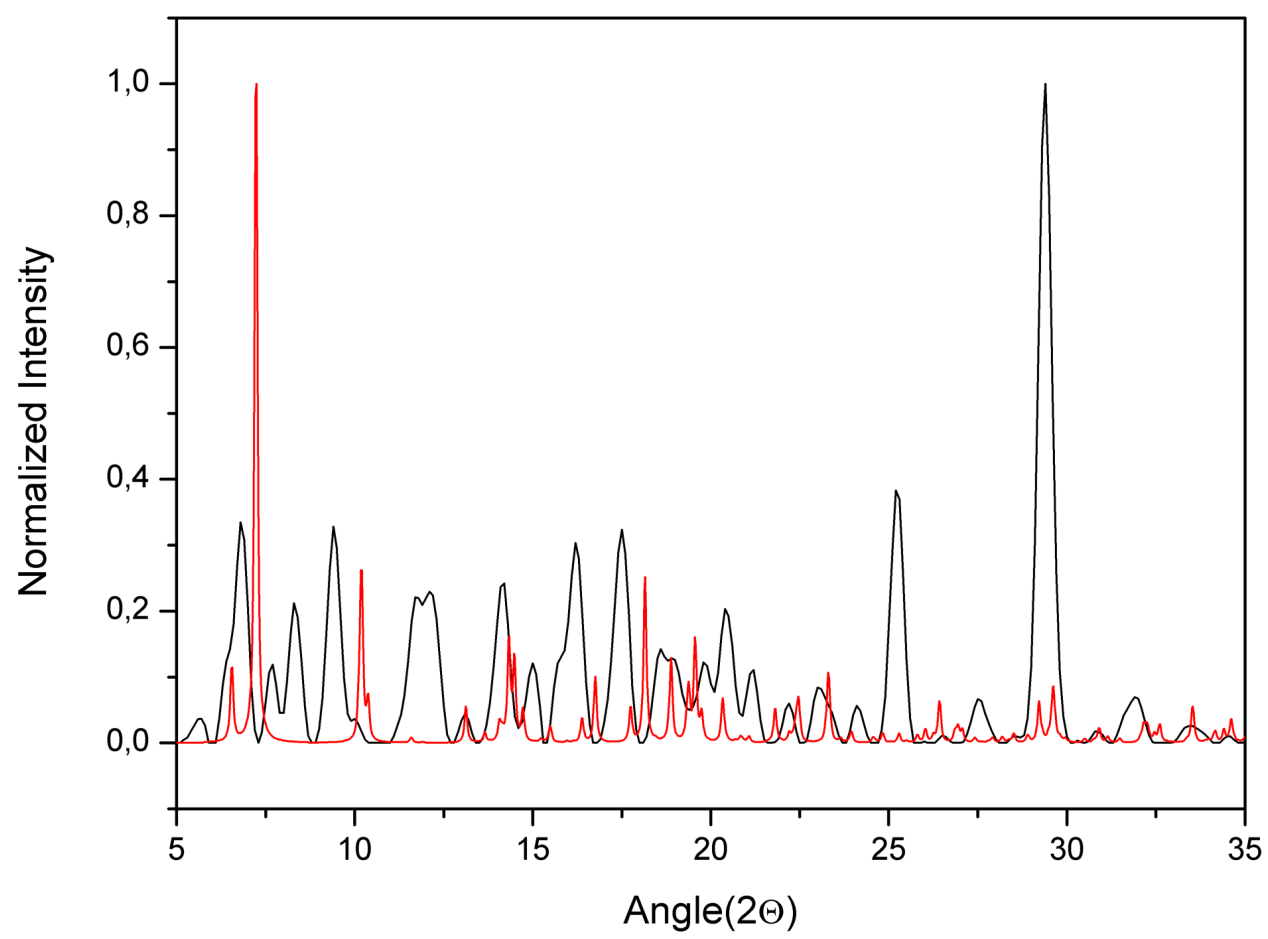

Fig. S61. Powder diffraction pattern recorded for the bulk sample obtained by crystallization of $\left[\mathrm{RuCl}(p\right.$-cymene $\left.)\left(\mathrm{S}_{2} \mathrm{C} \cdot \mathrm{ICy}\right)\right]\left[\mathrm{RuCl}_{3}(p\right.$-cymene) $)(3 \mathbf{c})$ (black curve) and simulated pattern computed for $\left[\mathrm{RuCl}(p\right.$-cymene $\left.)\left(\mathrm{S}_{2} \mathrm{C} \cdot \mathrm{ICy}\right)\right] \mathrm{Cl}\left(2(\mathbf{2 c}) \cdot 9.75\left(\mathrm{H}_{2} \mathrm{O}\right)\right)($ red curve $)$

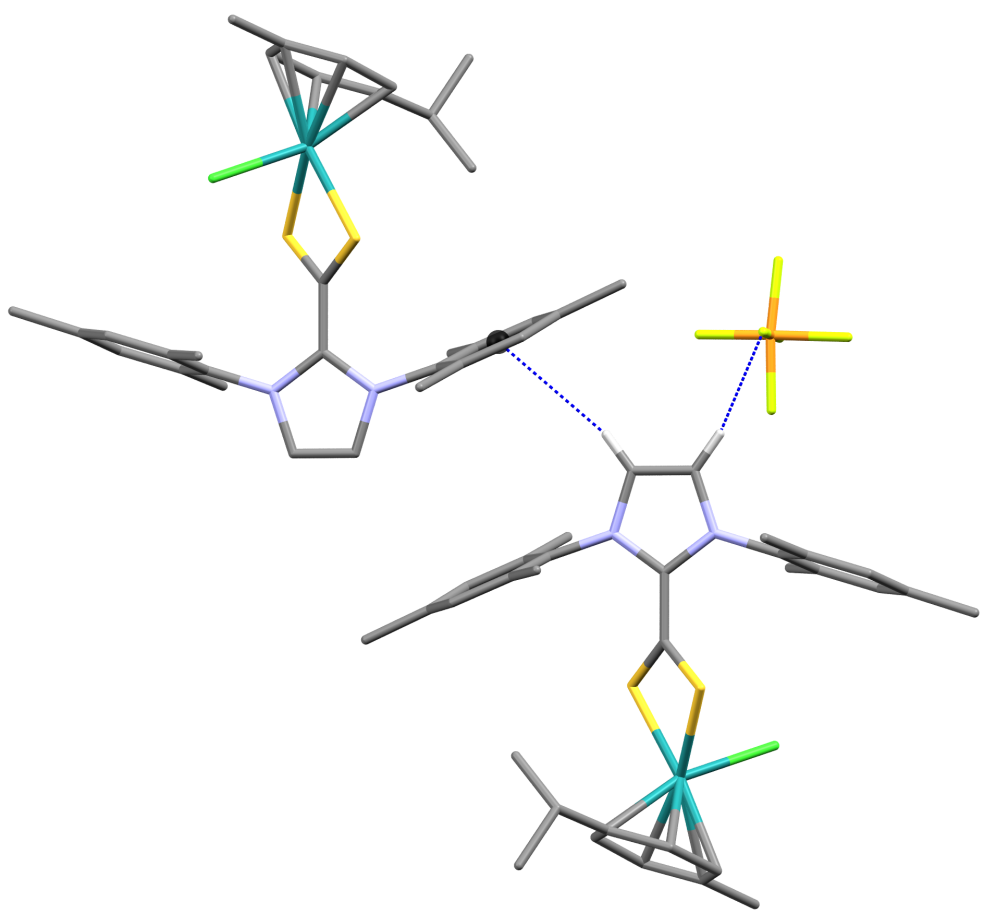

Fig. S62. Molecular structure of $\left[\mathrm{RuCl}(p\right.$-cymene $\left.)\left(\mathrm{S}_{2} \mathrm{C} \cdot \mathrm{IMes}\right)\right] \mathrm{PF}_{6}(\mathbf{1 a})$ showing the $\mathrm{H}$-bonds of the imidazolium protons (hydrogen atoms were omitted except those directly bound to the heterocyclic ring) 


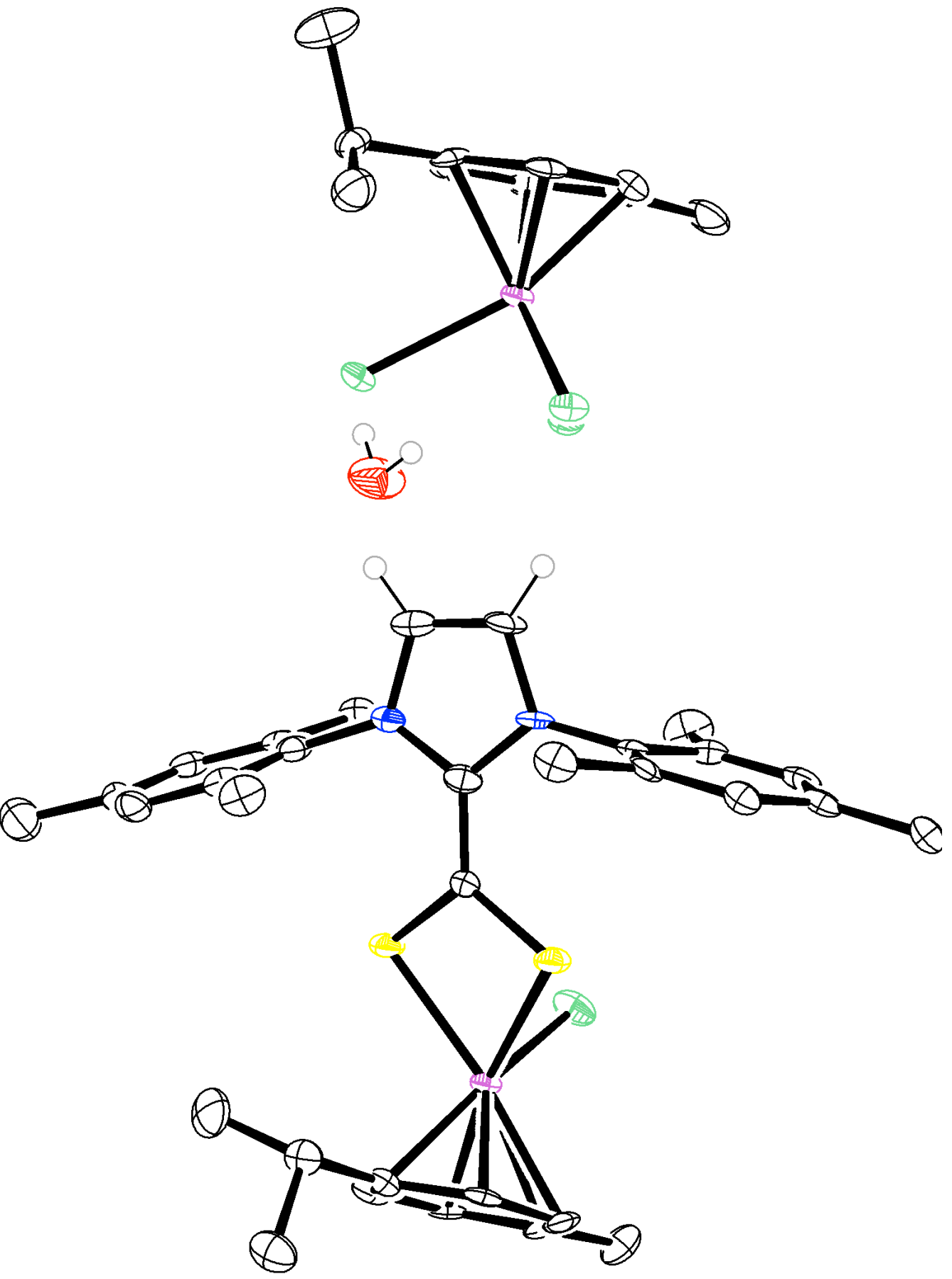

Fig. S63. ORTEP representation of $\left[\mathrm{RuCl}(p\right.$-cymene $\left.)\left(\mathrm{S}_{2} \mathrm{C} \cdot \mathrm{IMes}\right)\right]\left[\mathrm{RuCl}_{3}(p\right.$-cymene $\left.)\right]$ cocrystallized with water $\left(\mathbf{3} \mathbf{a} \cdot \mathrm{H}_{2} \mathrm{O}\right)$. Hydrogen atoms were omitted except those directly bound to the heterocyclic ring and oxygen. Thermal ellipsoids were drawn at the $50 \%$ probability level 


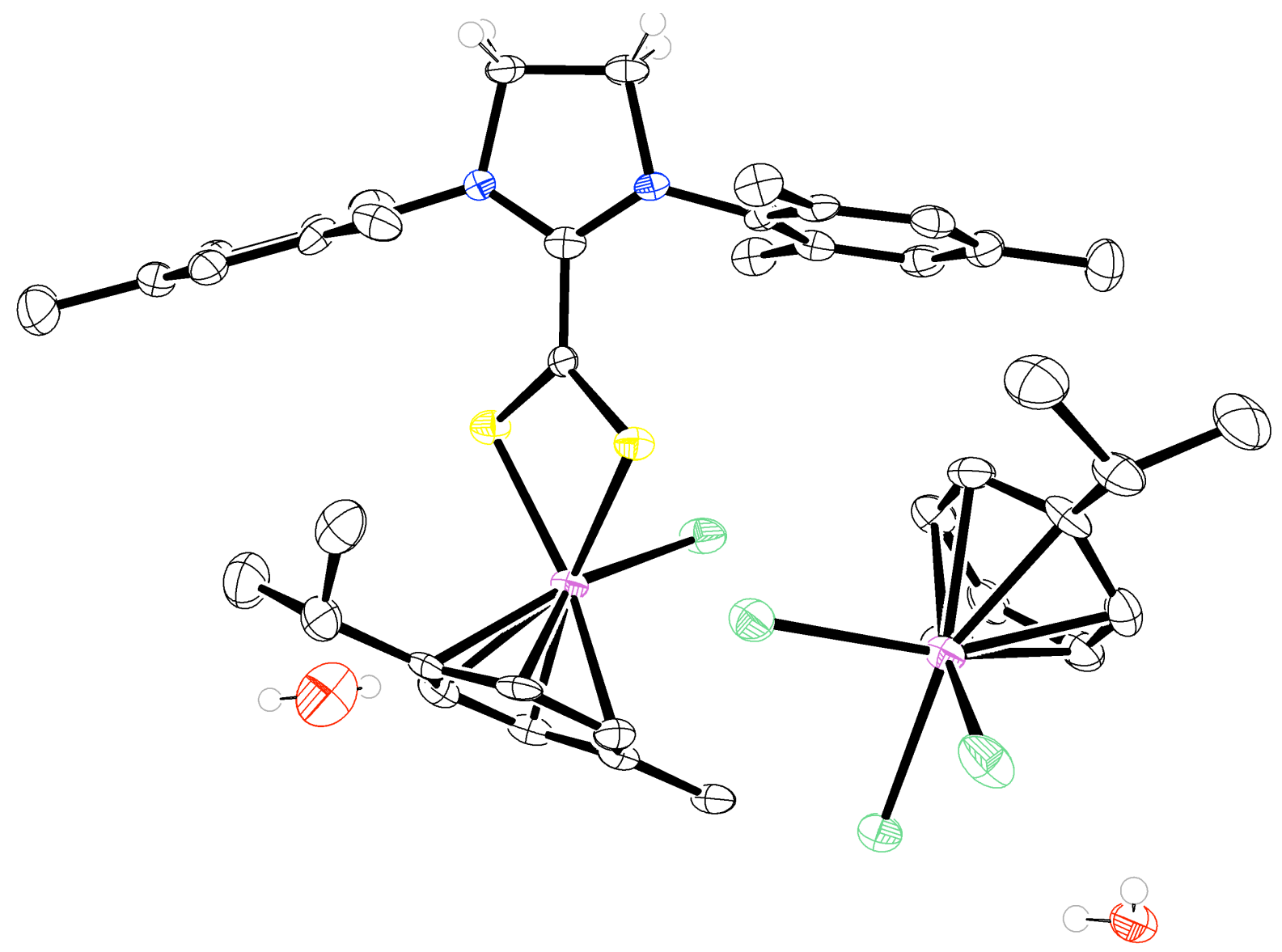

Fig. S64. ORTEP representation of $\left[\mathrm{RuCl}(p\right.$-cymene $\left.)\left(\mathrm{S}_{2} \mathrm{C} \cdot \mathrm{SIMes}\right)\right]\left[\mathrm{RuCl}_{3}(p\right.$-cymene $\left.)\right]$ cocrystallized with water $\left(2(\mathbf{3 d}) \cdot 3\left(\mathrm{H}_{2} \mathrm{O}\right)\right)$. Hydrogen atoms were omitted except those directly bound to the heterocyclic ring and oxygen. Thermal ellipsoids were drawn at the $50 \%$ probability level 


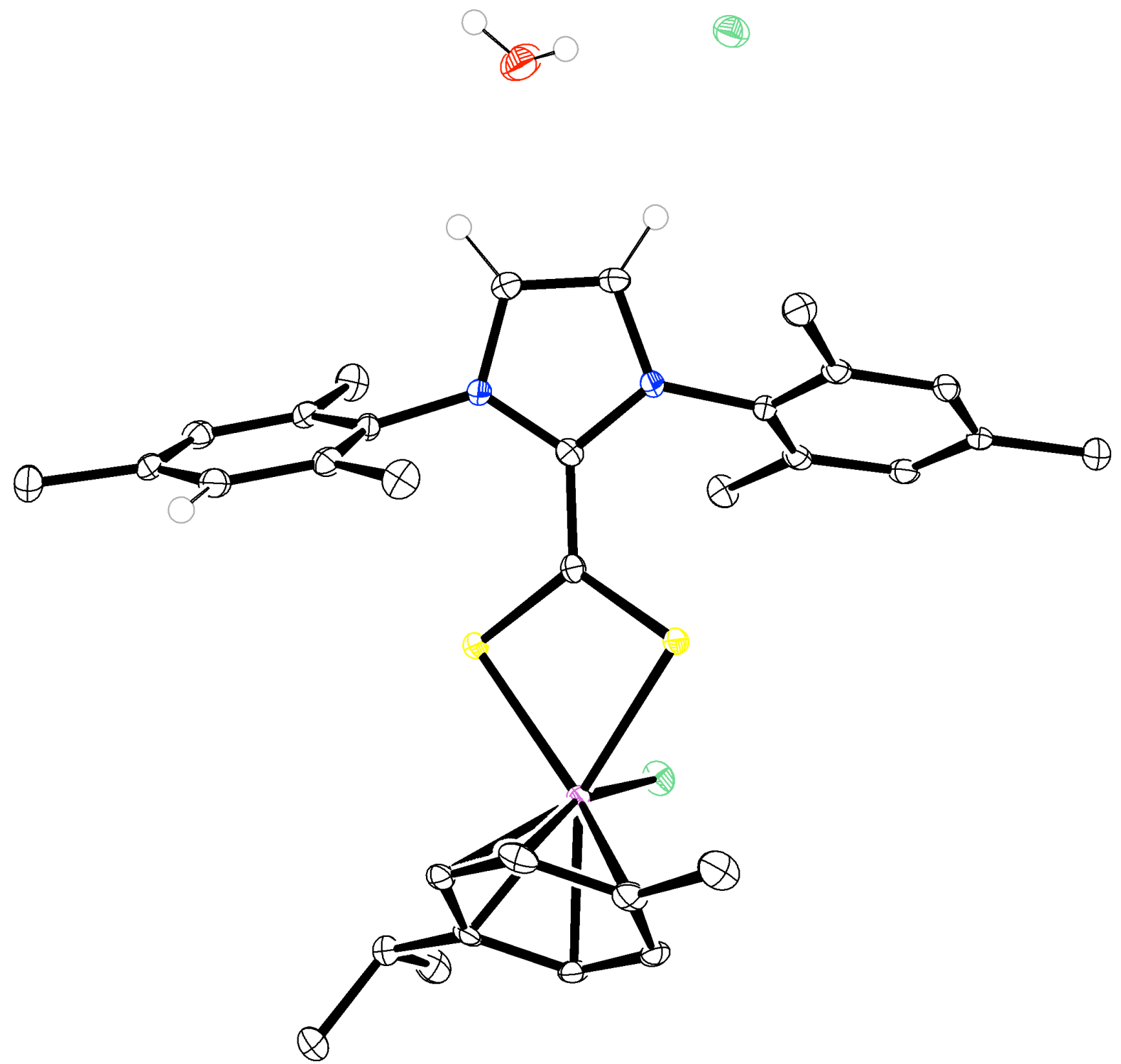

Fig. S65. ORTEP representation of $\left[\mathrm{RuCl}(p\right.$-cymene $\left.)\left(\mathrm{S}_{2} \mathrm{C} \cdot \mathrm{IMes}\right)\right] \mathrm{Cl}$ co-crystallized with water $\left(\mathbf{2} \mathbf{a} \cdot \mathrm{H}_{2} \mathrm{O}\right)$. Hydrogen atoms were omitted except those directly bound to the heterocyclic ring and oxygen. Thermal ellipsoids were drawn at the 50\% probability level 


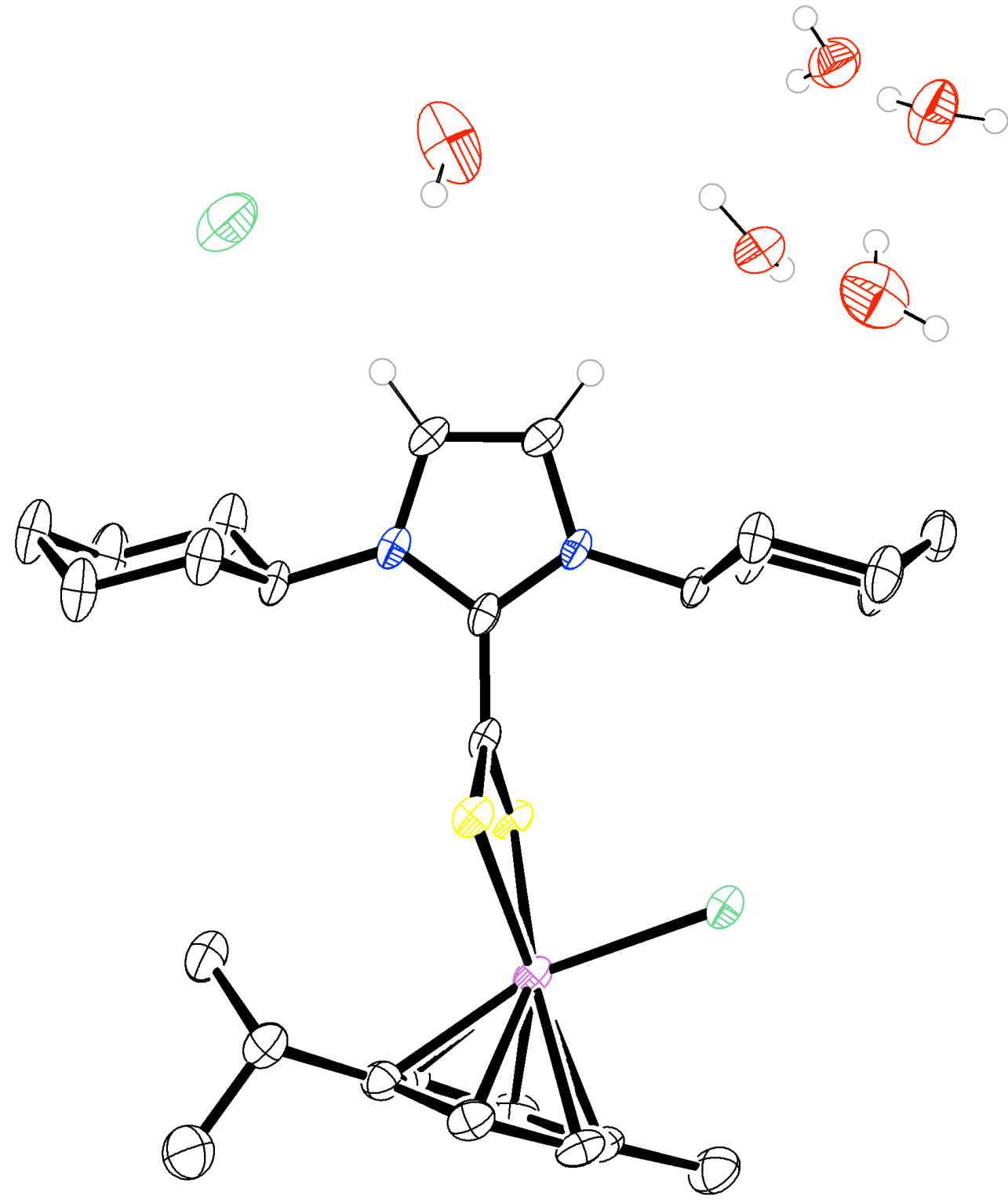

Fig. S66. ORTEP representation of $\left[\mathrm{RuCl}(p\right.$-cymene $\left.)\left(\mathrm{S}_{2} \mathrm{C} \cdot \mathrm{ICy}\right)\right] \mathrm{Cl}$ co-crystallized with water $\left(2(2 \mathbf{c}) \cdot 9.75\left(\mathrm{H}_{2} \mathrm{O}\right)\right)$. Hydrogen atoms were omitted except those directly bound to the heterocyclic ring and oxygen. Thermal ellipsoids were drawn at the 50\% probability level 
Table S1. Analysis of Short Ring-Interactions with Cg-Cg Distances ( $)$, and Angles (deg) in $\left[\mathrm{RuCl}(p\right.$-cymene $\left.)\left(\mathrm{S}_{2} \mathrm{C} \cdot \mathrm{IMes}\right)\right] \mathrm{Cl}(\mathbf{2 a}),\left[\mathrm{RuCl}(p\right.$-cymene $\left.)\left(\mathrm{S}_{2} \mathrm{C} \cdot \mathrm{IMes}\right)\right]\left[\mathrm{RuCl}_{3}(p\right.$-cymene $\left.)\right]$ (3a), and $\left[\mathrm{RuCl}(p\right.$-cymene $\left.)\left(\mathrm{S}_{2} \mathrm{C} \cdot \mathrm{SIMes}\right)\right]\left[\mathrm{RuCl}_{3}(p\right.$-cymene) $)(3 \mathbf{d})$. Reciprocal interactions were omitted.

\begin{tabular}{|c|c|c|c|c|c|c|c|}
\hline Complex & $\mathrm{Cg}(\mathrm{I}) \rightarrow \mathrm{Cg}(\mathrm{J})$ & $\mathrm{Cg}-\mathrm{Cg}$ & Alpha & Beta & Gamma & CgI_Perp & CgJ_Perp \\
\hline \multirow{6}{*}{$\mathbf{2} \mathbf{a} \cdot \mathrm{H}_{2} \mathrm{O}$} & $\operatorname{Cg}(1) \rightarrow \operatorname{Cg}(2)^{i}$ & $5.8119(13)$ & $72.60(11)$ & 20.4 & 52.4 & $3.5458(9)$ & $5.4472(9)$ \\
\hline & $\mathrm{Cg}(1) \rightarrow \mathrm{Cg}(4)^{\mathrm{ii}}$ & $4.8790(13)$ & $61.22(11)$ & 46.3 & 34.0 & $4.0465(9)$ & $3.3729(9)$ \\
\hline & $\mathrm{Cg}(2) \rightarrow \mathrm{Cg}(3)^{\mathrm{iii}}$ & $5.2461(13)$ & $44.17(10)$ & 34.7 & 53.0 & $3.1572(8)$ & $4.3107(9)$ \\
\hline & $\mathrm{Cg}(3) \rightarrow \mathrm{Cg}(3)^{\mathrm{iv}}$ & $5.5539(13)$ & $0.00(10)$ & 54.0 & 54.0 & $3.2646(8)$ & $3.2646(8)$ \\
\hline & $\mathrm{Cg}(3) \rightarrow \mathrm{Cg}(4)^{\mathrm{ii}}$ & $4.3400(12)$ & $11.71(10)$ & 31.9 & 34.7 & $3.5681(8)$ & $3.6840(8)$ \\
\hline & $\mathrm{Cg}(4) \rightarrow \mathrm{Cg}(1)^{\mathrm{v}}$ & $4.8790(13)$ & $61.22(11)$ & 34.0 & 46.3 & $3.3729(9)$ & $4.0465(9)$ \\
\hline \multirow{5}{*}{$\mathbf{3 a} \cdot \mathrm{H}_{2} \mathrm{O}$} & $\mathrm{Cg}(1) \rightarrow \mathrm{Cg}(2)^{\mathrm{vi}}$ & $5.681(4)$ & $79.9(3)$ & 43.7 & 53.4 & $3.383(2)$ & $4.106(3)$ \\
\hline & $\mathrm{Cg}(2) \rightarrow \mathrm{Cg}(2)^{\mathrm{vi}}$ & $5.009(3)$ & $0.0(3)$ & 45.0 & 45.0 & $3.543(2)$ & $3.543(2)$ \\
\hline & $\mathrm{Cg}(2) \rightarrow \mathrm{Cg}(5)^{\mathrm{ix}}$ & $5.626(4)$ & $27.2(3)$ & 59.8 & 57.7 & $3.009(3)$ & $2.831(2)$ \\
\hline & $\mathrm{Cg}(3) \rightarrow \mathrm{Cg}(4)^{\mathrm{vii}}$ & $4.233(4)$ & $22.0(3)$ & 26.8 & 5.8 & $4.212(3)$ & $3.777(2)$ \\
\hline & $\mathrm{Cg}(3) \rightarrow \mathrm{Cg}(5)^{\text {viii }}$ & $5.053(4)$ & 11.1(3) & 44.3 & 51.3 & $3.159(2)$ & $3.618(3)$ \\
\hline \multirow{4}{*}{$\begin{array}{l}2(3 \mathbf{3 d}) \\
\cdot 3 \mathrm{H}_{2} \mathrm{O}\end{array}$} & $\mathrm{Cg}(3) \rightarrow \mathrm{Cg}(4)^{\mathrm{xii}}$ & $3.918(4)$ & $11.0(3)$ & 29.2 & 19.8 & $3.685(2)$ & $3.419(2)$ \\
\hline & $\mathrm{Cg}(3) \rightarrow \operatorname{Cg}(5)^{x}$ & $4.756(4)$ & $39.2(3)$ & 34.0 & 61.7 & $2.252(2)$ & $3.944(2)$ \\
\hline & $\mathrm{Cg}(4) \rightarrow \mathrm{Cg}(1)^{\mathrm{xi}}$ & $5.720(4)$ & $68.5(3)$ & 57.3 & 62.5 & $2.644(3)$ & $3.091(3)$ \\
\hline & $\mathrm{Cg}(5) \rightarrow \mathrm{Cg}(2)^{\mathrm{xii}}$ & $5.783(4)$ & $39.3(3)$ & 42.0 & 67.8 & $2.182(3)$ & $4.300(2)$ \\
\hline
\end{tabular}

$\mathrm{Cg}(1): \mathrm{C} 2 \mathrm{~N} 1 \mathrm{C} 3 \mathrm{C} 4 \mathrm{~N} 2 ; \mathrm{Cg}(2): \mathrm{C} 5 \rightarrow \mathrm{C} 10 ; \mathrm{Cg}(3): \mathrm{C} 14 \rightarrow \mathrm{C} 19 ; \mathrm{Cg}(4): \mathrm{C} 23 \rightarrow \mathrm{C} 28$;

$\mathrm{Cg}(5): \mathrm{C} 33 \rightarrow \mathrm{C} 38$

Symmetry codes: i) $1-\mathrm{X}, 1-\mathrm{Y},-\mathrm{Z}$; ii) $1+\mathrm{X}, \mathrm{Y}, \mathrm{Z}$; iii) $\mathrm{X},-1+\mathrm{Y}, \mathrm{Z}$; iv) $1-\mathrm{X}, 2-\mathrm{Y},-\mathrm{Z}$;

v) $-1+\mathrm{X}, \mathrm{Y}, \mathrm{Z}$; vi) $2-\mathrm{X},-\mathrm{Y}, 1-\mathrm{Z}$; vii) $1-\mathrm{X},-\mathrm{Y}, 1-\mathrm{Z}$; vii) $-1 / 2+\mathrm{X}, 1 / 2-\mathrm{Y}, 1 / 2+\mathrm{Z}$;

viii) $1 / 2+X, 1 / 2-Y,-1 / 2+Z$; ix) $1-X,-Y, 1-Z$; x $)-1 / 2+X, Y, 3 / 2-Z$;

xi) $1 / 2+\mathrm{X}, \mathrm{Y}, 3 / 2-\mathrm{Z}$; xii) $\mathrm{X}, 3 / 2-\mathrm{Y},-1 / 2+\mathrm{Z}$ 
Table S2. Selected Bond Lengths ( $\AA$ ), and Angles (deg) for the Hydrogen Bonds Between the Counteranions and Solvent molecules Derived from the Crystal Structures of $\left[\operatorname{RuCl}(p\right.$-cymene $\left.)\left(\mathrm{S}_{2} \mathrm{C} \cdot \mathrm{IMes}\right)\right] \mathrm{Cl}(\mathbf{2 a}),\left[\mathrm{RuCl}(p\right.$-cymene $\left.)\left(\mathrm{S}_{2} \mathrm{C} \cdot \mathrm{IMes}\right)\right]\left[\mathrm{RuCl}_{3}(p\right.$-cymene $\left.)\right](3 \mathbf{a})$, $\left[\mathrm{RuCl}(p\right.$-cymene $\left.)\left(\mathrm{S}_{2} \mathrm{C} \cdot \mathrm{ICy}\right)\right] \mathrm{Cl}(\mathbf{2 c})$, and $\left[\mathrm{RuCl}(p\right.$-cymene $\left.)\left(\mathrm{S}_{2} \mathrm{C} \cdot \mathrm{SIMes}\right)\right]\left[\mathrm{RuCl}_{3}(p\right.$-cymene $\left.)\right]$ (3d).

\begin{tabular}{|c|c|c|c|c|c|}
\hline Complex & Donor-H $\cdots$ Acceptor & $\mathrm{D}-\mathrm{H}$ & $\mathrm{H} \cdots \mathrm{A}$ & $\mathrm{D} \cdots \mathrm{A}$ & $\mathrm{D}-\mathrm{H} \cdots \mathrm{A}$ \\
\hline \multirow{2}{*}{$\mathbf{2 a} \cdot \mathrm{H}_{2} \mathrm{O}$} & $\mathrm{O} 1-\mathrm{H} 1 \mathrm{O} \cdots \mathrm{Cl} 2$ & $0.75(3)$ & $2.50(4)$ & $3.244(2)$ & $171(3)$ \\
\hline & $\mathrm{O} 1-\mathrm{H} 2 \mathrm{O} \cdots \mathrm{Cl} 2^{\mathrm{i}}$ & $0.81(4)$ & $2.40(4)$ & $3.205(2)$ & 170(3) \\
\hline \multirow[t]{2}{*}{$3 \mathbf{a} \cdot \mathrm{H}_{2} \mathrm{O}$} & $\mathrm{O} 1-\mathrm{H} 1 \mathrm{O} \cdots \mathrm{Cl} 3$ & $0.82(2)$ & $2.51(4)$ & $3.275(5)$ & 156(7) \\
\hline & $\mathrm{O} 1-\mathrm{H} 2 \mathrm{O} \cdots \mathrm{Cl} 4^{\mathrm{ii}}$ & $0.83(2)$ & $2.54(3)$ & $3.323(5)$ & $157(6)$ \\
\hline \multirow[t]{9}{*}{$2(2 \mathrm{c}) \cdot 9.75\left(\mathrm{H}_{2} \mathrm{O}\right)$} & $\mathrm{O} 1 \mathrm{~W}-\mathrm{H} 1 \mathrm{O} . \mathrm{Cl} 2$ & $0.843(7)$ & $2.65(7)$ & $3.169(4)$ & 121(6) \\
\hline & $\mathrm{O} 2 \mathrm{~W}-\mathrm{H} 21 \mathrm{O} \cdots \mathrm{Cl} 2^{\mathrm{iii}}$ & $0.847(10)$ & $2.38(3)$ & $3.157(4)$ & $152(6)$ \\
\hline & $\mathrm{O} 2 \mathrm{~W}-\mathrm{H} 22 \mathrm{O} \cdots \mathrm{O} 5 \mathrm{~W}$ & $0.848(10)$ & $2.06(3)$ & $2.878(12)$ & 162(7) \\
\hline & $\mathrm{O} 3 \mathrm{~W}-\mathrm{H} 31 \mathrm{O} \cdots \mathrm{O} 4 \mathrm{~W}$ & $0.848(10)$ & $2.19(3)$ & $2.990(8)$ & $158(6)$ \\
\hline & $\mathrm{O} 3 \mathrm{~W}-\mathrm{H} 32 \mathrm{O} \cdots \mathrm{O} 2 \mathrm{~W}^{\mathrm{iv}}$ & $0.846(10)$ & $2.07(2)$ & $2.903(8)$ & 166(9) \\
\hline & $\mathrm{O} 4 \mathrm{~W}-\mathrm{H} 41 \mathrm{O} \cdots \mathrm{Cl1}^{\mathrm{v}}$ & $0.846(10)$ & $2.55(2)$ & $3.342(3)$ & $157(4)$ \\
\hline & $\mathrm{O} 4 \mathrm{~W}-\mathrm{H} 42 \mathrm{O} \cdots \mathrm{Cl} 2^{\mathrm{vi}}$ & $0.850(10)$ & $2.333(17)$ & $3.165(4)$ & $166(5)$ \\
\hline & $\mathrm{O} 5 \mathrm{~W}-\mathrm{H} 51 \mathrm{O} \cdots \mathrm{O} 3 \mathrm{~W}$ & $0.849(10)$ & $2.13(3)$ & $2.943(14)$ & 160(7) \\
\hline & $\mathrm{O} 5 \mathrm{~W}-\mathrm{H} 52 \mathrm{O} \cdots \mathrm{O} 4 \mathrm{~W}^{\mathrm{vii}}$ & $0.855(10)$ & $2.31(5)$ & $3.027(11)$ & 141(7) \\
\hline \multirow[t]{4}{*}{$2(3 \mathbf{d}) \cdot 3 \mathrm{H}_{2} \mathrm{O}$} & $\mathrm{O} 1-\mathrm{H} 11 \mathrm{O} \cdots \mathrm{Cl} 4^{\text {viii }}$ & $0.83(2)$ & $2.35(3)$ & $3.176(7)$ & 172(9) \\
\hline & $\mathrm{O} 1-\mathrm{H} 12 \mathrm{O} \cdots \mathrm{Cl} 4^{\mathrm{ix}}$ & $0.82(2)$ & $2.49(4)$ & $3.266(6)$ & $157(9)$ \\
\hline & $\mathrm{O} 2-\mathrm{H} 21 \mathrm{O} \cdots \mathrm{Cl} 3$ & $0.82(2)$ & $2.54(8)$ & $3.213(10)$ & 139(10) \\
\hline & $\mathrm{O} 2-\mathrm{H} 22 \mathrm{O} \cdots \mathrm{Cl} 4$ & $0.82(2)$ & $2.80(7)$ & $3.481(10)$ & $142(9)$ \\
\hline
\end{tabular}

Symmetry codes: i) $2-\mathrm{x}, 1-\mathrm{y},-\mathrm{z}$; ii) $1-\mathrm{x},-\mathrm{y}, 1-\mathrm{z}$; iii) $1-\mathrm{x}, \mathrm{y}, 3 / 2-\mathrm{z}$; iv) $1-\mathrm{x}, \mathrm{y}, 3 / 2-$ $\mathrm{z}$; v) $\mathrm{x}, 1-\mathrm{y}, 1 / 2+\mathrm{z}$; vi) $1-\mathrm{x}, 1+\mathrm{y}, 3 / 2-\mathrm{z}$; vii) $3 / 2-\mathrm{x},-1 / 2+\mathrm{y}, 3 / 2-\mathrm{z}$; viii) $\mathrm{x}, 3 / 2-\mathrm{y}$, $1 / 2+\mathrm{z}$; iv) $2-\mathrm{x},-1 / 2+\mathrm{y}, 3 / 2-\mathrm{z}$ 


\section{Part 6 - Cytotoxicity}
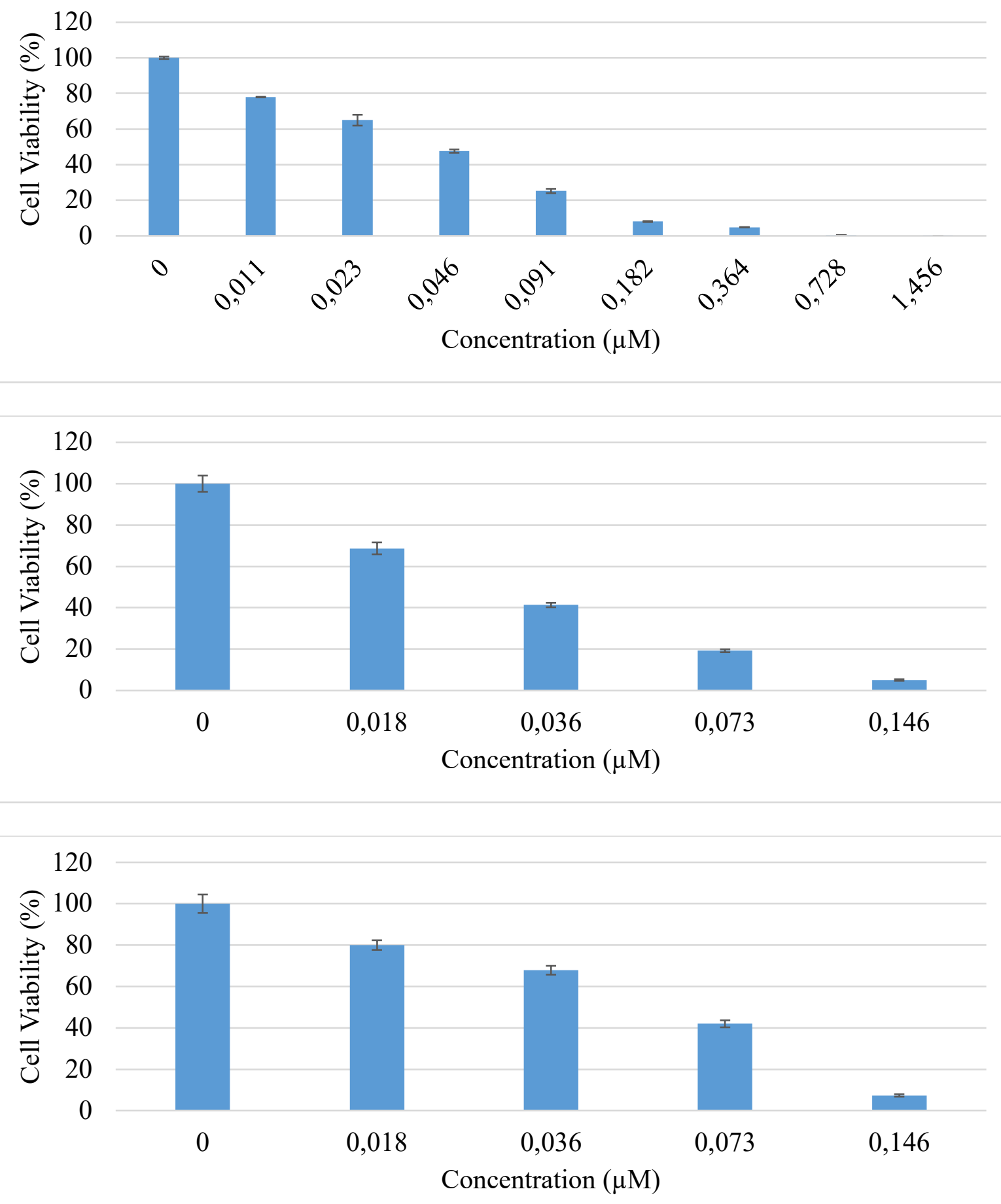

Fig. S67. In vitro cytotoxicity of $\left[\mathrm{RuCl}(p\right.$-cymene $\left.)\left(\mathrm{S}_{2} \mathrm{C} \cdot \mathrm{IMes}\right)\right] \mathrm{Cl}(\mathbf{2 a})$ against $\mathrm{K} 562$ cells measured after $72 \mathrm{~h}$. 

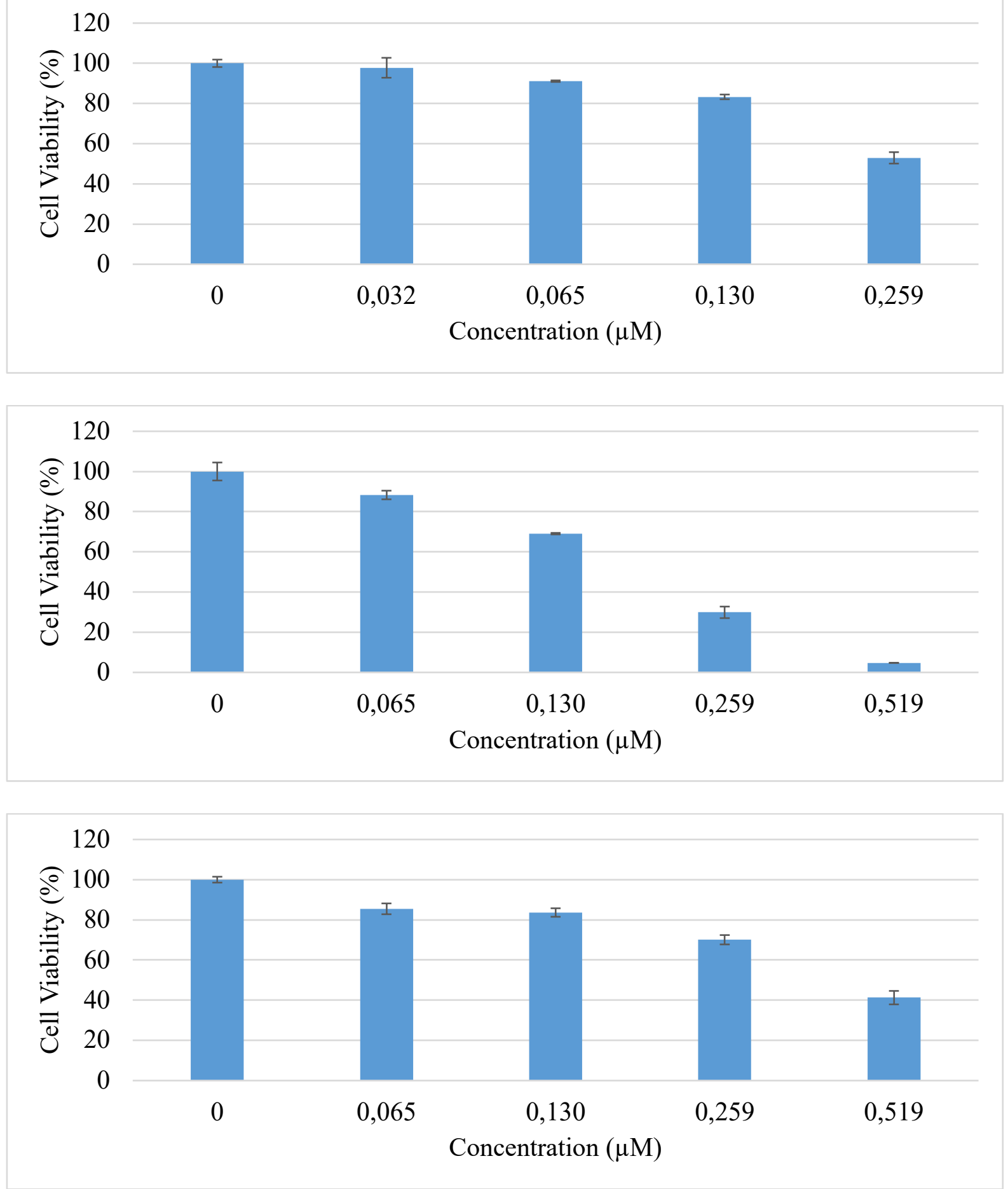

Fig. S68. In vitro cytotoxicity of $\left[\mathrm{RuCl}(p\right.$-cymene $\left.)\left(\mathrm{S}_{2} \mathrm{C} \cdot \mathrm{IDip}\right)\right] \mathrm{Cl}(\mathbf{2 b})$ against $\mathrm{K} 562$ cells measured after $72 \mathrm{~h}$. 

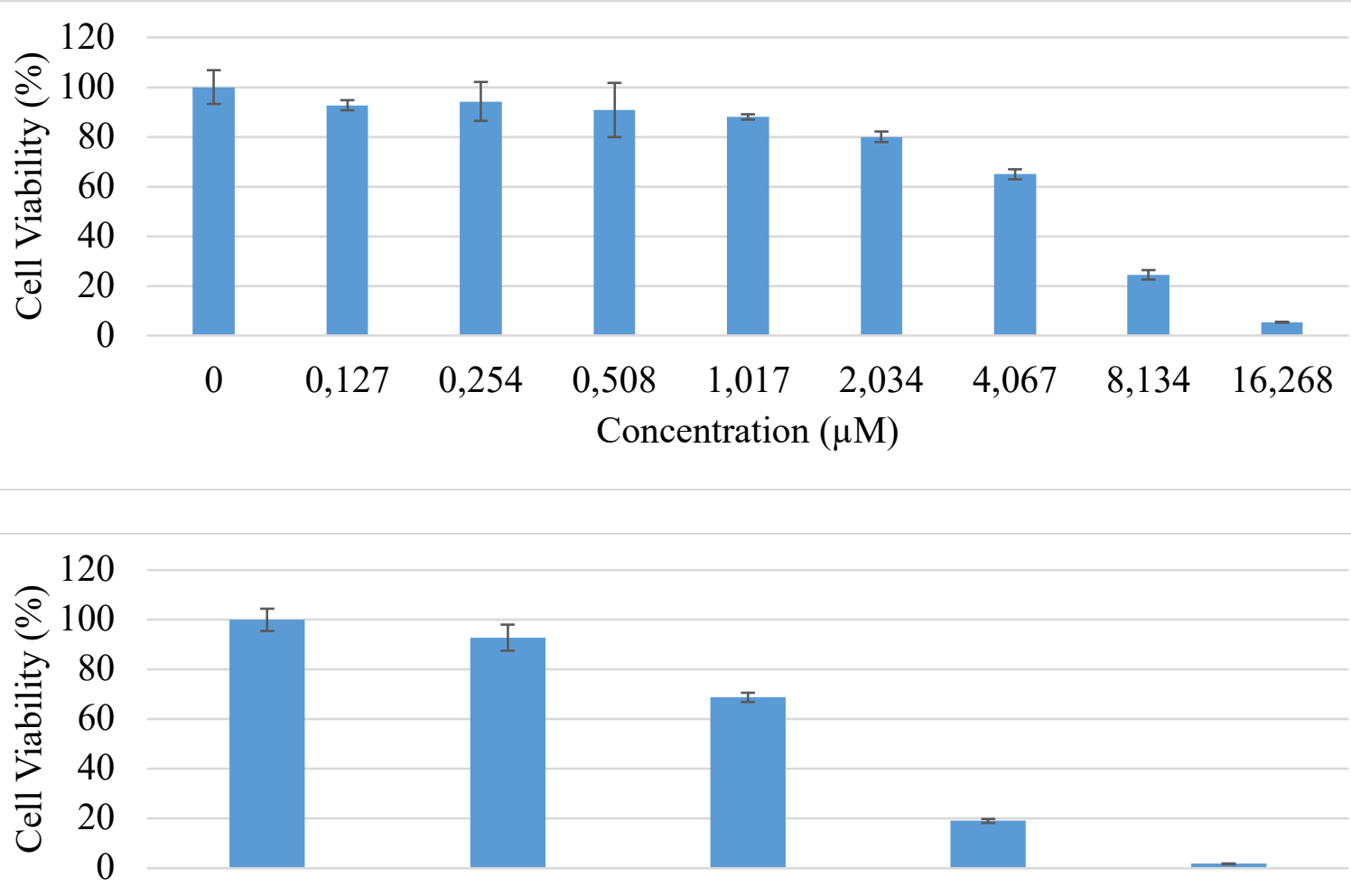

2,034

4,067

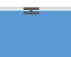

Concentration $(\mu \mathrm{M})$
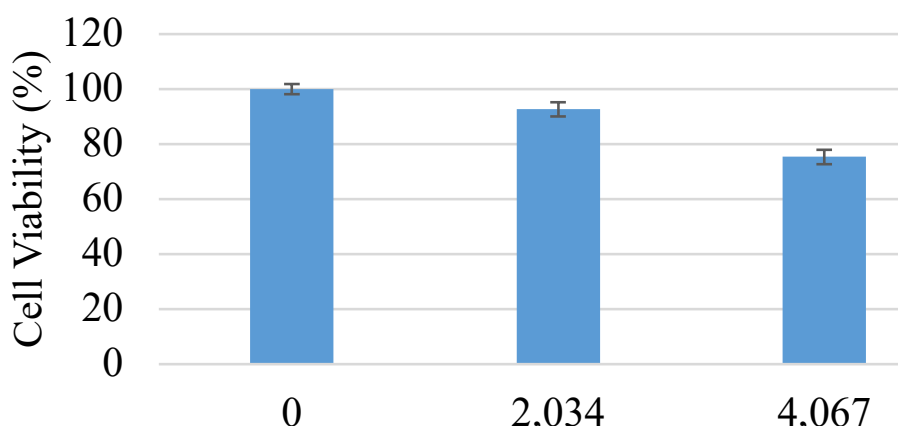

8,134

16,268

Concentration $(\mu \mathrm{M})$
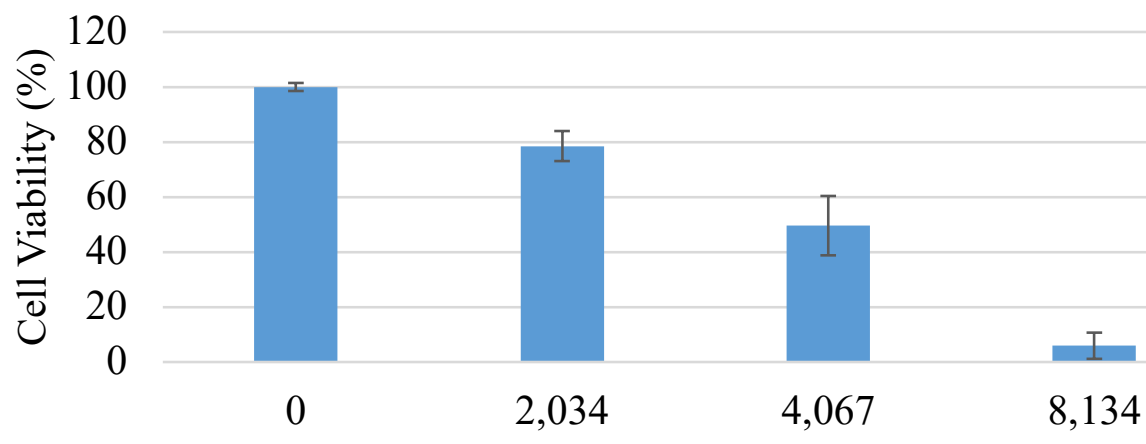

Concentration $(\mu \mathrm{M})$

Fig. S69. In vitro cytotoxicity of $\left[\mathrm{RuCl}(p\right.$-cymene $\left.)\left(\mathrm{S}_{2} \mathrm{C} \cdot \mathrm{ICy}\right)\right] \mathrm{Cl}(\mathbf{2 c})$ against $\mathrm{K} 562$ cells measured after $72 \mathrm{~h}$. 

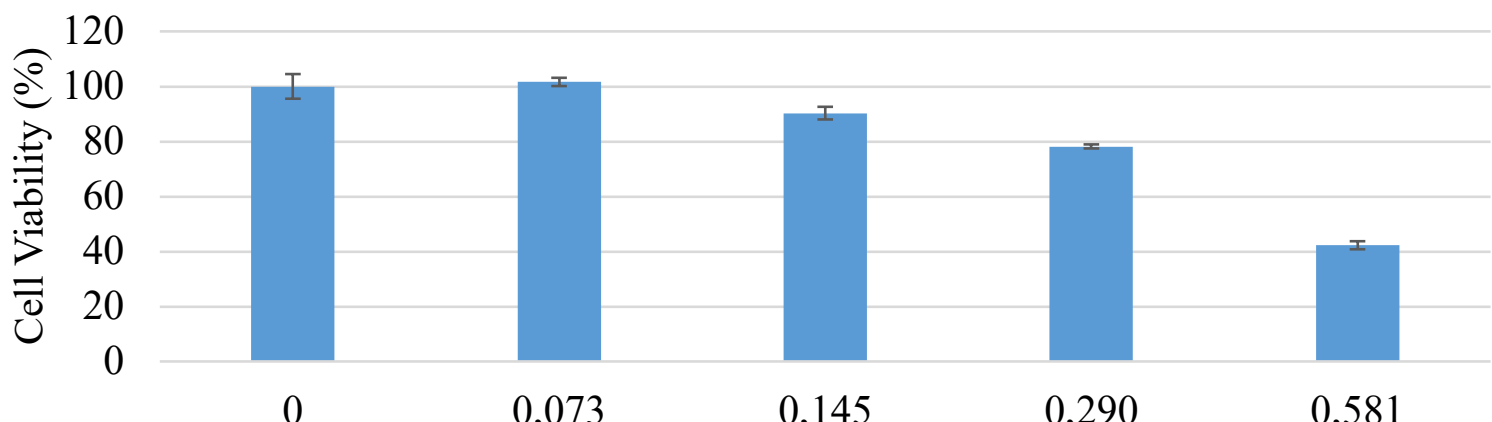

Concentration $(\mu \mathrm{M})$
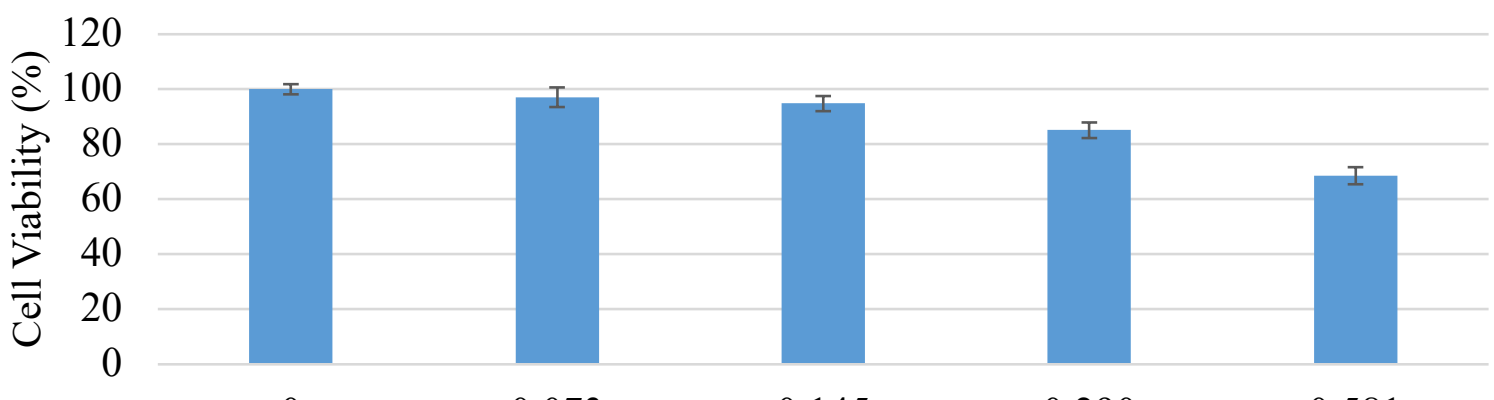

Concentration $(\mu \mathrm{M})$
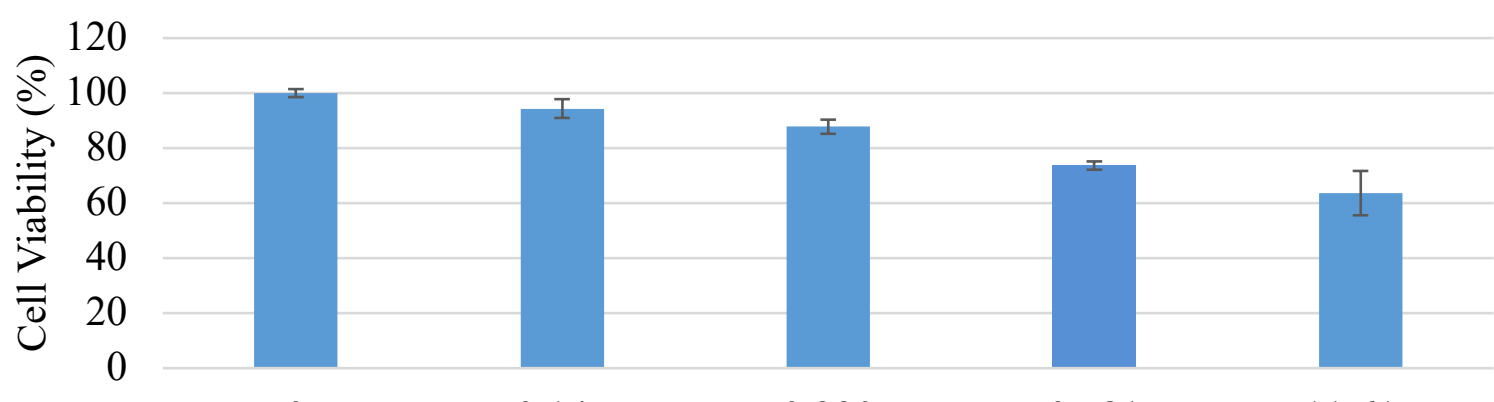

Concentration $(\mu \mathrm{M})$

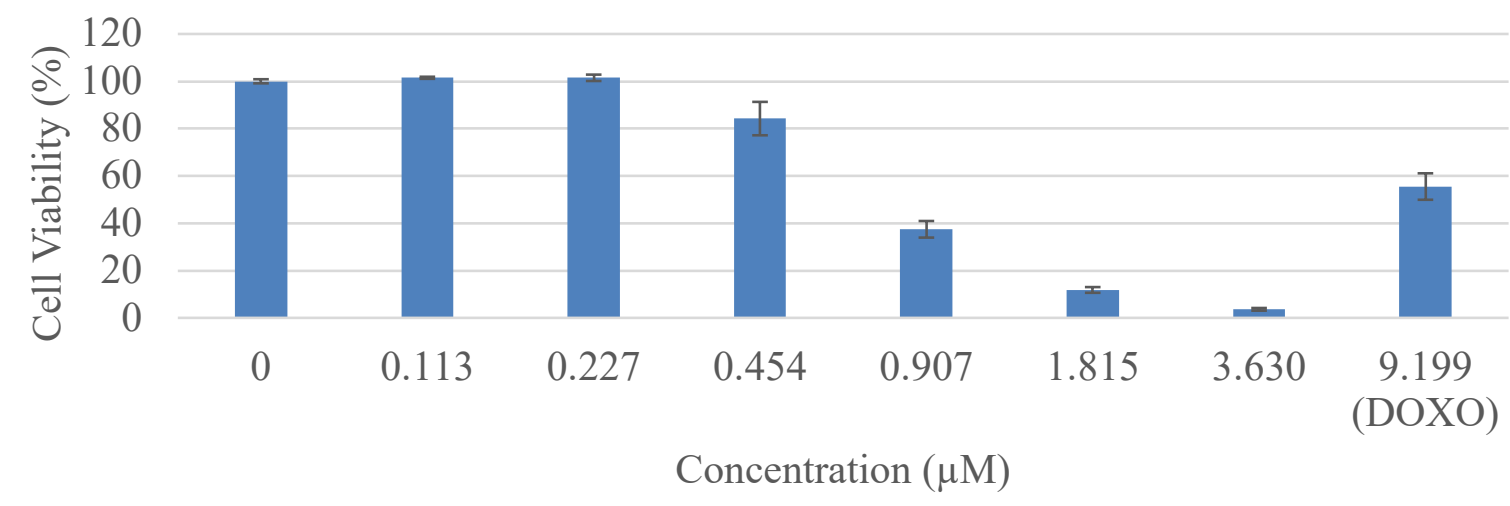

Fig. S70. In vitro cytotoxicity of $\left[\mathrm{RuCl}(p\right.$-cymene $\left.)\left(\mathrm{S}_{2} \mathrm{C} \cdot \mathrm{SIMes}\right)\right] \mathrm{Cl}(\mathbf{2 d})$ against $\mathrm{K} 562$ cells measured after $72 \mathrm{~h}$. 

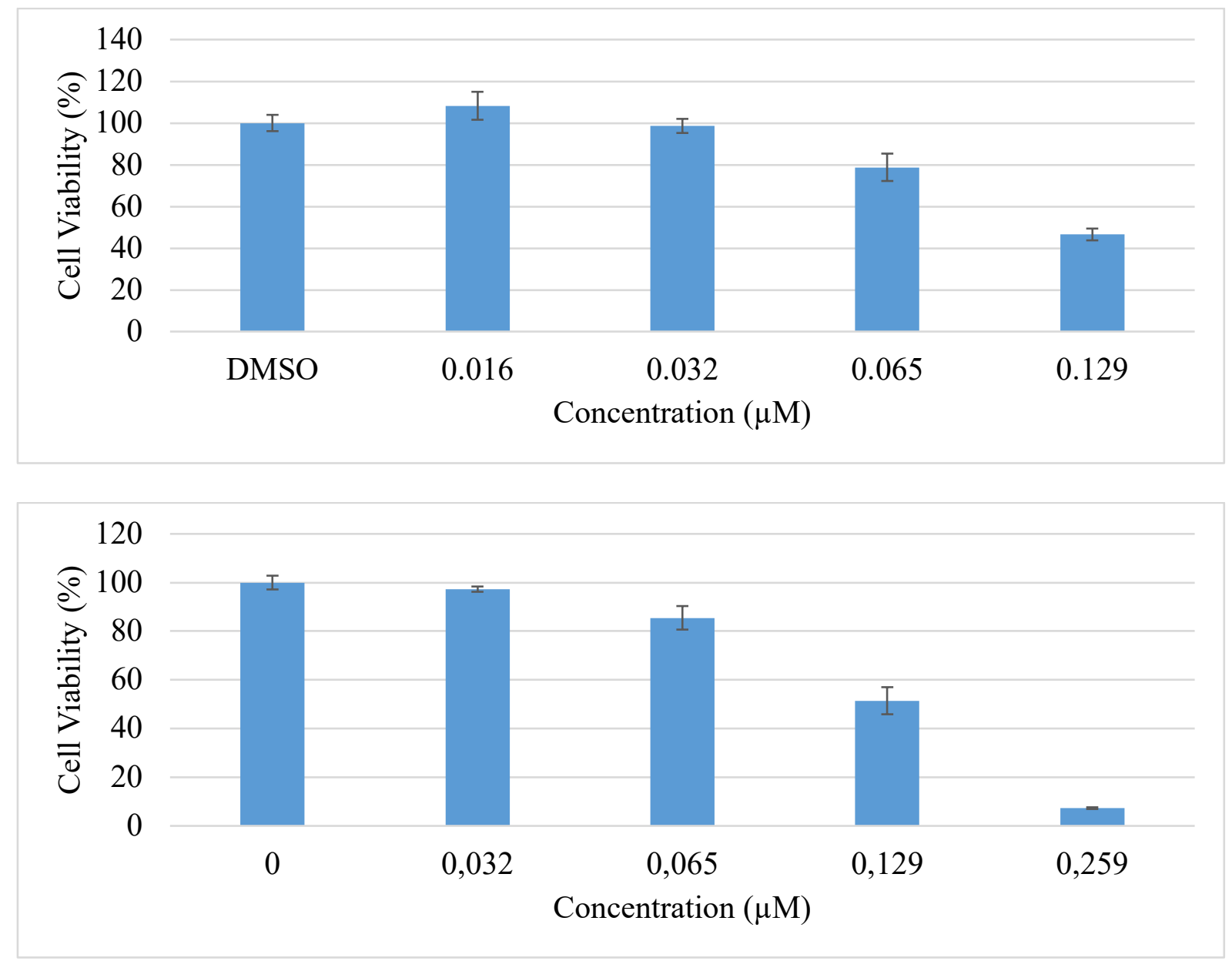

Fig. S71. In vitro cytotoxicity of $\left[\mathrm{RuCl}(p\right.$-cymene $\left.)\left(\mathrm{S}_{2} \mathrm{C} \cdot \mathrm{SIDip}\right)\right] \mathrm{Cl}(\mathbf{2 e})$ against $\mathrm{K} 562$ cells measured after $72 \mathrm{~h}$. 

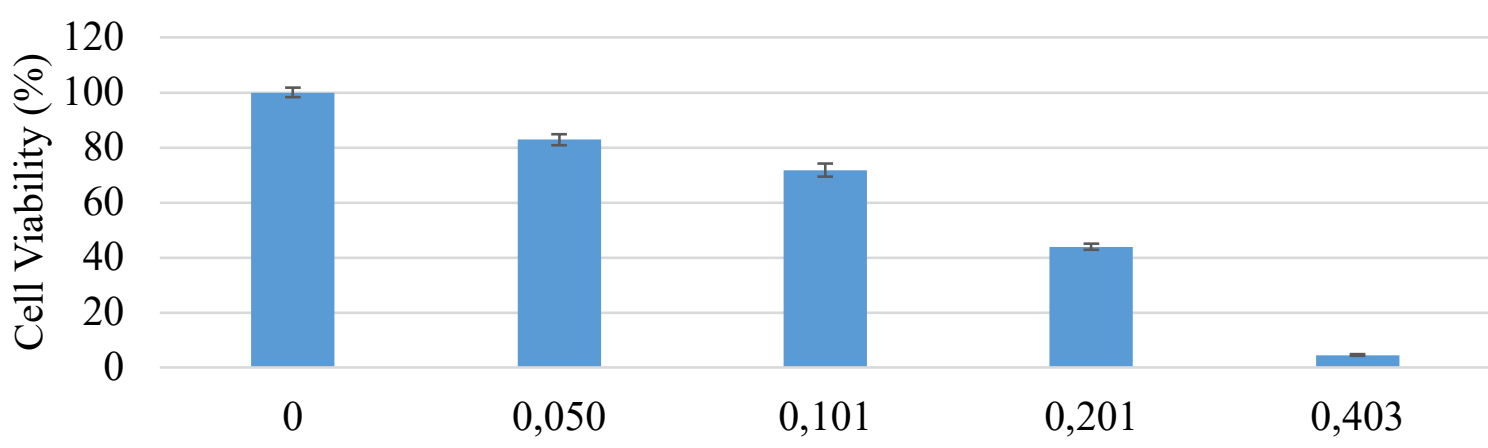

Concentration $(\mu \mathrm{M})$
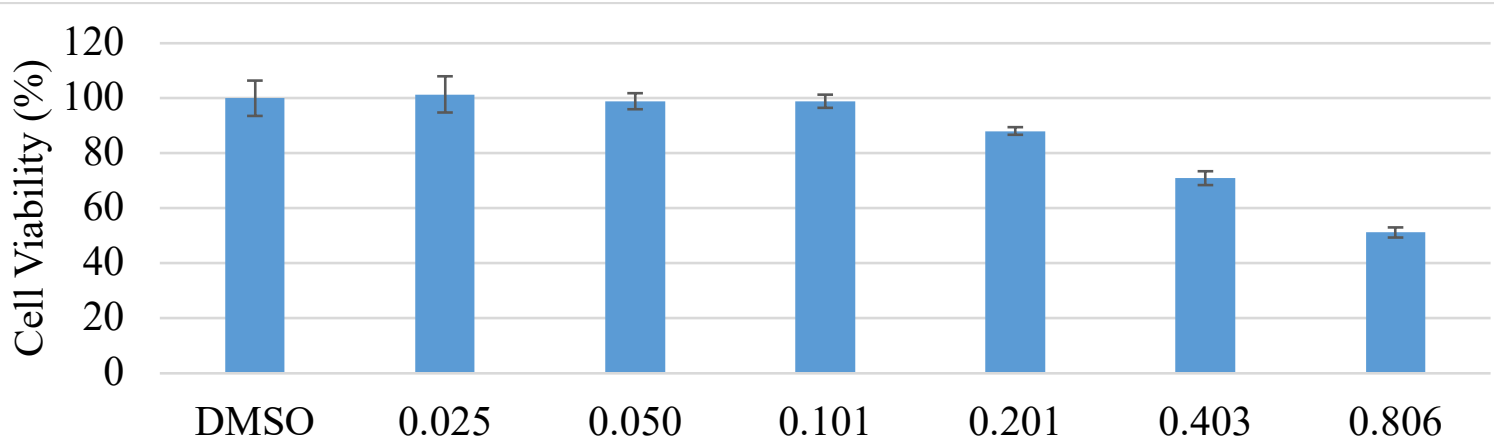

Concentration $(\mu \mathrm{M})$
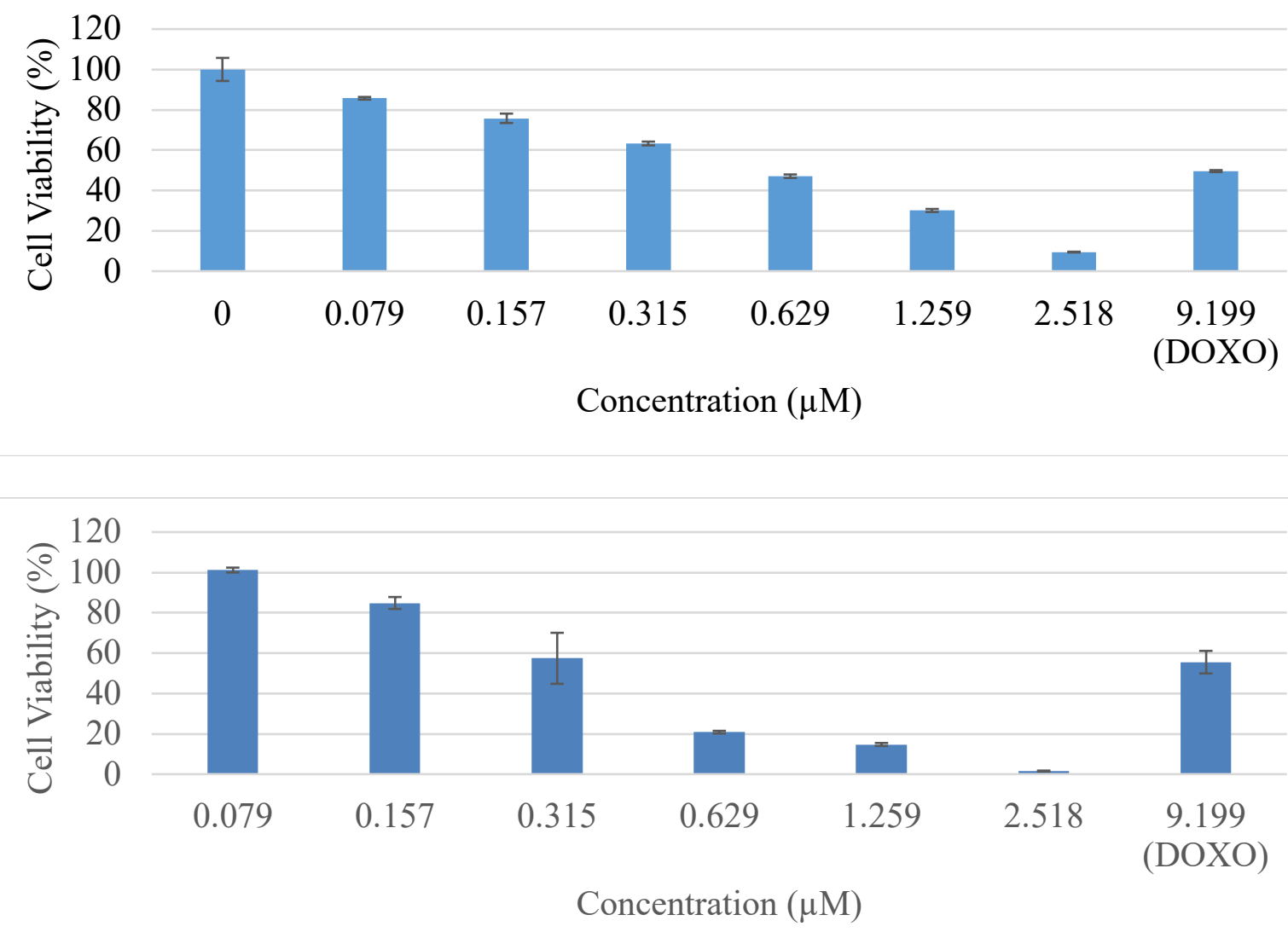

Fig. S72. In vitro cytotoxicity of $\left[\mathrm{RuCl}(p\right.$-cymene $\left.)\left(\mathrm{S}_{2} \mathrm{C} \cdot \mathrm{IMes}\right)\right]\left[\mathrm{RuCl}_{3}(\mathrm{p}\right.$-cymene $\left.)\right](\mathbf{3 a})$ against K562 cells measured after $72 \mathrm{~h}$. 

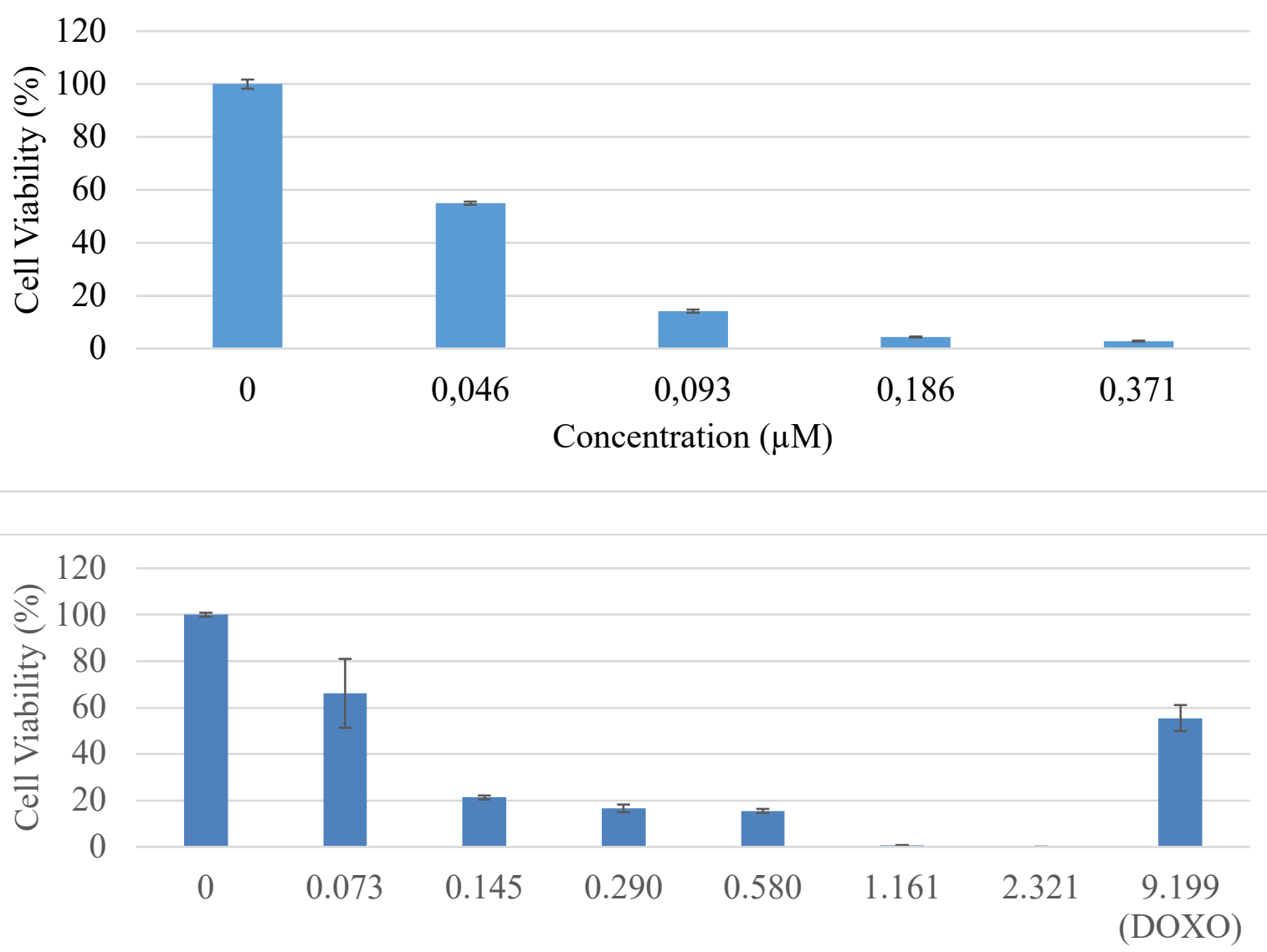

Concentration $(\mu \mathrm{M})$

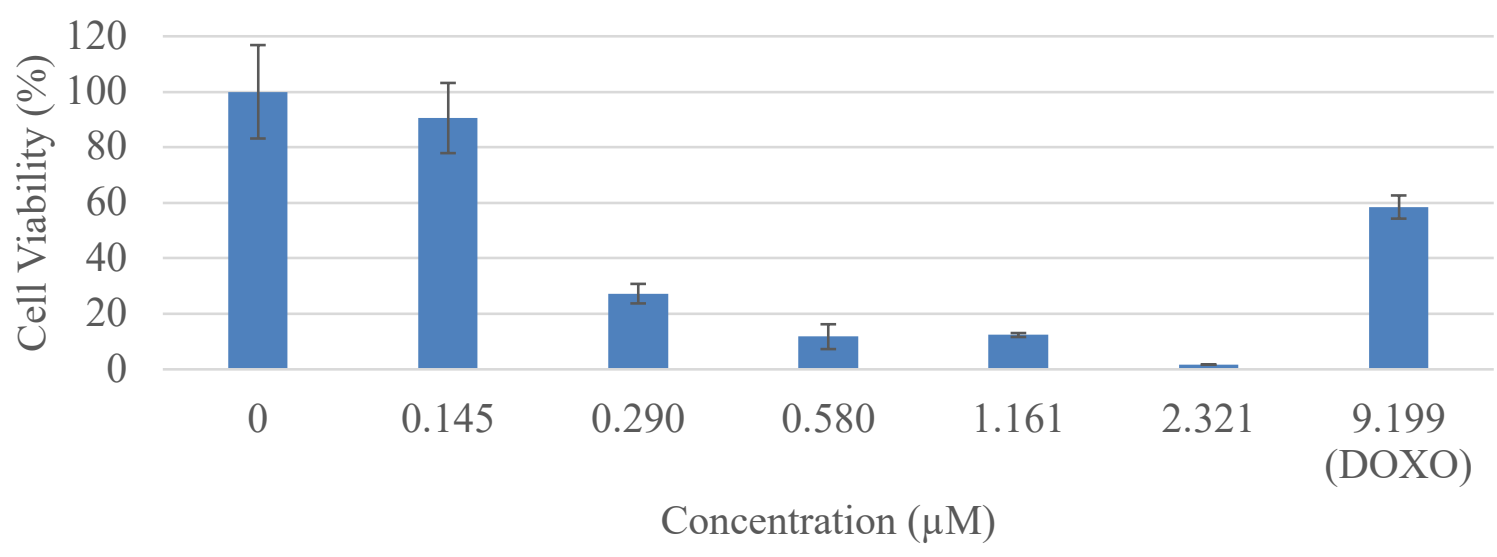

Fig. S73. In vitro cytotoxicity of $\left[\mathrm{RuCl}(p\right.$-cymene $\left.)\left(\mathrm{S}_{2} \mathrm{C} \cdot \mathrm{IDip}\right)\right]\left[\mathrm{RuCl}_{3}(\mathrm{p}\right.$-cymene $\left.)\right](\mathbf{3 b})$ against K562 cells measured after $72 \mathrm{~h}$. 


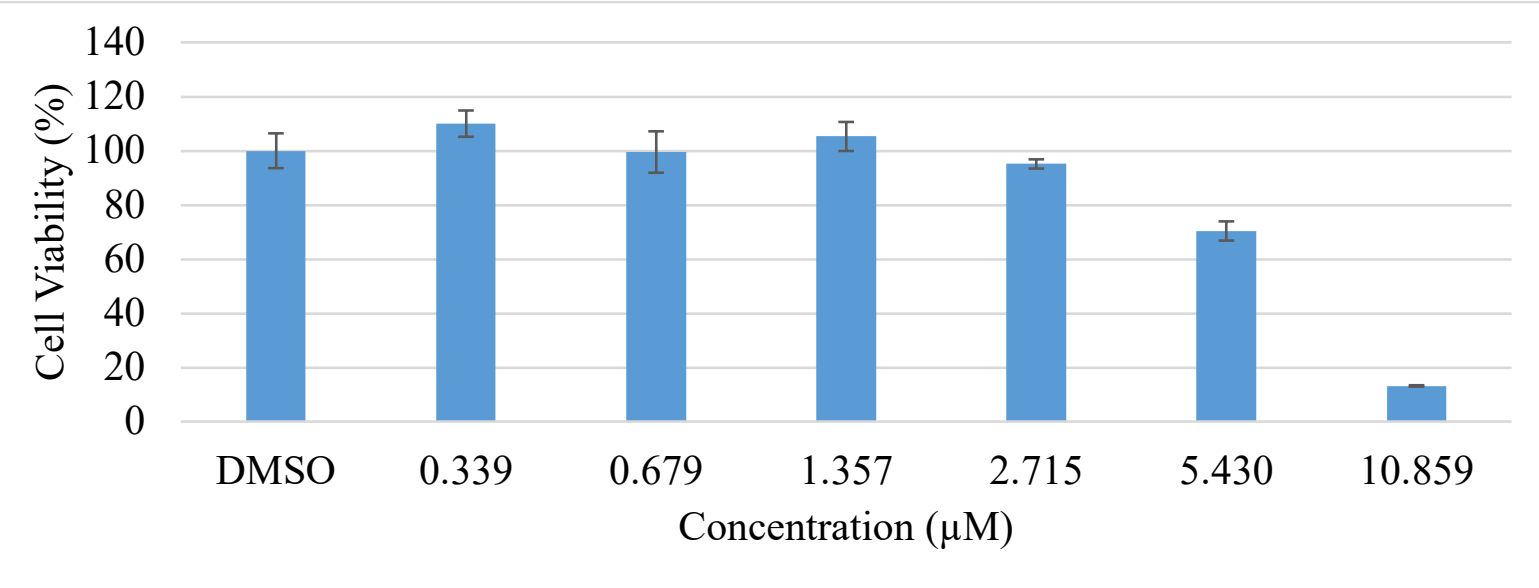

Fig. S74. In vitro cytotoxicity of $\left[\mathrm{RuCl}(p\right.$-cymene $\left.)\left(\mathrm{S}_{2} \mathrm{C} \cdot \mathrm{ICy}\right)\right]\left[\mathrm{RuCl}_{3}(\mathrm{p}\right.$-cymene $\left.)\right](3 \mathrm{c})$ against K562 cells measured after $72 \mathrm{~h}$.
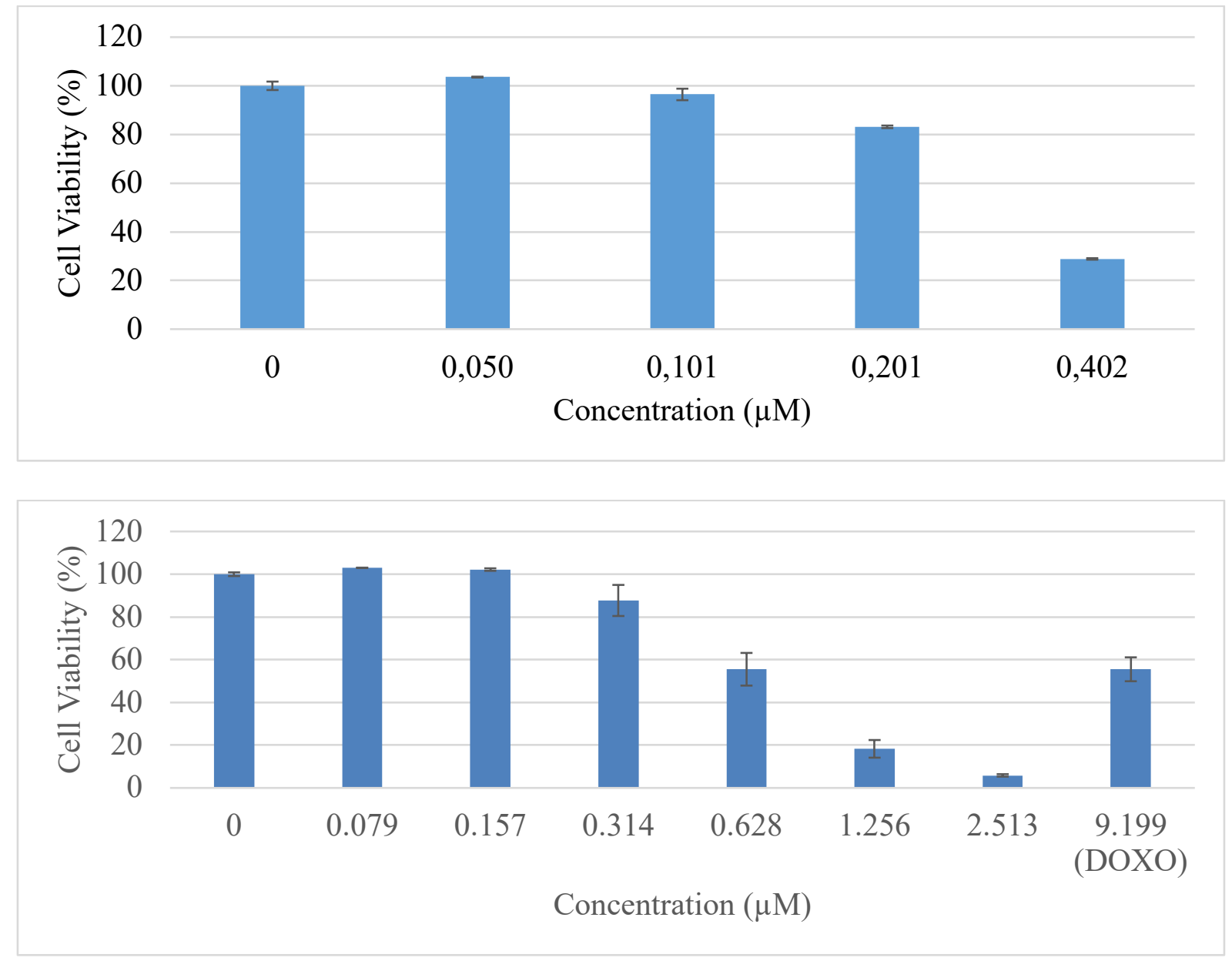

Fig. S75. In vitro cytotoxicity of $\left[\mathrm{RuCl}(p\right.$-cymene $\left.)\left(\mathrm{S}_{2} \mathrm{C} \cdot \mathrm{SIMes}\right)\right]\left[\mathrm{RuCl}_{3}(\mathrm{p}\right.$-cymene $\left.)\right](\mathbf{3 d})$ against K562 cells measured after $72 \mathrm{~h}$. 

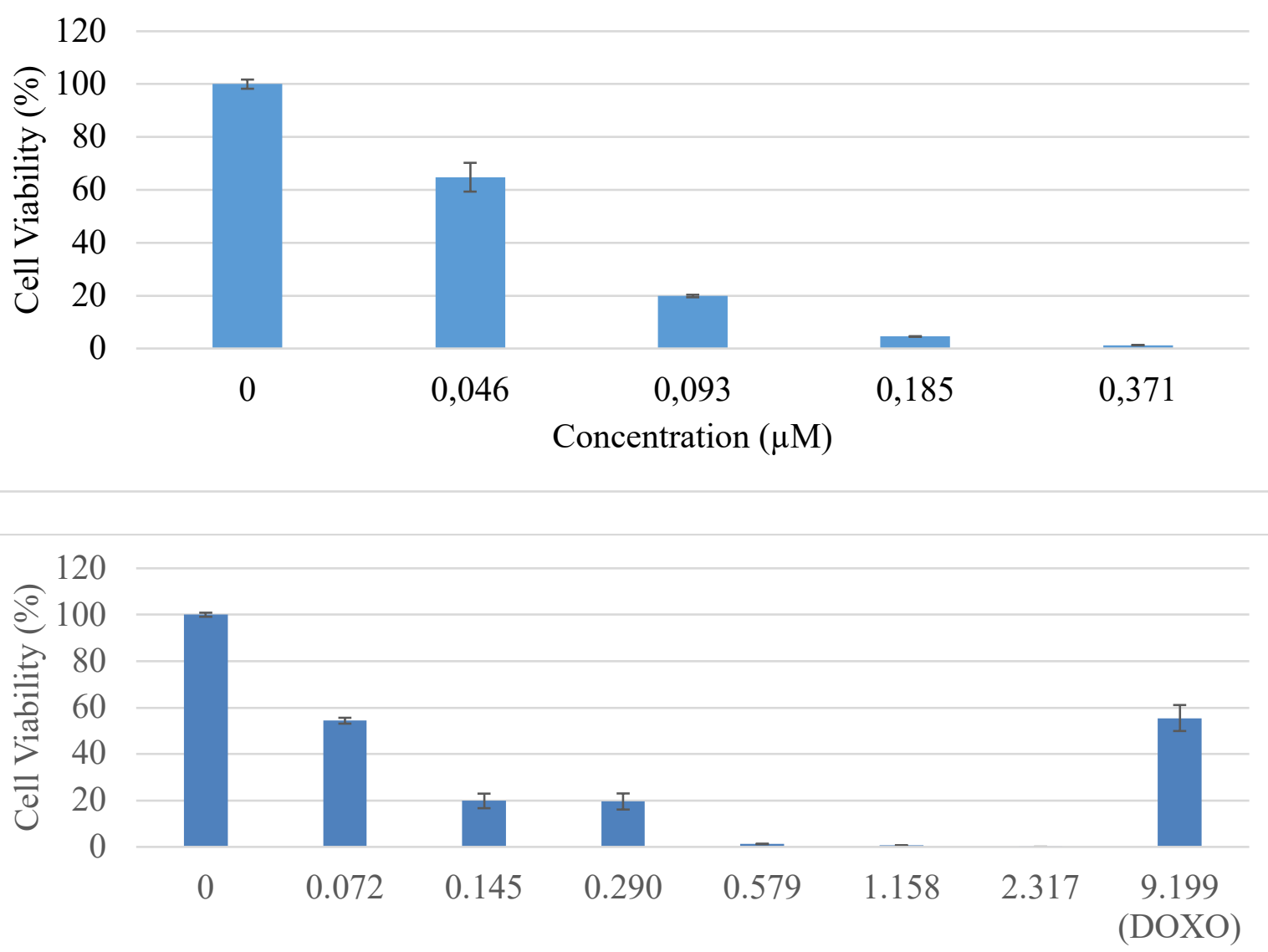

Concentration $(\mu \mathrm{M})$

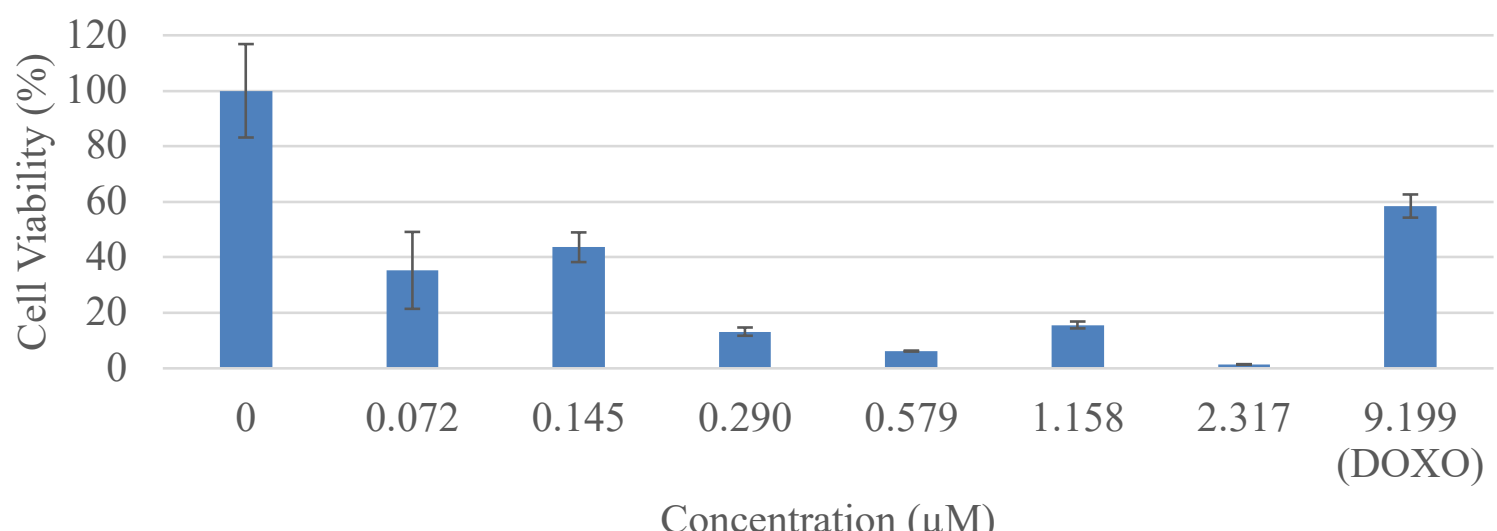

Fig. S76. In vitro cytotoxicity of $\left[\mathrm{RuCl}(p\right.$-cymene $\left.)\left(\mathrm{S}_{2} \mathrm{C} \cdot \mathrm{SIDip}\right)\right]\left[\mathrm{RuCl}_{3}(\mathrm{p}\right.$-cymene $\left.)\right](3 \mathbf{e})$ against K562 cells measured after $72 \mathrm{~h}$. 


\section{References}

(1) Delaude, L.; Demonceau, A.; Wouters, J. Assessing the Potentials of Zwitterionic NHC $\cdot \mathrm{CS}_{2}$ Adducts for Probing the Stereoelectronic Parameters of N-Heterocyclic Carbenes. Eur. J. Inorg. Chem. 2009, 1882-1891.

(2) Bruker APEX II, Bruker AXS Inc.; Madison, WI, USA, 2004.

(3) Clark, R. C.; Reid, J. S. The Analytical Calculation of Absorption in Multifaceted Crystals. Acta Crystallogr., Sect. A: Fundam. Crystallogr. 1995, 51, 887-897.

(4) Burla, M. C.; Caliandro, R.; Camalli, M.; Carrozzini, B.; Cascarano, G. L.; De Caro, L.; Giacovazzo, C.; Polidori, G.; Spagna, R. SIR2004: An Improved Tool for Crystal Structure Determination and Refinement. J. Appl. Crystallogr. 2005, 38, 381-388.

(5) Sheldrick, G. M. SHELX-97 (SHELXS 97 and SHELXL 97), Programs for Crystal Structure Analyses; University of Göttingen, Göttingen (Germany), 1998.

(6) Sheldrick, G. M. SADABS, Programs for Scaling and Correction of Area Detection Data; University of Göttingen, Göttingen (Germany), 1996.

(7) Tomani, J. C. D.; Gainkam, L. O. T.; Nshutiyayesu, S.; Mukazayire, M. J.; Ribeiro, S. O.; Stevigny, C.; Frederich, M.; Muganga, R.; Souopgui, J. An ethnobotanical survey and inhibitory effects on NLRP3 inflammasomes/Caspase-1 of herbal recipes' extracts traditionally used in Rwanda for asthma treatment. J. Ethnopharmacol. 2018, 227, 29-40.

(8) Tomani, J. C. D.; Kagisha, V.; Tchinda, A. T.; Jansen, O.; Ledoux, A.; Vanhamme, L.; Frederich, M.; Muganga, R.; Souopgui, J. The inhibition of NLRP3 inflammasome and IL-6 production by hibiscus noldeae baker $\mathrm{f}$. derived constituents provides a link to its antiinflammatory therapeutic potentials. Molecules 2020, 25, art. no. 4693. 